\title{
Investigation of Plasma/Ion Vapor Deposition (IVD) Hybrid Coatings for Corrosion Protection and Plasma Destruction of Oral Bacteria
}

\author{
A Dissertation \\ Presented to \\ The Faculty of the Graduate School \\ University of Missouri \\ In Partial Fulfillment \\ of the Requirements for the Degree \\ Doctor of Philosophy \\ by \\ Adam Blumhagen \\ Dr. Qingsong Yu, Dissertation Adviser \\ July 2013
}


The undersigned, appointed by the Dean of the Graduate School, have examined the dissertation entitled

\section{Investigation of Plasma/Ion Vapor Deposition (IVD) Hybrid Coatings for Corrosion Protection and Plasma Destruction of Oral Bacteria}

presented by

\section{Adam Blumhagen}

a candidate for the degree of

\section{Doctor of Philosophy}

and hereby certify that in their opinion it is worthy of acceptance.

Dr. Qingsong Yu

Dr. Hao Li

Dr. Stephen J. Lombardo

Dr. Azlin Mustapha

Dr. Mengshi Lin

Dr. Meng Chen 


\section{ACKNOWLEDGEMENTS}

I want to acknowledge my advisor Dr. Yu for taking me on as a graduate student and helping me to succeed. His support and critiques have made me a better research professional and a better person.

I would like to thank The Boeing Company, particularly Bruce Griffin, for managing the corrosion and hydrogen embrittlement work, the United States Air Force for funding that research, Mike Bridger for help with IVD setup, the University of Missouri's EMC core, Missouri University of Science and Technology Material's Research Center, and Dr. Anthony Caruso's group at the University of Missouri-Kansas City for XPS analysis.

Thank you to my dissertation committee, Dr. Meng Chen, Dr. Hao Li, Dr. Stephen Lombardo, Dr. Azlin Mustapha, and Dr. Mengshi Lin. A special thanks to Dr. Mustapha for guidance and the use of her lab.

Thanks to my colleagues: Andrew Ritts, John Jones, Qing Hong, Xiaqing Dong, Prashant, and Young Jo Kim. Your help and encouragement was priceless.

Thank you to my parents for supporting me throughout my entire life but most specifically during the past years in college. Your guidance has kept me on the path to achieving all that I have. 


\section{TABLE OF CONTENTS}

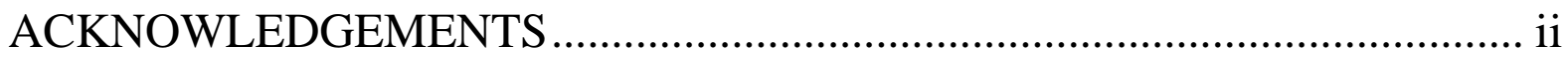

TABLE OF FIGURES ................................................................................ vii

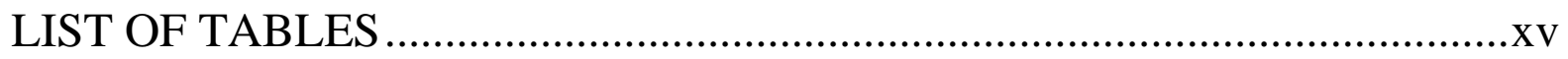

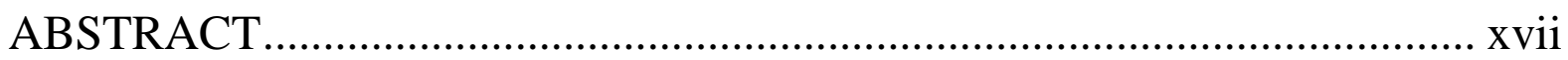

Chapter 1 General Introduction .................................................................... 1

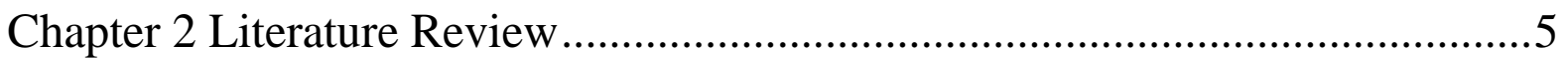

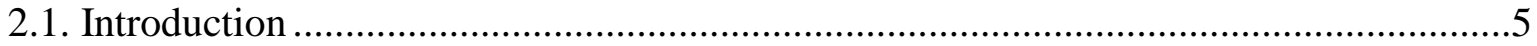

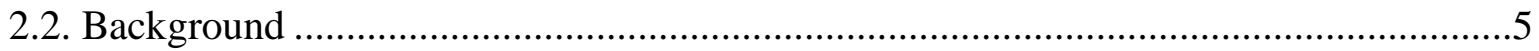

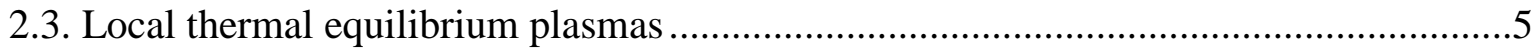

2.4. Non-Local Thermal Equilibrium Plasmas..................................................................

2.4.1. Non-LTE plasma coating ………………………............................................10

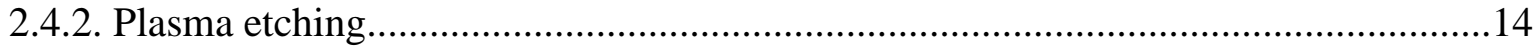

2.4.3. Plasmas in the medical field ...........................................................................15

2.4.4. Plasma modification of surfaces ....................................................................17

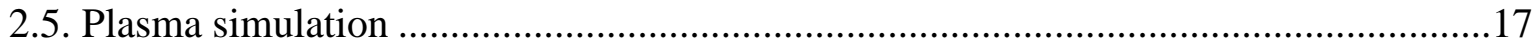

2.6. Plasma cost benefits ......................................................................................18

2.7. Corrosion and Hydrogen Re-embrittlement ............................................................18

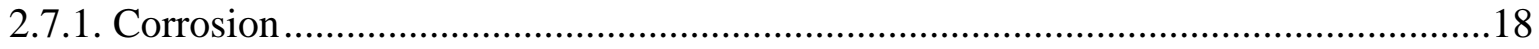

2.8. Hydrogen Re-embritllement

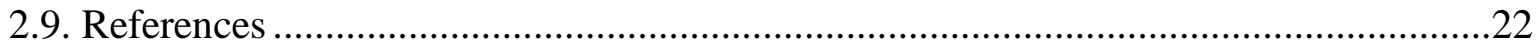

Chapter 3 Hydrogen Re-Embrittlement Protection of IVD Coated Steel by

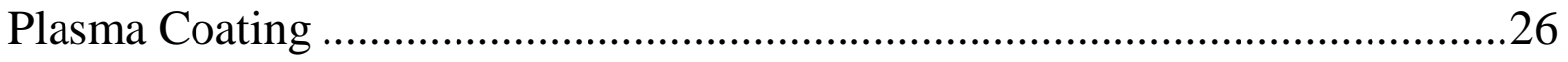

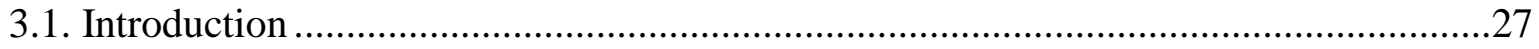

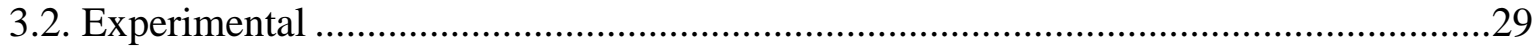

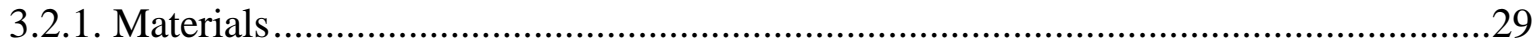

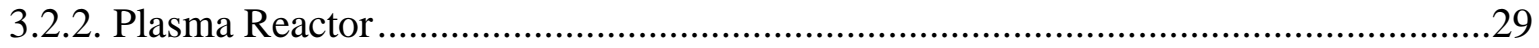

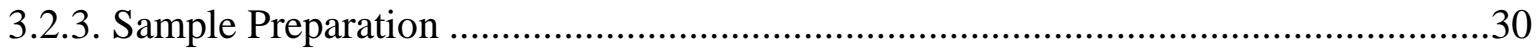

3.2.4. Hydrogen Embrittlement ...................................................................................

3.2.5. Hydrogen Re-embrittlement ...............................................................................

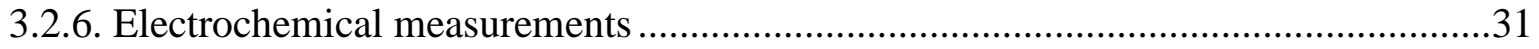

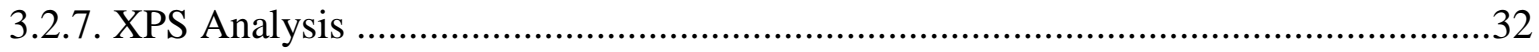




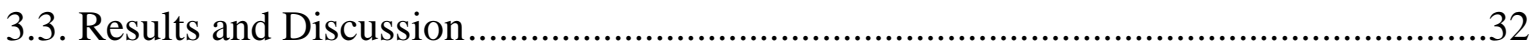

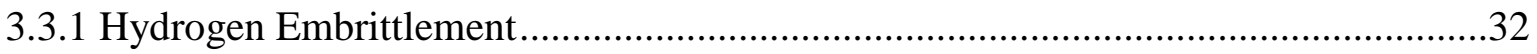

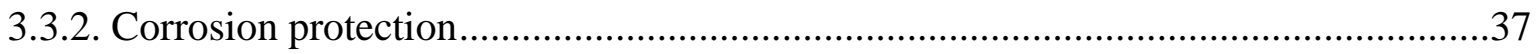

3.3.3. Plasma nanocoating characterization ………………............................................40

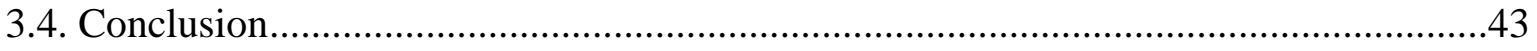

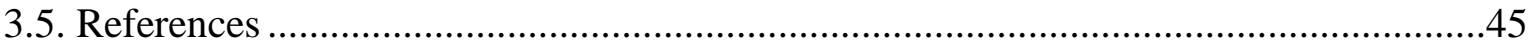

Chapter 4 Construction of an Ion Vapor Deposition Plasma Nanocoating

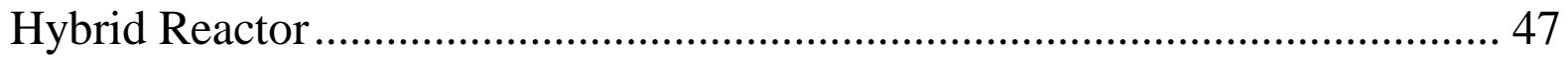

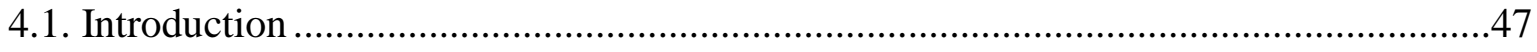

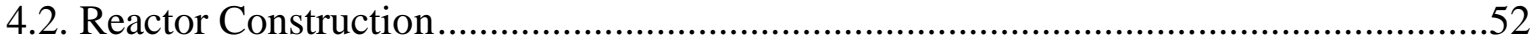

4.2.1. Construction of the Vacuum System.....................................................................52

4.2.2. Modification of Boat Clamps ...........................................................................54

4.2.3. Shutter Design and Construction ……………................................................56

4.2.4. Wire Feeder Modification ............................................................................57

4.2.5. Installation of Pressure Controller, Baratrons, and Mass Flow Controllers................57

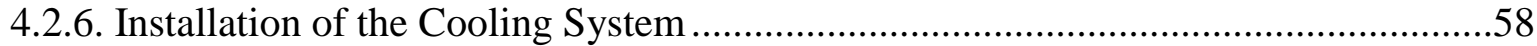

4.2.7 Installation of the Heating Boat Power Supply ………..........................................58

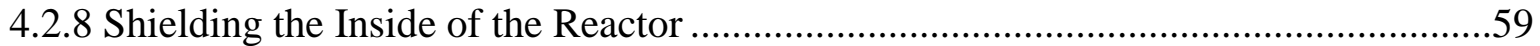

4.2.9 IVD Operating Procedure

4.2.10. Troubleshooting Plasma Quenching During IVD ...................................................60

4.2.11. Attempts at Achieving 100\% IVD Through Modification of Al Feed Rates...........65

4.2.12. Determination of Cooling Parameters for Achieving 100\% IVD ............................69

4.3. Production and Repeatability of a Class II IVD Al Coating ..........................................73

4.4. Application of Plasma Nanocoating .......................................................................76

4.5. Evaluation of Possible Contamination of IVD from Plasma Coating..........................79

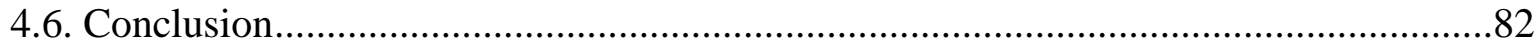

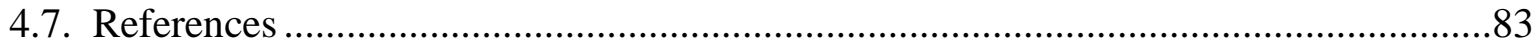

Chapter 5 Corrosion Protective Coatings Produced with a Hybrid IVD-Plasma

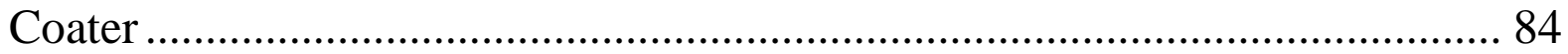

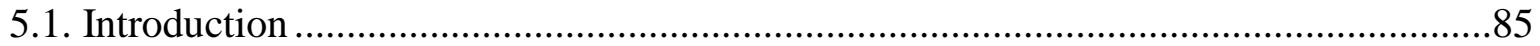

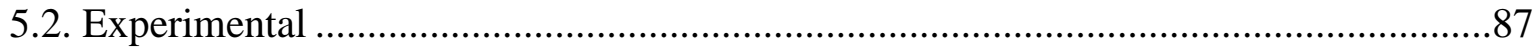

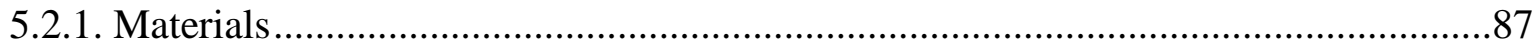

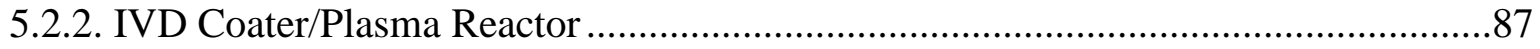

5.2.3. Corrosion Testing Samples Preparation .......................................................................89 


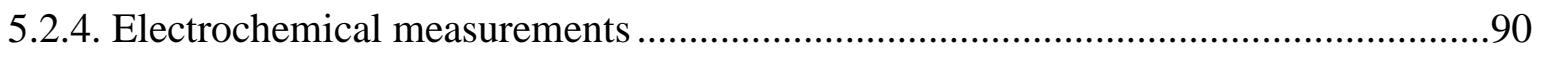

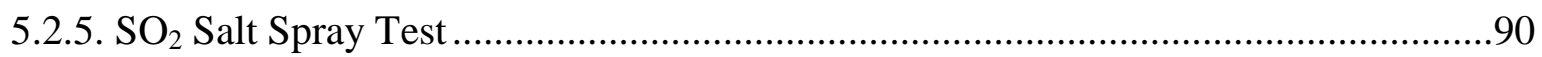

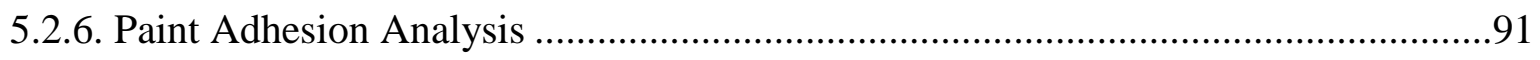

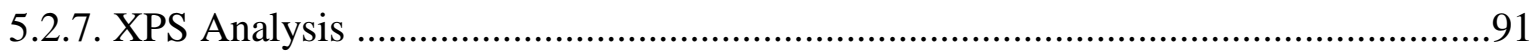

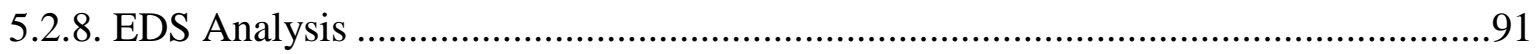

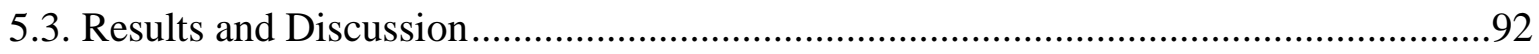

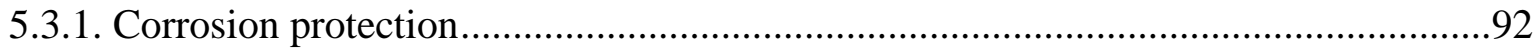

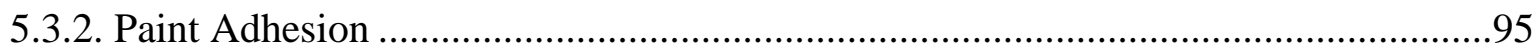

5.3.3. Evaluation of Possible Contamination ……………….......................................95

5.3.4. Plasma Nanocoating Characterization ………………......................................97

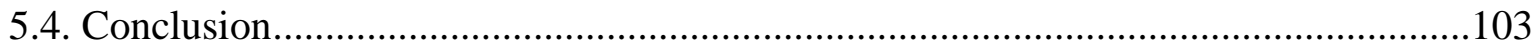

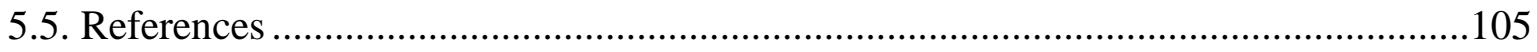

Chapter 6 Plasma Sterilization of Oral Bacteria............................................ 107

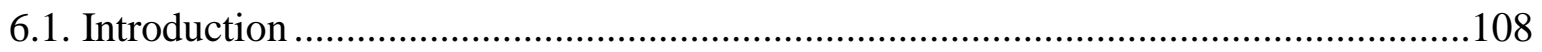

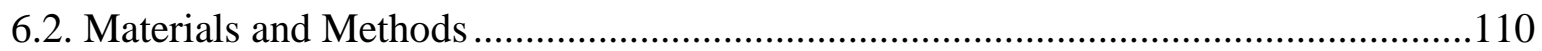

6.2.1. Materials and Bacterium preparation ...................................................................110

6.2.2. Plasma treatment and brush construction............................................................111

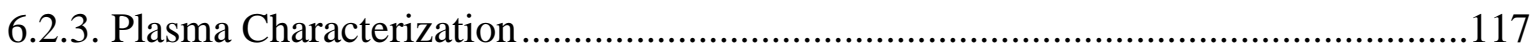

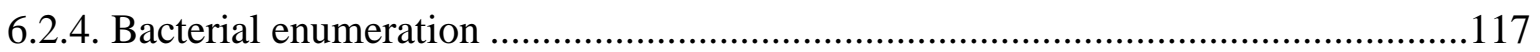

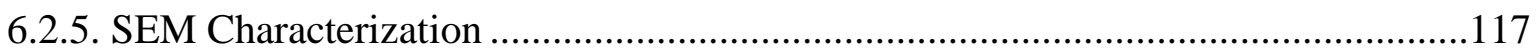

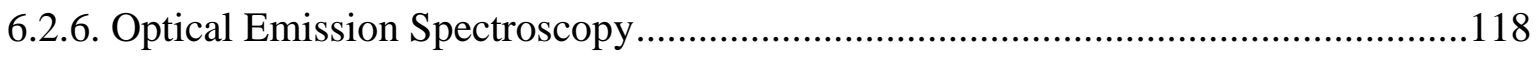

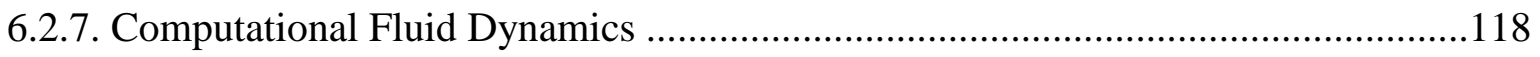

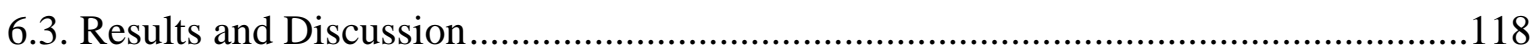

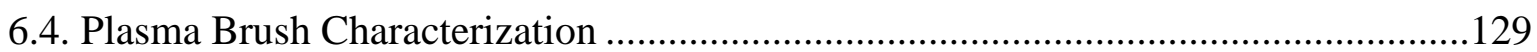

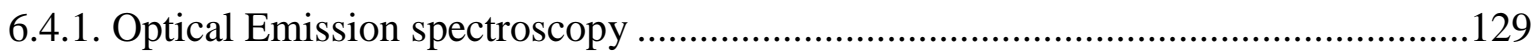

6.4.2. Optical Emission Spectroscopy Performed During Plasma Sterilization .................133

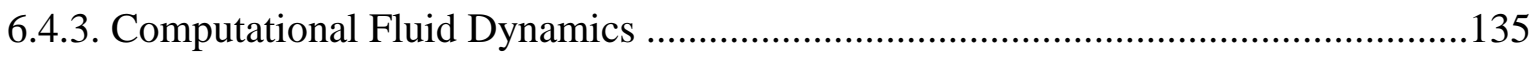

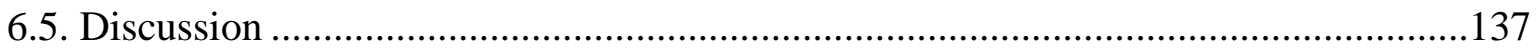

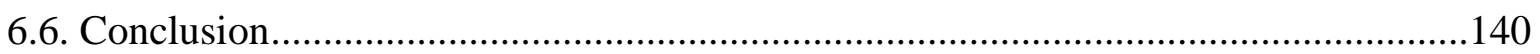

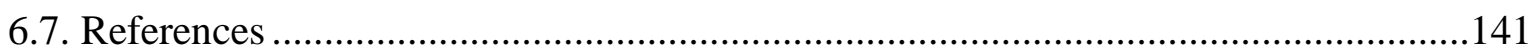

Chapter 7 Disinfection of Oral Bacteria on Dentin by Atmospheric Pressure

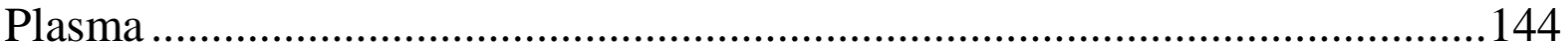

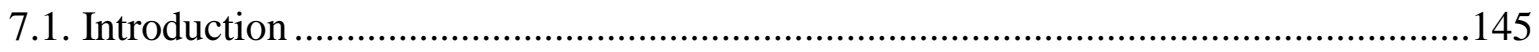

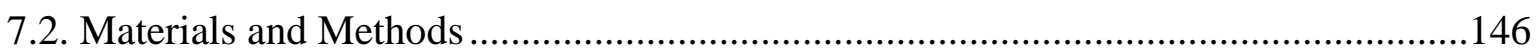




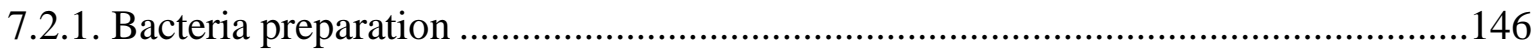

7.2.2. Treatment of bacteria seeded on microchannel walls ..............................................147

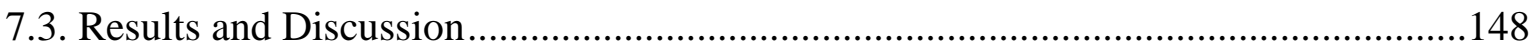

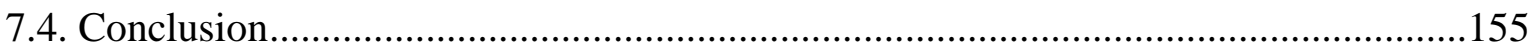

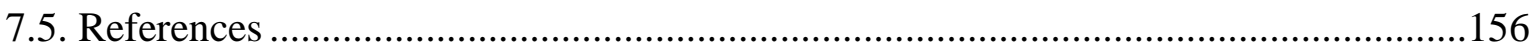

Chapter 8 Conclusions and Future Prospects ............................................... 158

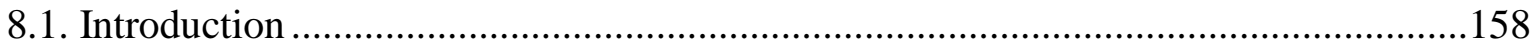

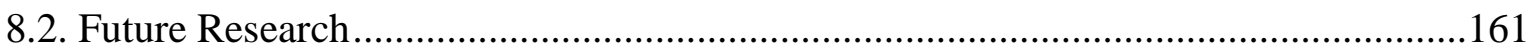

8.2.1 Plasma coatings for corrosion and hydrogen re-embrittlement resistance.................161

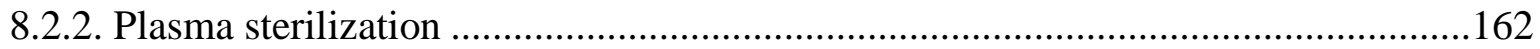

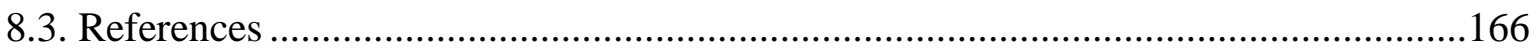

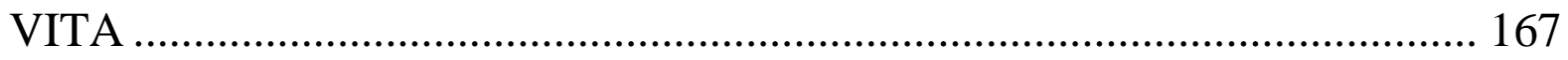




\section{TABLE OF FIGURES}

Figure 3.1. SEM Images of ASTM F 519 1a.1 Bar of specimen \#18 shown in Table 3.3: a) Load Bar End; b) Origin of Failure; c) Increased Magnification of Origin; d) Overload and

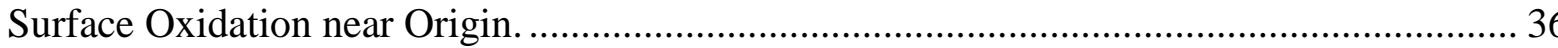

Figure 3.2. Typical anodic potentiodynamic polarization curves of TMS plasma coated and bare IVD Al coated steel specimens. 38

Figure 3.3. XPS scan spectra taken from the top surface of TMS plasma coated and bare IVD Al coated steels.

Figure 3.4. High resolution XPS spectra O1S peak taken from the top surface of TMS

plasma nanocoating 42

Figure 3.5. High resolution XPS spectra C1S peak taken from the top surface of TMS

plasma nanocoating 42

Figure 3.6. High resolution XPS spectra Si2P peak taken from the top surface of TMS

plasma nanocoating. 43

Figure 4.1. SEM of physical vapor deposited aluminum 52

Figure 4.2. A depiction of the IVD/Plasma reactor vacuum system. 53

Figure 4.3. Photo of the assembled vacuum system. 54

Figure 4.4. 3D depictions of the reactor chamber and boat holder with the proposed boat clamps in red and the estimated boat location in blue; two options for the clamp design...... 55 Figure 4.5. A $2 \mathrm{D}$ depiction of the final boat clamp design (blue) shown in relation to the reactor; dimensions are inches. 55

Figure 4.6. Photo of new boat clamps for the larger reactor 55 
Figure 4.7. A depiction of the basic shutter design (left), a photo of the constructed shutter (right). 56

Figure 4.8. Photo of wire feeder and heating boat..................................................... 57

Figure 4.9. Photo of larger lugs for powering the heating boat..................................... 59

Figure 4.10. Photo of heating of the heating boat.......................................................... 59

Figure 4.11. A depiction of the electrode setup where the wire holding the panel up sets on a glass tube that is insulated from the rod; IVD cannot penetrate into the glass tube.

Figure 4.12. A depiction of the electrode setup where two glass tubes insulate the wires holding the panel up

Figure 4.13. A depiction of the electrode setup where the panel is held up by a fiber glass string and IVD is not allowed to coat the string because of a shielding panel. 62

Figure 4.14. A depiction of the electrode setup; the panel hangs from the cathode; the cathode is surrounded by a glass tube so IVD cannot coat the Teflon insulation.

Figure 4.15. SEM pictures of steel panels coated with aluminum from trials where plasma stayed on during evaporation of the aluminum 64

Figure 4.16. Photo of the substrate holder and electrode setup. Teflon spacers located deep within glass tubes keep IVD from coating insulators.

Figure 4.17. SEM image of a panel coated for $4 \mathrm{~min} \sim 5 \mathrm{in} / \mathrm{min}$ 66

Figure 4.18. SEM image of a panel coated for $2 \mathrm{~min} \sim 8.75 \mathrm{in} / \mathrm{min}$.

Figure 4.19. SEM image of a panel coated for $3 \mathrm{~min} 30 \mathrm{sec} \sim 12 \mathrm{in} / \mathrm{min}$. 67

Figure 4.20. SEM image of a panel coated for $4 \mathrm{~min} \sim 17 \mathrm{in} / \mathrm{min}$. 68

Figure 4.21. SEM image of a panel coated for $8 \mathrm{~min} @ 4 \mathrm{in} / \mathrm{min}(3.2-4.5 \mu \mathrm{m})$. 68 
Figure 4.22. SEM image of a panel coated for $3 \mathrm{~min} 10 \mathrm{sec}$ Ramped from $\sim 4 \mathrm{in} / \mathrm{min}$ to 18 in/min over $\sim 30 \sec (3 / 23 / 10)$

Figure 4.23. SEM image of a panel coated at $2 \mathrm{~min} @ 4 \mathrm{in} / \mathrm{min}, 2 \mathrm{~min} @ 8 \mathrm{in} / \mathrm{min}(1-3 \mu \mathrm{m})$.

Figure 4.24. SEM image of a panel coated for 2 min @ 4 in/min, 2 min @ 8 in $/ \mathrm{min}$ cycled twice with 30 minutes between cycles $(3.3-5.5 \mu \mathrm{m})$.

Figure 4.25. SEM image of a panel coated for 2 min @ 4 in/min, 2 min @ 8 in $/ \mathrm{min}$ cycled twice with no time between cycles $(3.7-5.4 \mu \mathrm{m})$.

Figure 4.26. SEM image of a panel coated for $2 \mathrm{~min} @ 4 \mathrm{in} / \mathrm{min}, 2 \mathrm{~min} @ 8 \mathrm{in} / \mathrm{min}$ cycled five times with 30 minutes between cycles $(8.8-11.9 \mu \mathrm{m})$.

Figure 4.27. SEM image of a panel coated at $5 \mathrm{in} / \mathrm{min}$ for 2 minutes and $8 \mathrm{in} / \mathrm{min}$ for 2 minutes for five cycles.

Figure 4.28. Locations of measurements for test panels 75

Figure 4.29. SEM image of IVD Al coated steel, from seen from the top (left) and from the cross section (right) 76

Figure 4.30. Deposition time dependence of plasma coating thickness and refractive indices. Plasma conditions were: $1 \mathrm{sccm}$ argon, $1 \mathrm{sccm}$ TMS, 50 mTorr system pressure, - $900 \mathrm{~V}$ DC potential. 77

Figure 4.31. Plasma coating uniformity on a $4 \times 6$ inch steel panel in vertical (6 inch direction) and horizontal (4 inch direction) positions. Plasma conditions were: $1 \mathrm{sccm}$ argon, $1 \mathrm{sccm}$ TMS, 50 mTorr system pressure, - 900 V DC potential................................................ 77

Figure 4.32. IVD coated panel with subsequent plasma coating................................... 78 
Figure 4.33. Dependence of plasma coatings on specimen size. Plasma conditions were: 1 sccm argon, 1 sccm TMS, 50 mTorr system pressure, - 900 V DC potential. ..................... 79

Figure 4.34. Images of panel holder electrodes after plasma coating............................... 80

Figure 4.35. Images of electrode/panel holders for IVD Al and TMS plasma coatings after IVD Al and TMS plasma coating.

Figure 4.36. Images of electrode/panel holders for IVD Al and TMS plasma coatings after 2:1 oxygen to argon plasma cleaning for 10 minutes at 50 mTorr.

Figure 5.1. Depiction of the IVD-plasma coating hybrid reactor

Figure. 5.2. SEM image of IVD Al coated steel, from seen from the top (left) and from the cross section (right)

Figure 5.3. Typical anodic polarization curves of IVD Al coated steel with and without various plasma coating. 93

Figure 5.4. Typical images of IVD coated panels after neutral salt fog testing; a) with chromate conversion coating after 504 hours, b) with TMS plasma coating after 504; c) Boeing produced IVD with chromate conversion coating after 504 hours; d) without further coating after 336 hours. 94

Figure 5.5. SEM images of IVD coated panels; produce by Boeing (left) and hybrid reactor (right). 95

Figure 5.6. Typical image of IVD coated panel with coating applied by hybrid coater following completion of all plasma coating trials, Corrosion Resistance Test-504 Hours Neutral Salt Fog.

Figure 5.7. XPS spectra taken from the top surface of IVD coated steel with and without various plasma coating. 100 
Figure 5.8. High resolution XPS peak analysis of O 1s peaks from the top surface of IVD coated steel with plasma coating. 101

Figure 5.9. High resolution XPS peak analysis of Si 2p peaks from the top surface of IVD coated steel with plasma coating. 102 Figure 5.10. High resolution XPS peak analysis of C 1s peaks from the top surface of IVD coated steel with plasma coating. 102

Figure 6.1. Photograph of a prototyped plasma brush with ceramic nozzle.

Figure 6.2. A depiction of a nozzle insert.

Figure 6.3. Depictions of nozzles created for the plasma brush; (left) a square glass insertable nozzle and (right) circular glass insertable nozzle.

Figure 6.4. Depictions of the prototyped brush shaft (left) and collar nut for holding the nozzle in place (right).

Figure 6.5. Depictions of the plasma brush nozzles used in the sterilization experiments; (left) the straight nozzle and (right) the $45^{\circ}$ nozzle.

Figure 6.6. Depictions of nozzles designed for rat trials.

Figure 6.7. Survivability of L. acidophilus seeded on HA discs with different bacterial seeding concentrations and plasma exposure time.

Figure 6.8. Survivability of $S$. mutans seeded on HA discs with different bacterial seeding concentrations and plasma exposure time.

Figure 6.9. Survivability of L. acidophilus seeded on HA discs with high bacterial seeding concentrations at varying plasma exposure time.

Figure 6.10. SEM images of the high seeding concentration of L. acidophilus cells on HA discs with no treatment (top) and after 10 seconds plasma treatment (bottom). 123 
Figure 6.11. SEM images of the medium seeding concentration of L. acidophilus cells on HA discs without treatment (top-left), after 3 seconds plasma treatment (top-right), after six seconds plasma treatment (bottom-left), and after 10 seconds plasma treatment (bottomright)

Figure 6.12. SEM images of the high seeding concentration of $S$. mutans cells on HA discs without treatment (left) and after 10 seconds plasma treatment (right).....

Figure 6.13. SEM images of the medium seeding concentration of S. mutans cells on HA discs without treatment (left) and after 10 seconds plasma treatment (right).

Figure 6.14. SEM images of the high seeding concentration of L. acidophilus cells (left) and S. mutans (right) on HA discs after 10 seconds plasma treatment.

Figure 6.15. Ozone creation with different amounts of oxygen added near the ground electrode.

Figure 6.16. A depiction of the electrode and gas line set up with respect to the plasma brush nozzle. 128

Figure 6.17. Survivability medium seeding concentration of L. acidophilus seeded on HA discs with different plasma exposure times and oxygen conditions. 128

Figure 6.18. Survivability medium seeding concentration of $S$. mutans seeded on HA discs with different plasma exposure times and oxygen conditions.

Figure 6.19. Optical fiber pointed toward the plasma brush running parallel with the blade.

Figure 6.20. Optical emission spectra of argon plasma. 
Figure 6.21. Light emitted from the plasma at the $777 \mathrm{~nm}$ monatomic oxygen peak with $\mathrm{O}_{2}$ addition near the ground electrode at $0 \mathrm{sccm}$ addition (left), $5 \mathrm{sccm}$ addition (middle), and 50 sccm addtion (right) as observed through a $780 \mathrm{~nm}$ center wavelength bandpass filter. ..... 132 Figure 6.22. Light emitted from the plasma at the $777 \mathrm{~nm}$ monatomic oxygen peak with $\mathrm{O}_{2}$ addition as mixed at $0 \mathrm{sccm}$ addition (left), $5 \mathrm{sccm}$ addition (middle), and $50 \mathrm{sccm}$ addtion (right) as observed through a $780 \mathrm{~nm}$ center wavelength bandpass filter...... 132 Figure 6.23. Light emitted from the plasma at the $777 \mathrm{~nm}$ monatomic oxygen peak with $\mathrm{O}_{2}$ addition as mixed at $0 \mathrm{sccm}$ addition (left), $5 \mathrm{sccm}$ addition (middle), and $50 \mathrm{sccm}$ addtion (right) as observed through a $780 \mathrm{~nm}$ center wavelength bandpass filter. 133

Figure 6.24. Optical emission spectroscopy of plasma during sterilization. 134 Figure 6.25. OES spectra of plasma during sterilization of S. mutans. 134 Figure 6.26. OES spectra of plasma during sterilization of L. acidophilus. 135 Figure 6.27. Volume fraction of argon dispersing through air at atmospheric pressure as simulated by computation fluid dynamics (left); a photo of the plasma brush (right). 136 Figure 6.28. CFD simulation with $\mathrm{O} 2$ injected near the ground electrode; volume fraction of argon (left) and volume fraction $\mathrm{O} 2$ (right). 137

Figure 7.1. A depiction of the microchannel in relation to the plasma plume (not to scale) when treated with electrodes transverse to the microchannel (left) and in-line with the microchannel (right); bacteria represented as spheres. 148

Figure 7.2. Plasma deactivation effect on L.acidophilus seeded on dentin and HA with initial cell densities shown in the legend $(\mathrm{cfu} / \mathrm{mL})$. 149 Figure 7.3. Plasma deactivation effect on S. mutans seeded on dentin and HA with initial cell densities shown in the legend $(\mathrm{cfu} / \mathrm{mL})$. 149 
Figure 7.4. SEM of dentin surfaces seeded with L. acidophilus (left) and S. mutans (right) before plasma treatment.

Figure 7.5. SEM of dentin surfaces seeded with L. acidophilus (left) and S. mutans (right) after 15 seconds plasma treatment.

Figure 7.6. SEM cross-section views of dentin seeded with L. acidophilus (left) and $S$. mutans (right) after 15 seconds plasma treatment.

Figure 7.7. SEM of L. acidophilus seeded on glass cover slips after plasma treatment with electrodes transverse to the microchannel; anode (left) and cathode (right); top of microchannel upper left.

Figure 7.8. SEM of L. acidophilus seeded on glass cover slips after plasma treatment with electrodes in-line with the microchannel; near the surface (left) and $0.064 \mathrm{~mm}$ from the surface (right). 


\section{LIST OF TABLES}

Table 3.1. Hydrogen embrittlement test results for TMS plasma nanocoated, chromate conversion coated, and bare IVD Al coated high-strength steel specimens.

Table 3.2. Calculated total time to failure for hydrogen embrittlement of TMS plasma nanocoated, chromate conversion coated, and bare IVD Al coated high-strength steel specimens.

Table 3.3. Hydrogen re-embrittlement test results for TMS plasma coated IVD Al high-

strength steel specimens in various corrosive solutions.

Table 3.4. Electrochemical measurement results of TMS plasma coated and bare IVD Al coated steel specimens using electrochemical polarization.

Table 3.5. Atomic concentration percentage of top surface of TMS plasma coated and bare IVD Al coated steels. obtained by XPS analysis.

Table 4.1. Results of several IVD trials (Plasma: $30 \mathrm{sccm}$ Ar, $2.54 \mathrm{kV}, 6.5 \mathrm{~mA}$ to $0.05 \mathrm{~mA}$ :

IVD: 550A)

Table 4.2. Results of several IVD trials with cooling cycles between coating cycles (Plasma:

$30 \mathrm{sccm}$ Ar, $2.54 \mathrm{kV}, 6.5 \mathrm{~mA}$ to $0.05 \mathrm{~mA}$ : IVD: $550 \mathrm{~A})$ 70

Table 4.3. Thickness measurements for test panels at locations shown in Figure 4.28........ 75

Table 4.4. Atomic concentration percentage of IVD Al coating surfaces obtained by EDS. 80 Table 5.1. Procedure to coat IVD Al coated steel with TMS plasma coating with and without

oxide layer. 88

Table 5.2. Electrochemical measurements of IVD coated steel with and without various plasma coating based on anodic polarization. 93 
Table 5.3. Atomic concentration percentage of IVD coated steel with and without various plasma coating obtained by XPS analysis. 


\title{
ABSTRACT \\ Investigation of Plasma/Ion Vapor Deposition (IVD) Hybrid Coatings for Corrosion Protection and Plasma Destruction of Oral Bacteria
}

\author{
Adam Blumhagen
}

Dr. Qingsong Yu, Dissertation Advisor

This dissertation reports the research results obtained in investigating low pressure plasma coatings for corrosion protection of aluminum ion vapor deposition (IVD) coated high-strength steels and in studying atmospheric non-thermal plasmas for destruction of oral bacteria.

The objective of investigating plasma coatings for protection of IVD coated highstrength steel is to reduce damage from corrosion and prevent its hydrogen re-embrittlement that often occurs in aircraft. In this study, we completed the design and construction of a hybrid IVD/plasma coater, which has the capability of producing both aluminum IVD coatings and plasma coatings of trimethylsilane (TMS). Both IVD Al coating and TMS plasma coatings were successfully prepared by the hybrid coater. The coating properties of the resulted IVD coating and TMS plasma coating were characterized and evaluated. Corrosion property evaluation using potentiodynamic polarization technique indicated that TMS plasma coated IVD specimens showed a decrease in the magnitude of corrosion potential and a decrease in corrosion current compared to bare IVD, and thus better corrosion resistance. Corrosion test results from $\mathrm{SO}_{2}$ salt spray test showed that a similar amount of corrosion products were found with the plasma coated IVD specimens as their controls, i.e., 
chromate conversion coated IVD samples, and did not form visible amounts of red rust after 504 hours neutral salt fog. Hydrogen re-embrittlement testing demonstrated that TMS plasma coated IVD specimens gave better test results as compared with bare IVD samples. This result is believed to be due to the improved corrosion resistance of the TMS plasma coated IVD specimens and thus less amount of hydrogen formation as a corrosion byproduct. It was further found that IVD Al coating should be exposed to oxygen or air in order to create an oxide layer before organosilane coatings are applied for stronger corrosion resistance. TMS plasma coating did not cause contamination to IVD. Likewise, the application of IVD in the chamber prior to TMS plasma coating does not affect the barrier properties of the TMS plasma coating.

To explore the potential application of non-thermal atmospheric plasmas in the dental field, plasma treatment effects using a plasma brush on disinfection of common oral bacteria were studied in terms of bacterial concentration, supporting media, plasma chemistry, and plasma exposure time. In the first set of oral disinfection experiments, Gram-positive oral bacteria Lactobacillus acidophilus and Streptococcus mutans were seeded on hydroxyapatite disks that were used as a tooth enamel analogue. By altering bacterial seeding density, a physical shadowing effect of bacterial cells was observed. The experimental data indicated that a plasma exposure time of 13 seconds effectively killed all bacteria when concentrations were less than $6.9 \times 10^{6} \mathrm{cfu} / \mathrm{cm}^{2}$ for L. acidophilus and $1.7 \times 10^{7} \mathrm{cfu} / \mathrm{cm}^{2}$ for $S$. mutans. At higher bacterial concentrations of $1.2 \times 10^{8}-2.5 \times 10^{8} \mathrm{cfu} / \mathrm{cm}^{2}$, a $1.5 \log$ reduction in bacterial concentration was observed in the first 7 seconds, but nearly no further reduction in bacterial population was achieved after 20 seconds. Deactivation was determined to be caused by physical bombardment by energetic species. 
The disinfection experiment was repeated on dentin as the supporting media. During the first six seconds, deactivation rates were similar to that as performed on HA. After six seconds, deactivation began to plateau and full disinfection was not achieved. Upon further SEM examination, bacteria were observed within dentin tubules. Plasma treatment was unable to affect these deep lying bacteria. Bacteria were also seeded on glass cover slips held apart to create a microchannel and plasma treatment was performed with electrodes both transverse and in-line to the microchannel. SEM was again performed to examine the location of cell lysis. No disinfection was observed when the plasma treatment was performed with electrodes transverse to the microchannel. Deactivation was observed when plasma treatment was performed in-line with the microchannel. This line-of-sight deactivation result is further evidence that bombarding species were the cause of deactivation. These experiments have shown the effectiveness and geometric dependence of plasma deactivation of oral bacteria. 


\section{Chapter 1 General Introduction}

In the past few decades, plasma engineering has found a niche in the tailoring of surface and interfacial properties of a variety of materials. Although there have been many studies in plasma physics, the complicated nature of plasmas and plasma chemistry leave much knowledge to be gained through experiment.

The objective of this dissertation is to conduct research on low temperature plasma processing in several emerging applications. To achieve this, low pressure direct current (DC) plasma coatings were investigated. Further, a brush-shaped atmospheric plasma brush was constructed and its disinfecting characteristics were examined.

In chapter 2, a brief background on the physics of plasmas, its uses, and research trends in the use of plasmas in industry is discussed.

In chapter 3, a plasma coating is applied on top of ion vapor deposited (IVD) aluminum coated steel substrates for corrosion and hydrogen re-embrittlement resistance. The plasma coating is characterized by X-ray photoelectron spectroscopy and energy dispersive spectroscopy. The plasma coating is comprised of a network of cross-linked $\mathrm{Si}_{\mathrm{x}} \mathrm{C}_{\mathrm{y}} \mathrm{O}_{\mathrm{z}}$. Its performance as a hydrogen re-embrittlement resistive coating is evaluated by tensile testing in various embrittling fluids. The theory is that the film will act as a barrier to diffusing corrosive agents. Less corrosion means the decrease in hydrogen caused by reduction of water and therefore a decrease in hydrogen uptake and resultant hydrogen reembrittlement. Further the plasma coating acts as a barrier to impede diffusion of atomic hydrogen into the IVD Al and underlying steel. 
In chapter 4 , the construction of an IVD reactor chamber is described. The IVD chamber is a hybrid IVD and plasma coating reactor. The IVD coating process lends itself well to the incorporation of plasma coating. Both processes occur under vacuum, therefore few process and equipment changes are necessary for implementation. Plasma parameters are determined for controlled coating thickness. In chapter 5, the coatings produced by the hybrid reactor were evaluated for corrosion performance and coatings were characterized by various spectroscopic methods. By developing a single chamber for IVD and plasma coating, both processes may be performed without breaking vacuum. Because of this, it was possible to apply plasma coatings to the IVD Al with and without an oxide layer. This allowed for the determination of the effect of the oxide layer developed in atmosphere at the plasma coating/IVD coating interface as it pertains to corrosion resistance. Potentiodynamic polarization and 504 hour salt spray tests were used to evaluate the plasma coating's corrosion resistance. The plasma coating is characterized for composition and structure to explain the corrosion results. It is assumed the oxide layer on aluminum is essential to corrosion resistance due to its ability to passivate the underlying aluminum. Also, an oxide layer is necessary for adhesion between the plasma coating and IVD surface. Contamination of the reactor chamber and subsequent IVD coatings is also studied. A small amount of effluent material is produced during plasma coating in the anode dark space region of a parallel plate glow discharge. The low flux of material compared to the high flux of IVD overspray is thought to have minimal effect on IVD coating composition and quality.

Atmospheric plasmas used in the disinfection of oral bacteria for caries prevention and during caries restoration are emerging fields of study. In chapter 6 , an atmospheric pressure plasma brush is used for sterilization of some common oral bacteria. To explore the 
potential application of non-thermal atmospheric plasmas in the dental field, plasma treatment effects using a plasma brush on disinfection of common oral bacteria were studied in terms of bacterial concentration, plasma chemistry, and plasma exposure time. In the first set of oral disinfection experiments, Gram-positive oral bacteria Lactobacillus acidophilus and Streptococcus mutans were seeded on hydroxyapatite disks that were used as a tooth enamel analogue. The experimental data indicated that a plasma exposure time of 13 seconds effectively killed all bacteria when concentrations were less than $6.9 \times 10^{6} \mathrm{cfu} / \mathrm{cm}^{2}$ for L. acidophilus and $1.7 \times 10^{7} \mathrm{cfu} / \mathrm{cm}^{2}$ for $S$. mutans. At higher bacterial concentrations of $1.2 \times 10^{8}-2.5 \times 10^{8} \mathrm{cfu} / \mathrm{cm}^{2}$, a $1.5 \log$ reduction in bacterial concentration was observed in the first 7 seconds, but nearly no further reduction in bacterial population was achieved after 20 seconds. SEM showed plasma deactivation occurred mainly on the top surface of the bacteria supporting medium, while shadowing effects from the resulting bacterial debris reduced plasma deactivation of the underlying bacteria. Optical emission spectroscopy (OES) of the plasma brush did not show many reactive oxygen species. Small amounts of monatomic oxygen were observed. Digital photographs were also taken through a bandpass filter at the monatomic oxygen $777 \mathrm{~nm}$ characteristic peak. Addition of oxygen near the ground electrode did not cause an increase in the observed peak. An ozone detector measured an increase in ozone when oxygen was added near the ground electrode, but no increase in deactivation rate was observed. A computational fluid dynamics simulation was performed to estimate the chemical concentration and distribution within the plasma brush and around the sample surface. A boundary layer was observed near the supporting media surface which indicated little diffusion of oxygen to the surface and therefore little likelihood of deactivation from reactive oxygen species. (Note: fluid dynamics and not plasma dynamics were simulated.) 
No decrease in deactivation rate was observed when performing the plasma treatment in a reduced oxygen environment.

The disinfection experiment was repeated on dentin as the supporting media. During the first six seconds, deactivation rates were similar to that as performed on HA. After six seconds, deactivation began to plateau and full disinfection was not achieved. Upon further SEM examination, bacteria were observed within dentin tubules. Plasma treatment was unable to affect these deep lying bacteria. Bacteria were also seeded on glass cover slips held apart to create a microchannel and plasma treatment was performed with electrodes both transverse and in-line to the microchannel. SEM was again performed to examine the location of cell lysis. No disinfection was observed when the plasma treatment was performed with electrodes transverse to the microchannel. Deactivation was observed when plasma treatment was performed in-line with the microchannel. This line-of-sight deactivation result could be explained by penetration of cells by bombarding charged species, but could also be due to non-penetrating charged species. These experiments have shown the effectiveness and geometric dependence of plasma deactivation of oral bacteria. 


\section{Chapter 2 Literature Review}

\subsection{Introduction}

Plasma occurs naturally on earth as lightning and the aurora borealis, and it is $99 \%$ of visible matter in the universe [1]. Although plasmas were formed in laboratories as far back as the 1700's by G.C. Lichtenberg, until recently, its uses in industry have been limited by the need for reduced pressure for many applications [2]. Man-made plasmas are spread throughout much of the industrialized world most widely seen by the public in the form of fluorescent lights and plasma screen televisions, but they have also found their place in industrial applications. This chapter gives a brief description of plasma and its distinct forms, some of plasma's major uses, and research trends.

\subsection{Background}

A mixture of positively and neutrally charged atoms with negatively charged electrons is termed plasma, the fourth state of matter. The creation of charges may arise from some type of perturbation capable of liberating one of the atom's constituent electrons. This may happen: kinetically, such as the plasma that occurs at the nose cone of a high velocity missile; through excitation by electromagnetic radiation such as by laser or UV light; by inelastic collision of electrons and ions accelerated by electric/magnetic field, etc. The sum of the charges is necessarily neutral to be considered plasma.

\subsection{Local thermal equilibrium plasmas}

Plasmas may be divided into equilibrium thermal plasma and non-equilibrium, low temperature plasmas. In thermal plasmas, temperatures of atoms and electrons achieve a 
local thermal equilibrium (LTE). The inherent high temperature, ranging between 4,000 K to $20,000 \mathrm{~K}$, has been found mostly useful in material destruction such as in arc cutters and toxic chemical destruction [3]. Other applications include thermal plasma spraying particularly high temperature ceramic, production of fine nanoparticle sized powders, and for the excitation of molecules to emit light for optical emission spectroscopy [4,5]. Plasmas used in magnetic confinement fusion reactors will not be discussed here. Plasma spraying is a widely used industrial application of thermal plasma and much of the research in thermal plasmas is in this area. If a material can be melted without degradation then it can be plasma sprayed. This is very helpful for high melting temperature materials such as ceramics. The atomic structure of ceramics enables them to maintain strengths at high temperatures; they are hard and therefore wear resistant, and highly non-reactive. These properties are useful in cutting tools, turbine components, bearings, orthopaedics, etc. A ceramic's superior mechanical strength results in machining being very difficult. Also, ceramics suffer from low shear strengths and brittle failure; therefore it has become a standard practice to apply thin coatings to pre-machined parts. A fine ceramic powder is sprayed at the part while being heated by the plasma to increase interparticle diffusion, i.e. sintering. Plasma can efficiently generate high temperatures in a localized region. This makes for supersaturation of vapor species resulting in high condensation rates. Most research in this area is concentrated on coating quality, deposition of new materials such as superconducting ceramics, and adhesion of coatings [5,6]. Some plasma sprayed coatings of recent interest are in the area of implants for better biocompatibility $[7,8]$. The use of non-LTE plasma is the main focus of this dissertation. No further discussion on thermal plasmas will be presented. 


\subsection{Non-Local Thermal Equilibrium Plasmas}

Thermal plasma spraying research is limited in that it typically deals with the limited number of chemistries that do not degrade at high temperatures. On the other hand, a vast number of chemistries are available for complex reactions with non-LTE plasmas. Unfortunately, the low pressure environment required for low temperature non-LTE plasma hinders both applications for large parts and for high throughput of small parts. Further, the low pressure environment means small concentrations of chemical species and therefore low deposition rates, i.e. on the order of $1-100 \mathrm{~nm} / \mathrm{min}$ for typical non-LTE coatings [9] compared to thermal plasma spraying typically on the order of $10^{8}$ particles/sec at $30 \mu \mathrm{m}$ average particle diameter [10]. In contrast, plasma spray rate is usually only limited by the heat dissipation rate of the substrate and desired coating quality. Some of the most important economic uses of non-LTE plasma are: cleaning of surfaces, liquids, and gases; increasing wear/scratch resistance and corrosion protection (for cutting tools and high performance engines); plasma disinfection; plasma modification of polymers used in textiles (such as for oil and water resistance, adhesion, increasing depth of dyeing, etc.); microstructuring (especially for silicon based microchips); decreasing fluid permeability in the food packaging industry; and production of biocompatible and antifouling coatings for the biomedical, i.e. orthopaedic and endoscopic uses [2,4,11].

Lower temperature non-LTE plasma is an area of great research since a large variety

of chemical species may be employed while maintaining low bulk temperatures. At low pressures, electrons do not efficiently transfer kinetic energy to heavy particles such as atoms, ions, and molecules. Instead, electrons tend to distribute their kinetic energy amongst themselves. When placed in an electric field, the electrons gain more energy and reach 
higher temperatures. In a non-LTE plasma, electrons are accelerated to high temperatures, above $10^{4}-10^{5} \mathrm{~K}$, while atoms may remain near ambient temperature. Ionization may occur from a number of collision processes determined by the pressure of the system, electrode spacing, and frequency of the power supplied. High intensity light may be released during de-excitation of an electron giving the characteristic glow associated with plasmas. Negative ions may also be created due to electron capture. This typically only occurs for very electronegative molecules coming in contact with low temperature electrons. A molecule may store energy through a number of modes: rotational, vibrational bond stretching, translational, and electronic excitation [12]. These energies may be absorbed by a neutral atom also causing ionization to occur. Electron-impact ionization arises from electrons colliding with atoms causing the ejection of an electron. This electron can be accelerated by an electric or magnetic field to cause further ionization events. Typically plasmas are powered by alternating frequencies in the radiofrequency (RF) and microwave frequency range. Oscillation in the direction of the electric field causes an electron to accelerate back and forth. When an electron collides with a neutral atom, the electron may impart some of its energy to the atoms causing the neutral atom to be excited. The electric field may then oscillate to cause the electrons to flow in the opposite direction causing more collisions to occur. In this manner, an electron's energy does not need to be above the ionization energy of the neutral atom in question due to the pre-excitation of the neutral atom from its ground state due to electron collision. Also, neutrally charged species that are now in an excited state may collide with each other causing ionization, known as penning ionization. The net result is the electron temperature in $\mathrm{RF}$ driven atmospheric plasmas tend to be low, i.e. on the order of 1-10 eV [13]. In contrast, in a DC plasma source, electrons do not have translational 
oscillations and therefore electron-neutral collisions do not cause ionization as efficiently. Therefore, electron temperatures of DC plasmas tend to be higher than that of RF driven plasmas [14]. The standard way to create a large low temperature plasma, i.e. on the order of up to cubic meters, is by using a large vacuum chamber. The reduced pressure increases the mean free path compared to the discharge length effectively reducing the probability of momentum transfer from electrons to atoms. Since the relatively heavier atoms maintain a low temperature, the bulk temperature remains low. A low temperature non-equilibrium plasma may be created even at atmospheric pressures, but the discharge length must remain small. The temperature difference between electrons and neutral particles for conventional weakly ionized plasma is proportional to the square of the ratio of the electric field to the pressure [9]. The current density in a plasma scales with the pressure, therefore higher pressures result in smaller cross sectional areas of plasma. A limit occurs where the discharge changes from a non-LTE to LTE [14]. This is called a glow-to-arc transition, where ionization from atom-atom collisions begins to occur at a much higher rate.

The electron and ion density, temperature, and distribution within a plasma will depend on the electrode geometry, gas flow dynamics and chemistry, frequency of the electric and magnetic fields, and pressure of the system. Ions accelerated to sufficient temperatures will impact the cathode creating secondary electrons from the cathode to be released into the plasma. Electrons are accelerated continuously in the electric field away from the cathode until it obtains sufficient energy to cause electron-impact ionization releasing more electrons and reducing the average electron temperature. The density of electrons in this region becomes relatively dense. The characteristic glow occurs near this region due to the high temperature of electrons. In a DC discharge, this develops columns of 
illumination. A sheath, or boundary layer between plasma and the cathode, will develop. The sheath will govern the plasma's interaction with the substrate. The plasma sheath tends to grow with increasing power density and decrease with increasing pressure $[16,17]$. In the cathode region, the electric field strength is large and therefore the electron and ion densities are small. With alternating frequencies, layers of positive and negative charge density appear called double layers. These grow, go extinct, and move depending on the frequency [17].

\subsubsection{Non-LTE plasma coating}

Non-LTE plasmas are sometimes called glow discharges instead of plasma, but many use the terms synonymously. Non-thermal glow discharges are of particular interest for superficial modification of solid surfaces since their low average temperatures result in less bulk heating of substrate materials compared to chemical vapor deposition (CVD). Research in non-LTE plasmas centers around chemical processes where wet and thermally activated chemical methods are unsatisfactory. The inherent advantages plasma processing has over typical wet/thermal chemical methods are: the ability to direct chemical species; the ability to bombard and even penetrate into substrates while maintaining low substrate temperatures; the ability to create high temperature gradients for localized heating; little effluent material and therefore less waste; wet chemical processes produce porous materials and often require curing for increased densification while plasmas are able to create more highly cross-linked and highly adherent coatings [16]; new chemistries not standard to wet chemicals may be used, for instance plasma is capable to polymerize inert gases such as methane and benzene; the ability to incorporate new functional groups into inert materials with minimum bulk degradation [1,16]. The structure, composition, mechanical and chemical properties of a plasma derived coating is dependent on a number of factors: 
1) the monomer or feed gas and its flow rate;

2) geometry of the reactor, electrodes, and substrate;

3) frequency of the discharge;

4) working pressure; and

5) substrate temperature.

Three temperatures: electron temperature, ion temperature, and excited neutral temperature are all necessary to characterize a plasma's temperature. The chemistry of a coating may depend on the energy distribution of these species and the collision cross-sections of the particles present. Modifications may be: removal of surface atoms by plasma etching or ablation; deposition of thin films; and finally chemical and/or physical modification without removal or deposition of material. Further, the determining factors of these modifications rely on the mode of consumption of gases comprised by the plasma [16]. Gases may be chemically nonreactive, chemically reactive but not polymer forming, and chemically reactive and polymer forming. Chemically nonreactive gases include noble gases that may ionize but cannot chemically bond. Chemically reactive but not polymer forming gases consist of gases that cannot form deposits by themselves alone, such as $\mathrm{O}_{2}, \mathrm{~N}_{2}, \mathrm{CO}, \mathrm{NH}_{3}$, $\mathrm{CF}_{4}$, etc., but may incorporate themselves into a coating of other materials. Finally, polymer forming are gases capable of forming solid deposits without the need for other materials. Plasma coating is a competitive process between ablation and deposition. A substrate material may be sputtered by a plasma and re-deposited onto the substrate. The characteristics of the plasma coating system needs to be finely tailored for the desired result. 
For instance, low molecular weight films and small granules may deposit in a low adhering oily coating, typically unwanted in most industrial applications.

Dissociation is the breaking apart of molecules in a plasma due to collision. Dissociation tends to be more probable than ionization since interatomic bond strength tends to be low in many organic molecules compared to the energy required to remove an electron [18]. Homolytic bond dissociation tends to be favored due to the low dissociation energy, i.e. 3-5 eV, compared to heterolytic dissociation with bond energies in the range $10-13 \mathrm{eV}$ [12]. Electron ejection occurs at a $\sigma$-bond, $\pi$-bond, or lone electron pair. Typical ionization energies are in the range of 7-15 eV. Electrons from $\sigma$-bonds being the least likely and lone electrons being most likely to be ejected. Ionization energy of molecules tends to decrease with increasing size and number of $\pi$-bonds due to the relatively low bond strength of $\pi$ bonds to $\sigma$-bonds and the ease of charge distribution and stabilization with large molecules [12]. Ionization of the molecule without dissociation may occur. In general, this causes stretching of other bonds and therefore less energy for these bonds to break during other excitation events effectively dissociating the molecule.

Low temperature plasmas created by an electric field often do not truly have a net neutral charge typically due to secondary electron emission from bombarded surfaces, especially at the cathode in DC glow discharges [19]. These electrons are accelerated in the electric field toward the anode. Various gaseous compounds may be pumped into the discharge. The compound may be dissociated by the bombarding atoms and electrons or due to scission from high energy light, e.g. statistically weaker bonds broken first. The result is a compound with an unpaired electron that reacts in a way similar to free radical addition 
polymerization initiators. Free radicals may react with each other, the original unfragmented compound, and substrates. In most cases, the objective is to form a dense thin film that adheres well with the substrate and has specific functional groups available for further reactions. Also, a large number of dangling bonds may remain on the coating surface which may bond with subsequent coatings forming a strong adherence between coatings. It should be noted, there is various nomenclature for the creation of plasma coatings. Some in the industry use the term plasma polymerization (PP). By this, it is meant any process by which the molecular weight of a high molecular weight structure is significantly increased. As these coatings do not have a back-bone typically found in conventional polymerization methods, they are not truly polymers. From this point forward, this paper simply describes the resultant coating as a plasma coating instead of PP. Some of the ions may penetrate the substrate and become part of the substrate, i.e. ion implantation. The result can be a gradient between substrate and coating. This may cause better adhesion and less interfacial stress due to the difference in thermal expansion coefficients. An important example of a non-LTE plasma is for corrosion resistant thin films. In recent research, direct current glow discharges have shown their superior adhesion and barrier properties in protecting steel and aluminum alloys from various corroding liquids [Yasuda, Iriyama]. Plasma will fragment most any organic compound, and therefore coatings may be created from a wide variety of gas phase organic monomers. Some typical monomers are styrene, ethylene, naphthalene, methane, and various fluorocarbons and organosilanes [18,21-23]. Plasmas are able to create high concentrations of chemically reactive species, therefore dense, highly crosslinked films may be created [9]. Coatings are composed of strong covalent bonds and can be made to be insoluble to water and organic solvents and remain stable up to $800 \mathrm{~K}$ in air $[18,9]$. The 
wettability of plasma coatings can be tailored by the plasma chemistry. Hydrocarbons tend to have high wettability while fluorocarbons and organosilicates tend to have low wettability. In general, a coating can be made more wettable by incorporating oxygen into the coating.

\subsubsection{Plasma etching}

One of the most abundant uses of plasmas is for microstructuring such as that used in the semiconductor industry. Etching may be produced by two mechanisms: physical sputtering by ion bombardment and chemical etching. $\mathrm{O}_{2}$ plasmas used in plasma ashing have an advantage to wet chemical methods for removing silicon during photolithography of microchips [24]. In reactive ion etching, the controlled directionality of bombarding ions and reactive neutrals allows better accuracy due to less undercutting of lower layers. Also, controlled directionality allows high aspect ratio trenches to be etched between interconnects. PECVD has been used to deposit low-k dielectric materials as interlayer insulators which results in less cross-talk between neighboring circuits and less resistance-capacitance delay. Another use of plasma greatly used in the microelectronics industry is plasma sputtering. Plasma sputtering may be used to apply the thin metal coatings that make up the circuits as well as a method to remove the photoresist. In plasma sputtering, a surface is held at a high negative potential in an inert gas. Ions are formed and attracted to the surface causing ejection of surface atoms. These atoms may then deposit on other substrates. Reactive gases may be inserted into the plasma, i.e. reactive plasma sputtering. The resulting coatings can be a variety of chemistries including various nitrides, oxides, carbides, etc [25-27]. Research in the microelectronic field is mostly concerned with: increasing aspect ratios of etched trenches, reducing damage to low-k materials during etching, and gas chemistry selection for biased etching of various materials, and the effect of dusty plasmas [24]. 


\subsubsection{Plasmas in the medical field}

An emerging use for plasmas is in the medical field. Prestigious journals have dedicated full sections on plasma medicine and new journals Plasma Medicine and Clinical Plasma Medicine have recently appeared [28]. Both hot LTE and cold non-LTE plasmas have found utility in the medical field. Thermal equilibrium plasmas are characterized by very high temperatures, so their uses are mainly in the areas of surgical cauterization, ablation, and cutting of tissues. It was observed that atmospheric plasmas were capable of producing reactive oxygen species which were already widely used for disinfection by wet chemical methods. The ability of plasmas to create ROS made them an interesting area for plasma disinfection. Indeed, plasma disinfection is one of the most investigated biological applications of cold plasmas [9]. Gases without germicidal properties may be made biocidal during plasma discharge, with few short lived effluent biocidal residual particles. Plasma has been used to sterilize medical devices rapidly and at low temperatures [29]. Most conventional sterilization methods require dry heat around $170{ }^{\circ} \mathrm{C}$ or moist heat around 121 ${ }^{\circ} \mathrm{C}$ such as by autoclave. In recent years, many new methods of creating non-LTE plasmas at atmospheric pressures have been developed. These include microdischarges, dielectric barrier discharges, plasma jets, plasma needles, and the one atmosphere uniform glow discharge. These are created with discharge power sources of direct, pulsed, and alternating currents in the kilo-, radio-, and mega- hertz range frequencies [30-34]. These plasma sources tend to create plumes or jets of plasma inside a tube which is pushed out of the tube by flowing gas. Plasma plumes tend to be of a needle type discharge, i.e. a pointed cathode electrode with gas surrounding, or jet type discharge, i.e. two parallel electrodes with gas flowing between. If the cathode is made sharp, an increase in electric field strength near the 
tip will occur which allows discharge at low operating power and therefore low bulk temperatures. The creation of these low temperature discharges allows for the treatment within the body without damaging sensitive soft tissues [28]. This has become of particular interest recently for use in the field of oral disinfection. Many researchers have investigated non-LTEs for the deactivation of microorganisms. Plasma treatments have been shown to be effective at rapidly deactivating bacteria, spores, and prions presumably by the creation of reactive oxygen species, charged particle bombardment, electroporation, and UV radicals [9,34-37]. For bacteria, deactivation tends to occur from damage to the cell wall or to DNA. The specific mechanisms of deactivation remain in debate making deactivation of oral bacteria an intriguing area for further research. Also, there exists no wet chemical method that guarantees protection against oral caries. Hydrogen peroxide, alcohol, and antibiotics have all been used in conventional mouthwashes and oral rinses, but their use alone is not sufficient in stopping tooth decay. This leaves an opportunity for other methods such as plasma to aid in the protection against oral caries.

Another major use of plasmas, not covered in this dissertation, is for the creation of coatings for orthopedic devices. Implants have historically suffered from a wide array of problems: eliciting inflammation, malignant neoplasm, hypersensitivity, systemic effects over time, etc. [11,38,39]. Research is ongoing to increase biocompatibility. One major research topic is the use of PECVD of diamond like carbon (DLC). DLC has been found to be non-cytotoxic, non-inflammatory, non-thrombotic, wear resistant, corrosion resistant, etc. [40]. Plasma coatings, including DLC, can be made to reduce the release of toxic chemicals such as from corrosion of implants [41]. There is increasingly more work on tailoring the cell growth at the implant surface either by modifying the implant's 
hydrophobicity/hydrophilicity or by the selective attachment of various proteins $[11,39,42]$.

The use of bioresorbable polymers has been of increasing interest in the last decade, but few polymer options exist. Modifying existing polymers with plasma coatings to be more biocompatible may be a better alternative for some applications [42].

\subsubsection{Plasma modification of surfaces}

Lastly, non-LTE plasma can be used to modify surfaces without deposition or removal of material. Bombarding ions may cause surface roughening which may be advantageous in adhesion and wetting of the substrates such as polymers. Also, bombarding ions may increase substrate surface energy. This can change grain structures, porosity, and adhesion in metal coating processes such as ion vapor deposition [43]. For instance, metal may be applied onto substrates in thin coatings by evaporation in a vacuum coating process known as physical vapor deposition. The process typically suffers from an open columnar structure due to a physical shadowing mechanism and crystal formation due to epitaxy and a process known as Oswald ripening. If the substrate is pretreated with a plasma, it may form a more dense amorphous structure while remaining chemically unchanged [44].

\subsection{Plasma simulation}

Plasma processes have largely been developed through costly and time consuming trial and error. This is due to the close relationship between plasma characteristics with specific materials processes coupled with reactor design. Also, use of direct probes is often insufficient due to minute local variations in the plasma that can vary over short time scales. There may also be a great number of chemical reactions within the plasma and between the plasma and substrate which may occur in many complex steps [45]. Biological materials have more complex chemical structures typical inorganic materials making simulation of the 
interaction with plasma very difficult [46]. Numerical simulation for most any plasma processes has yet to be performed. The standard method for modeling the numerous chemical reactions is by particle simulation, i.e. particle in cell (PIC). The motion of each particle is described by Newton's laws under influence of electric and magnetic fields and

charges. Obviously, the number of particles at atmospheric pressure in macroscale plasmas is too large for today's computers. A compromise between accuracy and calculation time must be made. In the future, this will be a great area of research enabling less time designing experiments.

\subsection{Plasma cost benefits}

Plasma processing often offers advantages to wet chemical methods, namely the nonpolluting or low-polluting nature of the plasma process, and the reduction in materials and effluent material treatment costs. For instance, hazardous chemicals such as chromate conversion coatings may be replaced with environmentally benign plasma coating processing without loss of corrosion protection. Further, corrosion resistant plasma coatings may save money compared to electroplated coatings due to the high cost of water treatment and processing time. The upfront costs to incorporate plasma coating into industrial processes may be high compared to electroplating, but these costs are small in comparison to the reoccurring costs for sustained usage of electroplating.

\subsection{Corrosion and Hydrogen Re-embrittlement}

\subsubsection{Corrosion}

In this dissertation, plasma processing is applied as a method to alleviate the problems of corrosion and hydrogen re-embrittlement. Corrosion is an electrochemical process that 
causes a reduction reaction to occur in metals. Reduction of the metal causes the metal to deteriorate effectively weakening the structure to mechanical loads. A common form of corrosion is the red rust formed on steel. Metal coming into contact with water and oxygen creates anodic and cathodic areas. In anodic areas, oxidation of the metal takes place forming $\mathrm{Fe}^{2+}$ ions. Electrons run through the anodic to the cathodic region producing an electrochemical cell. In cathodic areas, oxygen $\left(\mathrm{O}_{2}\right)$ in the water solution moves to the cathode to form hydroxyl ions $\left(\mathrm{OH}^{-}\right)$. This reduction half-cell reaction is:

$$
\mathrm{O}_{2}+2 \mathrm{H}_{2} \mathrm{O}+4 \mathrm{e}^{-} \rightarrow 4 \mathrm{OH}^{-}(1)
$$

The reduction potential being $+0.40 \mathrm{~V}$. Many common metals will form a spontaneous couple with this half-cell reaction. For iron, the corresponding half-cell reaction is:

$$
\mathrm{Fe} \rightarrow \mathrm{Fe}^{2+}+2 \mathrm{e}^{-}(2)
$$

The reduction potential being $-0.44 \mathrm{~V}$. The higher reduction potential for reaction 1 causes the reduction of oxygen to be favored over iron. The combined reaction is:

$$
2 \mathrm{Fe}+\mathrm{O}_{2}+2 \mathrm{H}_{2} \mathrm{O} \rightarrow 2 \mathrm{Fe}(\mathrm{OH})_{2}(3)
$$

In a solution with higher $\mathrm{pH}$, the anodic reaction creates ferric oxide and hydrogen according to:

$$
2 \mathrm{Fe}+3 \mathrm{H}_{2} \mathrm{O} \rightarrow \mathrm{Fe}_{2} \mathrm{O}_{3}+6 \mathrm{H}^{+}+6 \mathrm{e}^{-}(4)
$$

Aluminum is a more reactive metal than iron, having the anode half-cell reaction:

$$
\mathrm{Al} \rightarrow \mathrm{A} 13++3 \mathrm{e}^{-}(5)
$$

The reduction potential being $-1.66 \mathrm{~V}$. Aluminum oxidizes rapidly, but it forms the strong and stable aluminum oxide $\left(\mathrm{Al}_{2} \mathrm{O}_{3}\right)$, which protects the underlying metal from further oxidation. The rate of corrosion is dependent upon the electromotive potential of the 
electrochemical cell as well as the conductivity of the aqueous solution closing the circuit. The conductivity is increased with the presence of dissolved salts, therefore metals in contact with salt water becomes problematic [47]. Coatings such as paint or polymer act as a barrier to electrolytes. These barriers suffer from high porosity, poor contact with the substrate, and little bonding to the substrate resulting in delamination of the coating. There are many forms of corrosion in painted metals but this paper has categorized the corrosion types of interest as damaged and undamaged barrier corrosion (pitting corrosion) [48]. In damaged barrier corrosion, a large amount of electrolyte contacts the metal. In undamaged barrier corrosion, water travels through pores and diffuses through the barrier causing microdelamination. Water must come in large enough quantities to form the electrochemical cell before corrosion begins. In both corrosion types, delamination of the barrier occurs. To stop corrosion, prevention of reactant water, reactant oxygen, electrons, and ions from reaching the reaction site is necessary. An optimum coating would have minimal permeability, i.e. pore size, a minimum electrical conductivity at the metal/coating interface, a poor catalyst to the oxygen reduction reaction, and high adhesion without sensitivity to water [48]. A coating with corrosion inhibitors reduces damaged surface corrosion but suffers from increased pitting corrosion and leaching of the inhibitors out of the coating.

\subsection{Hydrogen Re-embritllement}

A reduction of load strength in a metal can occur without the visible damage associated with corrosion due to the absorption of hydrogen. A propensity of failure at slow strain rates and at elevated temperatures suggests a lattice diffusion mechanism. Hydrogen may form bubbles during solidification of the melt, it may form brittle hydrides at crack tips, and it may cause crack propagation during sustained loads. There is no consensus on the 
mechanisms causing hydrogen embrittlement, but most theories suggest either an internal pressure mechanism or physical adsorption at some form of microflaw [49]. Physical adsorption theory suggests hydrogen will adsorb at crack tips reducing the surface energy thereby reducing the strength of nearby metallic bonds. This theory does not fully account for the preference of metals such as iron to adsorb oxygen instead of hydrogen thereby protecting the metal from hydrogen attack. The internal pressure theory suggests hydrogen adsorbed on metal surfaces will combine to form molecular hydrogen that collects at voids and causes an internal pressure. The internal pressure can cause crack propagation and premature failure. All mechanistic theories agree that absorption of hydrogen causes the premature brittle failure observed. Common causes of hydrogen formation are during electroplating and in-service due to corrosion as described by Equation 4 . 


\subsection{References}

1. Freidrich, J., Plasma Chemistry of Polymer Surfaces: Advanced Techniques for Surface Design (2nd Edition), John Wiley \& Sons, pages 1-49.

2. D’Agostino, R., Favia, P., Oehr, C., Wertheimer, M.R., 2005, "Low-Temperature Plasma Processing of Materials: Past, Present, and Future," Plasma Processes and Polymers, 5, 7-15.

3. Bogaerts, A., Neyts, E., Renaat, G., Van der Mullen, J., 2002, "Gas discharge plasmas and their applications," Spectrochimica Acta Part B, 57, 609-658.

4. Bonnizzoni, G., Vassallo, E., 2002, "Plasma physics and technology; industrial applications," Vacuum, 64, 327-336.

5. Pfender, E., 1999, "Thermal Plasma Technology: Where Do We Stand and Where Are We Going," Plasma Chemistry and Plasma Processing, 19, 1-31.

6. Davis, J., 2004, Handbook of Thermal Spray Technology, Novelty, OH.

7. Liang, H., Shi, B., Fairchild, A., Cale, T., 2004, "Applications of plasma coatings in artificial joints: an overview," Vacuum, 73, 317-326.

8. Sun, L., Berndt, C., Gross, K., Kucuk, A., 2001, "Material Fundamentals and Clinical Performance of Plasma-Sprayed Hydroxyapatite Coatings: A Review," Journal of Biomedical Materials Research, 58, 570-592.

9. Fridman, A., and Friedman, G., 2013, Plasma Medicine ( $2^{\text {nd }}$ Edition), Wiley, Hoboken, NJ.

10. Fauchais, P., Montavon, G., Vardelle, M., Cedelle, J., 2006, "Developments in direct current plasma spraying," Surface \& Coatings Technology, 201, 1908-1921.

11. Chu, P., Chen, J., Wang, L., Huang, N., 2002, "Plasma-surface modification of biomaterials," Materials Science and Engineering R, 36, 143-206.

12. Gross, J., 2011, Mass Spectrometry, Springer-Verlag, Berlin, Germany, chap. 2.

13. Schutze, A., Jeong, J., Babayan, S., Park, J., Selwyn, G., Hicks, R., 1998, "The atmospheric-pressure plasma jet: a review and comparison to other plasma sources," IEEE Transaction on Plasma Science, 26, 1685-1694.

14. Choi, J., Iza, F., Lee, J., Ryu, C., 2007, "Electron and ion kinetics in a DC microplasma at atmospheric pressure," IEEE Transactions on Plasma Science, 35, 1274-1278.

15. Kunhardt, E., 2000, "Generation of Large-Volume, Atmospheric-Pressure Nonequilibrium Plasmas, ” IEEE Transactions on Plasma Science, 28, 189-200. 
16. Yasuda, H., 2005, Luminous Chemical Vapor Deposition and Interface Engineering, Marcel Dekker, New York.

17. Gottscho, R., 1987, "Glow-discharge sheath electric fields: negative-ion, power, and frequency effects," Physical Review A, 36, 2233-2242.

18. Yasuda, H., 2005, "Some important aspects of plasma polymerization," Plasma Processes and Polymers, 2, 293-304.

19. Campanell, M., Khrabrov, A., Kaganovich, I., 2012, "Instability, collapse, and oscillation of sheaths caused by secondary electron emission," Physics of Plasmas, 19, 123513-1-123513-11.

20. Iriyama, Y., Ihara, T., Kiboku, M., 1996, "Plasma polymerization of tetraethoxysilane on aluminum granule for corrosion protection," Thin Solid Films, 287, 169-173.

21. Yasuda, H., Yu, Q., 2004, "Creation of polymerizable species in plasma polymerization," Plasma Chemistry and Plasma Processing, 24, 325-351.

22. Yamaguchi, M., Tanaka, A., Suzuki, T., "A support film of plasma-polymerized naphthalene for electron microscopy: method of preparation and application," Journal of Electron Microscopy, 41, 7-13.

23. Niinomi, M., Kobayashi, A., Bell, Shen, M., 1973, "Morphology of plasmapolymerized ethylene," Journal of Applied Physics, 44, 4317-4321.

24. Abe, H., Yoneda, M., Fujiwara, N., 2008, "Developments of Plasma Etching Technology for Fabricating Semiconductor Devices," Japanese Journal of Applied Physics, 47, 1435-1455.

25. Tessier, P.Y., Kre N'guessan, R., Angleraud, B., Fernandez, V., Mubumbila, N., Turban, G., 2000, "Carbon nitride thin films deposited by reactive plasma beam sputtering," Surface and Coatings Technology, 125, 295-300.

26. Sung, Y.M., Kim, H.J., 2007, "Sputter deposition and surface treatment of TiO2 films for dye-sensitized solar cells using reactive RF plasma," Thin Solid Films, 515, 49964999.

27. Kusano, E., Satoh, A., Kitagawa, M., Nanto, H., Kinbara, A., 1999, “Titanium carbide film deposition by DC magnetron reactive sputtering using a solid carbon source," Thin Solid Films, 343-344, 254-256.

28. Kolb, J., Mohamed, A., Price, R., Swanson, R., Bowman, A., Chiavarini, R., Stacey, M., Schoenbach, 2008, "Cold atmospheric pressure air plasma jet for medical applications," Applied Physics Letters, 92, 1-3. 
29. Halfmann, H., Bibinov, N., Wunderlich, J., Awakowicz, P., 2007, "A double inductively coupled plasma for sterilization of medical devices," Journal of Physics D: Applied Physics, 40, 4145-4154.

30. Stark R., Schoenbach K. Direct current high-pressure glow discharges. Journal of Applied Physics 1999; 85: 2075-2080.

31. Dobrynin D., Fridman G., Friedman G., Fridman A. Physical and biological mechanisms of direct plasma interaction with living tissue. New Journal of Physics 2009; 11: 11-22.

32. Goree J., Liu B., Drake D., Stoffels E. Killing of S. mutans Bacteria Using a Plasma Needle at Atmoshperic Pressure. IEEE Transaction on Plasma Science 2006; 34: 13171324.

33. Jiang C., Schaudinn C., Jaramillo D., Webster P., Costerton J.W. In Vitro Antimicrobial Effect of a Cold Plasma Jet against Enterococcus faecalis Biofilms. ISRN Dentistry 2012; 2012: 1-6.

34. Laroussi M., Lu, X. Room-temperature atmospheric pressure plasma plume for biomedical applications. Applied Physics Letters 2005; 87: 113902.

35. Boudam M.K., Moisan M., Saoudi B., Popovici C., Gherardi N., Massines F. Bacterial spore inactivation by atmospheric-pressure plasma in the presence or absence of UV photons as obtained with the same gas mixture. Journal of Physics D: Applied Physics 2006; 39: 3494-3507.

36. Kong, M., Kroesen, Morfill, G., Nosenko, T., Shimizu, T., Van Dijk, J., Zimmermann, J., 2009, "Plasma medicine: an introductory review," New Journal of Physics, 11, 1-35.

37. Yu Q.S., Huang C. Deactivation effects of atmospheric cold plasma brush. Applied Physics Letters 2006; 88: 013903.

38. Oehr, C., 2003, "Plasma surface modification of polymers for biomedical use," Nuclear Instruments and Methods in Physics Research B, 208, 40-47.

39. McLaughlin, J., Maguire, P., 2008, "Advances on the use of carbon based materials at the biological and surface interface for applications in medical implants," Diamond and Related Materials, 17, 873-877.

40. Grill, A., 2003, "Diamond-like carbon coatings as biocompatible materials-an overview," Diamond and Related Materials, 12, 166-170.

41. Liu, X., Chu, P., Ding, C., 2004, "Surface modification of titanium, titanium alloys, and related materials for biomedical applications," Materials Science and Engineering R, 47, $49-121$. 
42. Desmet, T., Morent, R., Geyter, N., Leys, C., Schacht, E., Dubruel, P., 2009, "Nonthermal Plasma Technology as a Versatile Strategy for Polymeric Biomaterials Surface Modification: A Review," Biomacromolecules, 10, 2351-2378.

43. Mattox, D.M., 1973, "Fundamentals of Ion Plating," J. Vac. Sci. Technol., 10, 47-52.

44. Schiller, S., Heisig, U., Goedicke, K., 1975, "Alternating ion plating- A method of highrate deposition," J. Vac. Sci. Technol., 12, 858-864.

45. Sakiyama, Y., Graves, D., Stoffels, E., 2008, "Influence of electrical properties of treated surface on RF-excited plasma needle at atmospheric pressure," Journal of Physics D: Applied Physics, 41, 1-9.

46. Lee H., Park G., Seo Y., Im Y., Shim S., Lee H. Modeling of atmospheric plasmas for biomedical applications. Journal of Physics D: Applied Physics 2011; 44: 1-27.

47. Roberge, P.R., Corrosion inspection and monitoring, Wiley, Hoboken, NJ, chap. 1.

48. Leidheiser, H., 1982, “Corrosion of painted metals-a review," Corrosion, 38, 374-383.

49. Chalaftris, G., 2003, Evaluation of aluminium-based coatings for cadmium replacement, $\mathrm{PhD}$ thesis, School of Industrial and Manufacturing Science, Cranfield University. 


\title{
Chapter 3 Hydrogen Re-Embrittlement Protection of IVD Coated Steel by Plasma Coating
}

\begin{abstract}
This study investigated trimethylsilane (TMS) plasma nanocoatings for protection of ion vapor deposited (IVD) Al coated high-strength steel substrates against hydrogen re-embrittlement. A direct current cathodic plasma deposition process, which is an environmentally friendly coating process, was used to produce TMS plasma nanocoatings on IVD Al coated steel substrates. Based on the testing results using ASTM Method for Mechanical Hydrogen Embrittlement Testing of Plating and Aircraft Chemicals (F519), plasma nanocoatings were not found to cause hydrogen embrittlement. Levels of loading at failure were similar to both IVD Al coated and Iridite conversion coated IVD steel samples. It was also found that TMS plasma nanocoated samples resisted hydrogen re-embrittlement in various embrittling solutions. TMS plasma nanocoated samples passed the 150 hours at 45 percent notch fracture strength, the industry standard for re-embrittlement study. Based on electrochemical measurements using electrochemical polarization, TMS plasma nanocoated samples showed higher corrosion resistance, thereby reduced hydrogen formation through hydrolysis of water, and consequently resulted in less hydrogen re-embrittlement. X-ray photoelectron spectroscopy (XPS) characterization showed that the TMS plasma nanocoating is mainly composed of $\mathrm{Si}, \mathrm{C}$, and $\mathrm{O}$ elements. Corrosion protection achieved by TMS plasma nanocoating could be attributed to the tight three-dimensional network structure of plasma coatings that act as a barrier against diffusing corrosive agents.
\end{abstract}




\subsection{Introduction}

Hydrogen embrittlement and hydrogen re-embrittlement has been an historical concern for high strength steels used in aircraft [1]. The distinction between hydrogen embrittlement and hydrogen re-embrittlement is the time of hydrogen absorption, i.e. during coating or inservice through contact with hydrogen, respectively. There is no general consensus on the mechanisms of hydrogen damage, but it is agreed that these embrittling mechanisms will not occur if hydrogen is not allowed to absorb into the metal bulk [2]. If hydrogen is allowed into the metal, the result is diminished yield strength and localized plasticity [3]. Hydrogen solubility of a metal increases with tensile stress by increasing atomic spacing. Since tensile stress is intensified at crack tips, hydrogen solubility is greatly enhanced causing an increased propensity for crack propagation and premature failure. In-service conditions for load bearing metals, therefore tends to cause a higher propensity for hydrogen re-embrittlement.

Hydrogen may be formed as a byproduct of corrosion. To protect against corrosion, high-strength steel aircraft parts are often coated with a sacrificial metal coating such as aluminum. A thin layer of aluminum may be applied by evaporation of aluminum in a vacuum. For a denser microstructure, during deposition of aluminum, the substrate may be held at a high voltage in noble gas plasmas. This process is known as ion vapor deposition (IVD). The resultant coating remains porous and may suffer an increase in corrosion rate due to galvanic corrosion. To further protect and seal the aluminum coating, a chromate conversion coating is often applied. Chromate conversion coatings are extensively used in the aerospace industry for its good corrosion protection and paint adhesion properties, but chromate coatings, and electroplated metals in general, suffer from hydrogen embrittlement. During pickling of steel parts, to remove contaminants attached to the steel surface, as well 
as during electroplating, hydrogen is absorbed by the steels. Therefore, chromate coatings require heating after application to remove absorbed hydrogen. Sacrificial coatings save the underlying steel from corroding, but corrosion remains in the sacrificial coating and hydrogen formed as a byproduct may still cause hydrogen re-embrittlement $[4,2]$.

Recently, chromate was banned by the EPA because it was found to be carcinogenic, therefore a non-toxic method to make an IVD Al coating resistant to re-embrittlement is very desirable [5]. The aircraft may also be exposed to hydrogen re-embrittling materials inservice such as runway deicer and aircraft cleaners. Embrittlement of IVD Al is highly problematic; IVD Al coated steels have been found to be highly susceptible to hydrogen reembrittlement. Pollock et al. found high-strength steels created 100 times more hydrogen with IVD Al coating than with electroplated cadmium coating. Further, high-strength steel was found to be severely embrittled with hydrogen contents less than a few parts per million [6].

In this paper, TMS plasma nanocoatings, an environmentally friendly alternative coating to chromate conversion coating, were studied for protection of IVD Al coated high-strength steels from hydrogen re-embrittlement. We hypothesize the plasma nanocoating will protect the steel substrate from corrosion, thereby reducing the amount of hydrogen formation and subsequently lessening hydrogen re-embrittlement. Organosilane plasma nanocoatings are becoming a popular method to enhance corrosion protection of aluminum and steel substrates [7-11]. The organosilane used in this research is trimethylsilane (TMS: $\left.\left[\left(\mathrm{CH}_{3}\right)_{3}-\mathrm{SiH}\right]\right)$ has hydrogen in its molecular structure, therefore the coating must be evaluated to ensure no increase in hydrogen embrittlement occurs during deposition process of the plasma nanocoatings. To examine the efficacy of plasma nanocoating for reducing hydrogen re- 
embrittlement, plasma nanocoated and uncoated notched steel specimens were tensile tested in various corroding and embrittling solutions.

\subsection{Experimental}

\subsubsection{Materials}

4340 steel ASTM Type 1a notched tension bars coated with 1100 class 1 IVD Al coating, i.e., 0.01 inch coating thickness, were supplied by The Boeing Company. TMS was procured from Gelest Inc. with $97 \%$ minimum purity. Oxygen $\left(\mathrm{O}_{2}, 99.9 \%\right)$ was procured from Airgas. Argon (Ar, 99.9\%) was procured from Praxair. Gases were used as received without further purification.

\subsubsection{Plasma Reactor}

A bell jar type reactor was used in this study and an MDX-1K magnetron drive (Advanced Energy Industries) direct current (DC) power supply was used as a plasma power source. The system was pumped down to a base vacuum of 1 mTorr using an Edwards EH series vacuum pump consisting of a rotary pump (Model E2M 80) and a booster pump (Model EH500A). Argon and TMS were flowed into the reactor at $1 \mathrm{sccm}$ each and the working pressure was preset at 50 mTorr. A MKS mass flow meter (Model $247 \mathrm{C}$ ) was used for monitoring the gas flow rate and a MKS pressure controller (Model 252) was used to control the gas pressure in the reactor chamber. After the system pressure stabilized to a preset value, the plasma was ignited using the DC power supply operated in voltage control mode. 


\subsubsection{Sample Preparation}

IVD coated steel panels in a dimension of $1 " \times 6 " \times 0.04 "$ and ASTM F $5191 \mathrm{a} .1$ load bar specimens were cleaned by The Boeing Company with an alkaline cleaner and pickling solution, and grit blasted. Specimens were suspended from electrodes in the vacuum chamber and plasma coated. Plasma coating parameters were determined with silicon wafers attached to the panels. The coating thickness was measured using a null-seeking type Ellipsometer (Rudolph Research AutoEL-II).

\subsubsection{Hydrogen Embrittlement}

Twelve ASTM F 519 1a.1 load bar specimens were IVD Al coated in Boeing production facilities and six bars were plasma coated at the University of Missouri. Six bars were retained at Boeing-St. Louis; three received no further treatment, and the remaining three load bars were conversion coated with MacDermid Iridite 14-2 hexavalent chrome conversion coating in accordance with The Boeing Company's internal process specification for IVD Al coating. All load bars were shipped to Omega Engineering, Justin, TX, with instructions to test each bar using the standard sustained load test as described in ASTM F519. The bars are loaded at 75\% of notch fracture strength (NFS) for 200 hours; specimens passing the static load test were then step loaded at increments equal to $5 \%$ NFS each hour to $100 \%$ NFS, or bar failure. Hydrogen embrittlement is normally evaluated on total time to failure. Since loading was performed at increments of NFS, an equivalent total time to failure was calculated by multiplying the percent of NFS by its corresponding load time. 


\subsubsection{Hydrogen Re-embrittlement}

Sixteen ASTM F 519 1a.1 load bar specimens and eight 1" $\times 4$ " $\times 0.040 " 4340$ steel specimens were IVD Al coated in Boeing production facilities and plasma coated at UMC. Sixteen load bars and eight steel panel specimens were shipped to Omega Engineering, Justin, TX, with instructions for re-embrittlement testing in each of the following solutions: distilled water, 3.5\% NaCl solution, Cyrotech SAE-AMD-1453 E36 Runway Deicing Fluid, 50\% solution and Penair HD-1 MIL-PRF-87937, Type I Aerospace Equipment Cleaning Compound, 10\% solution. Testing was performed per ASTM F1624 Incremental Step Loading Technique for hydrogen re-embrittlement. All load bar specimens were loaded to $45 \%$ NFS for 150 hours and the notch remained wet for the test period; specimens passing the static load test were then step loaded at increments equal to 5\% NFS each hour to $100 \%$ NFS, or until bar failure. An equivalent total load time was calculated in the same manner as stated in the previous section.

\subsubsection{Electrochemical measurements}

Four 4340 steel panels of $1 " \times 6$ " $\times 0.04$ " were obtained from The Boeing Company and IVD coated at UMC. Panels were viewed with a Scanning Electron Microscope (SEM) to confirm IVD microstructure. An EG\&G potentiostat/galvanostat (Model 273A) was used for anodic potentiodynamic polarization measurements. The potentiostat was controlled by EG\&G 352 SOFTCORR III corrosion measurement software. Measurements were carried out at room temperature in an aqueous salt solution $[0.5 \%$ sodium chloride $(\mathrm{NaCl})+0.35 \%$ ammonium sulfate $\left(\left(\mathrm{NH}_{4}\right)_{2} \mathrm{SO}_{4}\right]$. The working electrode was IVD coated steel panels with and without plasma nanocoating. The working area was $1 \times 1 \mathrm{in}^{2}$; the rest of the panel surface was masked with insulating tape. The counter electrode was a graphite rod $(0.6 \mathrm{~cm}$ 
in diameter), and the reference electrode was a standard silver-silver chloride $(\mathrm{Ag} / \mathrm{AgCl})$ reference electrode. All scans were taken over a twenty minute time period in the same salt solution. The scan rate for the anodic potentiodynamic polarization tests was $5 \mathrm{mV} / \mathrm{s}$. The test was terminated before passivation occurred.

\subsubsection{XPS Analysis}

X-ray photoelectron spectroscopy (XPS) data was acquired using a Kratos Axis 165 Photoelectron Spectrometer using an $\mathrm{Mg}$ anode at $225 \mathrm{~W}$. Survey and high resolution spectra were collected at pass energies of 80 and $20 \mathrm{eV}$ respectively, and a step size of 0.5 and $0.1 \mathrm{eV}$ respectively. CasaXPS V. 2.3.15 software was used to analyze the spectra.

\subsection{Results and Discussion}

\subsubsection{Hydrogen Embrittlement}

Table 3.1 shows the hydrogen embrittlement test results of plasma coated samples along with test controls of chromate conversion and bare IVD coated high-strength steel samples. Three out of six plasma nanocoated samples lasted to 95\% NFS loading. It can be see that, being similar to the test controls, TMS plasma nanocoating was not found to cause any hydrogen embrittlement. Table 3.2 shows the calculated total time to failure for hydrogen embrittlement of plasma coated specimens and the chromate conversion and bare IVD coated controls. It was noted that levels of loading and time to failure were similar to bare IVD and Iridite conversion coated IVD controls. An ANOVA single factor statistical analysis of the calculated total time to failure results in a P-value of 0.705 between samples, 
indicating there is no statistical difference between bare, conversion coated, and plasma nanocoated IVD for total time to failure.

TMS plasma coating was found to resist hydrogen re-embrittlement as shown in Table 3.3. It can be seen that 15 of the 16 plasma coated specimens passed the 150 hour hydrogen re-embrittlement test. Only the specimen \#18, in Table 3.3, failed at 13 hours into the 150 hour load test. The subject bar was retrieved and examined by scanning electron microscopy (SEM) to determine if failure occurred due to embrittlement or ductile failure. Figure 3.1 includes SEM images of the failure origin. In Figure 3.1 (d) \& (e), the fracture surface appears to indicate ductile failure, hence no hydrogen embrittlement; however the amount of corrosion present in the failure area weights conclusions regarding the failure mode more to the side of speculation. The subject bar was tested in salt water, so corrosion after failure was essentially immediate. 
Table 3.1. Hydrogen embrittlement test results for TMS plasma nanocoated, chromate conversion coated, and bare IVD Al coated high-strength steel specimens.

\begin{tabular}{|c|c|c|c|c|}
\hline Treatment & Sample \# & $\begin{array}{c}\text { Failed @ } 75 \% \\
\text { NFS - 200 hours } \\
\end{array}$ & $\begin{array}{c}\text { Time of Failure } \\
\text { @ } 75 \% \text { NFS }\end{array}$ & $\begin{array}{l}\text { Step Loading } \\
\text { Failure }\end{array}$ \\
\hline \multirow{6}{*}{$\begin{array}{l}\text { Plasma } \\
\text { Coating }\end{array}$} & 1 & No & N/A* & $\begin{array}{l}17 \text { minutes@ } \\
90 \% \text { NFS }\end{array}$ \\
\hline & 2 & No & N/A & $\begin{array}{l}\text { At step to } 95 \% \\
\text { NFS }\end{array}$ \\
\hline & 3 & No & N/A & $\begin{array}{l}20 \text { minutes @ } \\
95 \% \text { NFS }\end{array}$ \\
\hline & 4 & No & N/A & $\begin{array}{l}36 \text { minutes@ } \\
90 \% \text { NFS }\end{array}$ \\
\hline & 5 & No & N/A & $\begin{array}{l}\text { At step to } 95 \% \\
\text { NFS }\end{array}$ \\
\hline & 6 & No & N/A & $\begin{array}{l}4 \text { minutes @ } \\
90 \% \text { NFS }\end{array}$ \\
\hline \multirow{3}{*}{$\begin{array}{l}\text { Chromate } \\
\text { Conversion } \\
\text { Coating } \\
\text { (Control) }\end{array}$} & 7 & No & N/A & $\begin{array}{l}\text { At step to } 95 \% \\
\text { NF }\end{array}$ \\
\hline & 8 & No & N/A & $\begin{array}{l}47 \text { minutes @ } \\
90 \% \text { NFS }\end{array}$ \\
\hline & 9 & No & N/A & $\begin{array}{l}\text { At step to } 95 \% \\
\text { NFS }\end{array}$ \\
\hline \multirow{3}{*}{$\begin{array}{l}\text { Bare IVD } \\
\text { (Control) }\end{array}$} & 10 & No & N/A & $\begin{array}{l}39 \text { minutes @ } \\
90 \% \text { NFS }\end{array}$ \\
\hline & 11 & No & N/A & $\begin{array}{l}\text { At step to } 90 \% \\
\text { NFS }\end{array}$ \\
\hline & 12 & No & N/A & $\begin{array}{l}11 \text { minutes@ } \\
95 \% \text { NFS }\end{array}$ \\
\hline
\end{tabular}

*No failure was observed within the 200 hour test period in $75 \%$ NSF.

Table 3.2. Calculated total time to failure for hydrogen embrittlement of TMS plasma nanocoated, chromate conversion coated, and bare IVD Al coated high-strength steel specimens.

\begin{tabular}{lc}
\hline Treatment & Normalized Total Time to Failure (min) \\
\hline Plasma Coated & $9137.72+-26.32$ \\
Chromate conversion Coated & $9149.1+-6.75$ \\
Bare IVD & $9132.18+-32.27$ \\
\hline
\end{tabular}


Table 3.3. Hydrogen re-embrittlement test results for TMS plasma coated IVD Al highstrength steel specimens in various corrosive solutions.

\begin{tabular}{ccccc}
\hline Solution & Sample \# & $\begin{array}{c}\text { Failed @ 45\% NFS, } \\
\text { 150 hours }\end{array}$ & $\begin{array}{c}\text { Time of Failure @ } \\
\text { 45\% NFS }\end{array}$ & $\begin{array}{c}\text { Step Loading } \\
\text { Failure }\end{array}$ \\
\hline & 13 & No & N/A* & $\begin{array}{c}16 \text { minutes @ 95\% } \\
\text { NFS }\end{array}$ \\
& 14 & No & At step to 100\% \\
NFS
\end{tabular}

*No failure was observed within the 150 hour test period in $45 \%$ NSF. 

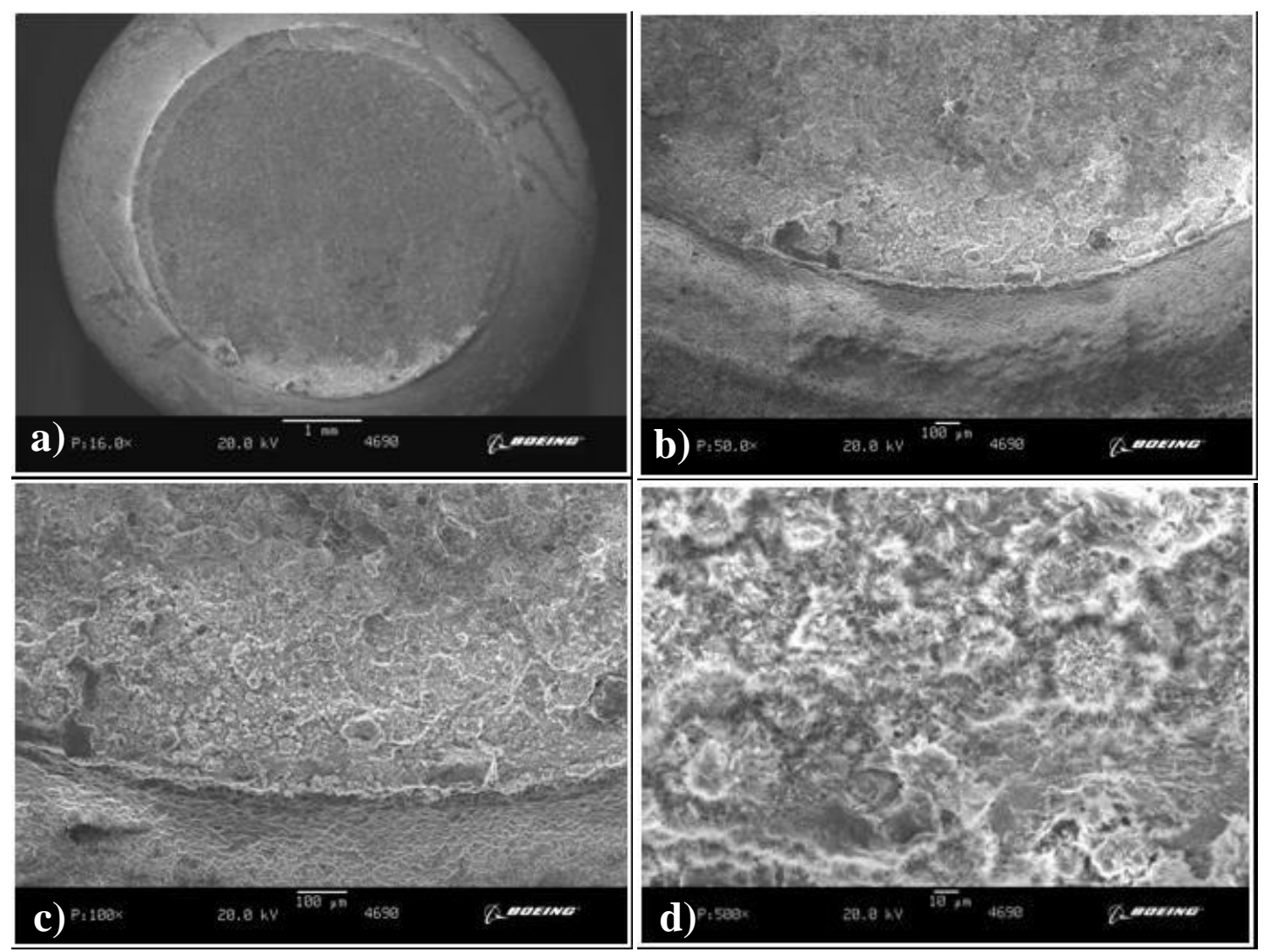

Figure 3.1. SEM Images of ASTM F 519 1a.1 Bar of specimen \#18 shown in Table 3.3: a) Load Bar End; b) Origin of Failure; c) Increased Magnification of Origin; d) Overload and Surface Oxidation near Origin.

In Table 3.3, the average normalized total time to failure for plasma coated IVD is $4442 \pm$ 74 minutes in distilled water and $4210 \pm 78$ minutes in $3.5 \% \mathrm{NaCl}$ solution (excluding sample \# 18). ASTM F1624 has been the aerospace industry hydrogen re-embrittlement testing standard since its release in 1977, and the Boeing Company already has a vast understanding of re-embrittlement of bare IVD Al coated high-strength steels. In this study, therefore, we did not perform testing on bare IVD controls for re-embrittlement because it is already known how the IVD Al coating performs. For examples, any coating applied to IVD Al that exhibits over 150 hours @ 45\% NFS while immersed in any of the embrittling solutions is considered a huge advancement as compared with bare IVD Al coated high-strength steels. Plasma coated IVD in E36 runway deicer and Penair HD 1 aircraft cleaner reached nearly 
$100 \%$ NFS during re-embrittlement testing. This is less embrittlement than what is caused by $3.5 \%$ salt solution, so runway deicer and Penair HD 1 can be considered safe to use with plasma coatings.

Iridite chromate conversion coatings require baking to release hydrogen resulting from the plating process. Removing the chromate coating greatly shortens the total amount of materials processing required, but it is also necessary since chromate is considered highly toxic. Chromate conversion coating and IVD Al are both passivation layers and do not completely stop corrosion, but instead corrode preferentially to the underlying steel. Contact of dissimilar metals causes an electrochemical potential which may actually increase the rate of corrosion and therefore the production of hydrogen. TMS plasma coating provides an advantage compared to passivation in two ways: 1) TMS plasma coating impedes penetration of corroding fluids which causes less hydrogen to be created, 2) TMS plasma coating impedes the transport of any hydrogen produced when some corroding fluids do inevitably reach the metal surface. Further, plasma coating adds few extra processing steps compared to the extensive processing incurred during electroplating of chromate coatings. Therefore, plasma coating is a good candidate for replacement of chromate conversion coating for corrosion protection.

\subsubsection{Corrosion protection}

Corrosion properties of the TMS plasma nanocoated specimens were measured using anodic potentiodynamic polarization. Figure 3.2 shows electrochemical polarization curves of untreated IVD and TMS plasma coated IVD. Anodic potentiodynamic polarization shows the corrosion behavior in the area of the critical anodic current density, which is the point where the current begins to increase rapidly as the applied potential increases. Corrosion 
potential and corrosion current were calculated by interpolating the plot at the sharp peak. The slopes of the electrochemical polarization curves are similar implying a similar capacitance of the passivating oxide layer [12]. Corrosion potentials $\left(\boldsymbol{E}_{\boldsymbol{C o r r}}\right)$ and corrosion currents $\left(\boldsymbol{I}_{\text {Corr }}\right)$ are summarized in Table 3.4. Plasma coated IVD shows a more positive corrosion potential and a lower current while bare IVD samples shows a more negative corrosion potential and higher corrosion current, thus TMS plasma nanocoatings improved corrosion resistance of IVD Al coated steel specimens.

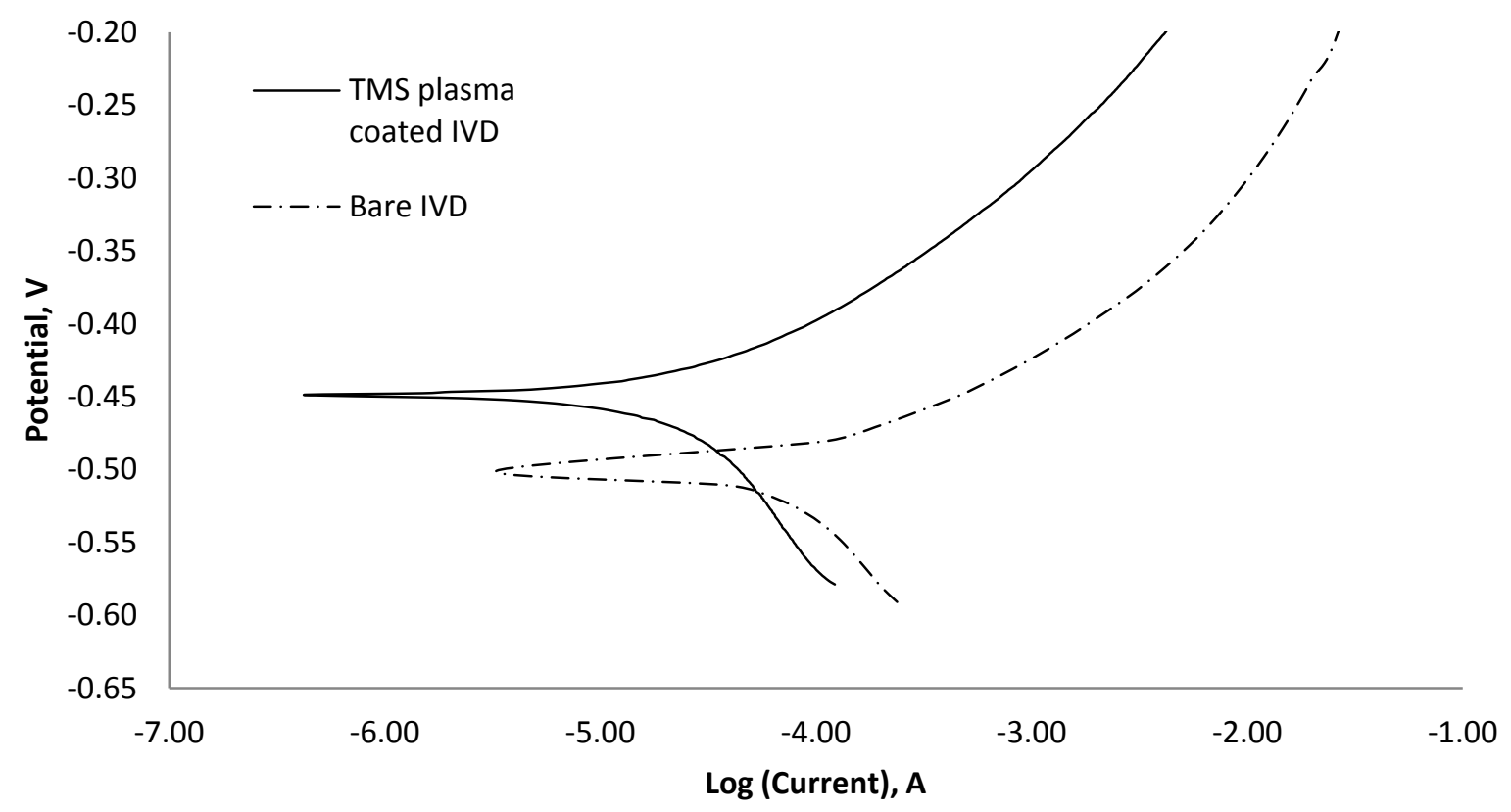

Figure 3.2. Typical anodic potentiodynamic polarization curves of TMS plasma coated and bare IVD Al coated steel specimens. 
Table 3.4. Electrochemical measurement results of TMS plasma coated and bare IVD Al coated steel specimens using electrochemical polarization.

\begin{tabular}{lll}
\hline Sample & $\boldsymbol{I}_{\text {corr }}(\mathbf{A})$ & $\boldsymbol{E}_{\text {corr }}(\mathbf{m V})$ \\
\hline TMS plasma coated IVD & $29.0 \pm 1.5 \times 10^{-6}$ & $-453.7 \pm 3.602$ \\
Bare IVD & $77.4 \pm 6.2 \times 10^{-6}$ & $-499.9 \pm 1.766$ \\
\hline
\end{tabular}

It should be pointed out that TMS plasma coatings were very thin used in this study and therefore provide very limited corrosion protection. This is evident by the less than one full log change in both $\boldsymbol{E}_{\text {corr }}$ and $\boldsymbol{I}_{\text {corr }}$ with plasma coatings as compared with the bare IVD controls. Therefore, TMS plasma coatings by themselves are not a sufficient barrier to corrosion. It is in the superior adhesion to the substrate and applied primer paints where TMS plasma coatings offer their greatest enhancement to corrosion resistance $[11,13,14]$. The samples tested in this study were not painted before embrittlement testing, yet the coating substantially decreased susceptibility to hydrogen re-embrittlment. The amount of hydrogen re-embrittlment is dependent on a number of factors: the amount of hydrogen formed by the reduction of water, the electrochemical potential of the coating, and the rate of transport of the hydrogen through the coating. Pure aluminum has a low hydrogen diffusion coefficient, but the open columnar structure of IVD Al coatings allows easy penetration of water solution that causes corrosion. TMS plasma coatings have a very hydrophobic surface that resists penetration of water solution to underlying steel substrates and slows down diffusion of corrosive solutions. As a result, application of plasma nanocoatings could protect the IVD specimens from corrosion and thus reduce the amount of hydrogen formed due to corrosion. The reduced amount of hydrogen formed by corrosion was considered as the major cause of decreased hydrogen re-embrittlement. 


\subsubsection{Plasma nanocoating characterization}

X-ray photoelectron spectroscopy (XPS) was performed to characterize the TMS plasma nanocoating and the typical XPS scan spectra are shown in Figure 3.3. As summarized in Table 3.5, it can be seen that the main chemical composition of the TMS plasma nanocoatings including $\mathrm{Si}, \mathrm{C}$, and oxygen. It is well known that tetramethylsilane [ $\left.\mathrm{Si}-\left(\mathrm{CH}_{3}\right)_{4}\right]$ plasma coatings consist mainly of a cross-linked $-\left[\mathrm{CH}_{3}\right]_{n} \mathrm{SiH}_{\mathrm{m}^{-}}$structure [15]. High resolution XPS peaks, shown in figures 3.4-3.6, give evidence of repeating units of the methylsilane groups. The TMS plasma nanocoating is composed of many chemical groups causing the peak broadening. Although no oxygen was flowed into the system, oxygen was present in the spectra. The oxygen present is most likely from exposure to oxygen in atmosphere after the plasma deposition. Oxygen is incorporated into the plasma coating creating a silicon-oxycarbide structure with various sub-oxides [Yu IVD-3]. 


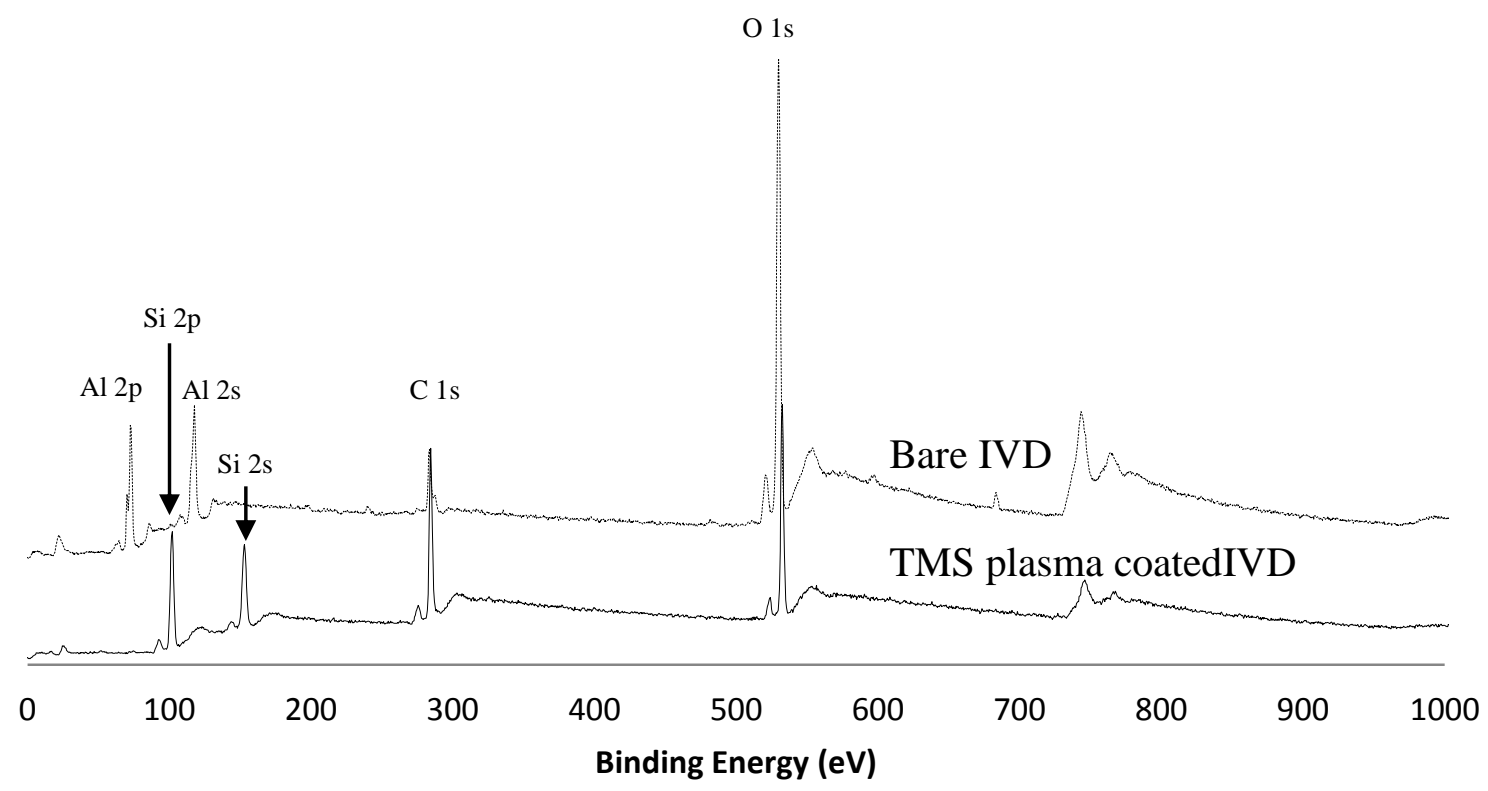

Figure 3.3. XPS scan spectra taken from the top surface of TMS plasma coated and bare IVD Al coated steels.

Table 3.5. Atomic concentration percentage of top surface of TMS plasma coated and bare IVD Al coated steels. obtained by XPS analysis.

\begin{tabular}{lllll}
\hline Sample & Al & O & C & Si \\
\hline TMS plasma nanocoating & 0.29 & 17.54 & 41.12 & 41.05 \\
Bare IVD & 48.5 & 33.74 & 13.69 & 4.07 \\
\hline
\end{tabular}




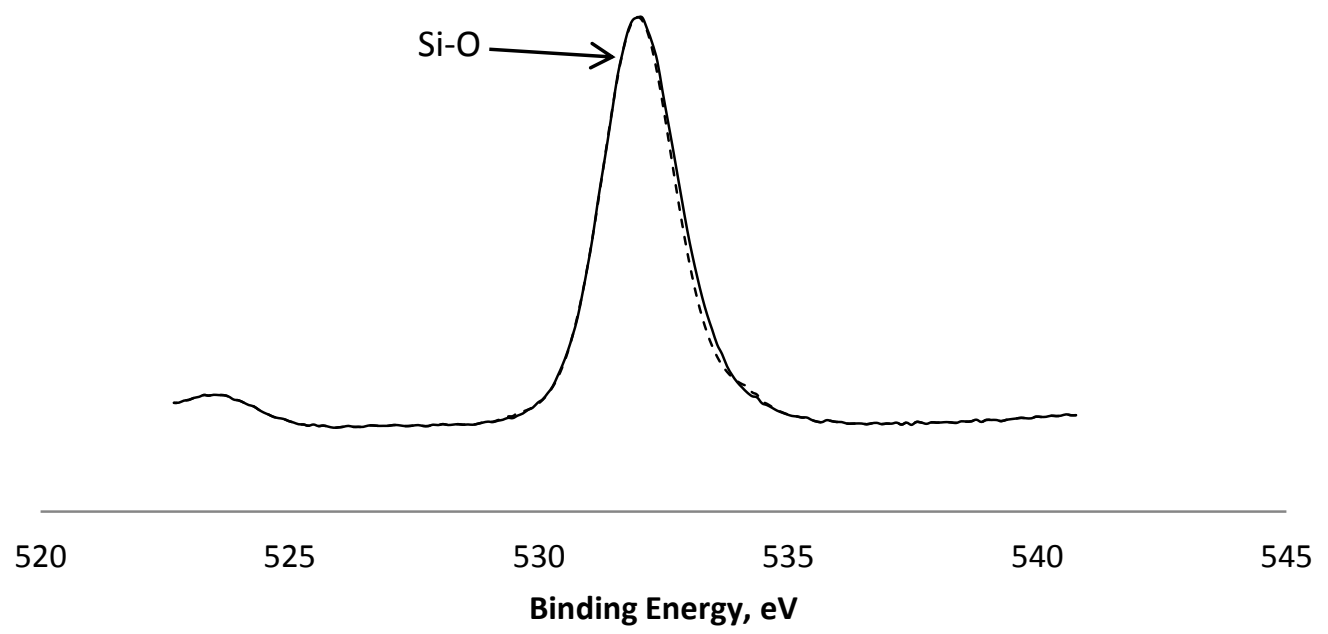

Figure 3.4. High resolution XPS spectra O1S peak taken from the top surface of TMS plasma nanocoating.

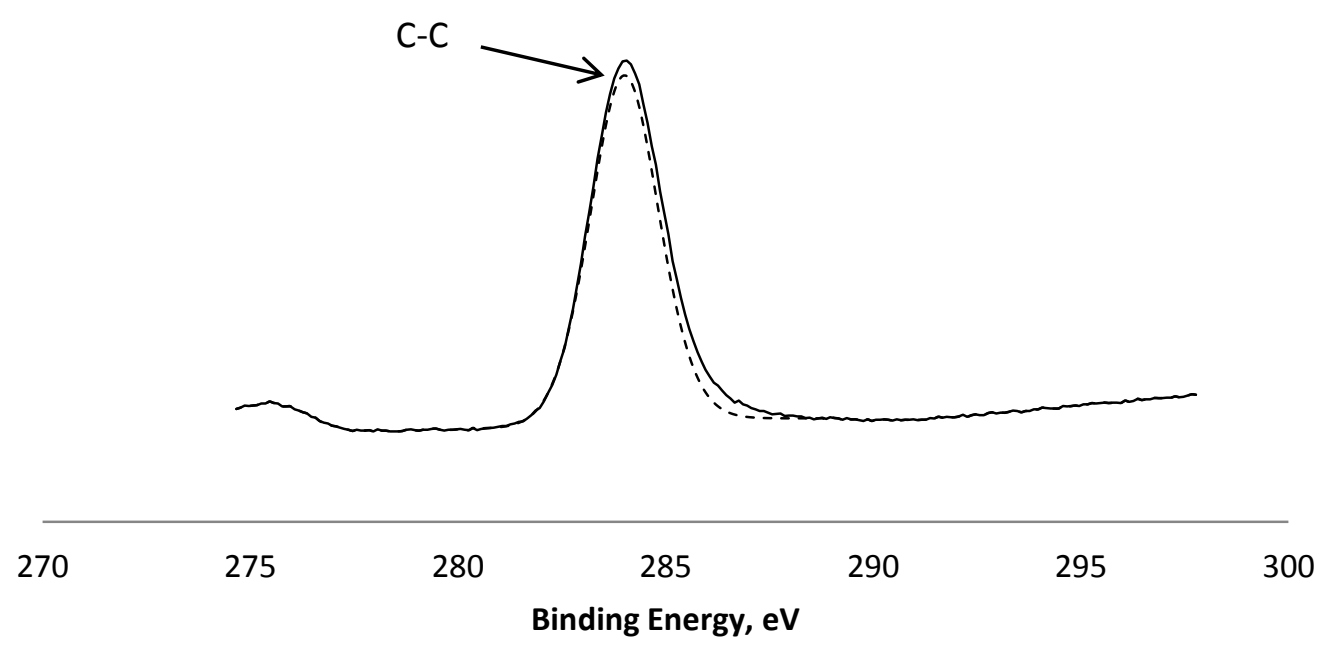

Figure 3.5. High resolution XPS spectra C1S peak taken from the top surface of TMS plasma nanocoating. 


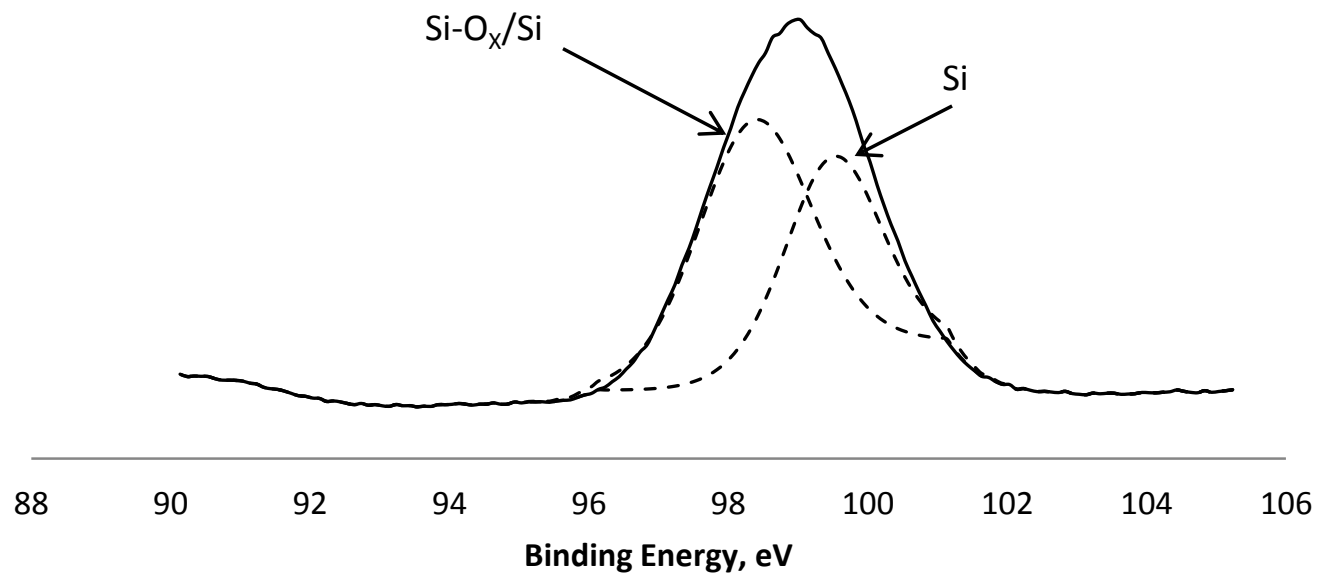

Figure 3.6. High resolution XPS spectra Si2P peak taken from the top surface of TMS plasma nanocoating.

\subsection{Conclusion}

TMS plasma nanocoatings were applied to IVD Al coated high-strength steel substrates. The process of applying TMS plasma nanocoatings to IVD coated steel was not found to cause hydrogen embrittlement to the steel substrates. The electrochemical measurement results indicated that the TMS plasma nanocoatings, which have a tight network of covalent bonds and hydrophobic surfaces, provided certain protection the IVD Al coated steel substrate from corrosion in various corrosive fluids and thus reduced amount of the corrosion byproducts that could cause hydrogen re-embrittlement. Re-embrittlement data generated and presented in this study demonstrates that following IVD Al coating with the plasma polymer coating can protect IVD coated steel from re-embrittlement. TMS plasma coated IVD Al coated steel specimens passed the 150 hours @ 45\% NFS, therefore it is considered a "passing" coating without any further treatments. Iridite chromate conversion coatings require baking to release hydrogen resulting from the plating process. In 
comparison with the hazardous chromate conversion coatings, TMS plasma coating provides an advantage compared to passivation in two ways: 1) TMS impedes penetration of corroding fluids which causes less hydrogen to be created, 2) TMS plasma coating impedes the transport of any hydrogen produced when some corroding fluids do inevitably reach the metal surface. In conclusion, plasma coating is a good candidate for replacement of chromate conversion coating for protection of IVD Al coated high-strength steels from corrosion and consequently from hydrogen re-embrittlement.

\section{Acknowledgements}

This study was supported by Battelle under contract number of W911NF-07-D-0001. The authors would thank Mr. Charles R. Valley, Mr. Brian R. Tobin, and Dr. Peter A. Lurker from Wright-Patterson AFB for overseeing the project. The authors would express their appreciation to Mr. Vernon Holmes from The Boeing Company, Mr. Mike Bridger, the president of MEB CO Services Inc. for their insightful suggestion on IVD Al coatings. The authors would also thank Dr. Andy Ritts and Dr. Young Jo Kim for performing some of the experiment work of this study. 


\subsection{References}

1. Findlay, S.J., Harrison, N.D., 2002, "Why Aircraft Fail," Materials Today, 5, 18-25.

2. Chalaftris, 2003, Evaluation of Aluminium-Based Coatings for Cadmium Replacement, (Doctoral Dissertation). Cranfield University, retriveved from dspace.lib.cranfield.ac.uk.

3. Courtney, T.H., 2000, Mechanical Behavior of Materials, Waveland Press Inc., Long Grove, Il., 630-679.

4. Figueroa, D., Robinson, M.J., 2008, "The effects of sacrificial coatings on hydrogen embrittlement and re-embrittlement of ultra high strength steels," Corrosion Science, 50, 1066-1079.

5. EPA Federal Register, National Emission Standards for Hazardous Air Pollutants for Source Categories: Aerospace Manufacturing and Rework Facilities, Vol. 60 (170), September 1995, p. 45947.

6. Pollock, W.J., 1998, "Hydrogen embrittlement of plated high-strength 4340 steel by galvanic corrosion," Galvanic Corrosion, 35-50.

7. Yasuda, H.K., Yu, Q.S., Reddy, C.M., Moffitt, C.E., Wieliczka, D.M., Deffeyes, J.E., 2001, "Barrier-adhesion principal for corrosion protection," Corrosion, 57, 670-679.

8. Yu, Q., Moffitt, C.E., Wieliczka, D.M., Deffeyes, J., Yasuda, H., 2002, “Corrosion protection of ion vapor deposition (IVD) Al-coated $\mathrm{Al}$ alloys by low-temperature plasma interface engineering Part III - DC cathodic polymerization in a closed reactor system," Organic Coatings, 44, 37-47.

9. Grundmeier, G., Thiemann, P., Carpentier, J., Barranco, V., 2003, "Tailored thin plasma polymers for the corrosion protection of metals," Surface and Coatings Technology, 174-175, 996-1001.

10. Chan, Y., Qingsong, Y., 2005, "Electrochemical characterization of plasma polymer coatings in corrosion protection of aluminum alloys," J. Vac. Sci. Technol. A, 23, 991-997.

11. Reddy, C.M., et al., 2000, "Improved corrosion protection of aluminum alloys by system approach interface engineering: part 1-Alclad 2024-T3," Corrosion, 56, 819831.

12. Kuznetsova, A., Yates, J.T., 2001, "Making a Superior Oxide Corrosion Passivation Layer on Aluminum Using Ozone," Langmuir, 17, 2146-2152. 
13. Yasuda, H., Yu, Q., Reddy, C., Moffitt, C., Wieliczka, D., 2001, “Adhesion of plasma polymer of trimethylsilane to aluminum alloys," Journal of Applied Polymer Science, 85, 1387-1398.

14. Yasuda, H., Yu, Q., Reddy, C., Moffitt, C., Wieliczka, D., 2002, “Adhesion of spray primers to plasma polymer coatings," Journal of Applied Polymer Science, 85, 14431457.

15. Fonseca, J., Apperley, D., Badyal, J., 1993, "Plasma polymerization of tetramethylsilane," Chemistry of Materials, 5, 1676-1682. 


\title{
Chapter 4 Construction of an Ion Vapor Deposition Plasma
}

\section{Nanocoating Hybrid Reactor}

\begin{abstract}
The construction of a lab scale ion vapor deposition (IVD) chamber is described. The chamber has been modified for deposition of organosilane plasma coating on top of IVD. IVD coating required electrical insulation of the substrate holder, specifically such that aluminum vapor did not condense on insulators causing electrical conductivity. IVD coating required specific coating time and coating rate. High aluminum vaporization rate resulted in loss of plasma and resulted in physical vapor deposition. IVD coating required cycling between IVD coating and 30 minutes of cooling for $100 \%$ IVD. Overheating of the substrate resulted in a large crystal physical vapor type structure. Plasma nanocoatings are applied with thicknesses depending on coating time and surface area of the substrate. Plasma nanocoatings were produced without contaminating the subsequent IVD coated panels.
\end{abstract}

\subsection{Introduction}

Thin layers of low porosity aluminum may be applied to metal surfaces using the technique known as ion vapor deposition (IVD). The high vacuum reactor chamber required for the IVD coating system lends itself well for plasma coating which also requires vacuum. Plasma coating, sometimes referred to as plasma polymerization, is typically applied in vacuum between two parallel plates, or barrel type reactors. There are various sources for power input: DC, pulsed DC, microwave, AC (kilohertz- Megahertz), induction, capacitive 
microwave, electron beam, laser induced, and a coupling of any of these variations [1,2]. Both IVD and plasma use a sample held at a high voltage. IVD also uses DC power for producing cathodic plasma around the substrate and similar wattage is used for both processes, therefore much of the same equipment can be used for both processes. In this case, the reactor chamber serves as the ground electrode. Since the cathodic region of the plasma exists near the substrate, the ground electrode may be far away and not parallel to the substrate and still develop a uniform coating.

IVD or Ion Plating is similar to the physical vapor deposition (PVD) method, i.e. evaporative deposition, commonly used in applying thin coatings of pure aluminum for corrosion resistance [3]. In PVD, a crucible is resistively heated and aluminum wire is fed into the crucible until vaporized. Vaporization of aluminum is performed under a vacuum to reduce contamination from elements in atmosphere and to lower the aluminum's vaporization temperature. IVD is the same as PVD except the substrate is held at a high negative potential $(\sim 1 \mathrm{kV})$ in an inert gas, producing a glow discharge. The glow discharge positively ionizes some of the vaporized aluminum. For low electron density, penning ionization is the dominating process for metal ionization, whereas for high electron density $\left(>>10^{17} \mathrm{~m}^{-3}\right)$ the metal ions are generated by electron impact ionization [4].The ionized aluminum is subsequently attracted and accelerated toward the cathode (substrate). The aluminum condenses on the substrate forming a thin shell of aluminum. For corrosion protection, desired thicknesses vary between 0.0003 to 0.001 inches.

The degree of ionization for many vacuum plasmas is very low, $0.1 \%-2 \%$ [5]. The majority of deposited material is therefore neutral and not ionized. IVD takes place in a vacuum around the micron range and aluminum vapor may collide multiple times with the 
inert gas. This will lower the energy of the vapor but it will also scatter the vapor allowing more coverage of substrate surfaces, therefore IVD coatings are considered uniform and not confined to line-of-sight coating. The inert background gas may also cause agglomeration of the vapor atoms. If allowed to coat the surface, these agglomerates can result in a nonadherent granular film. This problem is overcome in IVD because the particles become negatively charged when agglomerating and are therefore repelled by the cathode (substrate).

IVD coating can provide good adhesion between a film and surface [6]. The advantages of IVD with respect to adhesion are:

i) sputter cleaning contaminant particles from the substrate surface up to and during the time of film formation;

ii) energy of the surface atoms are increased making them mobile and able to align with the atoms in the substrate's crystal lattices with minimal bulk heating;

iii) atomistic addition of $\mathrm{Al}$ gives the best surface contact, filling in small pores and microcracks;

iv) implantation of aluminum atoms into the substrate causes mixing of the atoms in the interfacial region.

Adhesion is dependent on bonding across the interfacial region, type of interfacial region, and the fracture mechanism during failure. For the case of aluminum coated on steel, a diffused interface is beneficial because a gradient of mixed atoms distributes stress gradually over a greater area than if it were on one plane of contact. In IVD with a $1 \mathrm{kV}$ electric field, a $1^{+}$charged metal ion can be accelerated enough to penetrate $\sim 5 \mathrm{~nm}$ into the 
substrate and can penetrate further through diffusion [4]. A diffuse interface is important for adhesion especially for metals with much different coefficients of thermal expansion. When the two metals come into contact at elevated temperatures, stresses at the interface are low. As the metals cool, they contract at different rates but are bound together at the interface and stresses increase. This added stress acts as a preload and can lower the adhesive strength when further stresses are added. A gradient of mixed atoms results in a gradual difference in thermal expansion coefficients and therefore does not concentrate the stresses. If failure occurs, it is kept locally since crack propagation is minimized by the numerous boundaries.

A popular IVD coater used in industry, known as the Ividizer, is the coater that this research is trying to simulate with a small chamber similar to a bell jar reactor. The Ividizer is a $10^{\prime}$ long by 6' diameter cylindrical chamber with four crucibles for melting aluminum. The crucibles traverse the length of the Ividizer at around $1 / 2$ " per second giving off an aluminum vapor. The crucibles make multiple passes dependant on the area of coverage and desired coating thickness. The parts to be coated span the length of the chamber and are kept in a glow discharge the entirety of the coating process. In our research, we have seen that aluminum vapor can quench plasma in local areas without termination of the plasma as a whole. When coating with the Ividizer, it is possible that the aluminum vapor could be quenching the plasma local to the aluminum vapor while the plasma not experiencing the vapor remains. Once the vapor has moved to another location, the plasma resumes, possibly etching deposited aluminum back into the plasma which may then be ionized again and redeposited back onto the panel [7]. Further, alternating deposition and removal may cause the close-packed microstructure typically found in conventional IVD. Schiller et al. found alternating the substrate between ion bombardment from an inert gas and non-ionized 
aluminum vapor can produce the same microstructure to that found in conventional IVD [8].

In this case, the ion bombardment activates the substrate, giving surface atoms energy to fill voids and lattice points.

Atoms that do not penetrate into the substrate may condense onto the substrate surface. Condensation occurs in three steps: incident atoms transfer kinetic energy to the substrate lattice and become loosely bonded adatoms; the adatoms diffuse over the surface exchanging energy with the lattice or other adsorbed atoms; the atoms settle to become incorporated into the lattice by bulk diffusion processes [7]. Thonton uses zone models to predict coating microstructure based on the inert gas pressure and the ratio of substrate temperature, $\mathrm{T}$, to melting point temperature, $\mathrm{T}_{\mathrm{m}}$, of the coating material. PVD found in Zone $1\left(\mathrm{~T} / \mathrm{T}_{\mathrm{m}}<0.3\right)$ consists of tapered crystals that suffer from an open and columnar structure, as seen in Figure 4.1, making them susceptible to corrosion. Columns form due to a simple geometric effect termed shadowing $[4,7,9,10]$. Shadowing occurs due to high points caused by either the inherent roughness of the substrate surface or from nucleation of adatoms. Incident atoms with oblique trajectories collide with the high points causing them to receive more coating flux than valleys which continues to build these points higher. Atoms collect on the columns and relax to orient themselves with the column's crystal lattice (epitaxy). Zone 2 $\left(0.3<\mathrm{T} / \mathrm{T}_{\mathrm{m}}<0.5\right)$ consists of columnar grains separated by distinct intercrystalline boundaries. The difference between the zones is based on which growth factor is dominant, i.e. bulk diffusion, surface diffusion or shadowing. Thornton describes IVD to be similar to PVD found in Zone $\mathrm{T}\left(\mathrm{T} / \mathrm{T}_{\mathrm{m}} \sim 0.3\right)$, the transition zone between Zone 1 and Zone 2, at low $\mathrm{T} / \mathrm{T}_{\mathrm{m}}$. The similarity to Zone $\mathrm{T}$ is because ion bombardment can suppress the open Zone 1 structures by creating nucleation sites for arriving atoms, or by eroding surface roughnes 
peaks and redistributing material into valleys. It is also noted that the difference in microstructure between Zone 1 and Zone 2 is due to adatom mobility and therefore greater diffusion. High energy particles, as found in plasmas, will also add to the adatom mobility and therefore could be the cause of the Zone T like microstructure.

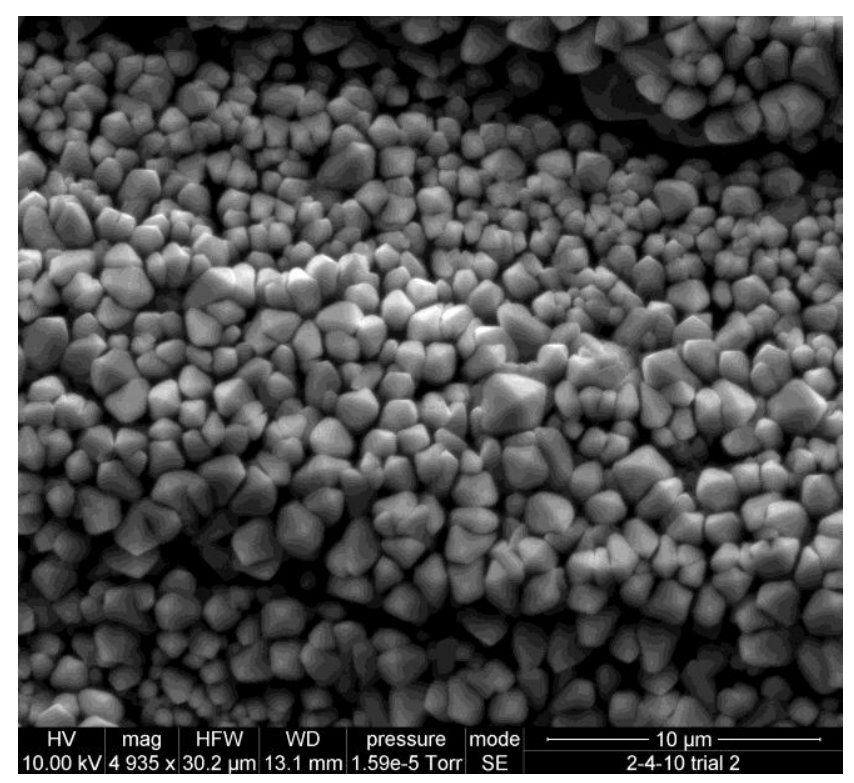

Figure 4.1. SEM of physical vapor deposited aluminum.

\subsection{Reactor Construction}

Construction of the IVD coating system consisted of construction of a vacuum system, fabrication of boat clamps to hold the heating boat, fabrication of a shutter, revision of a wire feeder, fabrication of a sample hanger, and installation of cooling systems for the boat holder and diffusion pump.

\subsubsection{Construction of the Vacuum System}

Modification of an IVD coater for plasma coating simply requires installation of an extra gas feed line for the TMS monomer including a mass flow controller. Using a 
mechanical and booster pump, the vacuum system is capable of pressures as low as .3 mTorr. IVD requires $<0.08$ mTorr base pressure, so a diffusion pump was added to the system as shown in Figure 4.2 and Figure 4.3. Using the diffusion pump, the vacuum successfully went to $\sim 0.02$ mTorr. All vacuum lines were leak tested by spraying acetone or isopropanol. The chamber leaks $\sim 0.08 \mathrm{sccm}$. Since argon is flowed in at $1 \mathrm{sccm}$, contamination is less than 1 molar\%.

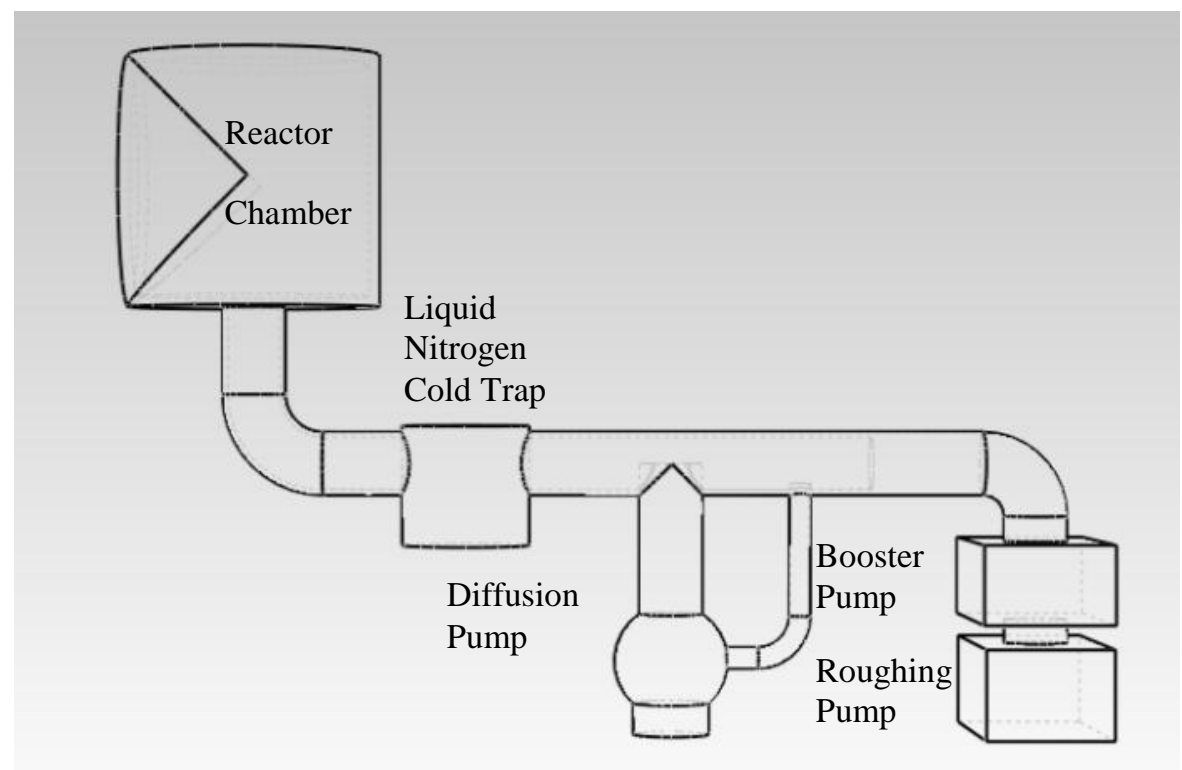

Figure 4.2. A depiction of the IVD/Plasma reactor vacuum system. 


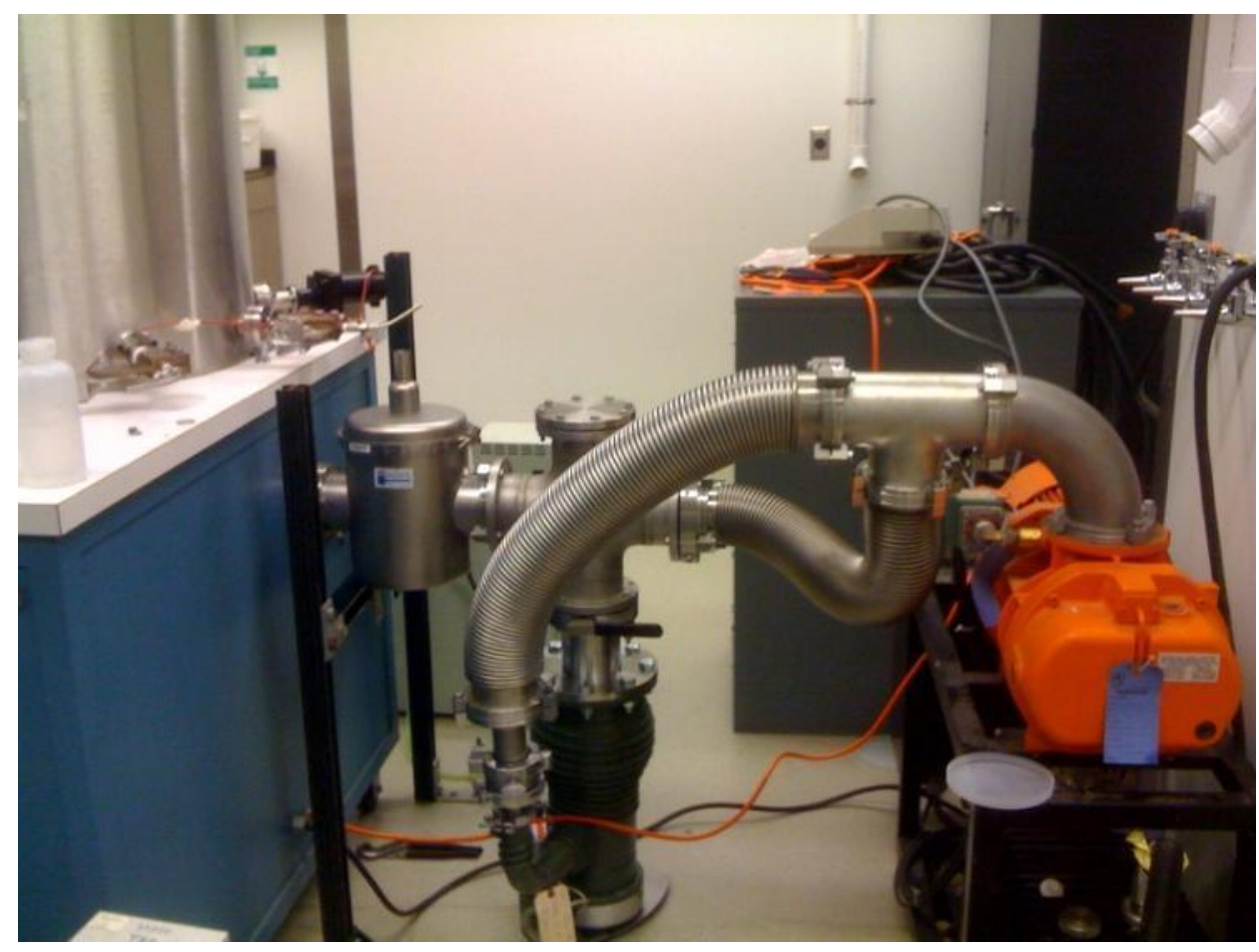

Figure 4.3. Photo of the assembled vacuum system.

\subsubsection{Modification of Boat Clamps}

Multiple designs were developed setting the clamps at a 45 degree angle with respect to each other as seen in Figure 4.4. These designs required the wire feeder nozzle to be placed at a high angle with respect to the boat. The high angle resulted in constriction of the wire and clogging. A third design was made, as shown in Figure 4.5, resulting in a lower angle between the wire feeder and the boat. This design was chosen and fabricated as shown in Figure 4.6. The only part needed was one sheet of 1/4" 110 copper alloy. 

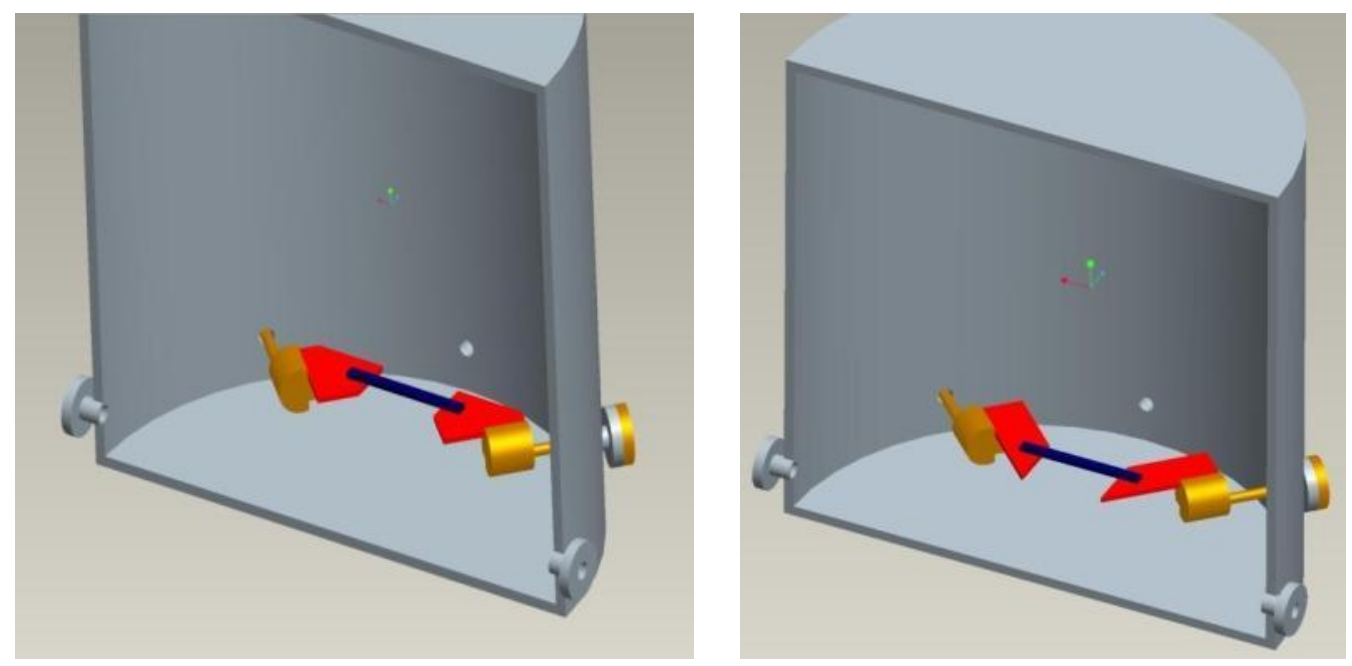

Figure 4.4. 3D depictions of the reactor chamber and boat holder with the proposed boat clamps in red and the estimated boat location in blue; two options for the clamp design.

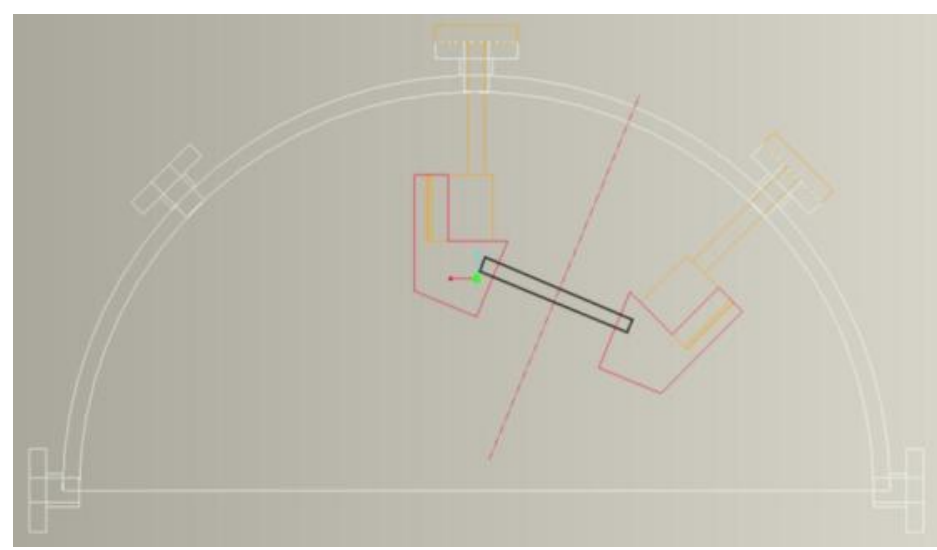

Figure 4.5. A $2 \mathrm{D}$ depiction of the final boat clamp design (blue) shown in relation to the reactor; dimensions are inches.

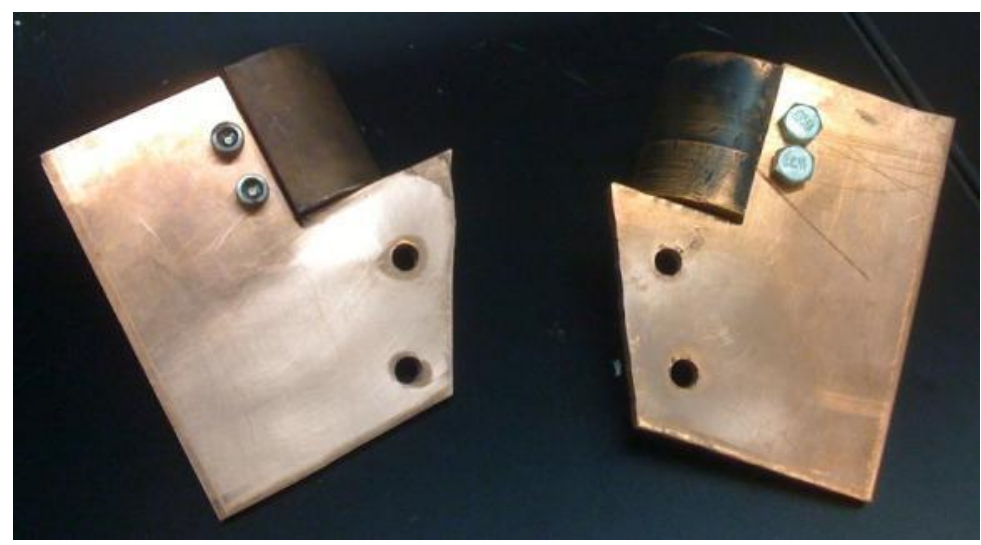

Figure 4.6. Photo of new boat clamps for the larger reactor. 


\subsubsection{Shutter Design and Construction}

The basic shutter design can be seen in Figure 4.7. The top plate was suspended from an axle which is allowed to rotate by use of a knob on top of the reactor while the bottom plate remains stationary. The openings in the plates were offset to close the shutter to build up a homogeneous aluminum vapor. Once the vapor has accumulated, the plate openings will are aligned to allow the vapor to enter the rest of the chamber and to deposit on the sample. The top plate was made to have two openings to reduce weight on the suspension axle and it was made symmetrical to reduce stress on the axle. It was found that the bottom plate was not needed in order to achieve a homogeneous coating and was therefore not used.
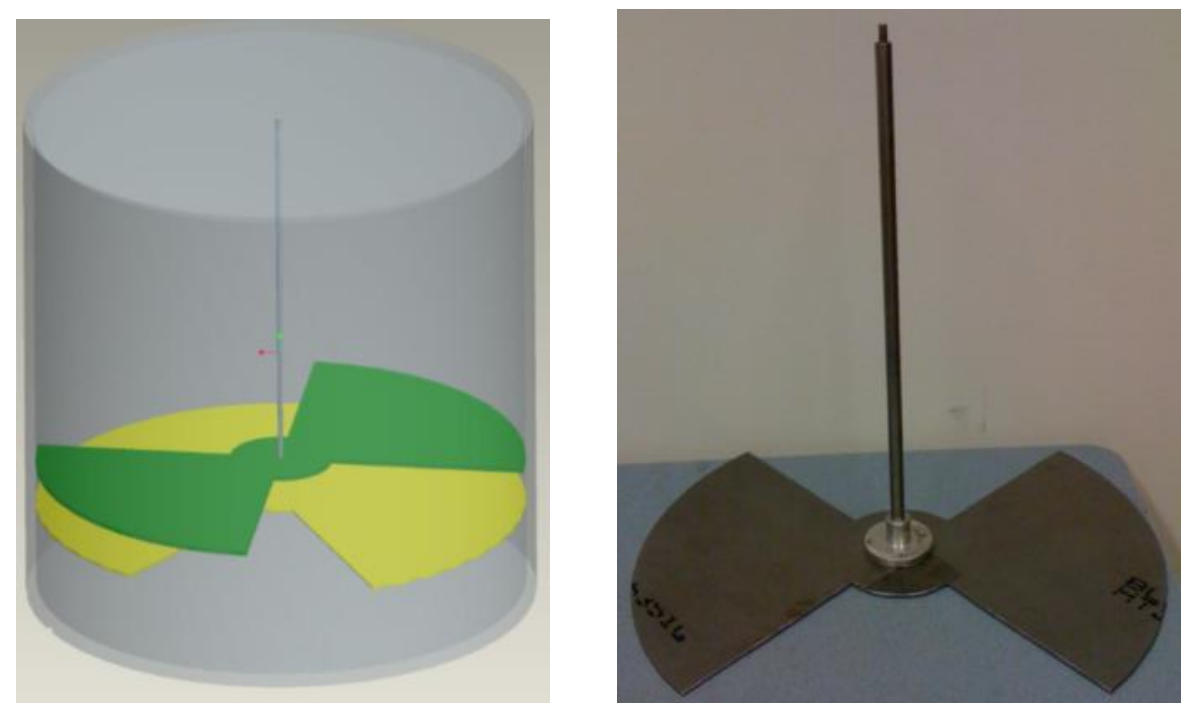

Figure 4.7. A depiction of the basic shutter design (left), a photo of the constructed shutter (right). 


\subsubsection{Wire Feeder Modification}

A plate was added to the bottom of the wire feeder to add stability as seen in Figure 4.8. The wire feeder was insulated by using an alumina or polymer rod for the drive shaft and a piece of Teflon under the base.

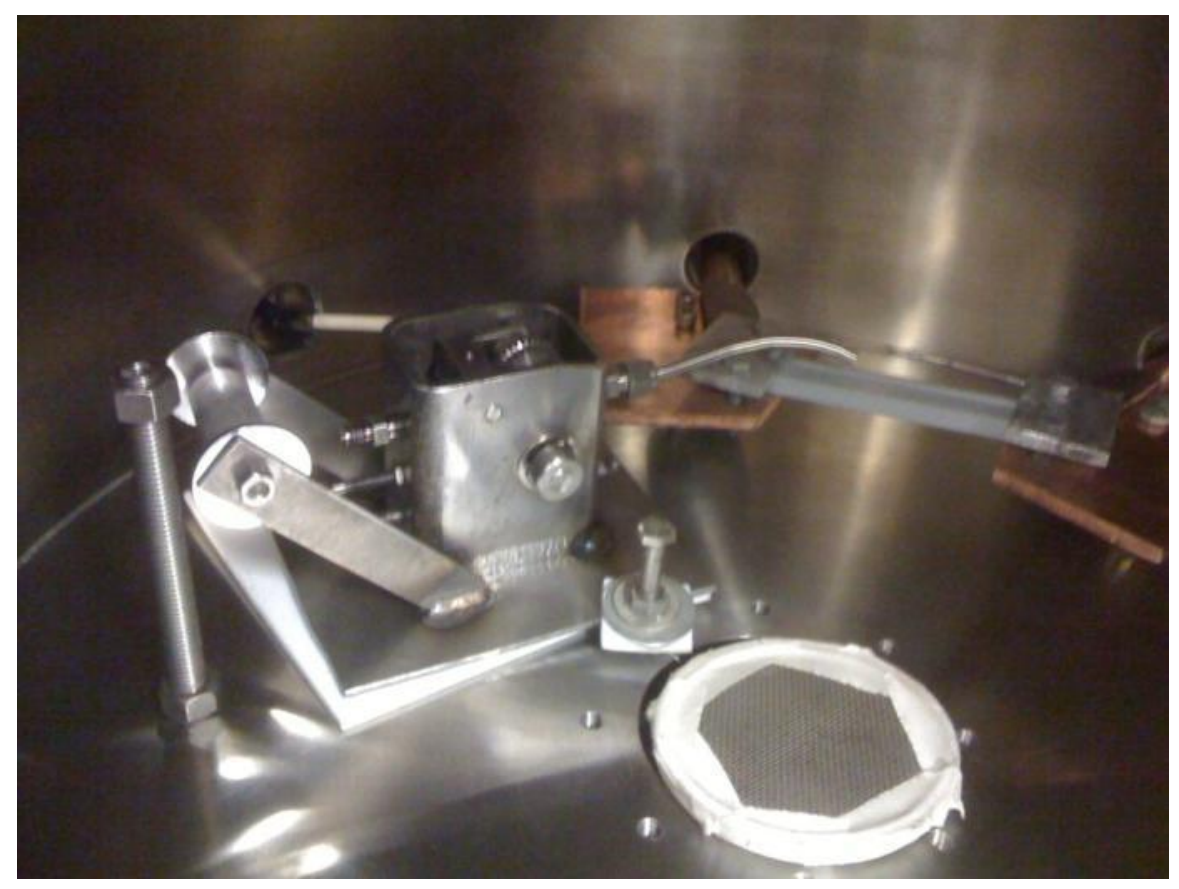

Figure 4.8. Photo of wire feeder and heating boat.

\subsubsection{Installation of Pressure Controller, Baratrons, and Mass Flow Controllers}

Gas lines were connected to the mass flow controllers and to the reactor along with a variable pressure controller. The gas lines were leak tested the same as the vacuum lines. Stopping leaks at valves was considerably challenging; Teflon tape was often used for better seals. Two baratrons (digital barometers) were used in this experiment. The first baratron was used for measuring the working pressure (6-50 mTorr) since it is accurate down to 1 mTorr while the second baratron was used as an input for the pressure controller and to measure the base pressure $(<0.08$ mTorr $)$ since it was accurate down to 0.025 mTorr. 


\subsubsection{Installation of the Cooling System}

Two cooling pumps were used to keep the diffusion pump and heating boat clamps at safe operating temperatures. The pumps ran at their maximum flow rate. The first cooling system on the diffusion pump was not capable of keeping the diffusion pump at a sufficiently low temperature; cooling lines were near melting. A larger cooling system was used and the diffusion pump can now be used for over an hour before unsafe heating. The diffusion pump temperature rose steadily so the refrigeration temperature was lowered to near $0^{\circ} \mathrm{C}$ before use to allow the longest amount of safe operation time.

\subsubsection{Installation of the Heating Boat Power Supply}

The heating boat was powered by a high amperage power supply. The original electrical lugs connecting the power lines to the heating boat clamps were too small for the power cords so larger lugs were used as shown in Figure 4.9. Two plastic storage containers were modified to shield against anyone touching the clamps during operation. The boat was successfully heated as shown in Figure 4.10. 


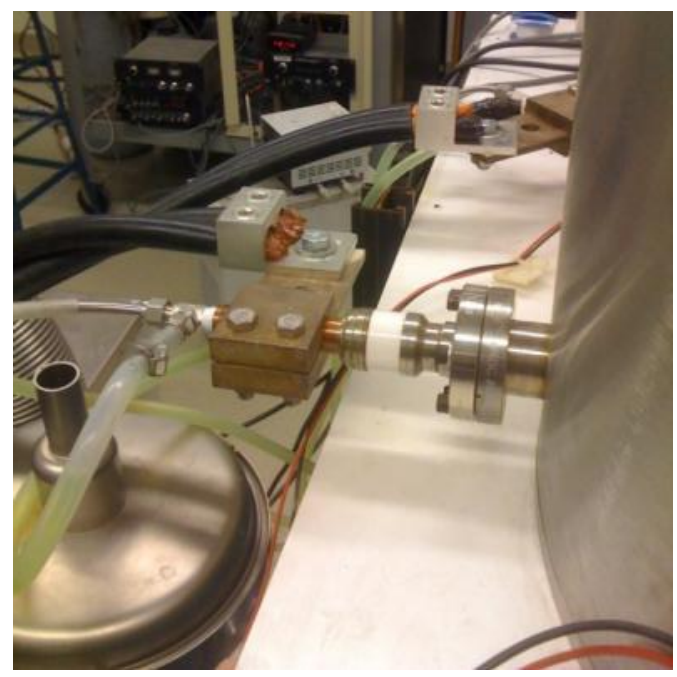

Figure 4.9. Photo of larger lugs for powering the heating boat.

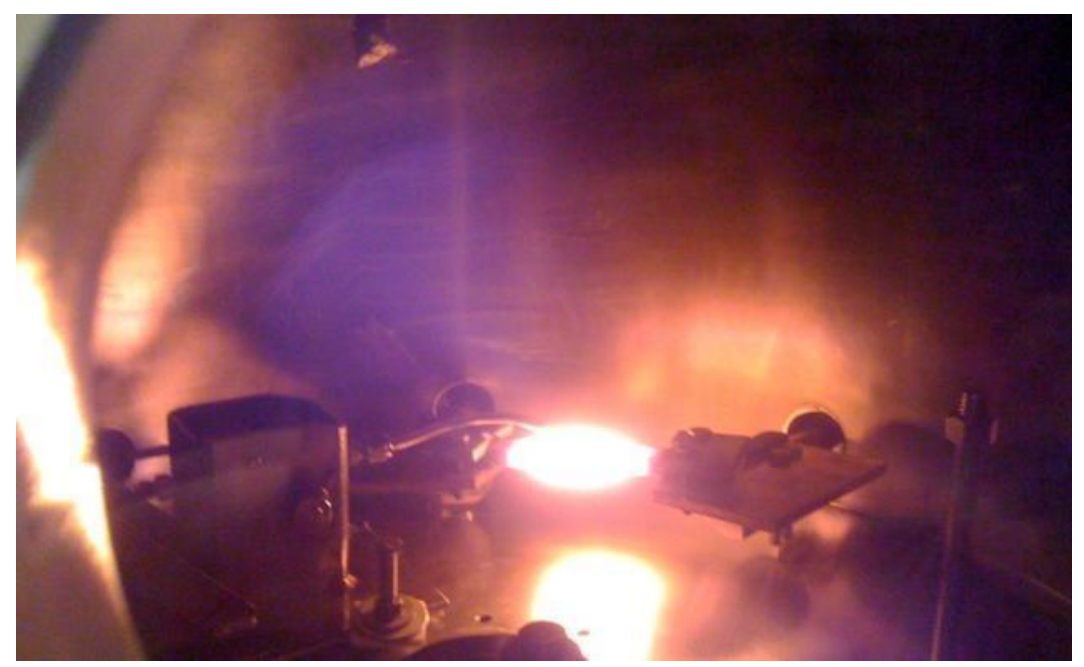

Figure 4.10. Photo of heating of the heating boat.

\subsubsection{Shielding the Inside of the Reactor}

A small percentage of vaporized aluminum does not condense on the sample substrate and coats the reactor walls and other parts in the reactor. Since we are aiming for great adhesion, the overspray is hard to remove; therefore the inside of the reactor was covered with aluminum sheet metal and aluminum foil. 


\subsubsection{IVD Operating Procedure}

A procedure to coat panels with IVD was developed:

1. Put 1 " of aluminum wire in the heating boat to avoid crucible dry-out.

2. Pump down reactor to base pressure $(<0.08 \mathrm{mTorr})$.

3. Flow argon $1 \mathrm{sccm}$ at $6 \mathrm{mTorr}$.

4. Plasma cleaning until determined clean (no sparks) at .5A, any voltage.

5. Ensure shutter is closed and boat cooling is on (near $0{ }^{\circ} \mathrm{C}$ ).

6. Warm up boats to $250 \mathrm{~A}$ at $250 \mathrm{~A} / 3 \mathrm{~min}$, hold at $250 \mathrm{~A}$ for $2 \mathrm{~min}$.

7. Ramp to $\sim 500 \mathrm{~A}$ over 1 minute.

8. Feed wire at $27 \mathrm{in} / \mathrm{min}$.

9. When wire is vaporizing properly, open the shutter, start plasma at .5A, any voltage.

10. Coat for a determined amount of time depending on class of coating.

11. Close shutter, turn off plasma, turn off heating boat.

12. Pump down to base pressure to remove residual IVD vapor.

\subsubsection{Troubleshooting Plasma Quenching During IVD}

It was confirmed on multiple trials that the electrode setup was a location of failure. It is believed that vaporized aluminum condensed on the insulated wires holding up the panel. Once coated, the surface of the insulators became conductive and a short circuit was made causing the plasma to go out. The different cathode electrode connections can be seen in figures 4.11-4.14. Cathodes should be insulated and shielded so no IVD is allowed to coat the insulator [6]. One method of holding the panel with insulators instead of the metal wires resulted in a plasma surrounding the entirety of the panel, but also caused instabilities of the plasma. It is believed that as the panel is charged, the insulators build up a surface charge until its potential becomes high enough to arc. This releases the charge on the panel making the plasma unstable. Insulators were placed in glass tubes such that the depth to the insulator was at least twice that of the diameter of the tube. A stable plasma was thus achieved. Although a stable plasma was achieved during the aluminum vaporization stage, the plasma seemed confined to the top of the panel. Wires above the panel, used to hold the panel in 
position, were the cause of the confined plasma. This is because electric fields are stronger along the thin wires due to their small size. The resulting coating was PVD as confirmed by SEM shown in Figure 4.15. A new electrode setup, shown in Figure 4.16, was designed such that it insulated the panel near the panel effectively confining the plasma to the panel.

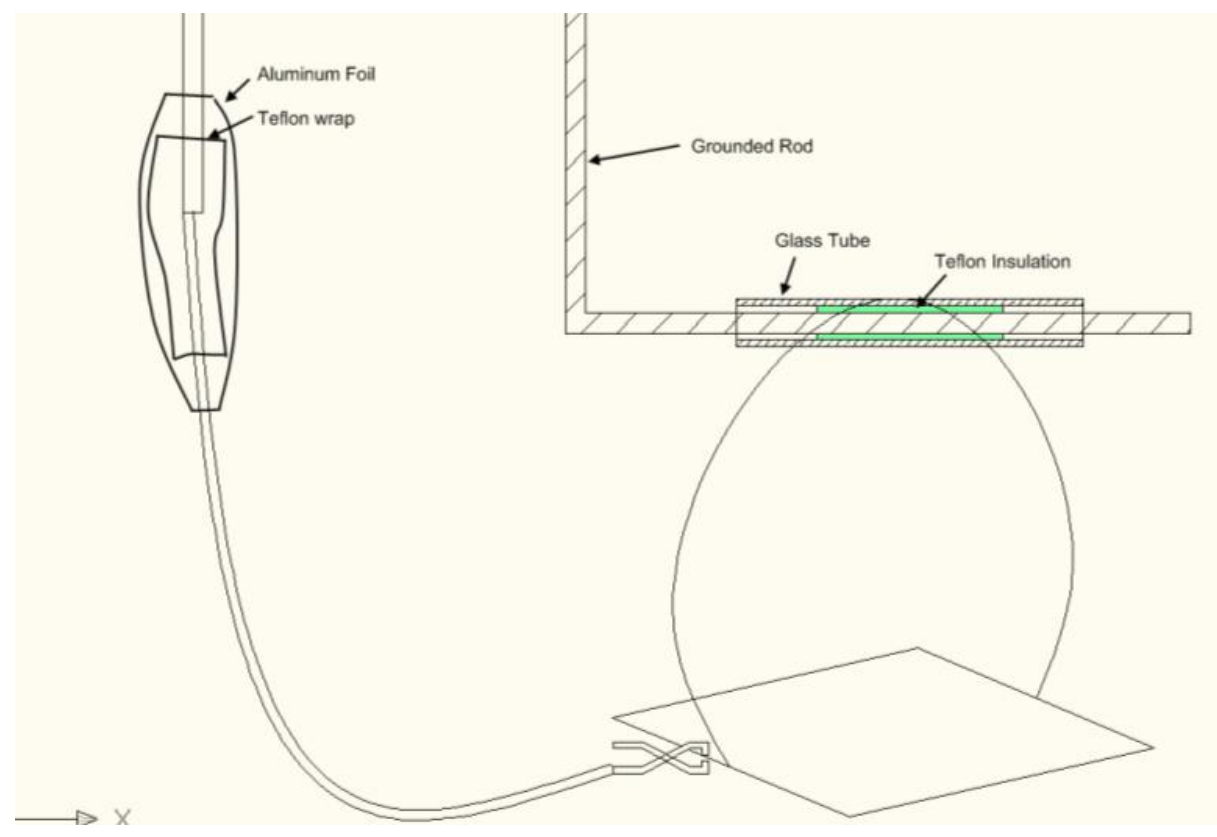

Figure 4.11. A depiction of the electrode setup where the wire holding the panel up sets on a glass tube that is insulated from the rod; IVD cannot penetrate into the glass tube. 


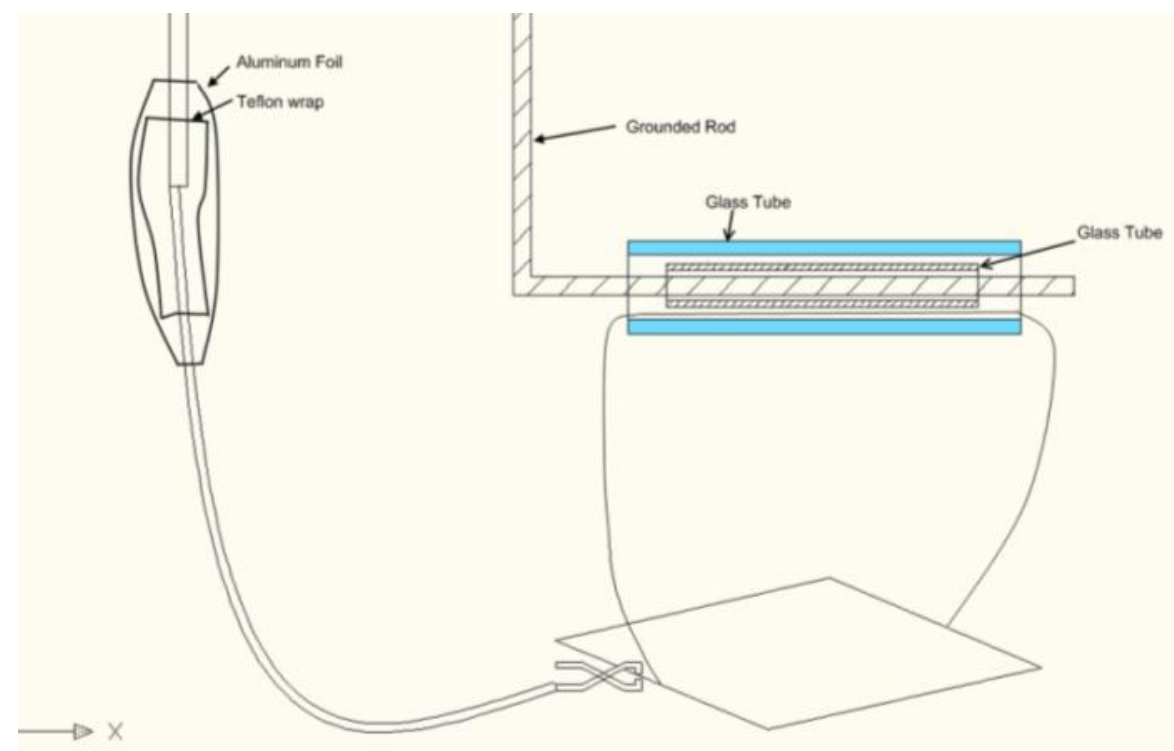

Figure 4.12. A depiction of the electrode setup where two glass tubes insulate the wires holding the panel up.

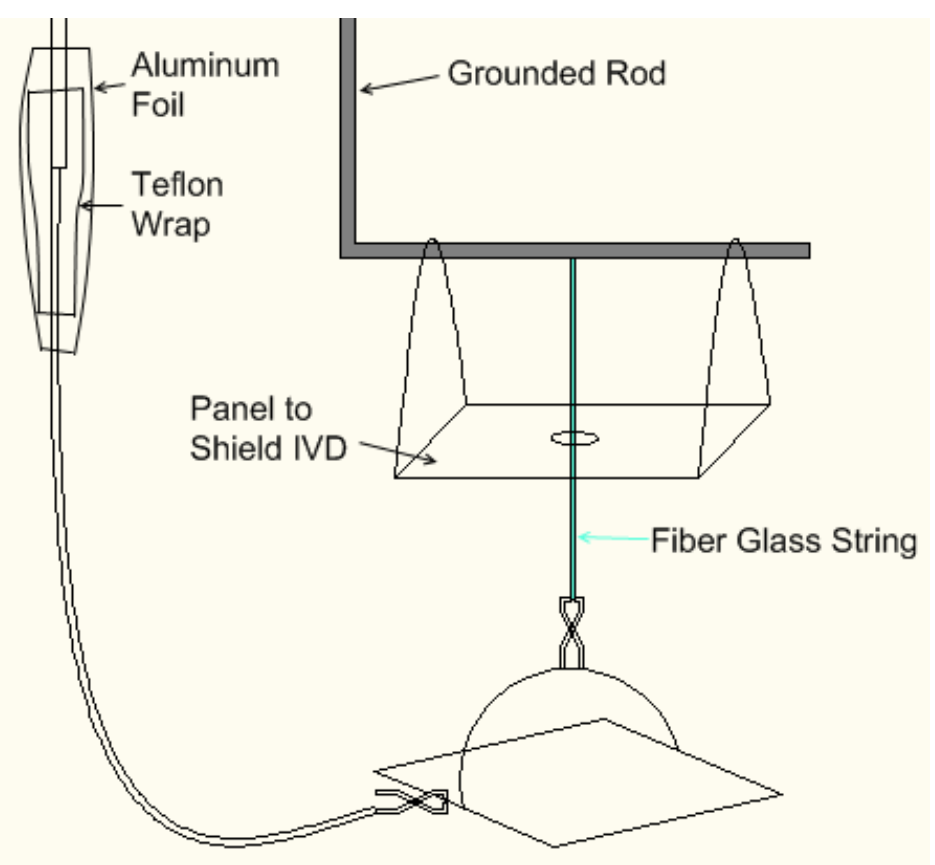

Figure 4.13. A depiction of the electrode setup where the panel is held up by a fiber glass string and IVD is not allowed to coat the string because of a shielding panel. 


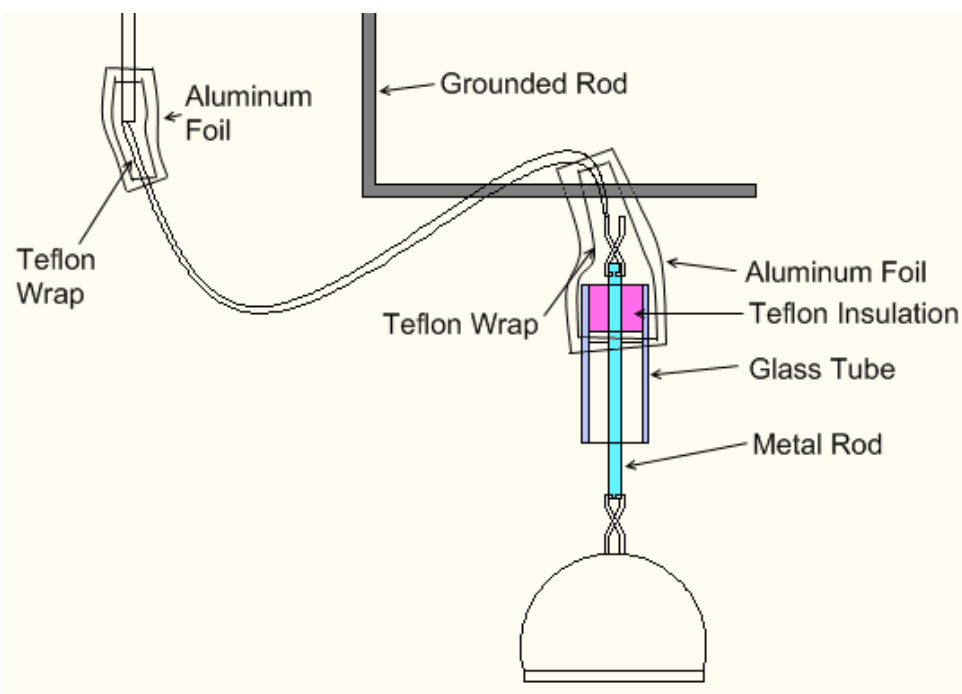

Figure 4.14. A depiction of the electrode setup; the panel hangs from the cathode; the cathode is surrounded by a glass tube so IVD cannot coat the Teflon insulation. 


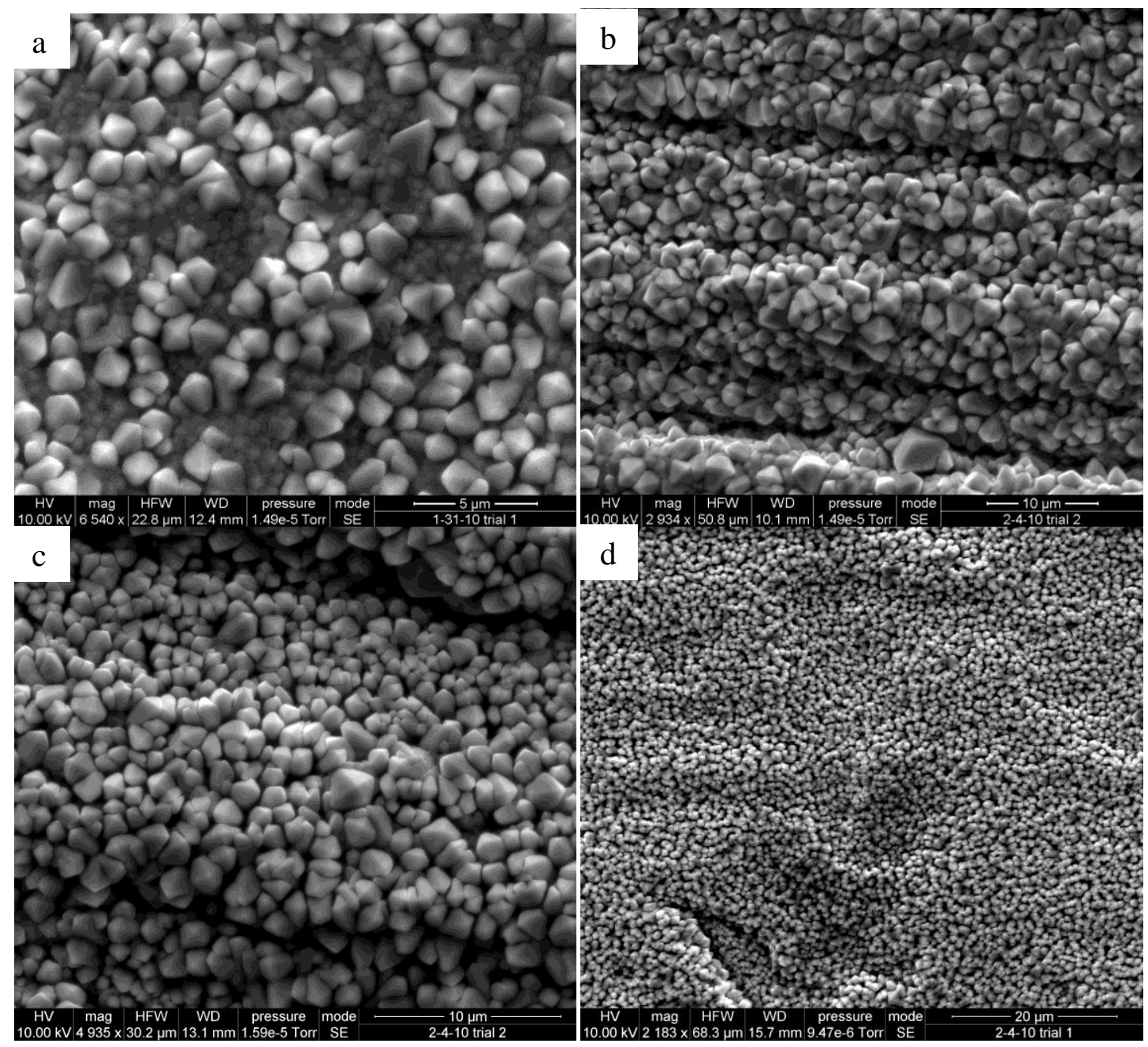

Figure 4.15. SEM pictures of steel panels coated with aluminum from trials where plasma stayed on during evaporation of the aluminum. 


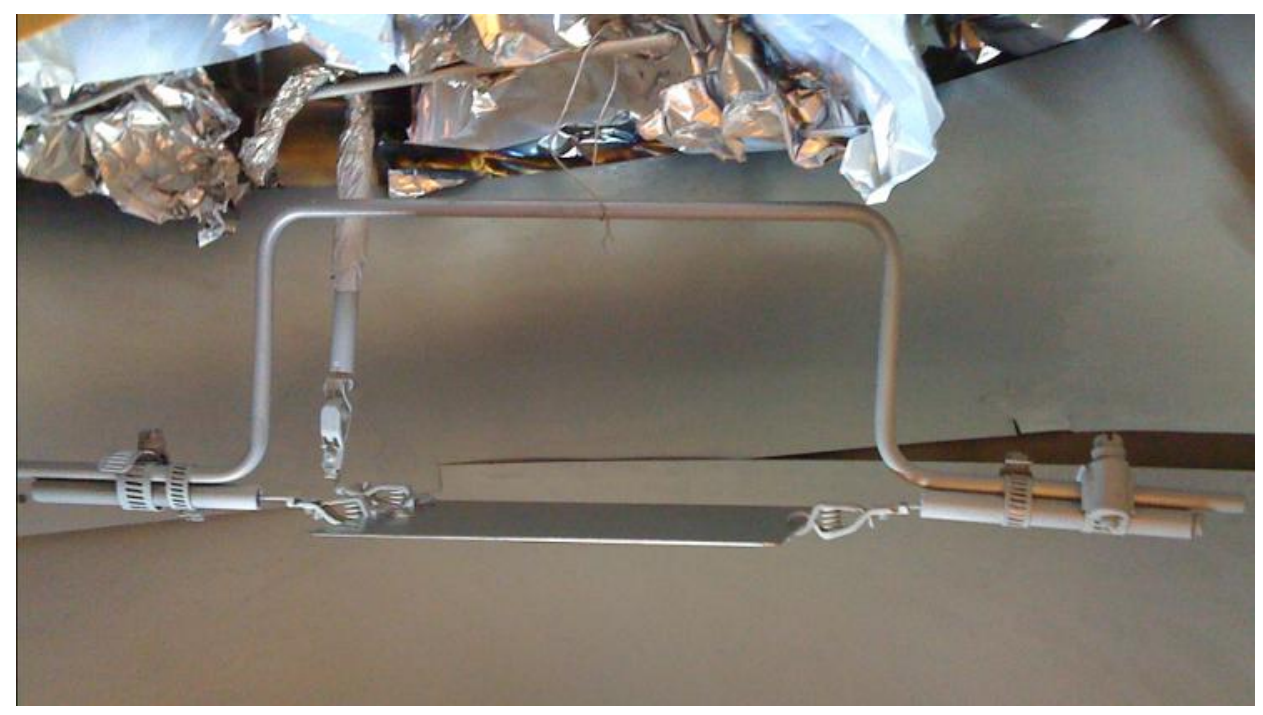

Figure 4.16. Photo of the substrate holder and electrode setup. Teflon spacers located deep within glass tubes keep IVD from coating insulators.

\subsubsection{Attempts at Achieving 100\% IVD Through Modification of Al Feed Rates}

With the correct plasma parameters achieved, an Al coating with mixed structure of IVD and PVD was obtained. Efforts were made in lowering the percentage of PVD and achieving $100 \%$ IVD Al coatings. The wire feed rate was found to be the determining factor on the proportion of IVD to PVD. The summary of results from varied feed rates can be seen in Table 4.1.

Table 4.1. Results of several IVD trials (Plasma: $30 \mathrm{sccm}$ Ar, 2.54kV, $6.5 \mathrm{~mA}$ to $0.05 \mathrm{~mA}$ : IVD: 550A)

\begin{tabular}{llll}
\hline Panel & IVD Conditions & Results & $\begin{array}{l}\text { Thickness } \\
(\boldsymbol{\mu m})\end{array}$ \\
\hline $3 / 22 / 10$ & $\begin{array}{l}4 \mathrm{~min} \sim 5 \mathrm{in} / \mathrm{min} \\
3 / 23 / 10\end{array}$ & $\begin{array}{l}\text { Mix IVD and PVD } \\
\text { to } 18 \mathrm{in} / \mathrm{min} \text { over } \sim 30 \mathrm{sec} .\end{array}$ & $6-8$ \\
& Mix IVD and PVD & $10-18$ \\
$3 / 24 / 10$ & $4 \mathrm{~min} \sim 17 \mathrm{in} / \mathrm{min}$ & PVD & $7-19$ \\
$3 / 25 / 10$ & $2 \mathrm{~min} \sim 8.75 \mathrm{in} / \mathrm{min}$ & Mostly PVD & $3-7$ \\
$3 / 29 / 10$ & $3 \mathrm{~min} 30 \mathrm{sec} \sim 12 \mathrm{in} / \mathrm{min}$ & PVD & $5-14$ \\
$3 / 30 / 10$ & $2 \mathrm{~min} @ 4 \mathrm{in} / \mathrm{min}, 2 \mathrm{~min} @ 8 \mathrm{in} / \mathrm{min}$ & Completely IVD & $1-3$ \\
$3 / 31 / 10$ & $2 \mathrm{~min} @ 4 \mathrm{in} / \mathrm{min}, 2 \mathrm{~min} @ 12 \mathrm{in} / \mathrm{min}$ & Mix IVD and PVD & $4-7$ \\
$3 / 31 / 10$ & $8 \mathrm{~min} @ 4 \mathrm{in} / \mathrm{min}$ & Mix IVD and PVD & $3.2-4.5$
\end{tabular}


Trial 2

4/1/10 2 min@ 4 in/min, 4 min @ $12 \quad$ Mix IVD and PVD 8-13 Trial 1 in $/ \mathrm{min}$

With increasing Al wire feeding rate, higher percentages of PVD structure (individual $\mathrm{Al}$ particles) and less IVD structure (interconnected particles) were found in the resulted $\mathrm{Al}$ coatings, which were shown in figures 4.17-4.20. With this knowledge, a low Al feed rate (4 in/min) was used for the IVD coating process. To achieve coatings of desired thicknesses, a trial was performed for 8 minutes by using $4 \mathrm{in} / \mathrm{min}$ wire feed rate. This also produced PVD like particles, as shown in Figure 4.21. Starting at a low feed rate and ramping to a high feed rate can result in a higher proportion of IVD while keeping a higher deposition rate, as seen in Figure 4.22.

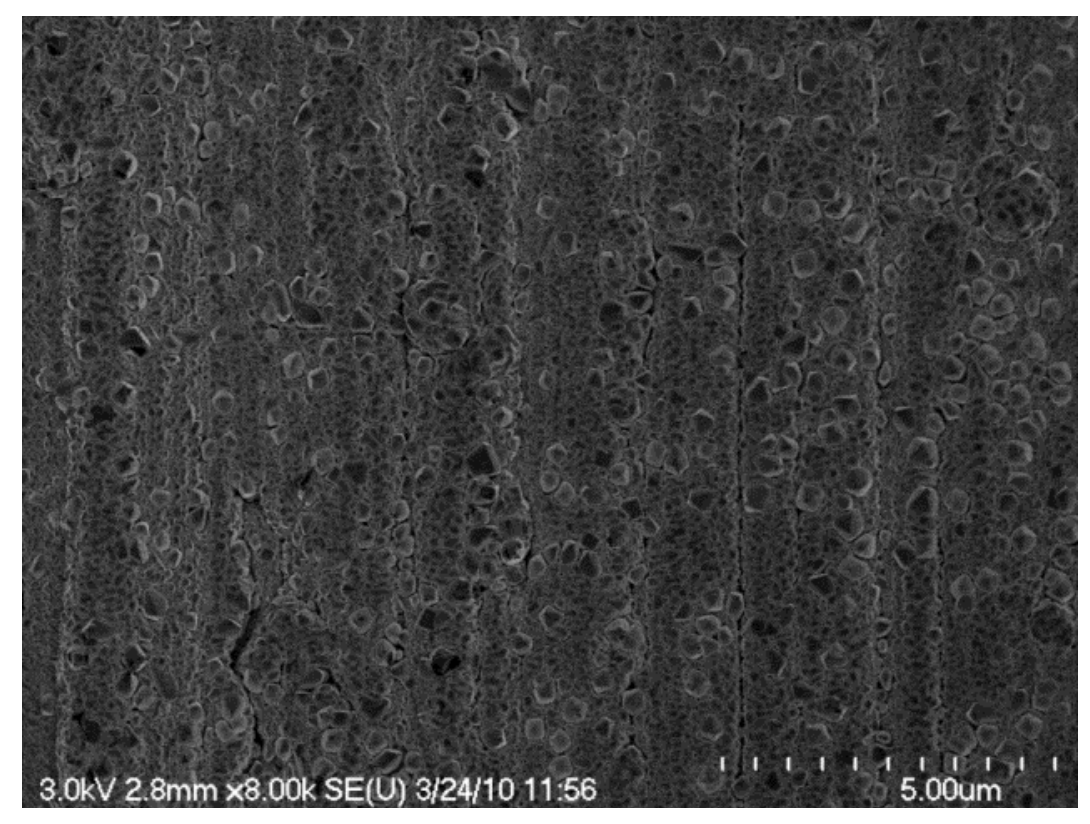

Figure 4.17. SEM image of a panel coated for $4 \mathrm{~min} \sim 5 \mathrm{in} / \mathrm{min}$. 


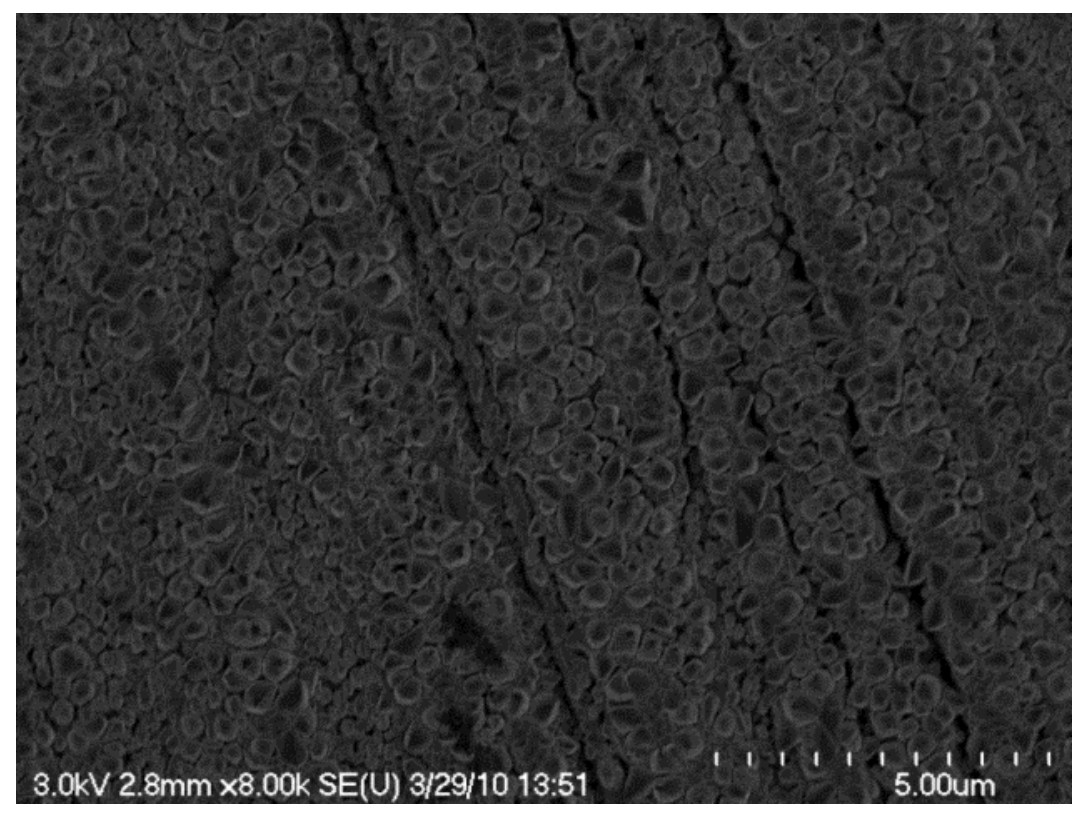

Figure 4.18. SEM image of a panel coated for $2 \mathrm{~min} \sim 8.75 \mathrm{in} / \mathrm{min}$.

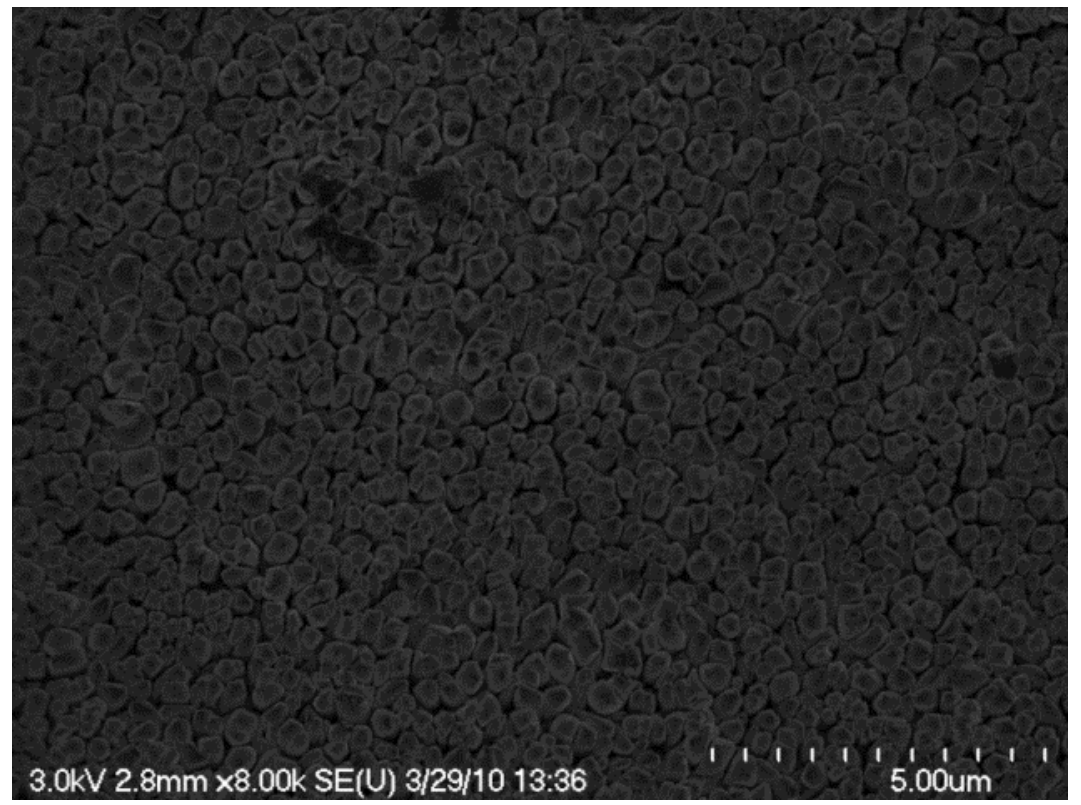

Figure 4.19. SEM image of a panel coated for $3 \mathrm{~min} 30 \mathrm{sec} \sim 12 \mathrm{in} / \mathrm{min}$. 


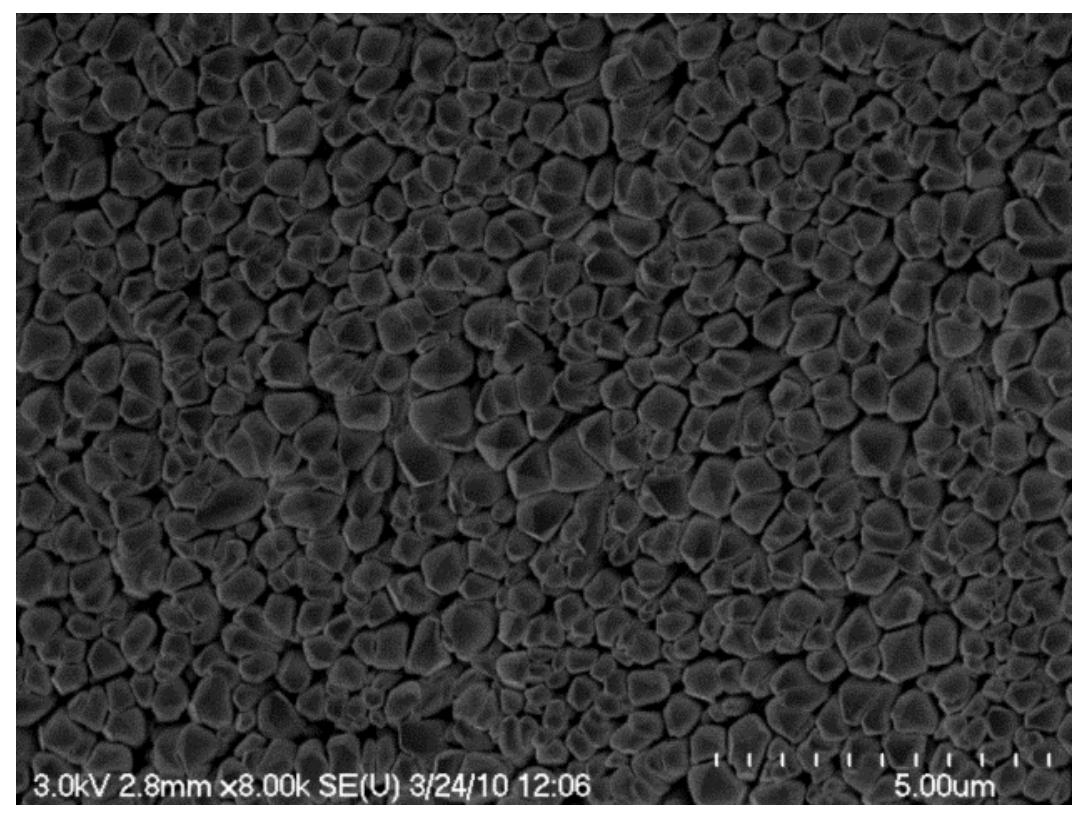

Figure 4.20. SEM image of a panel coated for $4 \mathrm{~min} \sim 17 \mathrm{in} / \mathrm{min}$.

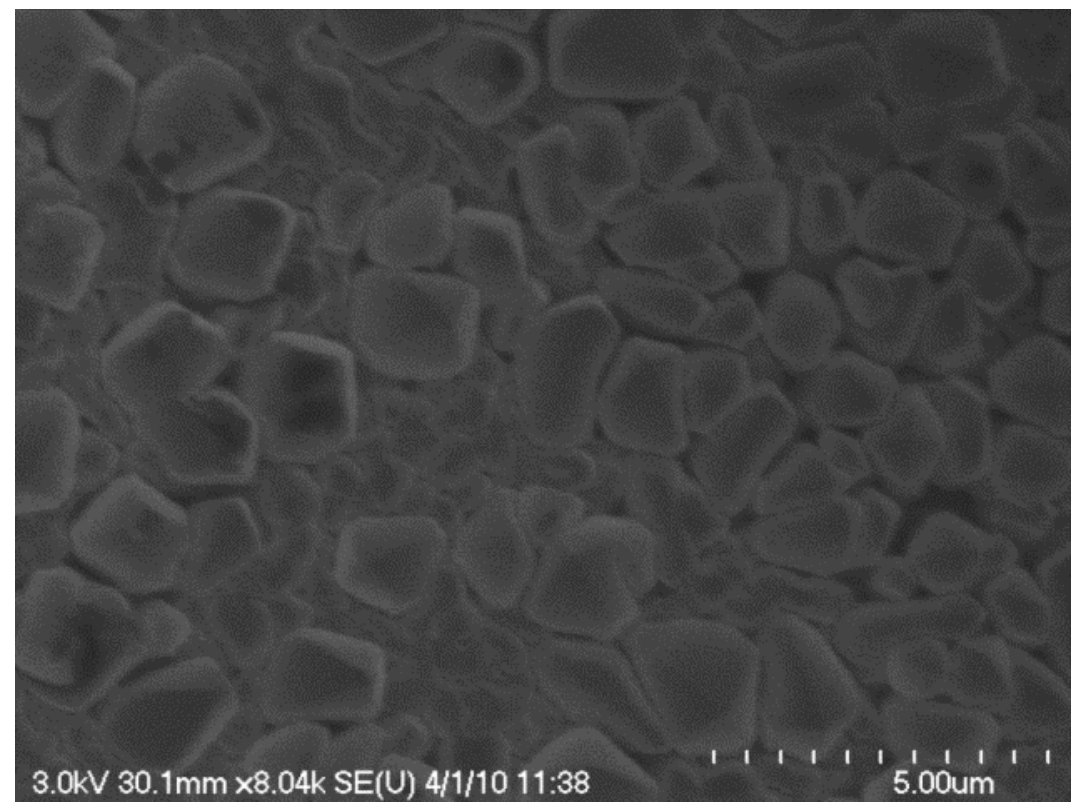

Figure 4.21. SEM image of a panel coated for $8 \mathrm{~min} @ 4 \mathrm{in} / \mathrm{min}(3.2-4.5 \mu \mathrm{m})$. 


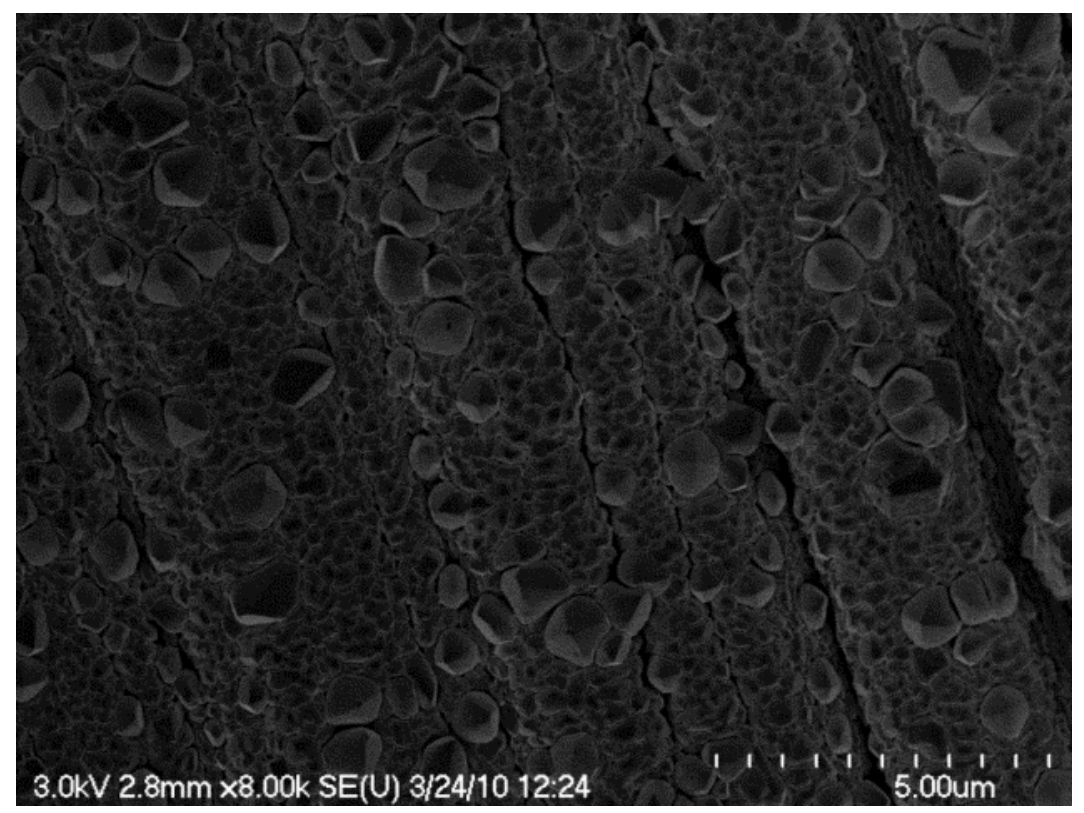

Figure 4.22. SEM image of a panel coated for $3 \mathrm{~min} 10 \mathrm{sec}$ Ramped from $\sim 4 \mathrm{in} / \mathrm{min}$ to 18 in/min over $\sim 30 \mathrm{sec}(3 / 23 / 10)$.

\subsubsection{Determination of Cooling Parameters for Achieving 100\% IVD}

PVD like particles was presumably due to the panel getting too hot, causing coalescence of the particles. Coating the panels in cycles with cooling between cycles resulted in more IVD as shown in Table 4.2. Coating at $4 \mathrm{in} / \mathrm{min}$ for 2 minutes and then at 8 in/min for 2 minutes results in a coating comprised 100\% of IVD, as shown in Figure 4.23. To test if the panel was overheating, panels were coated using the single cycle $100 \%$ IVD conditions, one with a 30 minute delay between cycles, as seen in Figure 4.24, and one with no delay as seen in Figure 4.25. The trial with no delay consisted more of PVD. This supports the conclusion that the panel is overheating. Panels were coated at $4 \mathrm{in} / \mathrm{min}$ for 2 minutes and then $12 \mathrm{in} / \mathrm{min}$ for 2-4 minutes to maximize the deposition rate. The trend of the increasing ratio of PVD to IVD remained so 8 in/min was kept. To decrease the overall trial time, the time at $8 \mathrm{in} / \mathrm{min}$ was increased to 4 and 6 minutes. The increase in time at $8 \mathrm{in} / \mathrm{min}$ resulted in a mixture of IVD and PVD, so 2 minutes at 8 in/min was kept. Another trial was 
performed aimed at reducing coating time by reducing time between cycles. It uses the $100 \%$ IVD single cycle conditions except with a 10 minute break between cycles instead of 30 minutes. It was found that ten minutes between cycles is not long enough for production of $100 \%$ IVD, as shown in Figure 4.26.

Table 4.2. Results of several IVD trials with cooling cycles between coating cycles (Plasma: $30 \mathrm{sccm}$ Ar, $2.54 \mathrm{kV}, 6.5 \mathrm{~mA}$ to $0.05 \mathrm{~mA}$ : IVD: $550 \mathrm{~A}$ )

\begin{tabular}{|c|c|c|c|}
\hline Panel & IVD Conditions & Results & $\begin{array}{l}\text { Thicknes } \\
\text { s }(\mu \mathrm{m})\end{array}$ \\
\hline $\begin{array}{l}4 / 1 / 10 \\
\text { Trial } 2\end{array}$ & $\begin{array}{l}2 \text { min @ } 4 \text { in/min, } 2 \text { min @ } 8 \text { in/min } \\
\text { cycled twice with } 30 \text { minutes between } \\
\text { cycles }\end{array}$ & Completely IVD & $3.3-5.5$ \\
\hline $\begin{array}{l}4 / 6 / 10 \\
\text { Trial } 1\end{array}$ & $\begin{array}{l}2 \text { min @ } 4 \text { in } / \min , 2 \text { min @ } 8 \text { in/min } \\
\text { cycled twice with no time between cycles }\end{array}$ & Mix IVD and PVD & $3.7-5.4$ \\
\hline $\begin{array}{l}4 / 5 / 10 \\
\text { Trial } 2\end{array}$ & $2 \min @ 4$ in $/ \min , 4 \min @ 8$ in $/ \min$ & Mix IVD and PVD & $2.5-4.8$ \\
\hline $\begin{array}{l}4 / 7 / 10 \\
\text { Trial } 1\end{array}$ & $2 \min @ 4$ in $/ \min , 6 \min @ 8$ in $/ \min$ & $\begin{array}{l}\text { Mix IVD and PVD, } \\
\text { worse than } 4 / 5 / 10 \text { trial } 2\end{array}$ & $4.4-5.0$ \\
\hline $\begin{array}{l}4 / 7 / 10 \\
\text { Trial } 2\end{array}$ & $\begin{array}{l}2 \text { min @ } 4 \text { in } / \min , 2 \text { min @ } 8 \text { in } / \text { min with } \\
10 \text { minutes between cycles }\end{array}$ & Mix IVD and PVD & $3.9-6.0$ \\
\hline $4 / 12 / 10$ & $\begin{array}{l}5 \text { cycles of } 2 \text { min @ } 4 \text { in } / \min , 2 \text { min @ } 8 \\
\text { in/min with } 30 \text { minutes between cycles }\end{array}$ & Completely IVD & $8.8-11.9$ \\
\hline
\end{tabular}

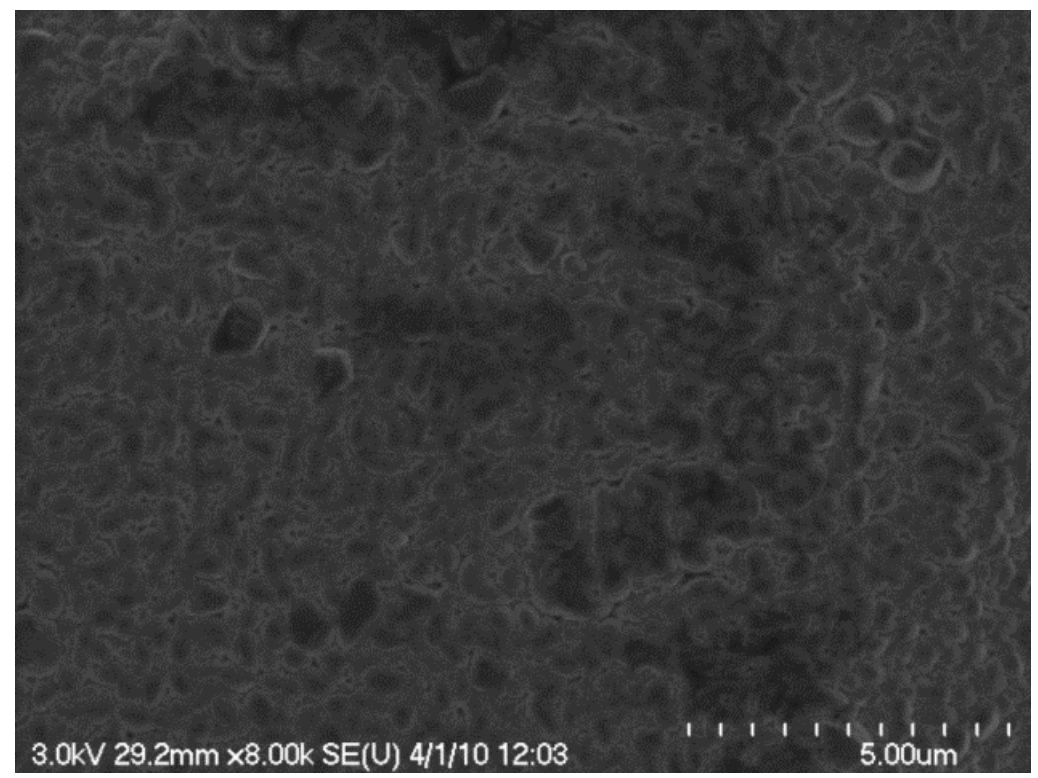

Figure 4.23. SEM image of a panel coated at 2 min @ 4 in/min, 2 min @ 8 in $/ \mathrm{min}(1-3 \mu \mathrm{m})$. 


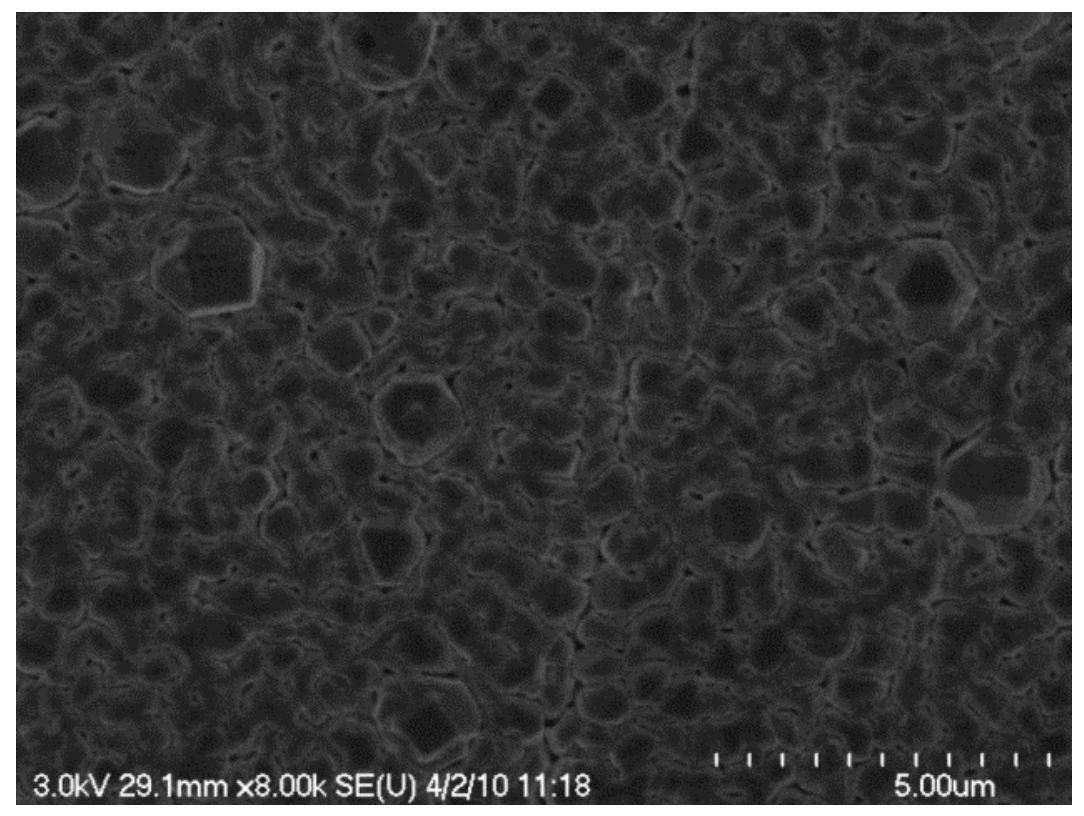

Figure 4.24. SEM image of a panel coated for 2 min @ 4 in/min, 2 min @ 8 in/min cycled twice with 30 minutes between cycles $(3.3-5.5 \mu \mathrm{m})$.

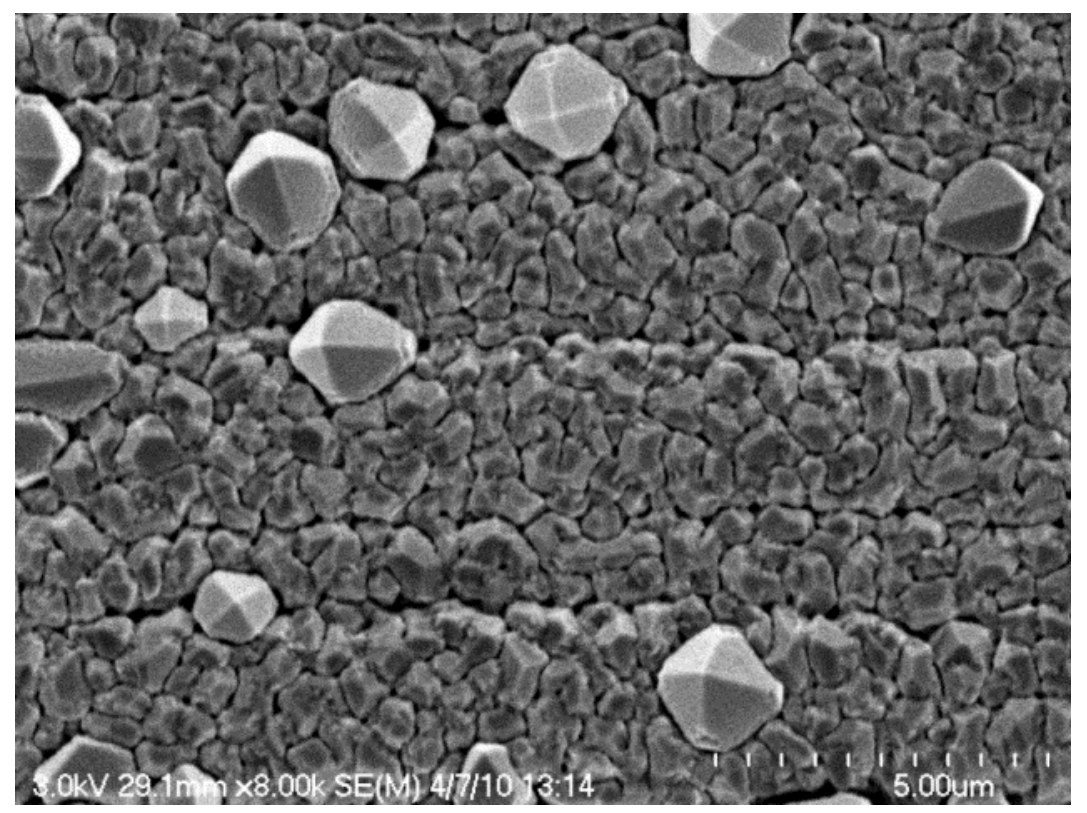

Figure 4.25. SEM image of a panel coated for 2 min @ 4 in $/ \mathrm{min}, 2$ min @ 8 in/min cycled twice with no time between cycles $(3.7-5.4 \mu \mathrm{m})$. 


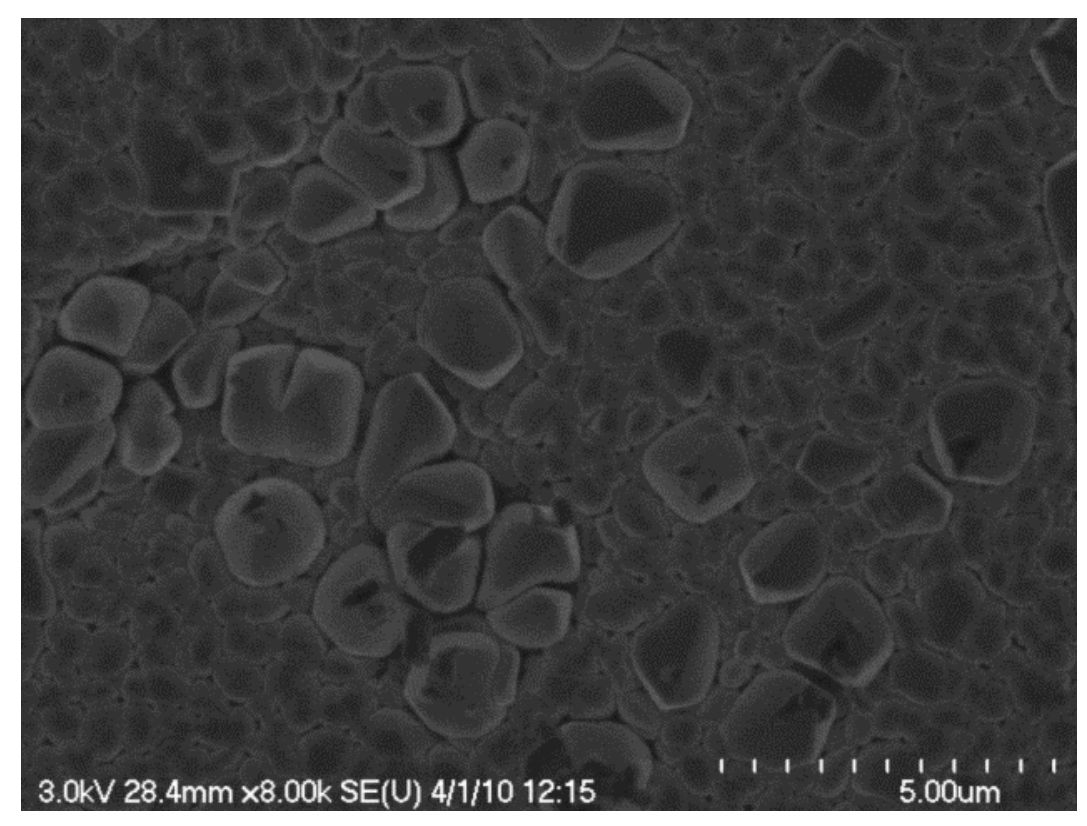

Figure 4.26. SEM image of a panel coated for $2 \mathrm{~min} @ 4$ in $/ \mathrm{min}, 2 \mathrm{~min} @ 8$ in/min cycled five times with 30 minutes between cycles $(8.8-11.9 \mu \mathrm{m})$. 


\subsection{Production and Repeatability of a Class II IVD Al Coating}

The next step was aimed at producing a uniform coating at $13 \mu \mathrm{m}$ thickness using the $100 \%$ IVD parameters found previously. $13 \mu \mathrm{m}$ is a standard thickness at Boeing for corrosion resistant coatings. A panel was coated for five cycles with $100 \%$ IVD parameters and the deposition rate was found to be around $2.5 \mu \mathrm{m} / \mathrm{min}$. The coating remains $100 \%$ IVD after five cycles as seen in Figure 4.27.

After the aforementioned IVD condition was finalized, it was found that the IVD coating thickness had a large variation among trials. One cause that was identified was wire binding up in the wire feeder. The solutions for this was using larger washers on the spool that keeps the wire from getting stuck and the removal of a nozzle resulted in less friction on the wire. There were also problems with the wire feed rate controller that had less accuracy of setting the knob to the correct mark. The range in hash marks was speed 34 for what was thought to be $4 \mathrm{in} / \mathrm{min}$ and speed 37 for $8 \mathrm{in} / \mathrm{min}$ ( $1 \mathrm{in} / \mathrm{min}$ per $3 / 4$ hash marks). Slight variation in the knob setting resulted in large variations in feed rates. To fix this, we adjusted the setting of the wire feeding rate controller. The controller gain was lowered so the change in feed rate per degree of knob turned is lowered. After adjustment, the speed "53" corresponded to $4 \mathrm{in} / \mathrm{min}$ and speed " 65 " corresponded to $8 \mathrm{in} / \mathrm{min}$ ( $1 \mathrm{in} / \mathrm{min}$ per 3 hash marks). Feed rates were more accurately set and it was determined that $5 \mathrm{in} / \mathrm{min}$ was actually being used instead of $4 \mathrm{in} / \mathrm{min}$ as stated in Table 4.1 and Table 4.2 and figures 4.23-4.26. With these changes in effect, two panels were coated to check for uniformity and repeatability. The parameters used were 2 minutes at $5 \mathrm{in} / \mathrm{min}$ and 2 minutes at $8 \mathrm{in} / \mathrm{min}$ each

cycle for 5 cycles. SEM of the panels was performed which verifies $5 \mathrm{in} / \mathrm{min}$ instead of 4 in/min for achieving 100\% IVD microstructure, as shown in Figure 4.27. Panels were 
measured at the locations shown in Figure 4.28 with an Elcometer 355 coating thickness gauge. The panels were coated to satisfy the Class II requirement of at least $13 \mu \mathrm{m}$ thicknesses. Table 4.3 summarizes the coating thickness repeatability. Panel 1 has a coating thickness of $22.9 \mu \mathrm{m}$ with a standard deviation of $3.0 \mu \mathrm{m}$ and Panel 2 has a coating thickness of 19.5 with a standard deviation of $2.5 \mu \mathrm{m}$. It should be noted the accuracy of the measuring device is lowered due to the surface roughness of the panel. When calibrating, the thickness measurement device had a standard deviation of $1.3 \mu \mathrm{m}$. The Elcometer gauge becomes very inaccurate with grit blasted samples due to the high roughness. By coating relatively smooth surfaces, four cycles was determined to be the number of cycles needed for Class II coatings to ensure the $13 \mu \mathrm{m}$ minimum is met.

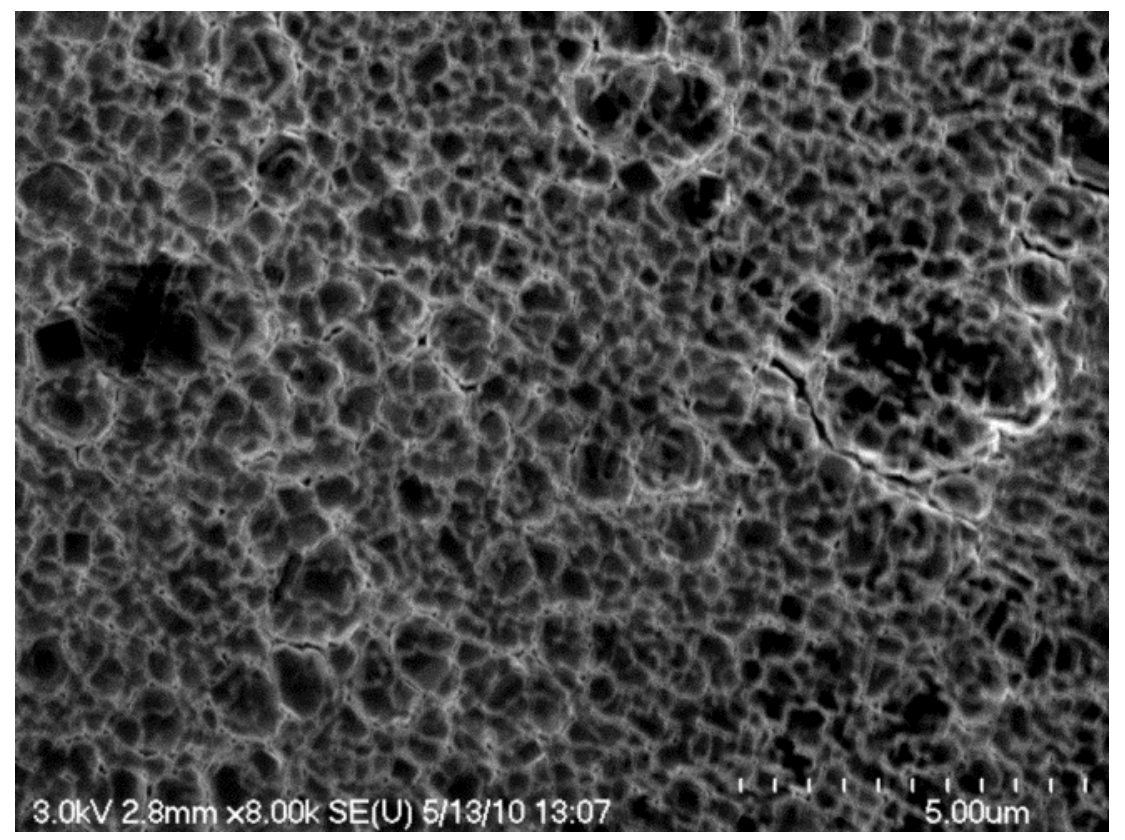

Figure 4.27. SEM image of a panel coated at $5 \mathrm{in} / \mathrm{min}$ for 2 minutes and $8 \mathrm{in} / \mathrm{min}$ for 2 minutes for five cycles. 
Table 4.3. Thickness measurements for test panels at locations shown in Figure 4.28

\begin{tabular}{llll}
\hline Panel 1 Locations & Thickness $(\mu \mathrm{m})$ & Panel 2 Locations & Thickness $(\mu \mathrm{m})$ \\
\hline A & 19.4 & A & 17.9 \\
B & 20.2 & B & 15.3 \\
C & 18.3 & C & 17.4 \\
D & 23.3 & D & 18.2 \\
E & 25.1 & E & 22.2 \\
F & 23.3 & F & 18.6 \\
G & 26.2 & G & 21.9 \\
H & 23.8 & H & 21.4 \\
I & 26.7 & I & 22.2 \\
Average & $\mathbf{2 2 . 9} \pm \mathbf{3 . 0}$ & Average & $\mathbf{1 9 . 5} \pm \mathbf{2 . 5}$ \\
\hline
\end{tabular}

\begin{tabular}{|lll|}
\hline A & B & C \\
D & E & F \\
G & H & I \\
\hline
\end{tabular}

Figure 4.28. Locations of measurements for test panels

IVD thickness was measured using SEM, as seen in Figure 4.29. Optimum conditions to achieve Class II coating thickness was found to be 2 min @ 4 in/min, 2 min @ 8 in/min cycled five times with 30 minutes between cycles for cooling in argon purge. 

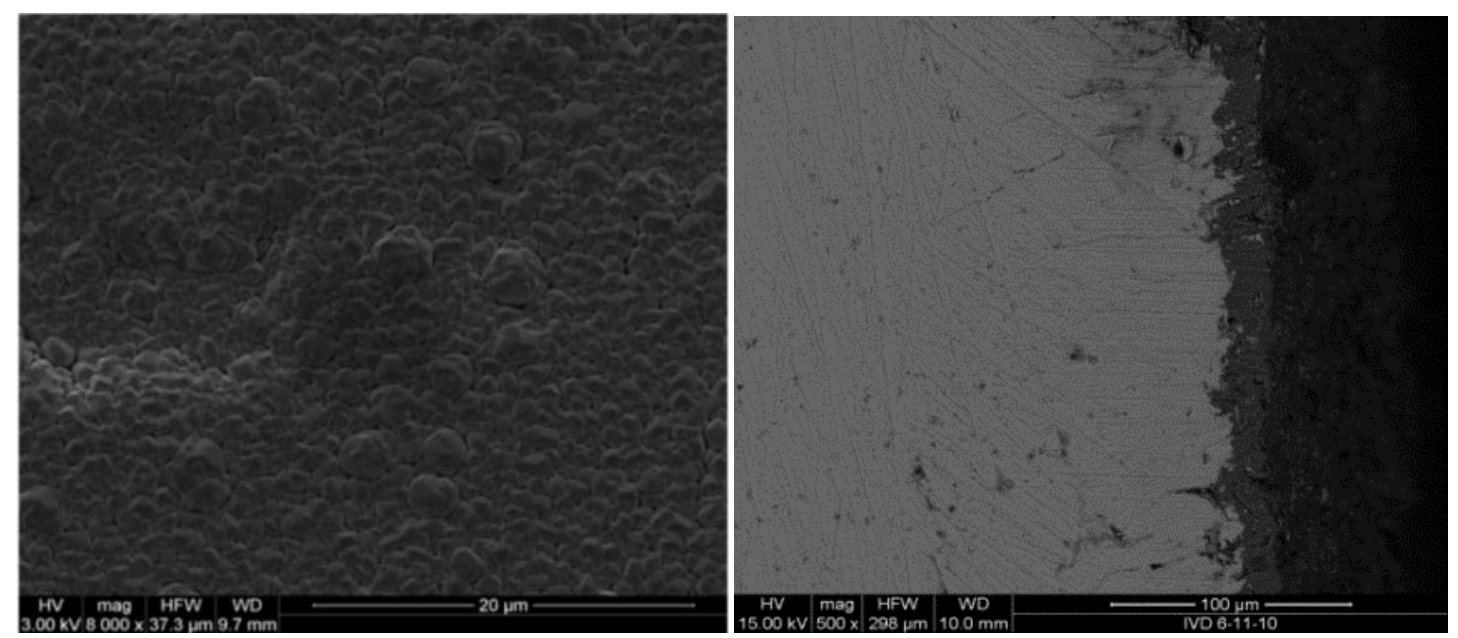

Figure 4.29. SEM image of IVD Al coated steel, from seen from the top (left) and from the cross section (right).

\subsection{Application of Plasma Nanocoating}

Coating thickness of plasma nanocoating cannot be measured on IVD. Instead, panels of the same surface area have silicon wafers attached throughout. Coating thickness was measured on the silicon wafers by a null seeking type AutoEL-II automatic ellipsometer with 632.8 hellium-neon laser light source. Figure 4.30 shows the thicknesses and their refractive indexes of the plasma coatings from TMS/Ar gas mixture with deposition time. Coating thicknesses and refractive indices showed nearly a linear dependence on plasma deposition time. 3 min plasma deposition gave rise to a $\sim 50 \mathrm{~nm}$ coating thickness with refractive index of 1.7. The coating thickness uniformity on a $4 \times 6$ inch steel panel is shown in Figure 4.31 . As seen from the plot, the plasma coating was very uniform in both vertical (6 in direction) and horizontal (4 in direction) directions with slight increases in thickness near the edges. Coatings become thicker near corners and edges due to the local increase of electric field, otherwise known as field enhancement. Increased coating thickness was apparent by the difference in color near the edges of the panel shown in Figure 4.32. 


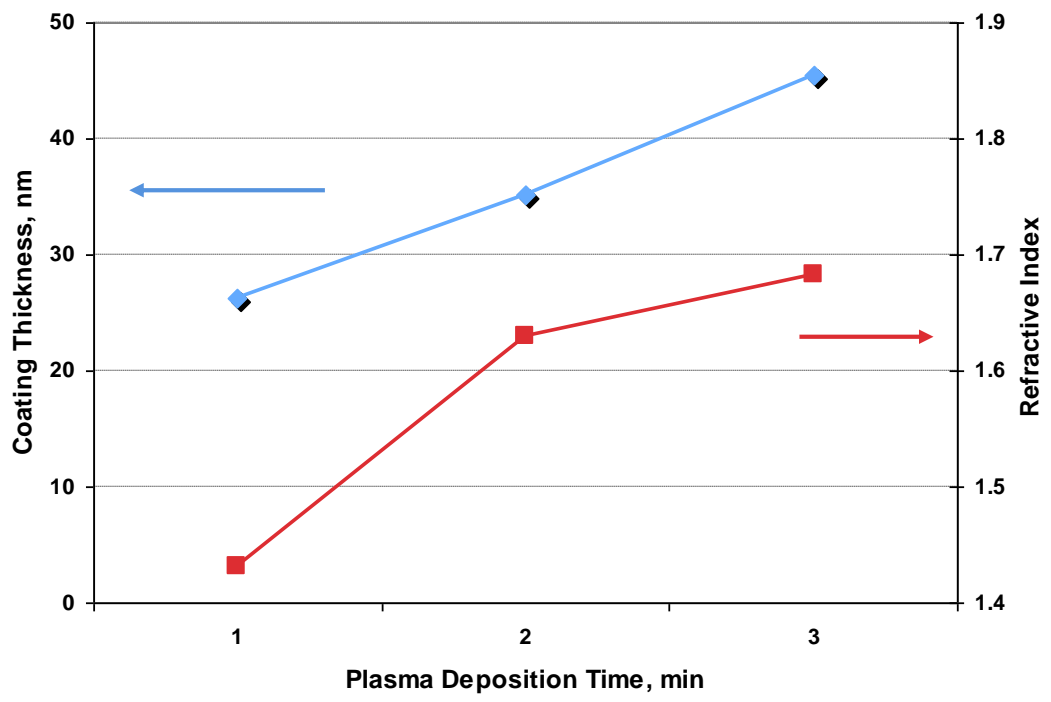

Figure 4.30. Deposition time dependence of plasma coating thickness and refractive indices. Plasma conditions were: $1 \mathrm{sccm}$ argon, $1 \mathrm{sccm}$ TMS, 50 mTorr system pressure, - $900 \mathrm{~V} \mathrm{DC}$ potential.
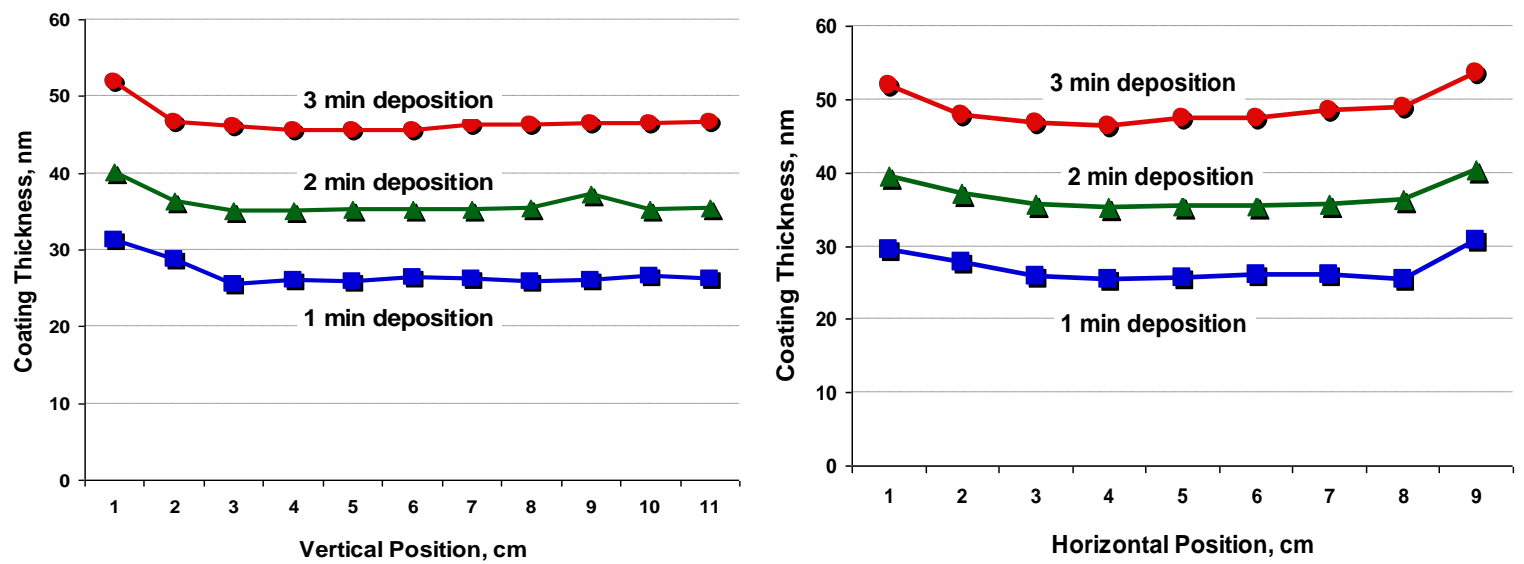

Figure 4.31. Plasma coating uniformity on a $4 \times 6$ inch steel panel in vertical (6 inch direction) and horizontal (4 inch direction) positions. Plasma conditions were: $1 \mathrm{sccm}$ argon, $1 \mathrm{sccm}$ TMS, 50 mTorr system pressure, - 900 V DC potential. 


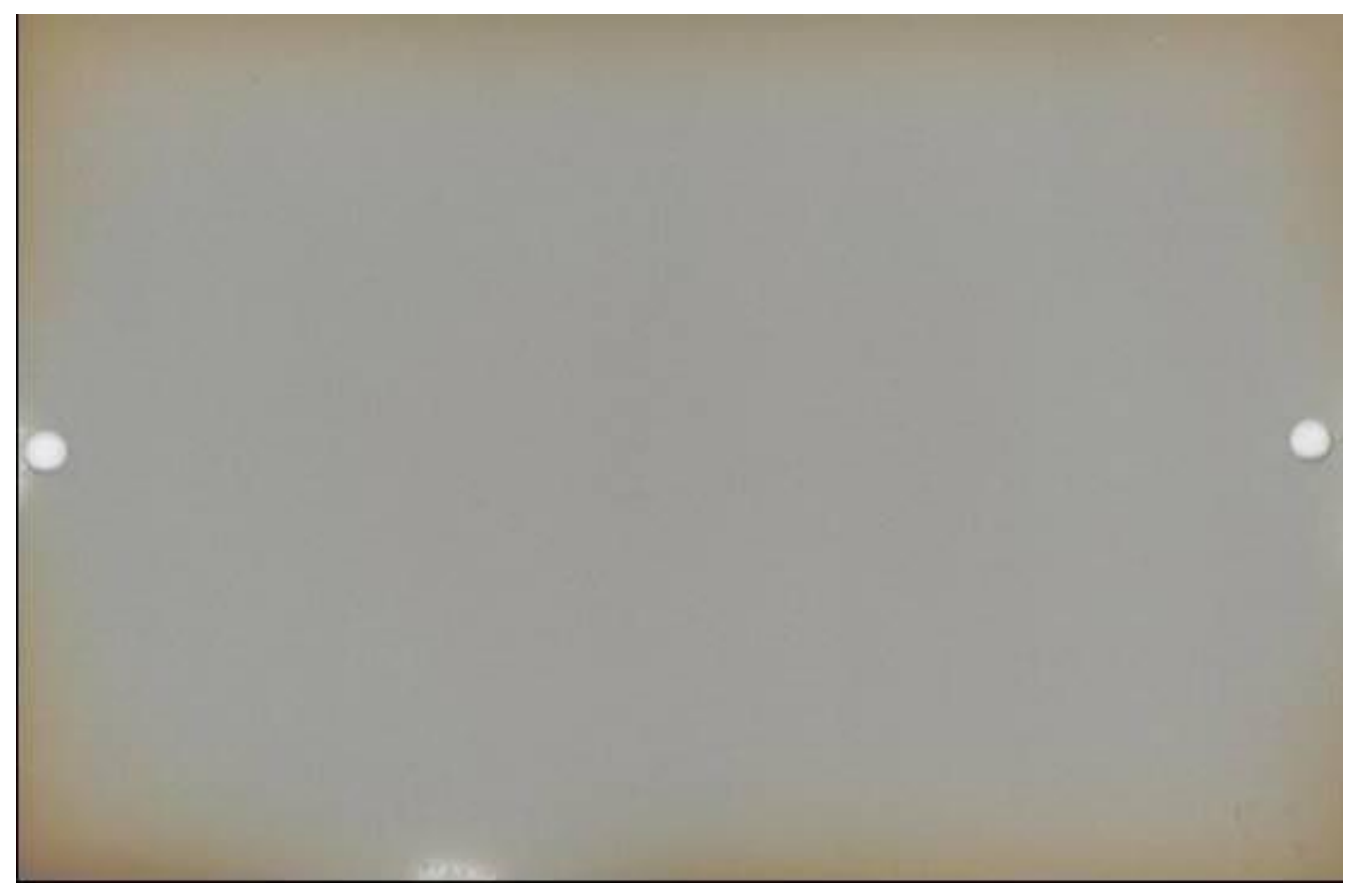

Figure 4.32. IVD coated panel with subsequent plasma coating.

Plasma deposition in a DC glow discharge is normally dependent upon the sample size or surface area of the specimens. In this project, this phenomenon was further examined and confirmed. Figure 4.33 shows the dependence of plasma coatings on specimen surface areas. It can be seen that plasma coating thickness increased with decrease of the sample size or surface area. This phenomenon was taken into account during this project to ensure plasma coatings with consistent thicknesses. 


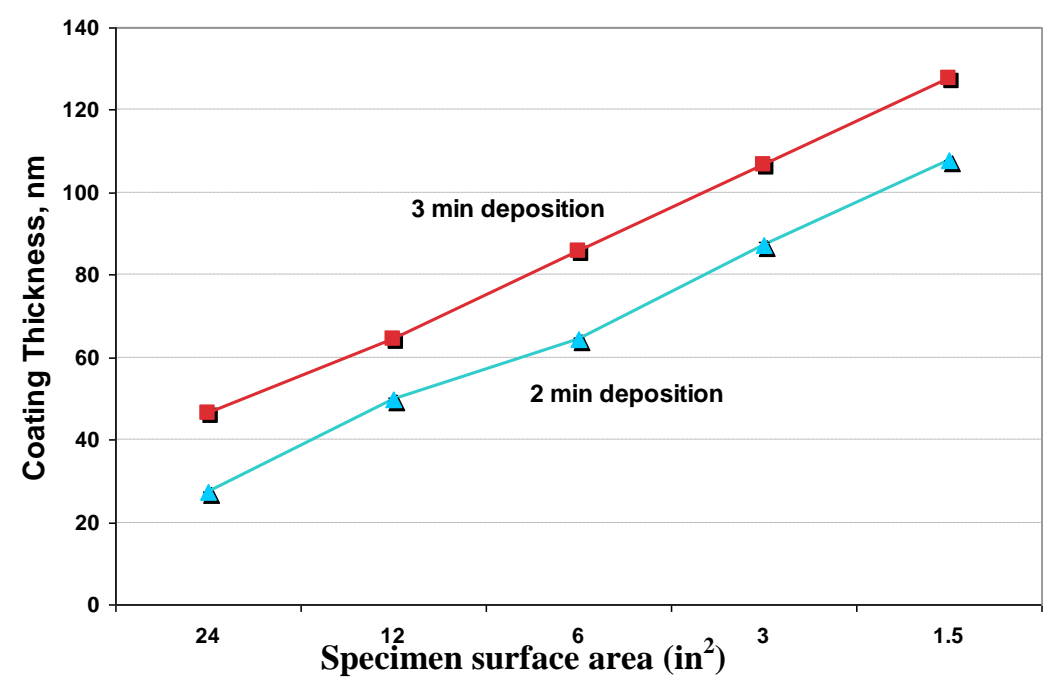

Figure 4.33. Dependence of plasma coatings on specimen size. Plasma conditions were: 1 sccm argon, 1 sccm TMS, 50 mTorr system pressure, - $900 \mathrm{~V}$ DC potential.

\subsection{Evaluation of Possible Contamination of IVD from Plasma Coating}

A major concern for Boeing was the possibility of contamination of their industrial sized IVD reactor by plasma coating over spray, e.g. contamination being incorporation of plasma coating particulate into the IVD Al. To test for this, energy dispersive spectroscopy (EDS) of panels coated prior to and after plasma coating samples was performed and composition analyzed, as shown in Table 4.4. EDS showed no increase in carbon or silicon atomic concentrations after plasma coating processes. Slightly higher carbon contamination of IVD coated in our coater compared to Boeing's coater was found. The slightly higher increase in carbon is not due to the polymer coating process. It was determined to be caused by crucible dry-out at the end of the coating process as the crucible is cooling. The plasma coating process was found to coat the sample holders as shown in figures 4.34-4.36. Electrode holders may be replaced easily in industrial IVD coating. In case there are some parts that Boeing would like to be able to clean and reuse, 2:1 oxygen to argon plasma 
cleaning for 10 minutes at 50 mTorr was attempted. Oxygen + argon plasma cleaning shows significant cleaning, but total removal of plasma coating was not achieved.

Table 4.4. Atomic concentration percentage of IVD Al coating surfaces obtained by EDS.

\begin{tabular}{|c|c|c|c|c|}
\hline Sample & Al & $\mathbf{O}$ & $\mathrm{C}$ & $\mathbf{S i}$ \\
\hline $\begin{array}{l}\text { IVD Al coating } \\
\text { ( deposited before TMS coatings were tried) }\end{array}$ & 93.8 & 4.2 & 2.0 & 0.0 \\
\hline $\begin{array}{l}\text { IVD Al coating } \\
\text { (after TMS plasma deposition was performed) }\end{array}$ & 93.8 & 4.2 & 2.1 & 0.0 \\
\hline $\begin{array}{l}\text { IVD Al coating } \\
\text { (after TMS plasma deposition was performed) }\end{array}$ & 90.3 & 7.0 & 2.7 & 0.0 \\
\hline $\begin{array}{l}\text { IVD Al coating (deposited after oxygen plasma } \\
\text { cleaning) }\end{array}$ & 95.4 & 3.0 & 1.6 & 0.0 \\
\hline IVD Al from Boeing & 91.3 & 7.9 & 0.8 & 0.0 \\
\hline
\end{tabular}

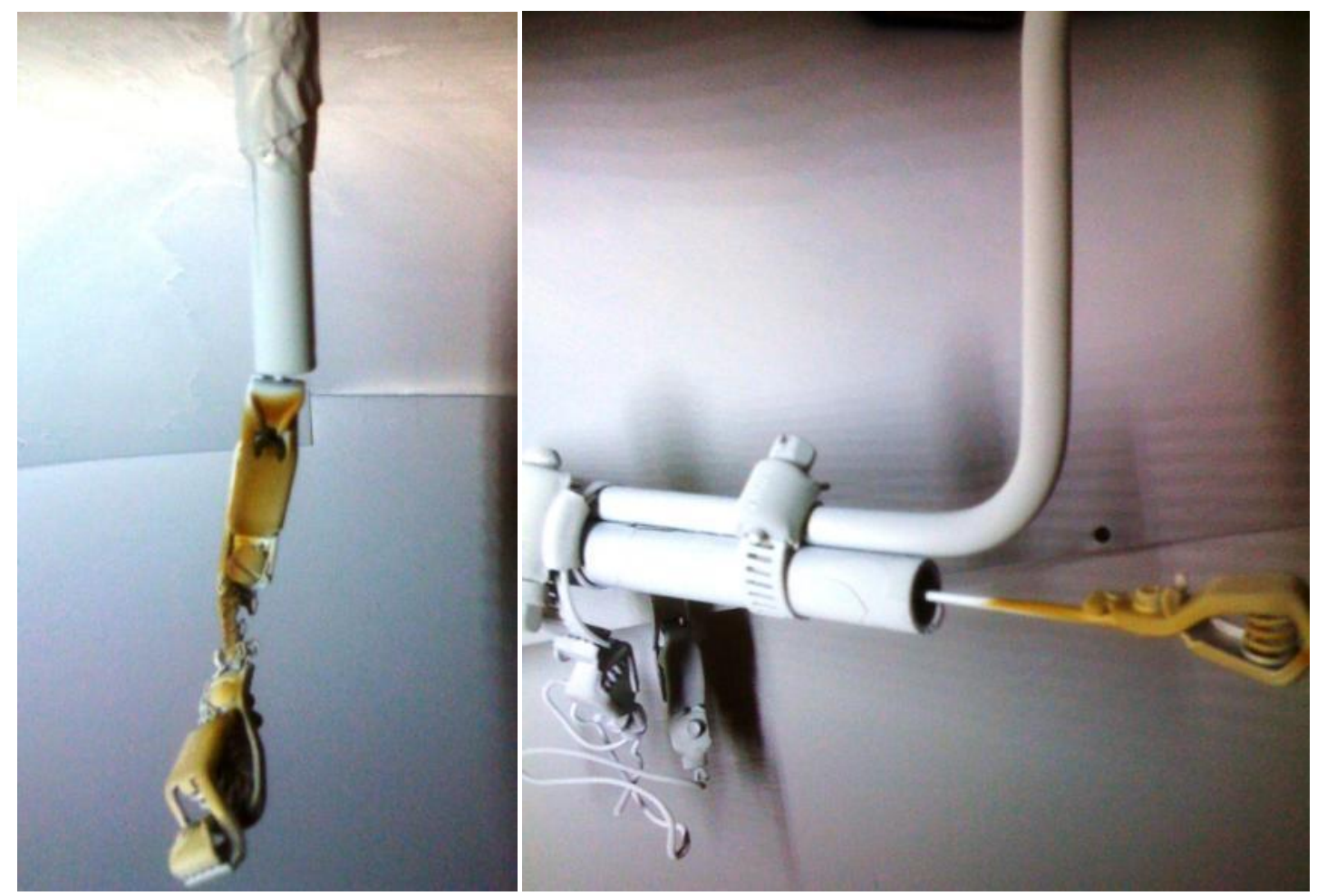

Figure 4.34. Images of panel holder electrodes after plasma coating. 


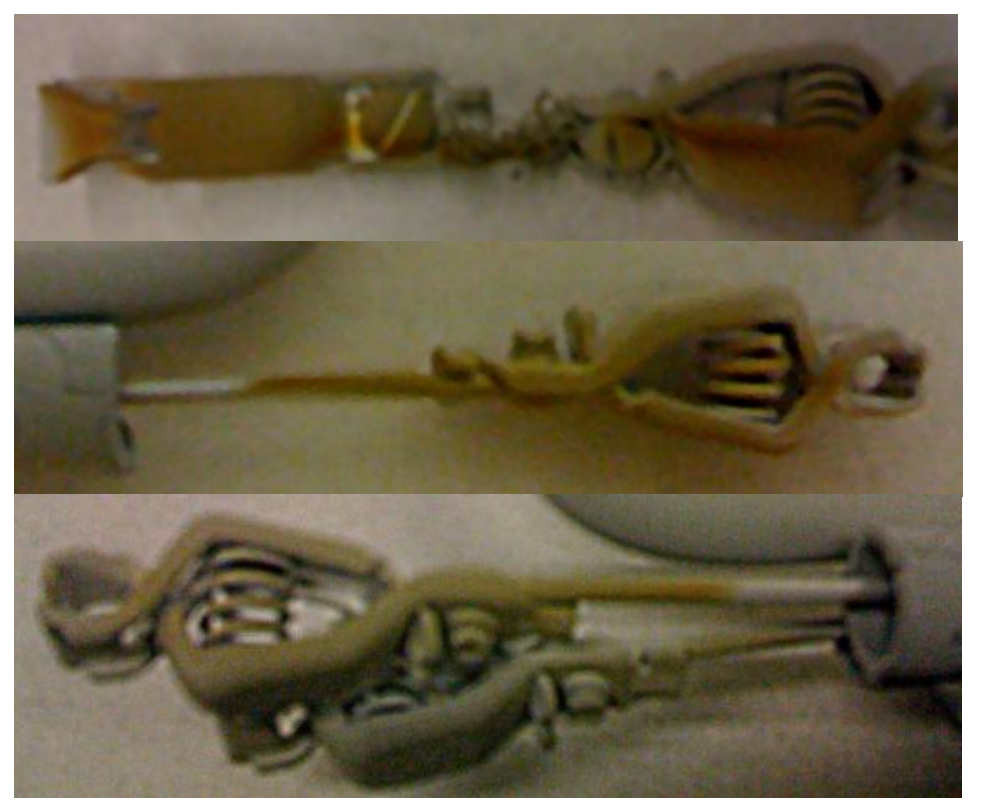

Figure 4.35. Images of electrode/panel holders for IVD Al and TMS plasma coatings after IVD Al and TMS plasma coating.

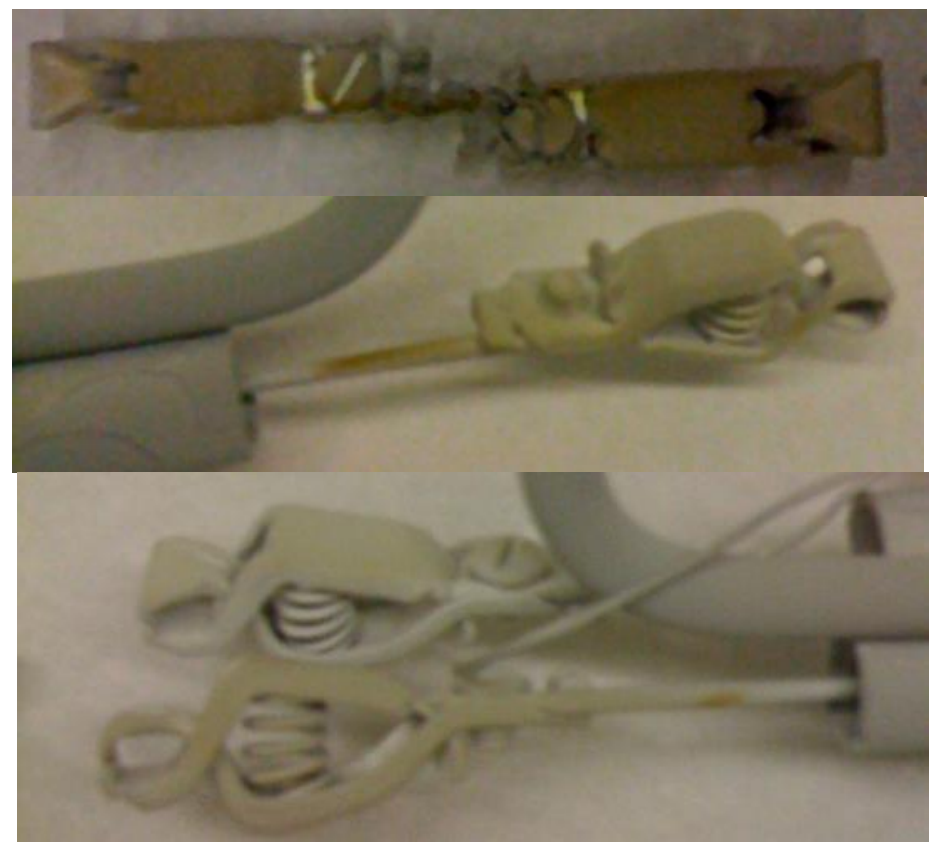

Figure 4.36. Images of electrode/panel holders for IVD Al and TMS plasma coatings after 2:1 oxygen to argon plasma cleaning for 10 minutes at 50 mTorr. 


\subsection{Conclusion}

A hybrid IVD and plasma coater reactor was successfully constructed. Class I thickness IVD Al coatings were achieved. Coatings were repeatable in thickness and structure by using cycles of deposition and cooling. $50 \mathrm{~nm}$ thick plasma coatings were applied to the IVD. This is an estimate from coating on silicon wafers; plasma coating thickness cannot be determined atop of IVD. Coating did not contaminate subsequent IVD coatings, as shown by EDS. Incorporation of plasma coating into the IVD reactor is simple due to high vacuum required by the IVD process, therefore conversion of IVD reactors already in use should require minimal modifications. 


\subsection{References}

1. Conrads, H., Schmidt, M., 2000, "Plasma generation and plasma sources," Plasma Sources Sci. Technol., 9, 441-454.

2. Bogaerts, A., Neyts, E., Renaat, G., Van der Mullen, J., 2002, "Gas discharge plasmas and their applications," Spectrochimica Acta Part B, 57, 609-658.

3. Holmes, V.L., Bridger, M., 2004, “ Aluminum coatings turn green: IVD process delivers environmentally friendly corrosionprotection," Metal Finishing, 102, 6-10.

4. Helmersson, U., et al., 2006, "Ionized physical vapor deposition (IPVD): A review of technology and applications," Thin Solid Films, 513, 1-24.

5. Aisenberg, S., Chabot, R.W., 1973, "Physics of Ion Plating and Ion Beam Deposition," J. Vac. Sci. Technol., 10, 104-107.

6. Mattox, D.M., 1973, “Fundamentals of Ion Plating,” J. Vac. Sci. Technol., 10, 47-52.

7. Thornton, J., 1977, "High Rate Thick Film Growth,” Ann. Rev. Mater. Sci., 7, 239260.

8. Schiller, S., Heisig, U., Goedicke, K., 1975, "Alternating ion plating- A method of high-rate deposition," J. Vac. Sci. Technol., 12, 858-864.

9. Lichter, S., Chen, J., 1986, "Model for Columnar Microstructure of Thin Solid Films," Physical Review Letters, 56, 1396-1399.

10. Yoyisha, M. Wada, K., Jang, B.K., Matsubara, H., 2004, "Computer simulation of nano-pore formation in EB-PVD thermal barrier coatings," Surface and Coatings Technology, 187, 399-407. 


\section{Chapter 5 Corrosion Protective Coatings Produced with a Hybrid}

\section{IVD-Plasma Coater}

Abstract: This study investigated the incorporation of an ion vapor deposition (IVD) chamber with a plasma nanocoating chamber in one single hybrid coater for production of corrosion resistant coatings. A direct current cathodic plasma process was used to produce trimethylsilane (TMS) plasma nanocoatings on steel substrates coated with an ion vapor deposited aluminum layer (IVD Al). TMS coatings were created as an environmentally friendly replacement to toxic chromate conversion coating. Due to the similarity in the IVD and plasma coating processes, the incorporation of both processes into one chamber was evaluated as a cost savings measure. A hybrid coating chamber was constructed to examine the possibility of contamination between the two processes and to elucidate the effect of oxidation of the IVD Al as it pertained to corrosion resistance. Based on corrosion current measured by anodic potentiodynamic polarization, the order of corrosion resistance from high to low was: TMS plasma coated IVD with a naturally formed oxide layer, TMS plasma coated IVD without an oxide layer, bare IVD. $\mathrm{SO}_{2}$ salt spray corrosion resistance tests were performed per ASTM G85-94, annex A4. IVD Al with TMS plasma coatings performed similarly to chromate conversion coated samples with a passing grade as determined by corrosion byproducts. Plasma coating and IVD coating processes did not affect each other, i.e., their individual performance was retained as observed after salt fog corrosion testing. Further, there was no contamination to the reactor or on the subsequent coatings as observed by energy dispersive spectroscopy (EDS). All IVD coated panels coated subsequent to 
plasma coating trials passed primer adhesion testing MIL-PRF-85582 Class C2 per ASTM D 3359.

\subsection{Introduction}

A standard practice in the aerospace industry is to apply thin coatings of aluminum to high strength steel parts to act as a corrosion resistant barrier. Often, these aluminum coatings are applied using the ion vapor deposition (IVD) technique, whereby aluminum is evaporated under high vacuum and allowed to condense on the steel part [1]. The part is surrounded by a plasma in order to produce a more dense microstructure. The resulting microstructure is further densified by glass bead peening, but remains too porous even after applications of paint. For decades, the industry standard has been to apply a chromate conversion coating by electroplating in order to seal the IVD and as a method to provide good adhesion to subsequent primer paints. Chromate conversion coatings are known to be carcinogenic and its use has been banned, therefore an environmentally benign replacement for post treatment of IVD Al is needed [2].

Organosilane plasma coatings have emerged as an environmentally friendly and effective alternative to the toxic chromate coatings for corrosion resistance of aircraft aluminum alloys [3-7]. Previous papers have shown plasma coatings, produced through plasmas containing organosilicate monomers, increase corrosion resistance when applied as a thin coating on various metal substrates [3,5,8-11]. Further, strong adhesion between plasma polymers and organic paint primers as well as aluminum substrates has been observed $[5,7,12,13]$. In the cathodic plasma coating process, a substrate is maintained at a high negative potential (cathode). A monomer is dissociated into fragments by impacting ions, electrons, and excited species to form free radicals. These free radicals react with the 
substrate and each other to deposit a plasma coating that forms a tight network of covalent bonds with low permeability. The resulting plasma coatings can thus act as a barrier to keep corroding agents from reaching the metal substrate.

The components of an IVD reactor are very similar to that of the organosilane plasma coating reactor, so to save time, space, and reduce cost, it was hypothesized that the two processes could be combined into one hybrid coater. Both coatings are:

- applied in vacuum;

- the substrate is the cathode;

- the plasma is discharged around $1 \mathrm{kV}$.

The IVD reactor requires a base pressure of 0.08 mTorr while the plasma coating only requires 1 mTorr, so vacuum pumps used for IVD will also work for plasma coating. The only added component to the reactor system would be the organosilane gas cylinder and relevant mass flow controllers. This research is a lab scale demonstration of the hybrid coating chamber to study the feasibility of incorporating the plasma coating process into industry scale IVD coaters currently used in production lines.

Our previous study showed that organosilane plasma coatings had improved corrosion protection of IVD coated steel aluminum alloys. In those experiments, The Boeing Company provided IVD Al coated steel panels that were shipped to our lab and subsequently plasma coated. Once the IVD Al coating was exposed to atmosphere, a natural oxide layer was quickly formed on its surface. We were therefore interested in whether or not the oxide layer was necessary to the corrosion resistance. If not, the process should be eliminated to save on production cycle time. Also, we were interested in whether the two processes affect each other in terms of cross-contamination when combined in one chamber. To test this, the 
hybrid coating chamber needed to incorporate both coating systems such that the two processes could be performed in a continuous process without the loss of vacuum.

\subsection{Experimental}

\subsubsection{Materials}

The Boeing Company provided 4340 steel panels with dimensions $2 " \times 6$ " $\times 0.04$ " for electrochemical testing and $4 " \times 6 " \times 0.04$ " for corrosion resistance testing. The panels were cleaned by The Boeing Company with an alkaline cleaner, pickling solution, and grit blasted. Panels were shipped overnight and had additional cleaning with acetone wiped with Kimwipes in our lab before use. TMS monomer gas was procured from Gelest Inc. with 97\% minimum purity. Oxygen $\left(\mathrm{O}_{2}, 99.9 \%\right)$ was procured from Airgas. Argon (Ar, 99.9\%) was procured from Praxair. Gases were used as received without further purification. 1/16" aluminum 1100 wire, produced by HQA Wire Products, was used for IVD Al coating.

\subsubsection{IVD Coater/Plasma Reactor}

A cylindrical metal stainless steel reactor chamber with dimensions of $2^{\prime} \times 2^{\prime}$ (diameter $\times$ height) was built for this study as depicted in Figure 1. The chamber is pumped down to a base pressure of 1 mTorr using an Edward EH series vacuum pump and model EH500A booster pump. A Varian VHS series diffusion pump was used to achieve a further vacuum of $0.02 \mathrm{mTorr}$. Argon is flowed into the reactor at $1 \mathrm{sccm}$ (standard cubic centimeter per minute) to reach a preset pressure of 9 mTorr. A MKS mass flow meter (Model 247 C) was used for monitoring the gas flow rate and a MKS pressure controller (Model 252) was used to control the gas pressure in the reactor chamber. After the system pressure stabilized to a preset value, direct current (DC) power was supplied to the substrate by a Spellman 
SL60 DC power supply controlled in voltage mode. A WPI Power Systems Inc. 8 kVA SCR controlled power supply was used to heat a Momentive Performance Materials heating boat. Aluminum wire was fed into the heating boat and vaporized. After IVD coating, the chamber was allowed to cool while purged with argon at 9 mTorr for 45 minutes. Samples were plasma coated as described in Table 5.1. The IVD Al surface morphology was analyzed with the FEI Quanta 600 FEG Extended Vacuum Scanning Electron Microscope (SEM). SEM confirms IVD structure of deposited aluminum, as shown in Figure 5.2. IVD coating was measured at the cross section to be around $13 \mu \mathrm{m}$ in thickness.

Table 5.1. Procedure to coat IVD Al coated steel with TMS plasma coating with and without oxide layer.

\begin{tabular}{ll}
\hline Sample Identifier & Plasma Coating Procedure \\
\hline $\begin{array}{l}\text { IVD/ } / \mathrm{Al}_{2} \mathrm{O}_{3} / \text { plasma coating } \\
\text { IVDide layer between the }\end{array}$ & $\begin{array}{l}\text { The chamber is opened to atmosphere for } 20 \text { minutes then is } \\
\text { pumped down to }<1 \mathrm{mT} \text { Torr, argon and TMS fed in at } 1 \mathrm{sccm} \\
\text { each, pressure increased to } 50 \mathrm{mT} \text { Torr, glow discharge at } \sim 6 \\
\mathrm{~mA} \& 900 \mathrm{~V} \text { for } 45 \text { seconds. }\end{array}$ \\
$\begin{array}{l}\text { IVD/plasma coating } \\
\text { (No oxide layer) }\end{array}$ & $\begin{array}{l}\text { The chamber is pumped down to }<1 \mathrm{mT} \text { Torr, argon and TMS } \\
\text { fed in at } 1 \mathrm{sccm} \text { each, pressure increased to } 50 \text { mTorr, glow } \\
\text { discharge at } \sim 6 \mathrm{~mA} \& 900 \mathrm{~V} \text { for } 1 \text { minute. }\end{array}$ \\
$\begin{array}{l}\text { IVD/ } / \mathrm{Al}_{2} \mathrm{O}_{3} \\
(\mathrm{No} \text { plasma coating) }\end{array}$ & N/A \\
\hline
\end{tabular}




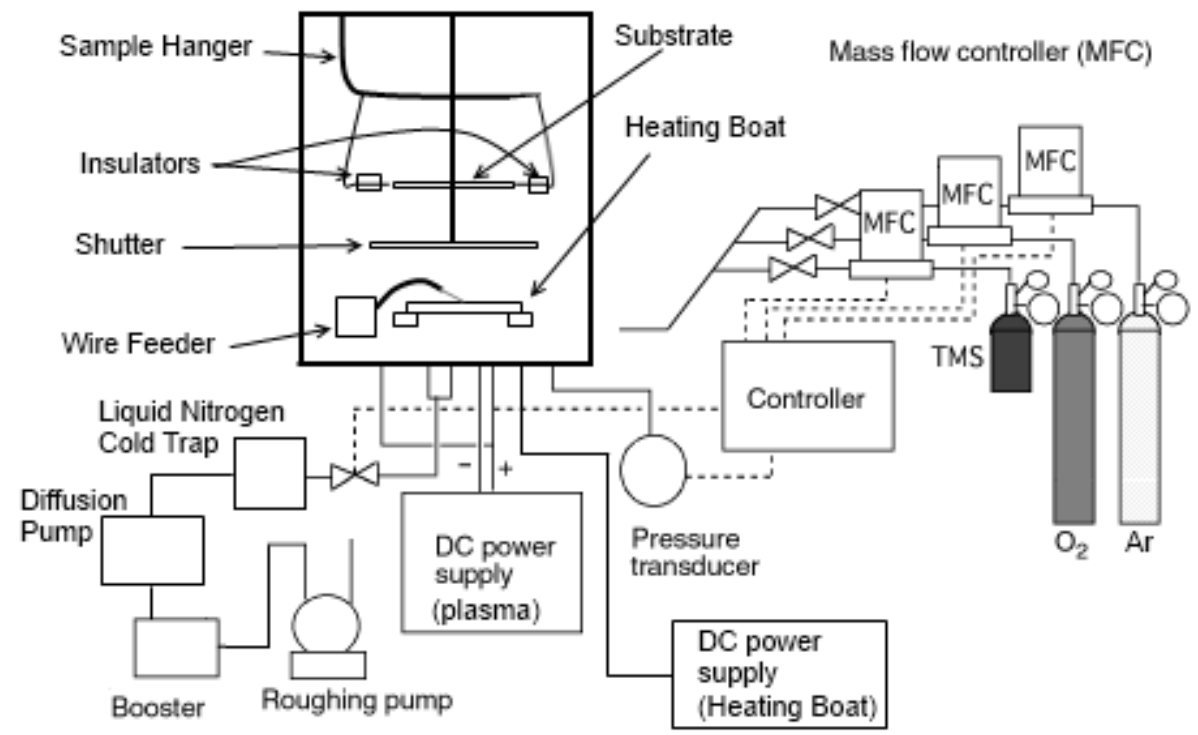

Figure 5.1. Depiction of the IVD-plasma coating hybrid reactor
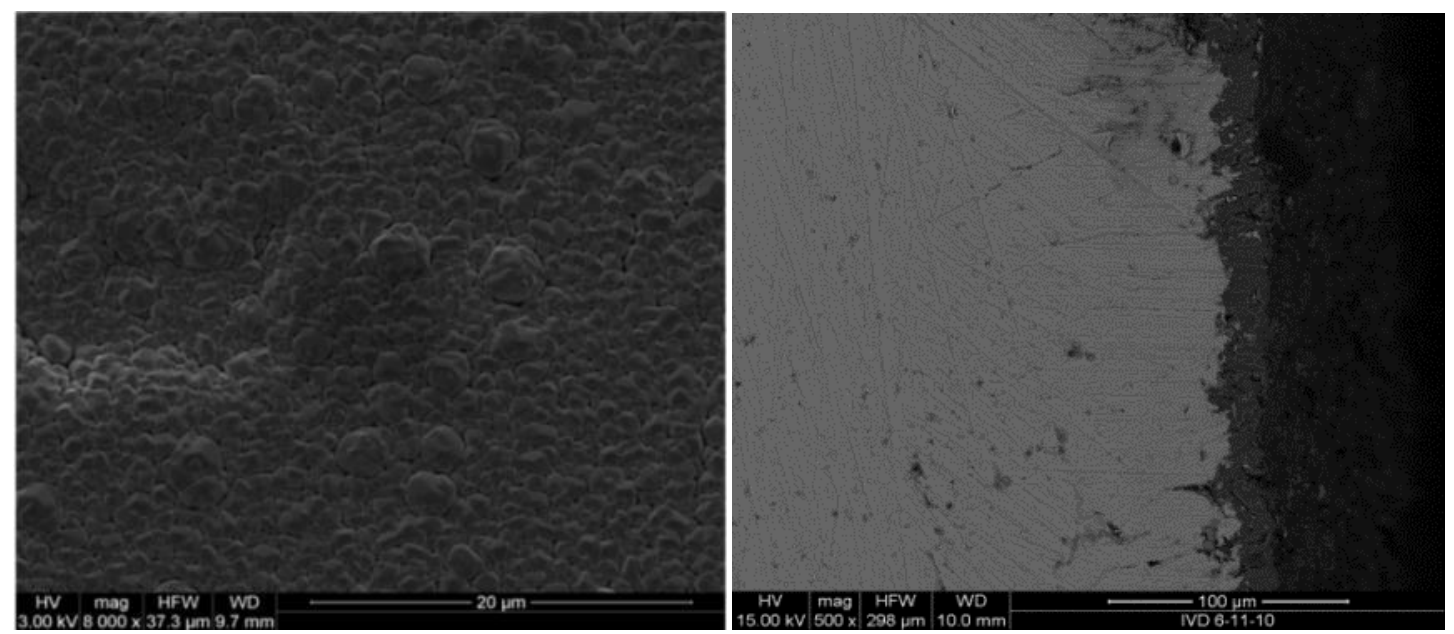

Figure. 5.2. SEM image of IVD Al coated steel, from seen from the top (left) and from the cross section (right)

\subsubsection{Corrosion Testing Samples Preparation}

The IVD Al coated panels were allowed to oxidize in atmosphere before the plasma coating was applied and therefore were named "IVD $/ \mathrm{Al}_{2} \mathrm{O}_{3} /$ plasma coating". The panels 
were coated with IVD Al coating and plasma coating in a continuous process and not allowed to form an oxide layer at the interface and therefore were named "IVD/plasma coating". The panels were IVD coated and received no plasma coating which meant an oxide layer was allowed to form when exposed to atmosphere, therefore were named "IVD/ $\mathrm{Al}_{2} \mathrm{O}_{3}$ ". A $50 \pm 2 \mathrm{~nm}$ coating deposition was found to take 45 seconds using a null-seeking type AutoEL-II Automatic Ellipsometer.

\subsubsection{Electrochemical measurements}

An EG\&G potentiostat/galvanostat (Model 273A) was used for electrochemical measurements. The potentiostat was controlled by EG\&G 352 SOFTCORR III corrosion measurement software. Measurements were carried out at room temperature in an aqueous salt solution $\left[0.5 \%\right.$ sodium chloride $(\mathrm{NaCl})+0.35 \%$ ammonium sulfate $\left(\left(\mathrm{NH}_{4}\right)_{2} \mathrm{SO}_{4}\right]$. The working electrode was the coated steel sample with a working area of 1 " $\times 1$ "; the rest of the panel surface was masked with insulating tape. The counter electrode was a graphite rod $(0.6$ $\mathrm{cm}$ in diameter); and the reference electrode was a standard silver-silver chloride $(\mathrm{Ag} / \mathrm{AgCl})$ reference electrode. All scans were taken over a twenty minute time period in the same salt solution. The scan rate for the anodic potentiodynamic polarization tests was $5 \mathrm{mV} / \mathrm{s}$. The test was terminated before passivation occurred.

\subsection{5. $\mathrm{SO}_{2}$ Salt Spray Test}

Panels were evaluated for corrosion performance at Boeing-St. Louis. $\mathrm{SO}_{2}$ salt spray corrosion resistance tests (504 hours) were performed per ASTM G85-94, annex A4. IVD Al plus TMS plasma coatings were compared with hexavalent chromate conversion coated IVD Al coated panels. Evaluation of performance was determined the appearance of corrosion products on the tested panel surfaces. 


\subsubsection{Paint Adhesion Analysis}

Five IVD coated specimens were used for evaluation of adhesion of a MIL-PRF85582, Class C2 low density primer common at Boeing-St. Louis. The primer was spray applied on IVD Al coated panels, with and without chromate conversion or TMS plasma coating in the following manner:

- 1) Bare IVD, glass bead peened before primer painting;

- 2) Bare IVD, no glass bead peen before primer painting;

- 3) TMS coated IVD, glass bead peened before primer painting;

- 4) TMS coated IVD, no glass bead peen before primer painting;

- 5) Glass bead peened IVD, chromate conversion coated before primer painting;

The primer was allowed to cure at ambient conditions for 14 days. The test method used for evaluation of primer adhesion to the substrate was defined in ASTM D 3359, Method A.

\subsubsection{XPS Analysis}

X-ray photoelectron spectroscopy (XPS) spectra were acquired using a Kratos Axis 165 Photoelectron Spectrometer, using a Mg anode at $225 \mathrm{~W}$. Survey and high resolution spectra were collected at pass energies of 80 and $20 \mathrm{eV}$ respectively and a step size of 0.5 and $0.1 \mathrm{eV}$ respectively. CasaXPS V. 2.3.15 software was used to analyze the spectra.

\subsubsection{EDS Analysis}

Energy dispersive spectroscopy (EDS) data was acquired using a FEI Quanta 600 FEG Extended Vacuum Scanning Electron Micrscope. The microscope was operated in high vacuum mode with an accelerating voltage of $3.5 \mathrm{kV}$, and 500 times magnification. Spectra were obtained with a pioneer detector and analyzed using ThermoFisher System Six V.2.0. 


\subsection{Results and Discussion}

\subsubsection{Corrosion protection}

Corrosion protection was measured using anodic potentiodynamic polarization. Anodic potentiodynamic polarization is an electrochemical technique of measuring the electrical current as a function of applied voltage for a metal immersed in a salt solution [14]. In anodic polarization, a high negative voltage is applied, driving the reduction half-cell chemical reaction, and slowly increased in the positive direction, driving reaction toward the oxidation half-cell chemical reaction. The open circuit corrosion potential $\left(\boldsymbol{E}_{\boldsymbol{C o r r}}\right)$ may be determined at the point when each half-cell reaction is in equilibrium. This is the point at which corrosion current reaches a maximum. $\boldsymbol{E}_{\text {Corr }}$ shows the difference in the number of electroactive species at the substrate and that of the reference electrode as related by the Nernst equation. In general, $\boldsymbol{E}_{\text {Corr }}$ gives insight into the susceptibility of the metal to corrosion. The corrosion current $\left(\boldsymbol{I}_{\boldsymbol{C o r r}}\right)$ is directly related to the rate of corrosion. $\boldsymbol{I}_{\boldsymbol{C o r r}}$ can be determined at $\boldsymbol{E}_{\text {Corr }}$ by the Butler-Volmer equation through interpolating the plot at the sharp peak using a tafel fit. Figure 5.3 shows anodic polarization curves of bare IVD and plasma coated IVD panels. Corrosion potentials and corrosion currents are summarized in Table 5.2. The sample "IVD/ $\mathrm{Al}_{2} \mathrm{O}_{3} /$ plasma coating" had the lowest corrosion current. Both TMS coated samples, with and without an oxide interface, outperformed bare IVD, i.e. "IVD $/ \mathrm{Al}_{2} \mathrm{O}_{3}$," based on corrosion current. 


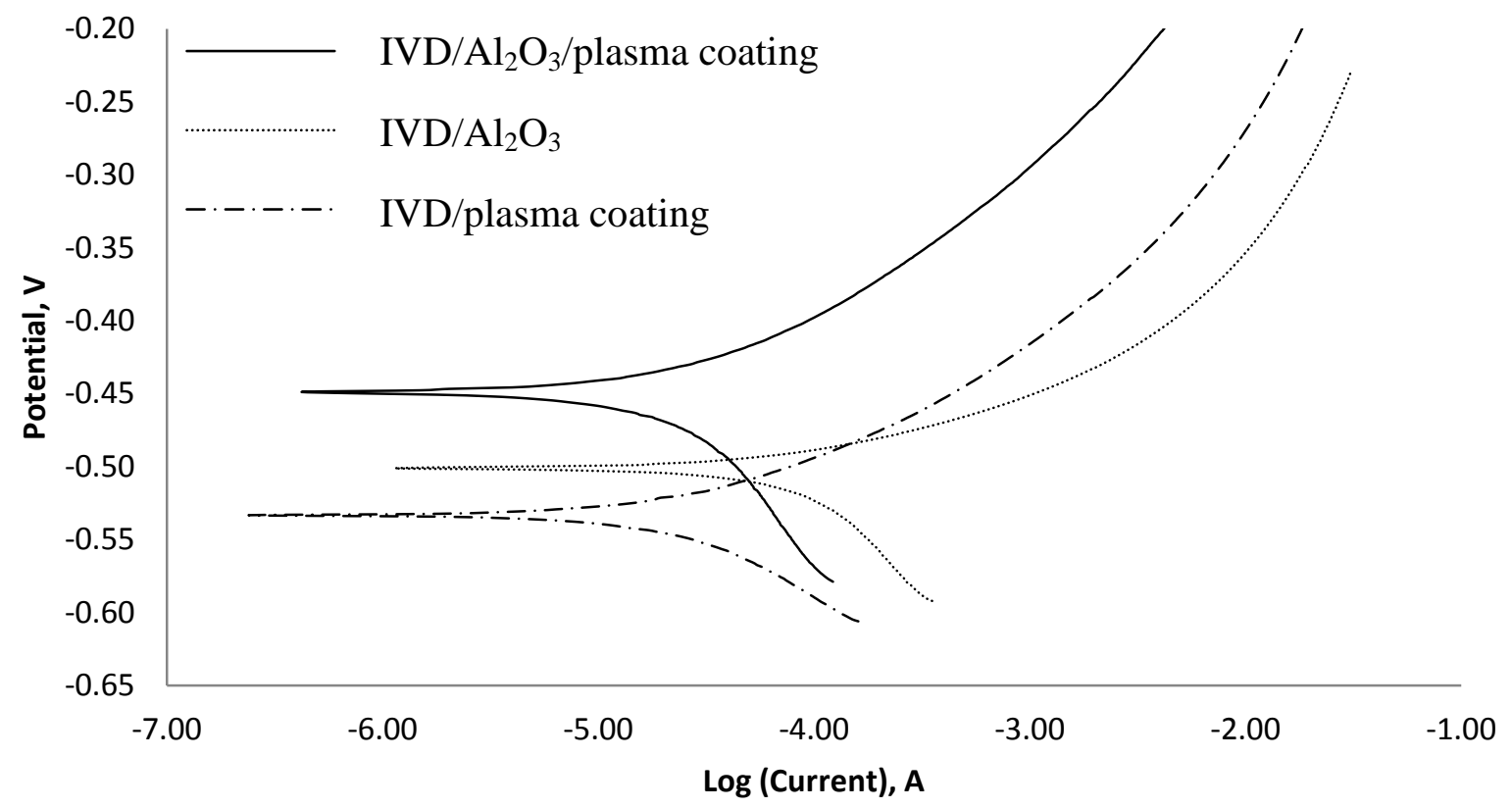

Figure 5.3. Typical anodic polarization curves of IVD Al coated steel with and without various plasma coating.

Table 5.2. Electrochemical measurements of IVD coated steel with and without various plasma coating based on anodic polarization.

\begin{tabular}{lll}
\hline Sample & $\boldsymbol{I}_{\text {Corr }}(\mathbf{A})$ & $\boldsymbol{E}_{\text {Corr }}(\mathbf{m V})$ \\
\hline $\mathrm{IVD} / \mathrm{Al}_{2} \mathrm{O}_{3} /$ plasma coating & $2.571 \mathrm{e}-5 \pm 3.083 \mathrm{e}-6$ & $-454.8 \pm 2.053$ \\
$\mathrm{IVD} /$ plasma coating & $3.437 \mathrm{e}-5 \pm 5.671 \mathrm{e}-6$ & $-524.2 \pm 12.547$ \\
$\mathrm{IVD} / \mathrm{Al}_{2} \mathrm{O}_{3}$ & $8.032 \mathrm{e}-5 \pm 5.629 \mathrm{e}-6$ & $-501.4 \pm 1.145$ \\
\hline
\end{tabular}

The IVD panels were next evaluated by neutral salt fog testing after 504 hours as per standard Boeing protocol and the results were compared with chromate coated IVD. Plasma coating is performed with IVD coated panels allowed to form an oxide layer. A "pass" grade is given to panels in which red corrosion byproducts are not visible. Figure 5.4, a) and b), showed IVD coated panels coated with the hybrid IVD/plasma coater received the passing 
grade. A similar amount of corrosion byproducts are created on the chromate conversion coated panel as with the TMS coated panel after 504 hours neutral salt fog testing. This is strong evidence that TMS plasma coating offers a similar corrosion protection to chromate coating. The corrosion products seem to be greater than Boeing produced IVD shown in Figure $5.4 \mathrm{c}$ ). The panel coated by Boeing was glass bead peened as a standard step in Boeing's production process. Also, the microstructure of panels coated by the hybrid coater differed slightly from that of Boeing as observed by SEM in Figure 5.5. UMC produced IVD has a slightly smaller nodular structure. Because of the difference in IVD structure, a fourth panel was IVD coated by UMC without any further treatments and evaluated after 336 hours testing to evaluate the corrosion improvement made by plasma coating. Figure 5.4 d) shows the bare IVD panel would also receive a grade of "passing", but a slight red discoloration did start due to corrosion of underlying steel. Comparing Figure 5.4 b) with Figure 5.4 d), the corrosion resistance afforded by TMS plasma coating is apparent.
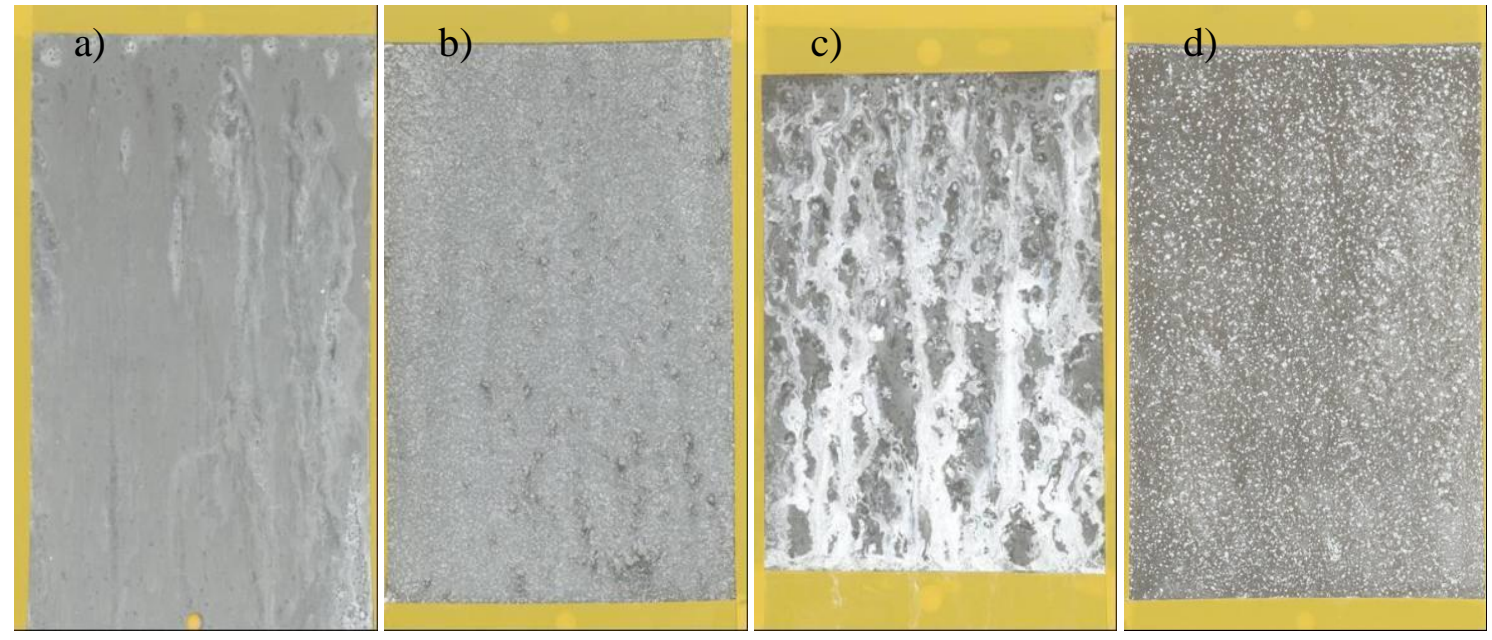

Figure 5.4. Typical images of IVD coated panels after neutral salt fog testing; a) with chromate conversion coating after 504 hours, b) with TMS plasma coating after 504; c) Boeing produced IVD with chromate conversion coating after 504 hours; d) without further coating after 336 hours. 

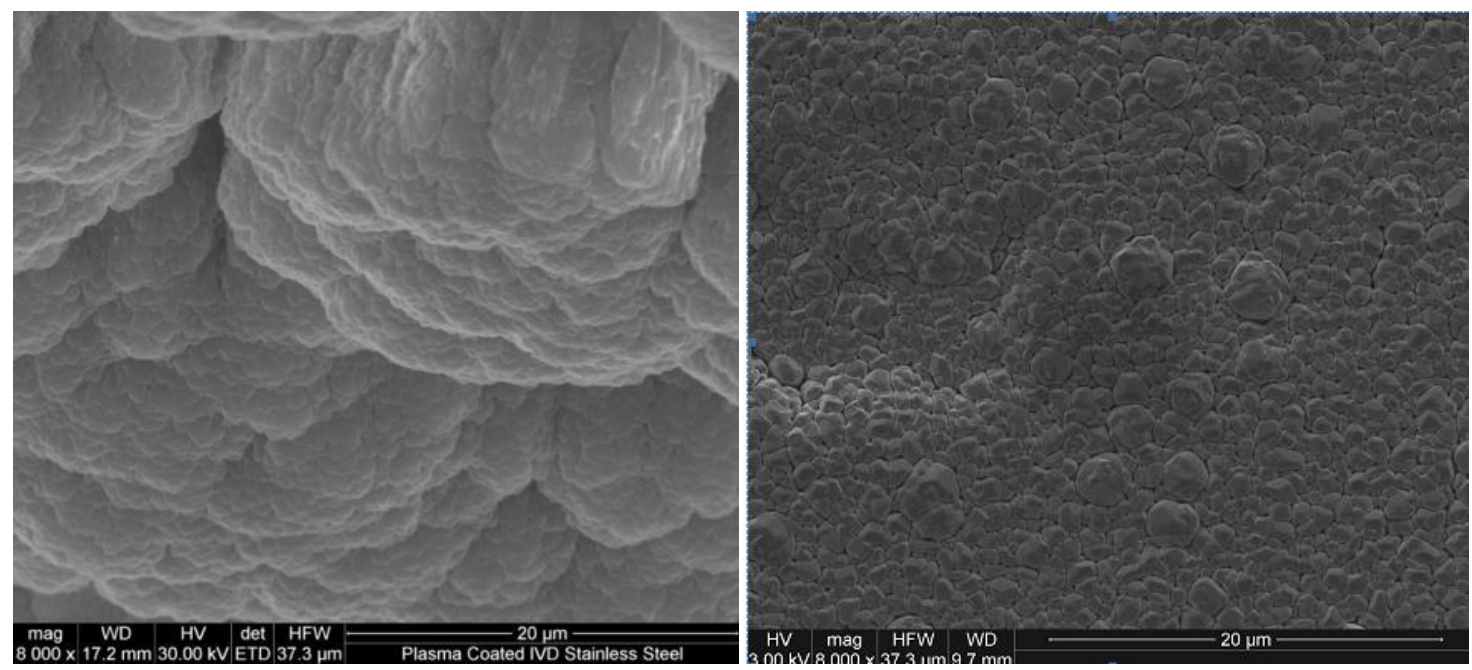

Figure 5.5. SEM images of IVD coated panels; produce by Boeing (left) and hybrid reactor (right).

\subsubsection{Paint Adhesion}

All TMS plasma coated panels passed primer adhesion testing MIL-PRF-85582 Class C2 per ASTM D 3359 (images not shown) with a score of 5A. TMS plasma coating had a passing grade even with the coating being glass bead peened before primer paint was applied to demonstrate the efficacy of the plasma coating adhesion.

\subsubsection{Evaluation of Possible Contamination}

There was concern that producing TMS plasma coating in the same reactor as the IVD coater might cause cross-contamination of the chamber and therefore contamination of subsequent IVD Al coatings. No contamination of the chamber was visually apparent after TMS plasma coating. To quantitatively check for contamination of subsequent IVD, EDS was performed to measure the chemical composition before and after the plasma coating trials, as shown in Table 5.4. Bare IVD Al produced prior to plasma coating experiments showed the same amount of carbon compared to bare IVD Al produced after plasma coating experiments. There was slight discoloration of substrate holders after TMS plasma coating 
experiments, but this was effectively removed by oxygen plasma cleaning. It was intentional to coat all IVD only specimens first, and then followed with the IVD and TMS plasma coated panels to avoid contamination, i.e. detrimental or beneficial performance effected by the use of TMS in the common coater. Figure 5.6, shows a corrosion resistance panel that is a Class 2 IVD Al coating that passed adhesion testing by glass bead peen, i.e. IVD did not delaminate after glass bead peening. The panel was then chromate conversion coated and subjected to 5\% neutral salt fog for 504 hours. The coating passed IVD coating adhesion testing and corrosion resistance testing and would seem to demonstrate that IVD Al may be applied in a coater that is also used for TMS plasma coating, with no detrimental effects on the IVD coating. 


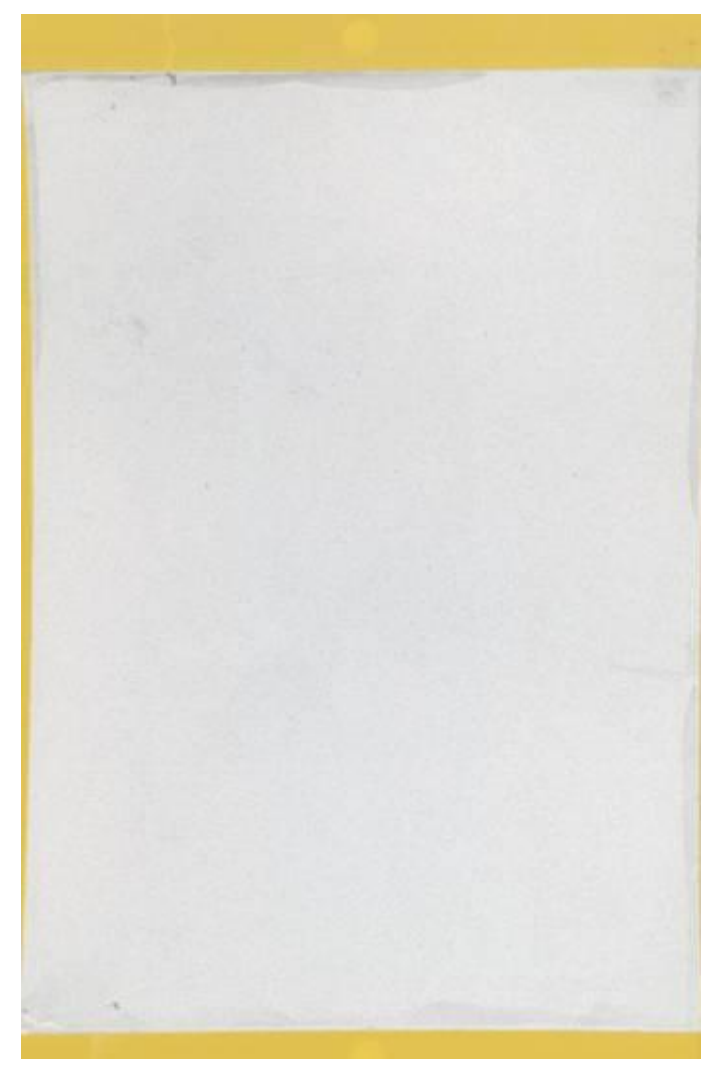

Figure 5.6. Typical image of IVD coated panel with coating applied by hybrid coater following completion of all plasma coating trials, Corrosion Resistance Test-504 Hours Neutral Salt Fog.

\subsubsection{Plasma Nanocoating Characterization}

X-ray photoelectron spectroscopy (XPS) was performed to characterize the surface composition of the coatings with and without oxide at the IVD-TMS interface, as shown in Figure 5.7 and summarized in Table 5.3. XPS does not penetrate to the IVD-TMS interface, which is evident from the low aluminum concentration. Although XPS does not reach the IVD-TMS interface, the spectra indicate that the sample with an oxide interface has a much higher oxygen concentration in the TMS coating than the sample with no oxide interface. The natural oxide layer formed at the interface must be sputtered into the reactor chamber and joined in the plasma nanocoating resulting in a higher oxygen content. XPS shows the 
sample without oxygen treatment at the IVD $\mathrm{Al}$ interface, i.e. the "IVD/plasma coating" sample, has a substantially lower oxygen concentration. TMS plasma coatings were inevitably oxidized when removed from vacuum and exposed to air. Some residual free radicals remained on plasma coatings can be quenched and oxidized rapidly in atmosphere and further oxidation proceeds then after. Gengenbach et al. have shown plasma polymer XPS spectra shifting of the O1S peak by around $0.3-0.4 \mathrm{eV}$ over a period of the first 10 days of ageing in air [15]. The plasma nanocoating is composed of many chemical groups causing a broadening of XPS spectra. As seen in Figures 5.8-5.10, the shift in high resolution peaks is consistent among $\mathrm{O}$ 1s, Si 2p, and C 1s peaks, with "IVD/ $\mathrm{Al}_{2} \mathrm{O}_{3} /$ plasma coating" and "IVD/ plasma coating" respectively. The difference in binding energy between the natural oxide layer sample, i.e. "IVD/ $/ \mathrm{Al}_{2} \mathrm{O}_{3} /$ plasma coating" and the sample without oxide layer, i.e. "IVD/plasma coating", is around $1 \mathrm{eV}$ or greater for each mentioned chemical peak. Fonseca et al. found that tetramethylsilane plasma polymers consisted mainly of a cross-linked $\left[\mathrm{CH}_{3}\right]_{\mathrm{n}} \mathrm{SiH}_{\mathrm{m}}-$ structure [16]. They also found the $\mathrm{Si} 2 \mathrm{p}$ peak centered around $100.7 \mathrm{eV}$, indicative of Si-H and Si-C bonding and a shift to $101.2 \mathrm{eV}$ after one month oxidation likely due to $\mathrm{Si}-\mathrm{O}$ and $\mathrm{O}-\mathrm{Si}-\mathrm{O}$ bonding. Likewise, they found the $\mathrm{C} 1$ s bonding centered around $285.0 \mathrm{eV}$, indicative of $\mathrm{C}-\mathrm{C}$ and $\mathrm{C}-\mathrm{Si}$ bonding and a shift to $\mathrm{C}-\mathrm{O}(286.6 \mathrm{eV})$ and $\mathrm{C}=\mathrm{O}(287.9$ eV) functionalities after oxidation. Our study revealed a similar shifting between the sample with a natural oxide layer at the interface versus the sample without an interfacial oxide layer. The shifts were Si $2 p$ spectra from $\sim 100.0 \mathrm{eV}$ to $\sim 101.7 \mathrm{eV}$ and a shift from $\sim 283.4 \mathrm{eV}$ to $\sim 284.3 \mathrm{eV}$ of the $\mathrm{C} 1 \mathrm{~s}$ spectra, IVD/TMS and IVD/NO/TMS respectively. Oxidation of the coating causes a Si $2 p$ and C 1s peak shift to higher binding energy. Grundmeier et al. found that, in thin microwave organosilicon plasma polymers, carbon was mainly bonded with 
silicon while oxygen selectively bound with silicon [6]. Our data suggested a similar trend: oxygen bonding preferentially with silicon caused the aforementioned $\sim 1.7 \mathrm{eV}$ shift in the $\mathrm{Si}$ $2 p$ peak while only shifting the $\mathrm{C} 1 \mathrm{~s}$ peak by $\sim 0.9 \mathrm{eV}$. The peak shift in XPS spectra is due to oxygen being very electronegative and therefore attracting the electron cloud towards itself. When plasma coating is performed in a continuous flow reactor, Si-containing species have been found to deposit at a similar rate to C- containing species [10]. During the plasma coating of the "IVD/ $/ \mathrm{Al}_{2} \mathrm{O}_{3} /$ plasma coating" sample the oxygen content in the plasma will be the highest at the beginning of the plasma coating process due to the high concentration of oxygen on the aluminum surface. The high oxygen content in the "IVD/ $\mathrm{Al}_{2} \mathrm{O}_{3} / \mathrm{plasma}$ coating" sample, as observed in Figure 5.8, causes the formation of $\mathrm{CO}$ or $\mathrm{CO}_{2}$ which may be pumped out of the reactor system. This should result in a high silicon concentration near the interface with a gradual change to a higher carbon concentration near the plasma coating exterior surface as oxygen in the plasma becomes scarce.

Aluminum surfaces develop a dense passivation film of aluminum oxide in a few seconds after exposure to ambient air [17]. This passivation film is a major source of corrosion protection, so it was expected that the plasma coated sample with an oxide layer at the IVD-TMS interface should have the best corrosion protection. Even without a substantial passivation layer, the "IVD/plasma coating" sample had a similarly low corrosion current due to the barrier plasma coating. The magnitude of corrosion potential was highest for the "IVD/plasma coating" sample. It should be expected that a higher $\boldsymbol{I}_{\text {Corr }}$ value should correspond with the higher $\boldsymbol{E}_{\text {Corr }}$ value. The more negative $\boldsymbol{E}_{\text {Corr }}$ could be due to the absence of $\mathrm{Al}_{2} \mathrm{O}_{3}$ at the IVD interface resulting in less passivation and due to less covalent bonding between TMS plasma coating and IVD Al without oxygen as a bridge between $\mathrm{Si}$ and $\mathrm{Al}$ 
elements. Our XPS data could not provide the information about bonding at the IVD-TMS plasma coating interface due to the limit of detection depth by this surface chemistry analytical method, but some predictions can be made. TMS containing both Si and C elements can bond with aluminum in four ways: Si-Al, C-Al, Si-O-Al, or C-O-Al. Carbon can bond with aluminum, such as in the molecule trimethylaluminum, but the $\mathrm{C}$-Al bond alone is unstable [18]. Indeed, molecules like trimethylaluminum require a three center two electron agostic bond to be stable. Even still, the bond strength is low, less than $40 \mathrm{~kJ} / \mathrm{mol}$ [19]. Oxygen must bridge between carbon and aluminum to form strong adhesion between the plasma nanocoating and IVD Al [20].

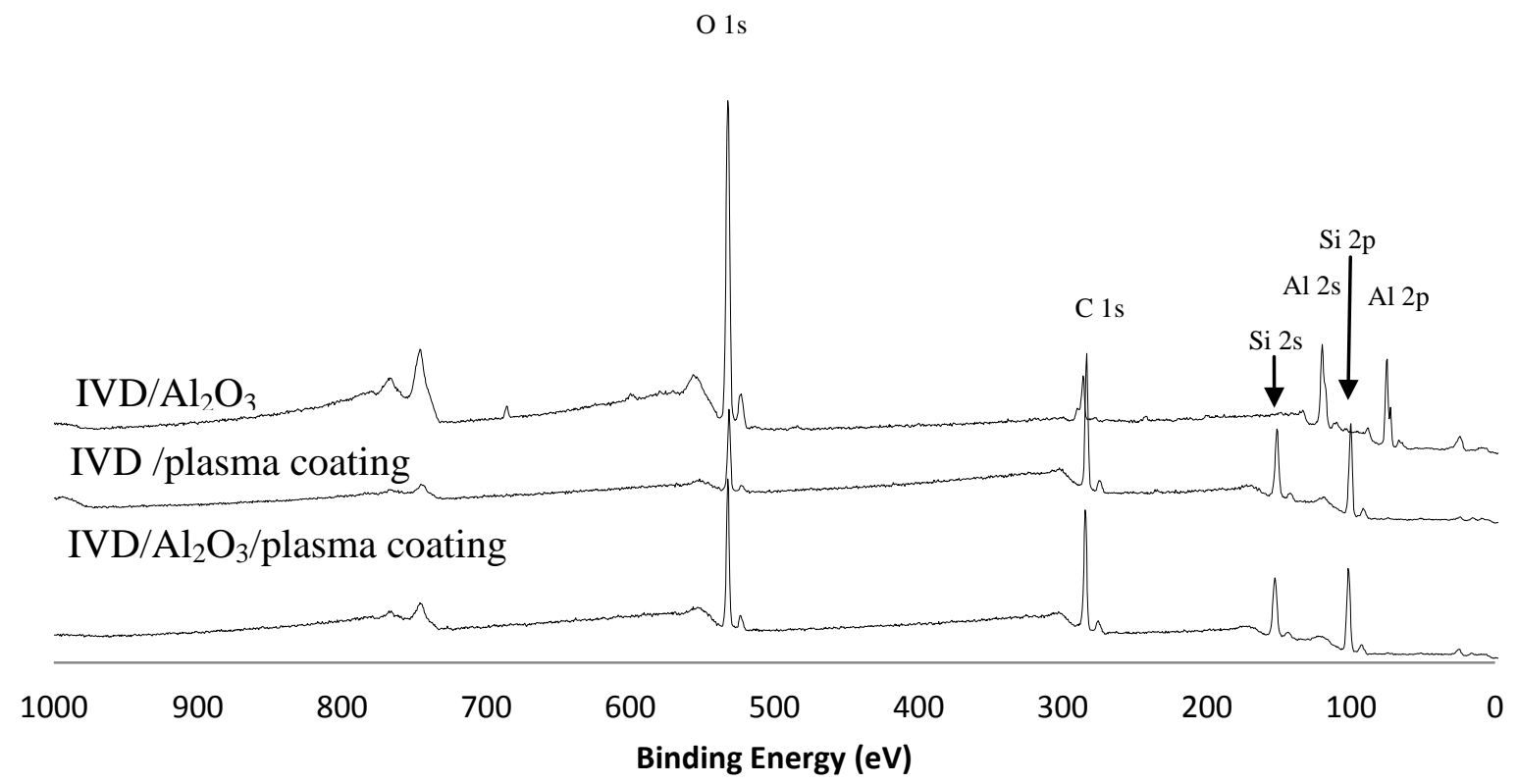

Figure 5.7. XPS spectra taken from the top surface of IVD coated steel with and without various plasma coating. 
Table 5.3. Atomic concentration percentage of IVD coated steel with and without various plasma coating obtained by XPS analysis.

\begin{tabular}{lllll}
\hline Sample & Al & O & C & Si \\
\hline IVD $/ \mathrm{Al}_{2} \mathrm{O}_{3}$ /plasma coating & 0.29 & 17.54 & 41.12 & 41.05 \\
IVD $/ \mathrm{plasma} \mathrm{coating}_{\text {IVD } / \mathrm{Al}_{2} \mathrm{O}_{3}}^{0.71}$ & 9.52 & 46.82 & 42.94 \\
\hline
\end{tabular}

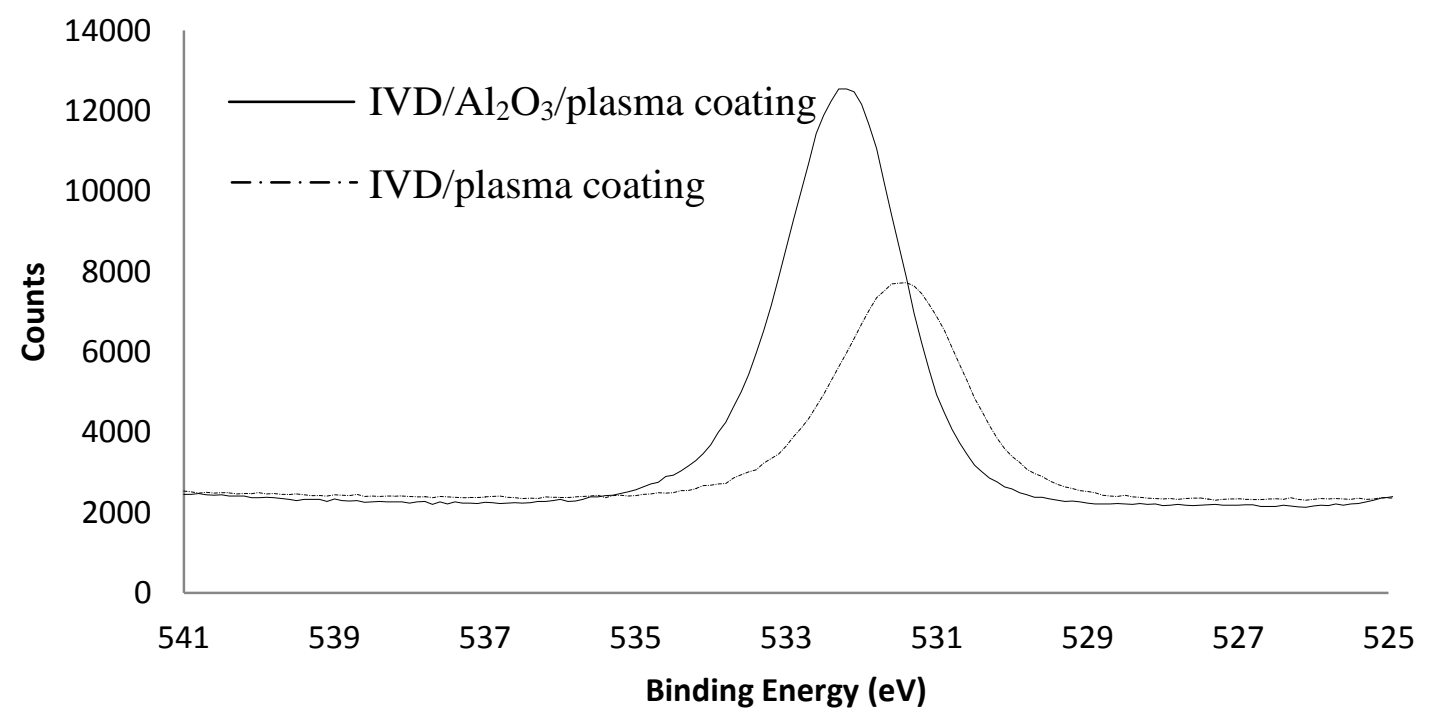

Figure 5.8. High resolution XPS peak analysis of O 1s peaks from the top surface of IVD coated steel with plasma coating.

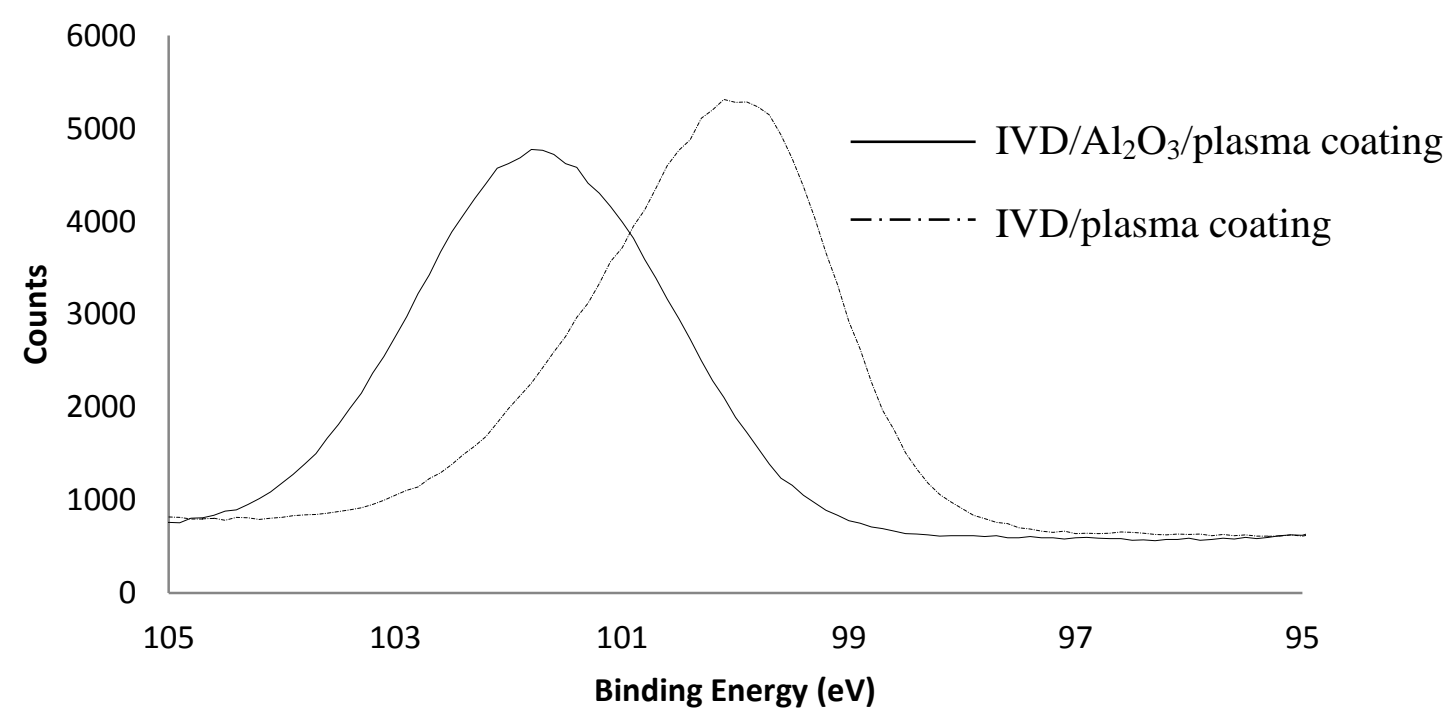


Figure 5.9. High resolution XPS peak analysis of Si 2p peaks from the top surface of IVD coated steel with plasma coating.

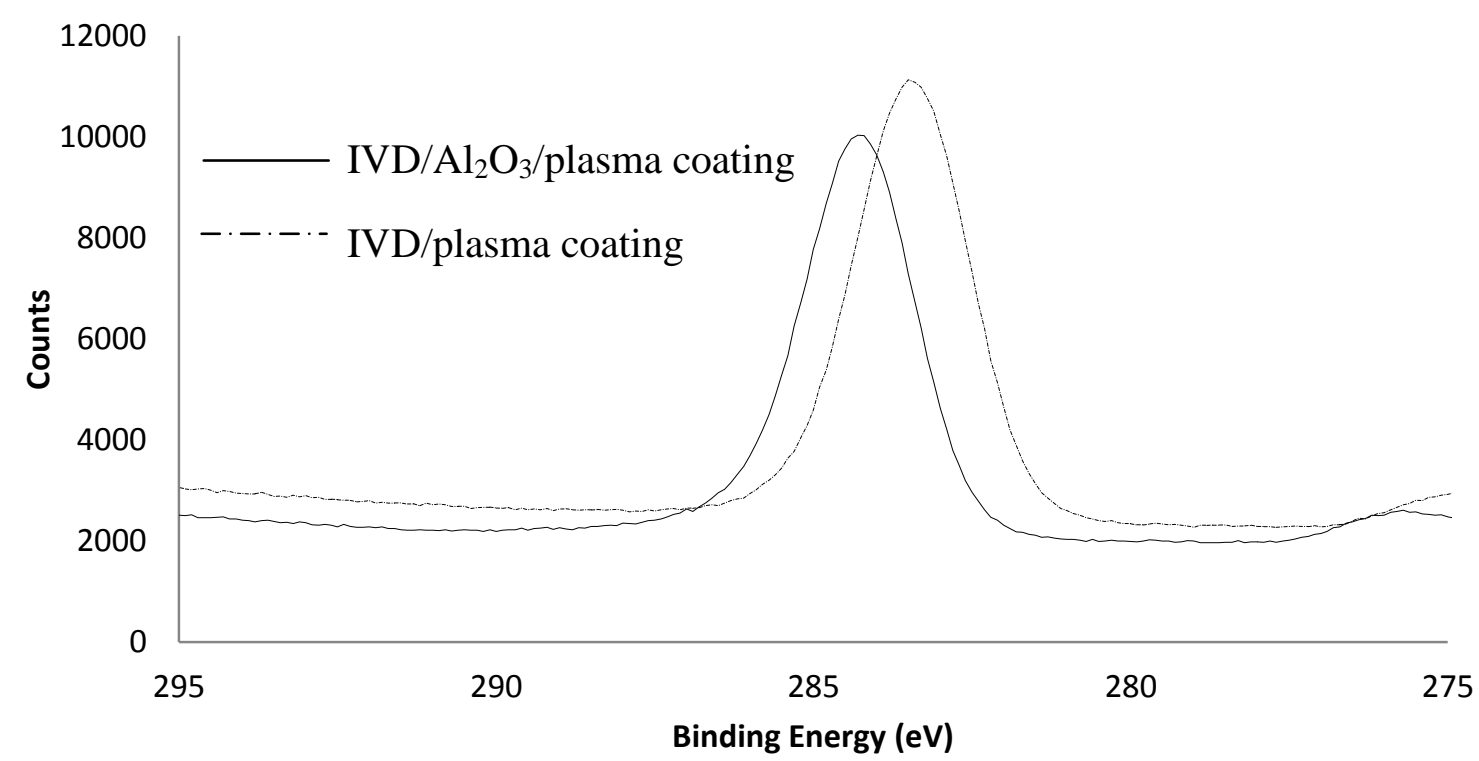

Figure 5.10. High resolution XPS peak analysis of C 1s peaks from the top surface of IVD coated steel with plasma coating.

Corrosion is a complicated process; various corroding mechanisms may require a finite amount of time to be activated. Diffusion of water through a coating is inevitable and may require minutes to days to occur depending on the permeability and surface energy of the coating. Once water has diffused to the metal surface, corrosion may occur and delaminate the coating from the substrate causing increased corrosion. Also, intrusion of hydrated salt ions may take longer to occur due to their larger size and charge [21]. Anodic polarization was performed in a short time period and therefore cannot evaluate the long term performance of a coating to long term diffusing water and hydrated salt ions. Further, delamination of the coatings caused by corrosion byproducts also requires a long term evaluation. This shows the need for long term 504 hour salt spray testing. The addition of $\mathrm{SO}_{2}$ into the test can further accelerate corrosion by the dissolution of the passivating 
aluminum oxide layer. A $13 \mu \mathrm{m}$ thick coating is considered a class 2 coating and therefore only requires a passing grade after 336 hours, yet our coating received a passing grade for the 504 hour test which is the standard for the thicker class $1(25 \mu \mathrm{m}$ thickness $)$ coatings. Our results showed that TMS plasma nanocoating was able to impede intruding salt ions and maintained strong water insensitive adhesion to the IVD Al coated surfaces.

\subsection{Conclusion}

Both TMS plasma coating and IVD Al coating were deposited on to steel substrates using a hybrid IVD/plasma coater. The effect of the oxide layer at the IVD-TMS interface was evaluated. TMS plasma coated on IVD $\mathrm{Al}$ with and without an $\mathrm{Al}_{2} \mathrm{O}_{3}$ layer at the IVD surface both showed a decrease in $\boldsymbol{I}_{\text {Corr }}$ as compared to bare IVD. TMS plasma coating effectively impeded the transport of water and hydrated salt ions to the IVD surface causing a reduced corrosion current. TMS plasma coating without an $\mathrm{Al}_{2} \mathrm{O}_{3}$ layer at the IVD-TMS interface did not see an improvement over bare IVD with respect to $\boldsymbol{E}_{\boldsymbol{C o r r}}$ due to: the low electrical conductivity of the TMS plasma coating, the reduced amount of bonding with the surface due to the lack of an oxygen bridge, and the lack of an $\mathrm{Al}_{2} \mathrm{O}_{3}$ passivation layer. Therefore, it is recommended the IVD Al be allowed to form a natural oxide layer before TMS plasma coating. The current industrial IVD Al coater, i.e. the Ividizer, is routinely opened to atmosphere after IVD Al coating. The incorporation of TMS plasma coating with a naturally occurring oxide layer should not cause any problems with the Ividizer. The ability to apply TMS plasma coating to IVD Al coated samples that have been exposed to atmosphere for days may be a useful option. The incorporation of the plasma coater with an IVD coater will reduce cost due to less equipment especially when compared to the costs of 
the electroplating and baking processes of chromate conversion coating and the resulting waste water treatments. The formation of a natural oxide layer would therefore be inherent in that process. For the feasibility study, the corrosion resistance results for all specimens pass the requirement for a chromate treated, or Type II IVD Al coating. Incorporation of plasma coating into the IVD reactor did not cause contamination from the plasma coating process to subsequent IVD trials. The two processes do not affect each other as shown by corrosion testing and chemical composition analysis. Therefore it should be considered safe to combine the processes into one reactor chamber for improved efficiency and costeffectiveness.

\section{Acknowledgements}

The authors would like to thank The Boeing Company, particularly Bruce Griffin, for managing this work, the United States Air Force (USAF) for funding the research, Mike Bridger for help with IVD setup, the University of Missouri's EMC core, Missouri University of Science and Technology Material's Research Center, and Dr. Anthony Caruso's group at the University of Missouri-Kansas City for XPS analysis. 


\subsection{References}

1. Mattox, D., 2000, "Ion plating-past, present and future," Surface and Coatings Technology, 133-134, 517-521.

2. EPA Federal Register, National Emission Standards for Hazardous Air Pollutants for Source Categories: Aerospace Manufacturing and Rework Facilities, Vol. 60 (170), September 1995, p. 45947.

3. Chan, Y., Qingsong, Y., 2005, "Electrochemical characterization of plasma polymer coatings in corrosion protection of aluminum alloys," J. Vac. Sci. Technol. A, 23, 991-997.

4. Yasuda, H.K., Yu, Q. S., 2001, "Deposition of trimethylsilane in glow discharges," J. Vac. Sci. Technol. A, 19, 773-781.

5. Yu, Q., Yasuda, H., 2005, "Protection of aluminum alloys from filiform corrosion by low-temperature plasma interface engineering," Progress in Organic Coatings, 52, 217-226.

6. Grundmeier, G., Thiemann, P., Carpentier, J., Shirtcliffe, N., Stratmann, M., 2004, "Tailoring of the morphology and chemical composition of thin organosilane microwave plasma polymer layers on metal substrates," Thin Solid Films, 446, 61-71.

7. Reddy, C.M., et al., 2000, "Improved corrosion protection of aluminum alloys by system approach interface engineering: part 1-Alclad 2024-T3," Corrosion, 56, 819831.

8. Iriyama, Y., Ihara, T., Kiboku, M., 1996, "Plasma polymerization of tetraethoxysilane on alumnum granule for corrosion protection," Thin Solid Films, 287, 169-173.

9. Taylor, C.E., Boerio, F.J., 1999, "Plasma Polymer Films as Adhesion Promoting Primers for Aluminum Substrates. Part 1: Characterization of Films and Film/Substrate Interfaces," J. Adhesion, 69, 217-236.

10. Yu, Q., Moffitt, C.E., Wieliczka, D.M., Deffeyes, J., Yasuda, H., 2002, “Corrosion protection of ion vapor deposition (IVD) Al-coated Al alloys by low-temperature plasma interface engineering Part III-DC cathodic polymerization in a closed reactor system," Organic Coatings, 44, 37-47.

11. Grundmeier, G., Thiemann, P., Carpentier, J., Barranco, V., 2003, "Tailored thin plasma polymers for the corrosion protection of metals," Surface and Coatings Technology, 174-175, 996-1001. 
12. Yasuda, H.K., Yu, Q.S., Reddy, C.M., Moffitt, C.E., Wieliczka, D.M., 2002, "Adhesion of the Plasma Polymer of Trimethylsilane to Aluminum Alloys," Journal of Applied Polymer Science, 85, 1387-1398.

13. Batan, A., et al., 2010, "Evidence of covalent bond formation at the silane-metal interface during plasma polymerization of bis- 1,2-(triethoxysilyl)ethane (BTSE) on aluminum," Chemical Physics Letters, 493, 107-112.

14. Zhang, X., Jiang, Z., Yao, Z., Song, Y., Wu, Z., 2009, "Effects of scan rate on the potentiodynamic polarization curve obtained to determine the Tafel slopes and corrosion current density," Corrosion Science, 51, 581-587.

15. Gengenbach, T., Chatelier, R., Griesser, H., 1996, "Correlation of the Nitrogen 1s and Oxygen 1s XPS Binding Energies with Compositional Changes During Oxidation of Ethylene Diamine Plasma Polymers," Surface and Interface Analysis, 24, 611-619.

16. Fonseca, J., Apperley, D., Badyal, J., 1993, "Plasma polymerization of tetramethylsilane," Chemistry of Materials, 5, 1676-1682.

17. Kuznetsova, A., Yates, J.T., 2001, "Making a Superior Oxide Corrosion Passivation Layer on Aluminum Using Ozone," Langmuir, 17, 2146-2152.

18. Weast, R.C., 1989, CRC Handbook of Chemistry and Physics, CRC Press, Boca Raton, FL.

19. Von Frantzius, G., Streubel, R., Brandhorst, K., Grunenberg, J., 2006, "How Strong is an Agostic Bond" Direct Assessment of Agostic Interactions Using the Generalized Compliance Matrix," Organometallics, 25, 118-121.

20. Bichler, C.H., Langowski, H.C., Moosheimer, U., Seifert, B., 1997, "Adhesion mechanism of aluminum, aluminum oxide, and silicon oxide on biaxially oriented polypropylene (BOPP), poly(ethyleneterephthalate) (PET), and poly(vinyl chloride) (PVC), J. Adhesion Sci. Technol., 2, 233-246.

21. Chen, M., Yu, Q., Reddy, C.M., Yasuda, H.K., 2000, "Model Study Investigating the Role of Interfacial Factors in Electrochemical Impedance Spectroscopy Measurements," Corrosion, 56, 709-721. 


\title{
Chapter 6 Plasma Sterilization of Oral Bacteria
}

\begin{abstract}
Objective: To study the deactivation effects and mechanisms of atmospheric pressure plasma treatment of common oral bacteria.

Methods: An atmospheric pressure argon plasma brush was used to deactivate two different Gram-positive oral bacteria with varied additions of oxygen, cell concentrations, and plasma exposure times. The oral bacteria Lactobacillus acidophilus and Streptococcus mutans were seeded on hydroxyapatite supporting media and their survivability was studied. SEM was performed to examine bacterial morphological changes.

Results: The experimental data indicated that a plasma exposure time of 13 seconds effectively killed all bacteria when concentrations were less than $6.9 \times 10^{6} \mathrm{cfu} / \mathrm{cm}^{2}$ for $L$. acidophilus and $1.7 \times 10^{7} \mathrm{cfu} / \mathrm{cm}^{2}$ for $S$. mutans. At higher bacterial concentrations of $1.2 \times 10^{8}-2.5 \times 10^{8} \mathrm{cfu} / \mathrm{cm}^{2}$, a $1.5 \log$ reduction in bacterial concentration was observed in the first 7 seconds, but nearly no further reduction in bacterial population was achieved after 20 seconds. SEM showed plasma deactivation occurred mainly on the top surface of the bacteria supporting medium, while shadowing effects from the resulting bacterial debris reduced plasma deactivation of the underlying bacteria.

Conclusions: The results indicate the plasma brush was fast and effective in deactivation of top surface bacteria mainly through bombardment of charged and neutral plasma species.
\end{abstract}




\subsection{Introduction}

It is well known that oral bacteria are the cause of dental caries [1]. Oral bacteria excrete acids during their fermentation of residual food particles in the mouth. The acid dissolves the carbonated hydroxyapatite which is a major component of tooth enamel and dentin. During caries restoration, sclerotic tissue is removed by drilling. There is no clear line where sclerotic tissue ends and healthy tissue begin; thus, it is standard practice to remove a good portion of healthy tissue to ensure total removal of bacteria. ${ }^{2}$ In order to conserve as much healthy tissue as possible, a fast and effective method for deactivation of oral bacteria during caries restoration is needed. Currently, caries prevention is based on disinfecting chemicals, such as peroxides and antibiotics, in pastes and oral rinses, and by physical agitation and removal by brushes, dental picks, water jets, and other tools. These methods require reoccurring treatments, but caries prevention is still not be guaranteed. Therefore, supplemental treatment methods for caries prevention should be explored. Plasma deactivation of oral bacterium is an emerging application for low temperature atmospheric plasmas [3-9]. Plasmas are capable of creating reactive chemical species through dissociation and ionization of molecules. An advantage of plasma chemistry compared to other wet chemistry is that the reactive species created in plasma are short lived and therefore they are inherently non-toxic. In comparison with wet chemical methods, plasma based methods produce very little effluent reactive chemicals. A direct current atmospheric plasma brush, capable of creating a plasma plume with an operating temperature near body temperature was developed at the University of Missouri [10].

Atmospheric plasmas, operated at temperatures safe for use in the human body, are achievable due to their non-equilibrium nature. In non-equilibrium plasmas, large molecules 
comprising the carrier gas remain at low temperatures while electrons are preferentially accelerated. The various excited states and chemical species occurring in a plasma are determined by the electron energy distribution. The energy distribution of non-equilibrium plasmas is non-Maxwellian, making it difficult to ascertain what chemical species are present, in what spatiotemporal distributions in the plasma plume, and what the interaction with the substrate will be [11]. The underlying physics behind atmospheric plasmas is highly complex; further discussion may be found elsewhere [12-13].

Most researchers suggest reactive oxygen species as a major contributor to bacteria deactivation. These include $\mathrm{OH}, \mathrm{O}, \mathrm{H}_{2} \mathrm{O}_{2}, \mathrm{O}_{2}{ }^{-}, \mathrm{NO}, \mathrm{NO}_{2}$, and $\mathrm{O}_{3}$ [4,14-15]. The exact contribution of each of these chemical species in deactivation remains somewhat unknown. This problem arises from the difficulty in separating the different species and in the measurement of their exact concentrations [14]. A review of literature gives some similar and contrary findings about the major causes of deactivation. Dobrynin et al (2009), suggests that $\mathrm{O}_{2}^{-}$is the main source of deactivation and that ozone, hydrogen peroxide, $\mathrm{OH}^{-}$, and $\mathrm{UV}$ were not major contributors to deactivation [15]. Perni et al. (2007) found OH, NO, UV to be negligible and that $\mathrm{O}_{2}{ }^{-}$and monatomic oxygen were the major contributors to plasma deactivation [16]. Similarly, a review on the subject by Gaunt found that contribution from UV was negligible, and that superoxide was a major contributor [17]. In Boudam's review on plasma deactivation, UV light was found to be either negligible or significant on deactivation depending on experimental setup [18]. Similar to Perni (2007), Goree et al. (2006) found monatomic oxygen to be a major contributor to deactivation, but contrary to Perni, they found $\mathrm{OH}$ to be a major contributor [4]. Due to the lack of consensus, in this research, particular emphasis has been made on studying the role of reactive of oxygen species. 
Lactobacillus acidophilus and Streptococcus mutans are common Gram-positive oral bacteria responsible for producing oral caries [19-20]. In a previous study performed by our research group, atmospheric plasma was found to be effective in deactivating bacterium when seeded on glass, filter paper, and PTFE supporting media [6]. This research is an extension of that research to a more clinically relevant supporting medium, i.e., bacterial deactivation is performed on sintered hydroxyapatite (HA) discs. HA has been used as a tooth enamel analogue in simulated dental pellicle and biofilm formation studies [21-23]. We chose to perform testing on HA discs rather than enamel due to HA's large availability and consistent uniform structure.

\subsection{Materials and Methods}

\subsubsection{Materials and Bacterium preparation}

L. acidophilus and S. mutans were procured from ATCC (Rockville, MD). Bacterial strains were grown in their appropriate broth medium at $37^{\circ} \mathrm{C}$ for 24 hours until a population between $1.0 \times 10^{8} \mathrm{cfu} \mathrm{ml}^{-1}$ and $5.0 \times 10^{8} \mathrm{cfu} \mathrm{ml}^{-1}$ was obtained. The inoculum was centrifuged at $13,400 \times g$ for two minutes. The supernatant was discarded and a highly concentrated bacteria cell pellet was obtained. The cell pellet was resuspended in $0.1 \%$ peptone water to a desired concentration based on optical density. Sintered HA discs, 9.5mm diameter and 1.8

$\mathrm{mm}$ thick were procured from Himed Inc. (Old Bethpage, NY, USA). HA discs were autoclaved at $121^{\circ} \mathrm{C}$ for 15 minutes, rinsed in ethanol, and stored in an oven at $70^{\circ} \mathrm{C}$ for 24 hours before use. Five microliters of the inoculum was pipetted and spread onto the HA disc surface then air dried in a biosafety hood for five minutes. Samples were treated at twenty second intervals, but the plasma brush only covered around 1/6 of the entire surface at any 
given time, hence equivalent treatment times are therefore reported as $1 / 6$ the total sample treatment time.

\subsubsection{Plasma treatment and brush construction}

A low temperature atmospheric plasma had previously been developed in our plasma research center [10]. The design was further refined to increase the size and stability of the plume while maintaining safe temperatures. The first of our designs of the atmospheric plasma brush was similar to a DBD, except we attempted to use a DC power supply. A glass tube was used as a dielectric barrier, a grounded copper ring electrode surrounded the tube, and a needle electrode was placed in the center of the tube. No discharge occurred, so we moved the ring electrode inside the glass tube. The discharge suffered from filament formation between the needle electrode and one location of the ring electrode. The needle electrode could not be placed in the exact center of the tube, therefore a discharge would occur toward the nearest location on the ring electrode. Once the plasma was formed, the conductivity of the plasma filament was greater than compared with other locations on the ring electrode, therefore the discharge would not spread to other locations on the ring. A typical DBD counters this problem by never forming a connecting filament to the ground electrode; plasma is confined near the needle electrode by the oscillating frequency. In fact, a second electrode is not necessary for sufficiently high frequency power supplies as the cathode acts as a sufficient ground electrode during half of the oscillation. The DC powered brush formed a large blade shaped plume at low temperature, so we saw promise in developing it further. At atmospheric pressure, nonequilibrium is reached through short pulses or in the case of large gradients of temperature, concentration, and mass flow velocity [24]. Our atmospheric plasma brush maintains a low temperature at the point of contact with 
the substrate, but the temperature is very high near the electrodes, especially the cathode. The flow rate and concentration of ionizing gas, in our case argon, is kept high near the electrodes through the use of a converging nozzle. The needle type electrodes confine the electric field near the electrode tips effectively creating a strong electric field local to the tip of the nozzle. This makes the electron and ion density very high allowing electrical discharge without arcing.

We next tried to increase the stability of the brush; since the filament was formed from the needle to one location on the ring electrode, the ring electrode was changed to a needle. Using a nylon prototyping machine, we created nozzles with varying shapes that confined argon flow near to the electrodes. The nylon nozzles were easily melted, so we tried ceramic tips commercially available for use in high temperature welding applications. The ceramic nozzles were inexpensive and mass produced with good tolerances. An acrylic prototyping material was made available to us, but still suffered from melting near the electrodes. A hand-held brush was produced consisting of an acrylic shaft with a threaded end, as shown in Figure 6.1. This allowed the ceramic tips to be threaded snuggly onto the shaft and allowed ease of use for hand-held operation in the mouth. A drawback was for the ceramic nozzle was that the plume was not as large as that observed in nozzles with oval shapes and suffered from instabilities. 


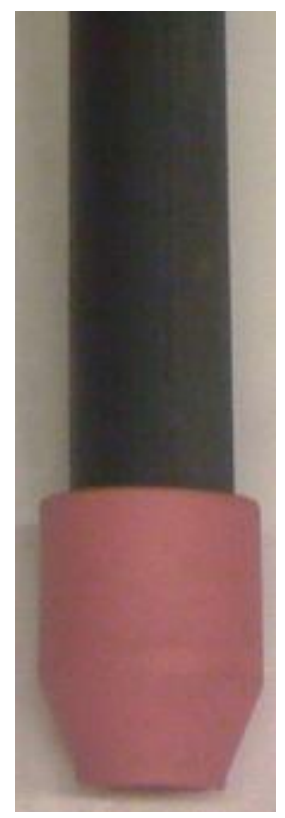

Figure 6.1. Photograph of a prototyped plasma brush with ceramic nozzle.

Through trial and error, it was found that the argon flow velocity around the electrodes needed to be higher to form a larger plume. In an attempt to create the higher velocity, the flow was constricted with inserts such as that seen below in Figure 6.2. We also were interested in the use of reactive gases added to the plasma in a separate line from the argon. Adding gas through a separate line would allow us to directly inject reactive gas into the plasma plume near the ground electrode. This may cause dissociation of the reactive gas without ionization. Trying to add this line proved difficult using the prototyping machine; extra material is needed during prototyping to act as a scaffold for subsequent layers. The extra material is difficult to clean out of small crevices such as that developed in the small reactive gas line also seen in Figure 6.2. Another problem was that the ground and cathode electrode must be placed such that the electrode tips are nearest one another otherwise a “Jacob's ladder" will form instead of a plasma plume. 


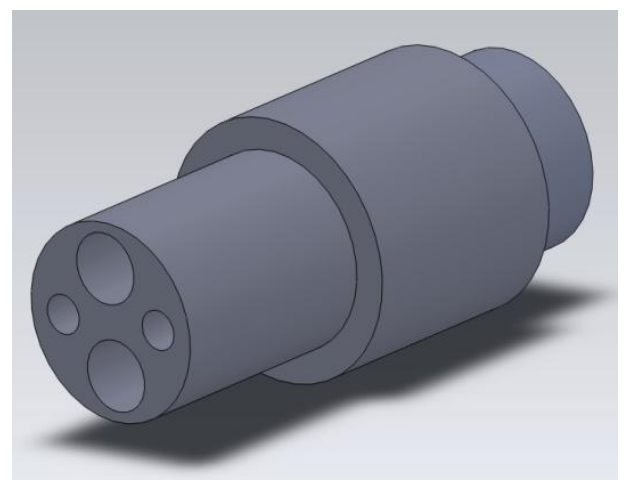

Figure 6.2. A depiction of a nozzle insert.

These challenges proved to be too difficult to surmount, so we decided to develop our own nozzles. We first tried a nozzle with a square tip that allowed a square glass insert and an oval tip that allowed a circular glass insert, as shown in Figure 6.3. The insert kept the electrodes from melting the nozzle; also, fixtures were made in the shaft and nozzle to keep the electrodes in the correct locations.
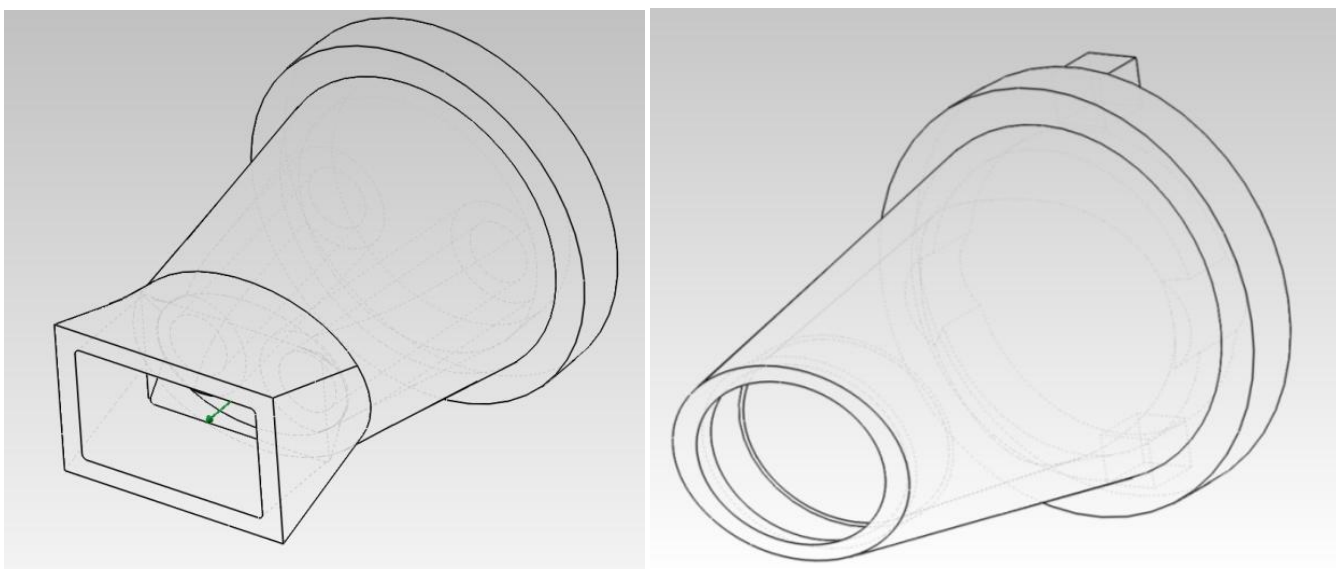

Figure 6.3. Depictions of nozzles created for the plasma brush; (left) a square glass insertable nozzle and (right) circular glass insertable nozzle.

The square nozzle still did not confine the argon around the electrode tips as well as previous oval nozzles, so the oval nozzle was further developed with the understanding that a 
final nozzle would be produced in ceramic. The final nozzle is inserted onto the shaft and held in place with a collar nut that threads onto the shaft as shown in Figure 6.4.
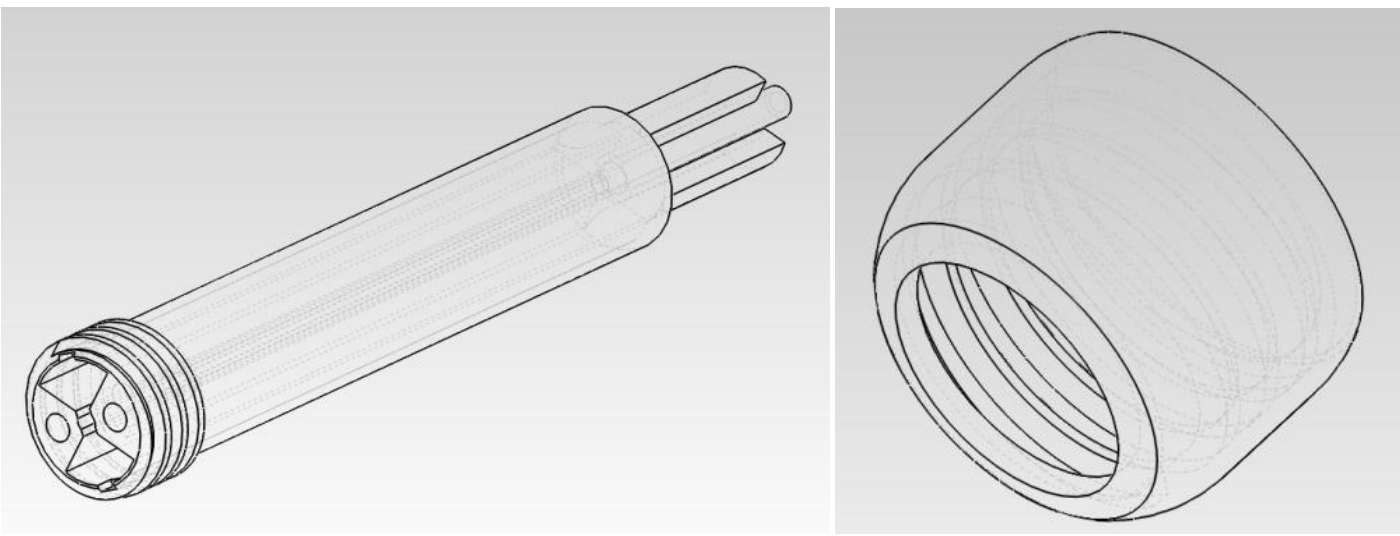

Figure 6.4. Depictions of the prototyped brush shaft (left) and collar nut for holding the nozzle in place (right).

Fixtures holding the electrodes in place were determined to obstruct the interior of the nozzle too much. Turbulence around the electrodes caused plasma instability. It was determined that fixtures in the shaft alone were sufficient to hold the electrodes in the correct locations. A final nozzle design, shown in Figure 6.5, was settle upon when a large and stable plume was produced. A $45^{\circ}$ nozzle was produced for better ergonomic use, also shown in Figure 6.5. 

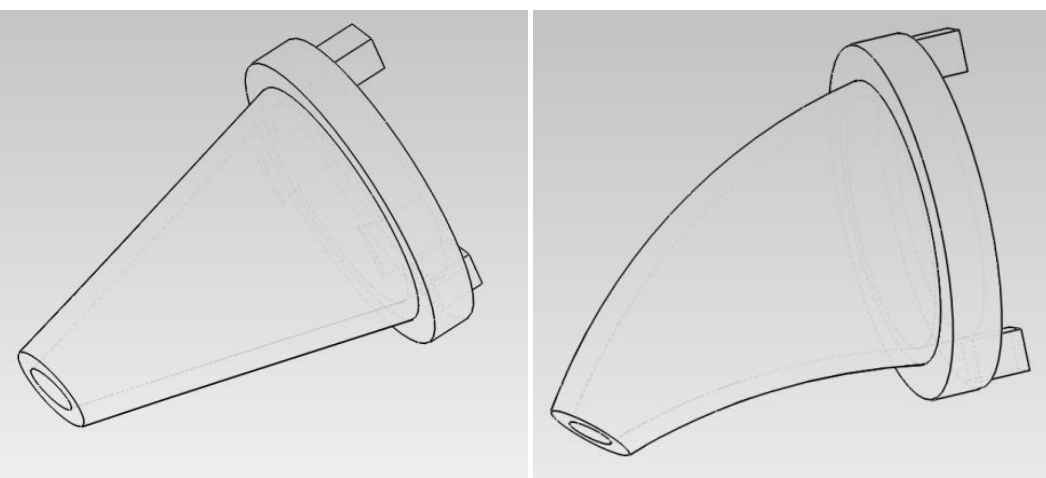

Figure 6.5. Depictions of the plasma brush nozzles used in the sterilization experiments; (left) the straight nozzle and (right) the $45^{\circ}$ nozzle.

Smaller nozzles were also created for rat trials as shown in Figure 6.6. For these, dentists requested a smaller form factor for the nozzle for easier use in the mouth.
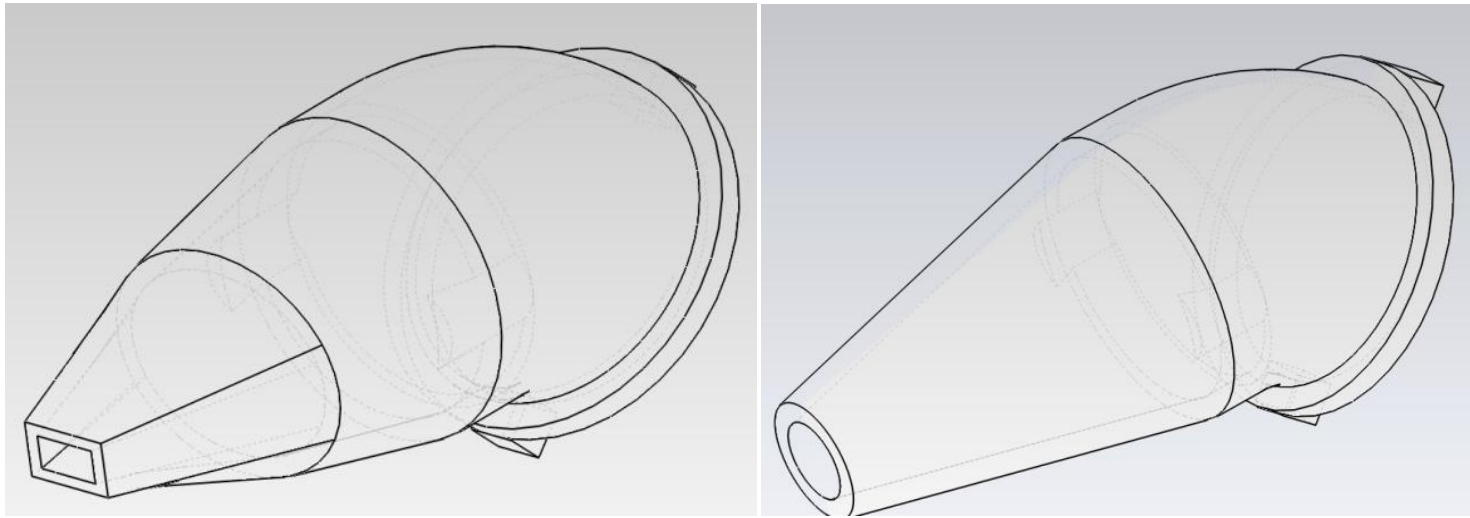

Figure 6.6. Depictions of nozzles designed for rat trials.

Argon, 99.9\% minimum purity, procured from Airgas, Inc. (Holt Summit, MO, USA), is flowed through the nozzle at $3000 \mathrm{sccm}$. An electric field is applied across the electrodes by a DC power supply (Pd 1556c, Power Design Inc., New York, NY, USA) and plasma is created. Plasma treatment is performed approximately $5 \mathrm{~mm}$ from the nozzle tip to sample surface. The power was kept around 3.0 W, i.e., $6.0 \mathrm{~mA}$ and approximately $0.50 \mathrm{kV}$. 


\subsubsection{Plasma Characterization}

The temperature of the plasma was measured with a Neoptix NMD-A portable fiber optic thermometer (Quebec City, Quebec, Canada). The probe used was a Neoptix T1 fiber optic temperature sensor with Teflon sheathing and a probe diameter of $1.15 \mathrm{~mm}$. Ozone was measured using an Eco Sensors A-21ZX ozone sensor (Newark, CA, USA). High resolution optical emission spectra was obtained using a Princeton Instruments (Acton, MA, USA) 750 mm focal length Acton SP-2756 Imaging Spectrograph. Images of the plasma were captured through a $780 \mathrm{~nm}$ MaxLine ${ }^{\circledR}$ laser clean-up filter. Images taken through the bandpass filter are two images averaged with ImageJ software.

\subsubsection{Bacterial enumeration}

After treatment, HA discs were placed in test tubes with $10 \mathrm{~mL}$ peptone water and vortexed for 30 seconds using a vortex mixer to remove the bacterium from the support medium surface. The solution was serially diluted with $0.1 \%$ peptone water and plated in MRS agar (Difco Labs., BD Dignostic Systems, Sparks, MD, USA) for L. acidophilus and tryptic soy agar (Difco Labs., BD Dignostic Systems, Sparks, MD, USA) for S. mutans. The plates were incubated plates are incubated for 48 hours and colonies are counted with a magnifying glass.

\subsubsection{SEM Characterization}

The effects of plasma treatment on the cell morphology and structure changes of the bacteria were examined using a scanning electron microscopy (SEM) (QUANTA 600F, FEI, Holland). The bacteria were placed on HA and treated the same as described in the previous 
section. Bacteria were affixed with a cacodylate and glutaraldehyde primary fixative solution, then rinsed in a secondary fixative of osmium tetroxide and cacodylate solution, followed by buffer rinses, milliwater rinses, and dehydration in EtOH. Samples are critical point dried and then coated with a thin layer of plasma sputtered platinum.

\subsubsection{Optical Emission Spectroscopy}

High resolution optical emission spectra were obtained using a Princeton Instruments $750 \mathrm{~mm}$ focal length Acton SP-2756 Imaging Spectrograph. A grating with $150 \mathrm{~g} / \mathrm{mm}$ blazed at 500nm was used. Spectra were collected through a $1 \mathrm{~m}$ UV-Vis fiber optic bundle.

\subsubsection{Computational Fluid Dynamics}

A computational fluid dynamics simulation was performed using Ansys Fluent. The nozzle used in the current plasma brush was modeled in Solidworks and imported into Ansys Fluent. The parameters for the CFD simulation were: $3000 \mathrm{sccm}$ argon flow rate, i.e., $8.114 \mathrm{~kg} / \mathrm{m}^{3} \mathrm{x}$ sec. A 3D model of plasma brush nozzle was inserted concentrically into a cylindrical control volume. The top of the control volume cylinder was two inches above the plasma brush nozzle outlet. At the top of the cylinder was a $100 \%$ volume fraction air boundary condition. The circumference of the cylinder is a pressure outlet allowing recirculation of fluid at room temperature and atmospheric pressure.

\subsection{Results and Discussion}

\subsubsection{Inactivation kinetics of oral bacteria}


Preliminary trials indicated a difference in killing rate depending on the initial bacterial seeding concentration (data not shown). Therefore, three different inoculum seeding concentrations were used in this study: a low inoculum concentration between $2.1 \times 10^{8}$ and $2.4 \times 10^{8} \mathrm{cfu} / \mathrm{mL}$, a medium inoculum concentration between $9.8 \times 10^{8}$ and $2.4 \times 10^{9} \mathrm{cfu} / \mathrm{mL}$, and a high inoculum concentration between $1.7 \times 10^{10}$ and $3.5 \times 10^{10} \mathrm{cfu} / \mathrm{mL}$. Five microliters of the bacterial cell suspension was spread over the $0.708 \mathrm{~cm}^{2}$ area of a HA disc. Change of bacterial surviving curves of $L$. acidophilus and S. mutans with plasma exposure time are shown in 6.7 and Figure 6.8, respectively. In the figures, " 0 " second represents the untreated controls that show the areal seeding concentration measured a posteriori. In general, it was found that the plasma deactivation rate did not vary among the two bacterial species studied. HA discs seeded with a low concentration of bacteria were completely deactivated after six to ten seconds of plasma treatment, as shown in figures 6.7-6.8. A treatment time of 13 seconds was required for full deactivation of both bacterial strains tested for the medium seeding concentration. Full deactivation of the bacteria was not achieved for high seeding concentrations. During preliminary trials, full sterilization was not achieved even after 160 seconds plasma treatment when the high bacterial seeding concentration was used, as shown in Figure 6.9. There was around a 1.5 to $2 \log$ reduction in $\mathrm{cfu} / \mathrm{mm}^{2}$ after six seconds plasma treatment, after which deactivation started to plateau to a maximum of $\sim 2-2.5 \log$ reduction in cfu/mm $\mathrm{mm}^{2}$. The temperature of the plasma brush used in this study is lower than $45^{\circ} \mathrm{C}$. To rule out bacterial deactivation by heating, bacteria on HA disks at the low seeding concentration were treated with $45^{\circ} \mathrm{C}$ heated argon gas to flow through the plasma brush without initiating plasma discharge. Heated argon gas blown over the bacteria showed less 
than $1 \log$ bacterial reduction. Therefore it can be inferred that the majority of deactivation occurred by plasma exposure other than heat.

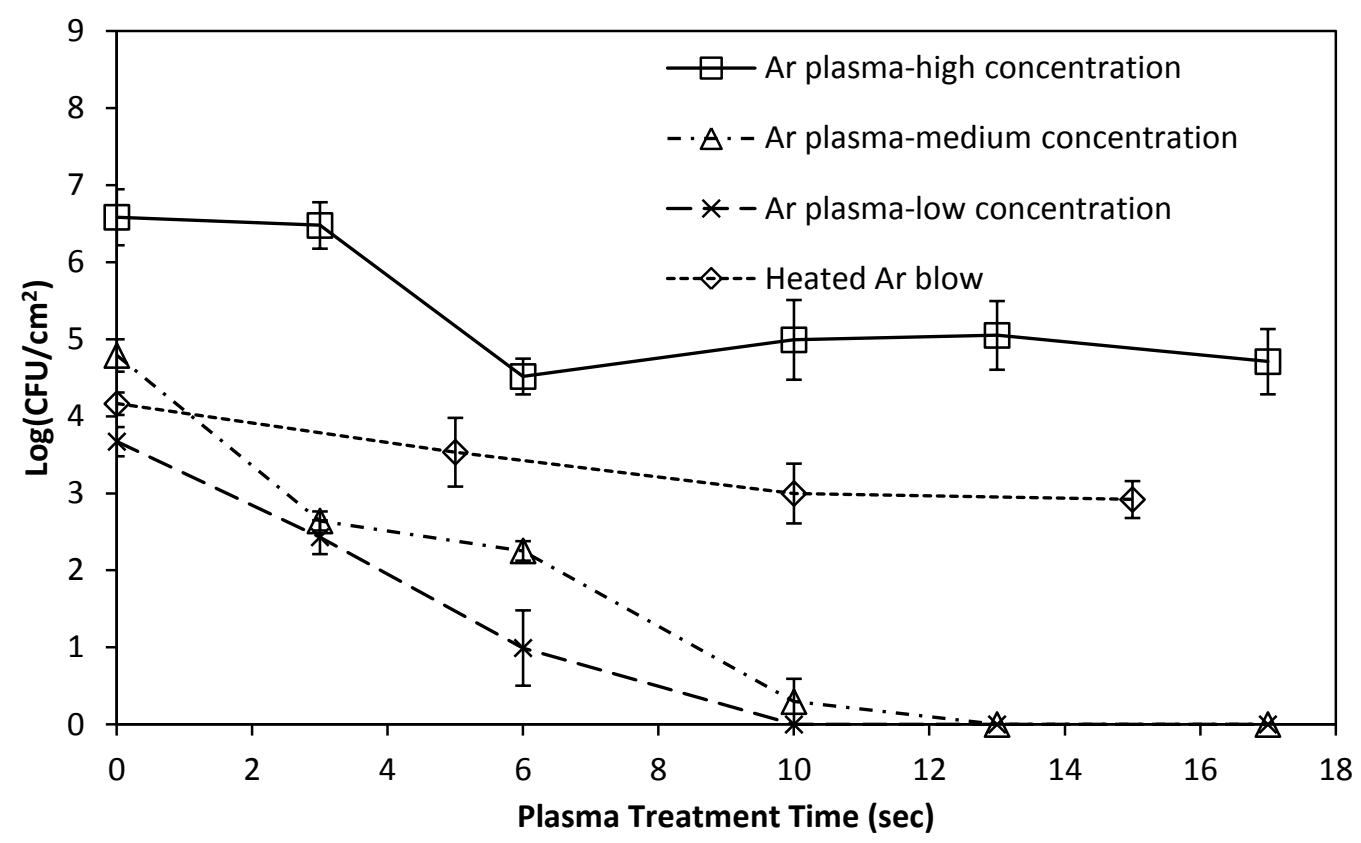

Figure 6.7. Survivability of L. acidophilus seeded on HA discs with different bacterial seeding concentrations and plasma exposure time. 


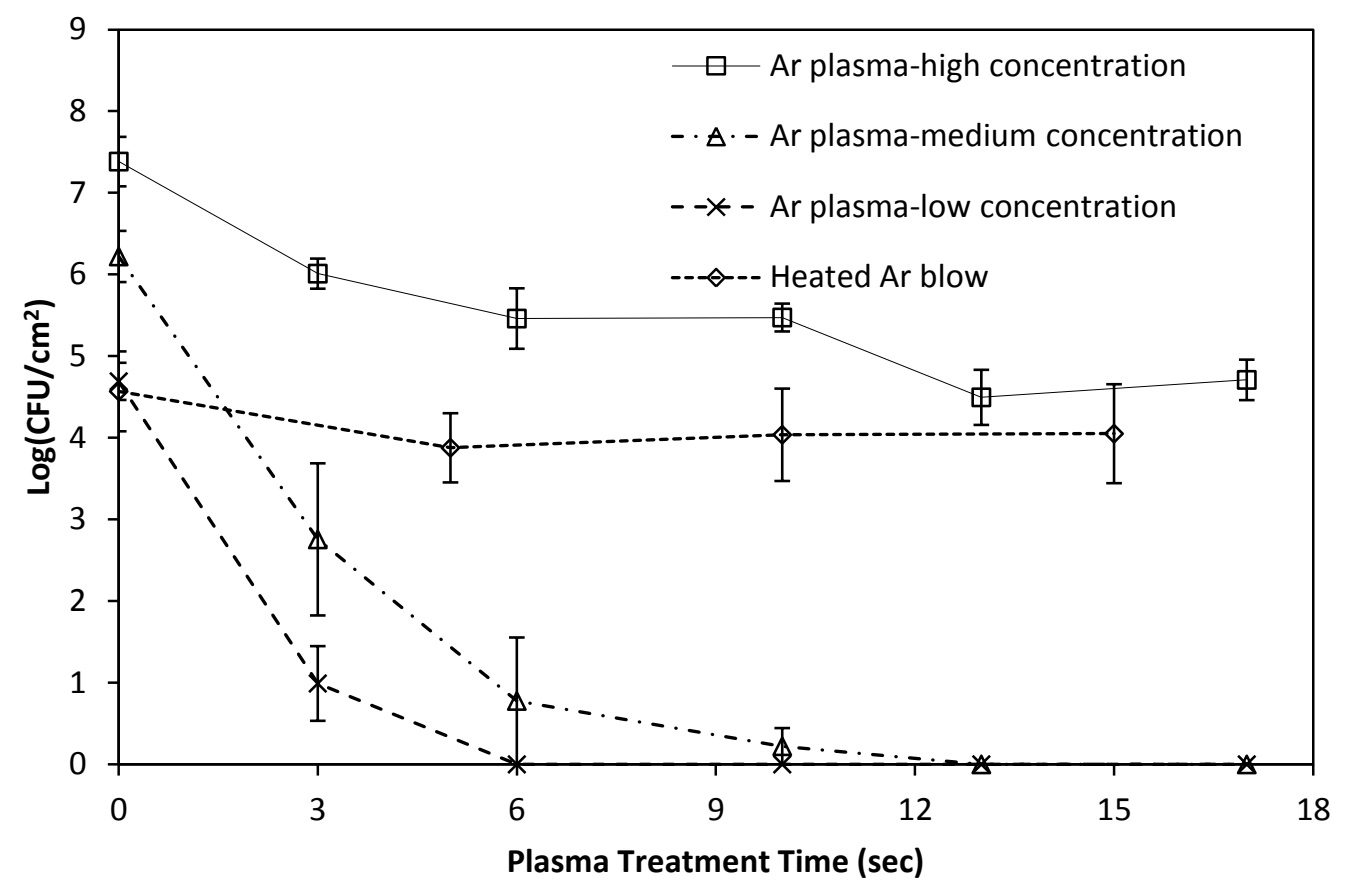

Figure 6.8. Survivability of S. mutans seeded on HA discs with different bacterial seeding concentrations and plasma exposure time.

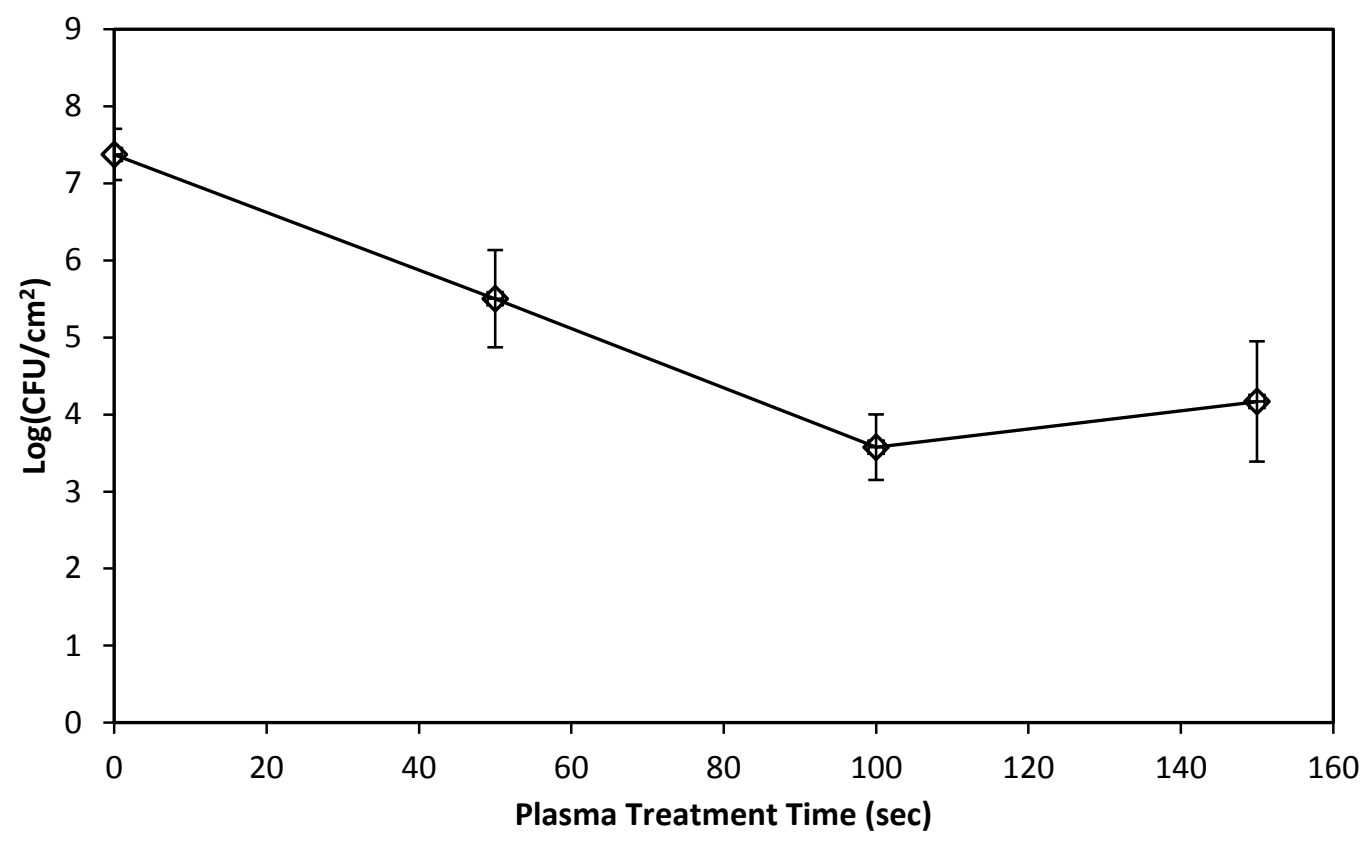

Figure 6.9. Survivability of $L$. acidophilus seeded on HA discs with high bacterial seeding concentrations at varying plasma exposure time. 
SEM was performed to analyze the bacterial morphology change before and after exposure to plasma. Figure 6.10 shows layering of L. acidophilus cells due to the high inoculum concentration. It can be seen that the top surface cells cell walls burst open and much intracellular contents were liberated, which confirmed the deactivation of the bacteria. It was also noted that the underlying cells remain intact after plasma exposure. This is consistent with the results shown in figures 6.7-6.8, i.e., not all of the bacteria were killed for the high inoculum concentration. Figure 6.11 shows the medium concentration of bacteria does not have as much layering due to its lower inoculum concentration. After three seconds of plasma treatment, surface roughening of the cell walls was observed. Cells burst open after six seconds of plasma treatment, and after 10 seconds cell walls were slightly more fragmented. SEM images of $S$. mutans with a high inoculum concentration are shown in Figure 6.12. Similar cell layering to that of L. acidophilus cells was also observed. The bacterial cells are nearly indiscernible after 10 seconds of plasma treatment time. There was less fragmentation of the cells compared with $L$. acidophilus. Individual cells can still be seen, but they appeared as melted clusters. SEM images of $S$. mutans with the medium seeding concentration are shown in Figure 6.13, in which cell walls were easily distinguished and intracellular contents can be seen spilling out. SEM images of the sample cross-sections for both $L$. acidophilus and S. mutans are shown in Figure 6.14. The cell layering due to a high seeding concentration was evident. It can been clearly seen that the cell debris and partially destroyed cells reside on the surface while three to four layers of intact cells remain underneath. The physical size of $L$. acidophilus cells is around $1.5 \mu \mathrm{m}$ in length and around $0.9 \mu \mathrm{m}$ in diameter. It was expected that around three to four layers of L. acidophilus cells were formed when they were seeded with the high concentration inoculum. It is difficult to 
estimate the number of cell layers destroyed based on the cross-section images alone. The cell debris layer is very thin when compared with the underlying cell layers. The SEM images shown in Figure 6.14 confirmed that only the top layer of cells was destroyed by plasma treatment. Yang et al. had proposed the layered bacteria concept based on the physical size of bacterial cells and described the incomplete plasma bacterial deactivation due to overlying cell debris blocking direct contact of plasma [6].

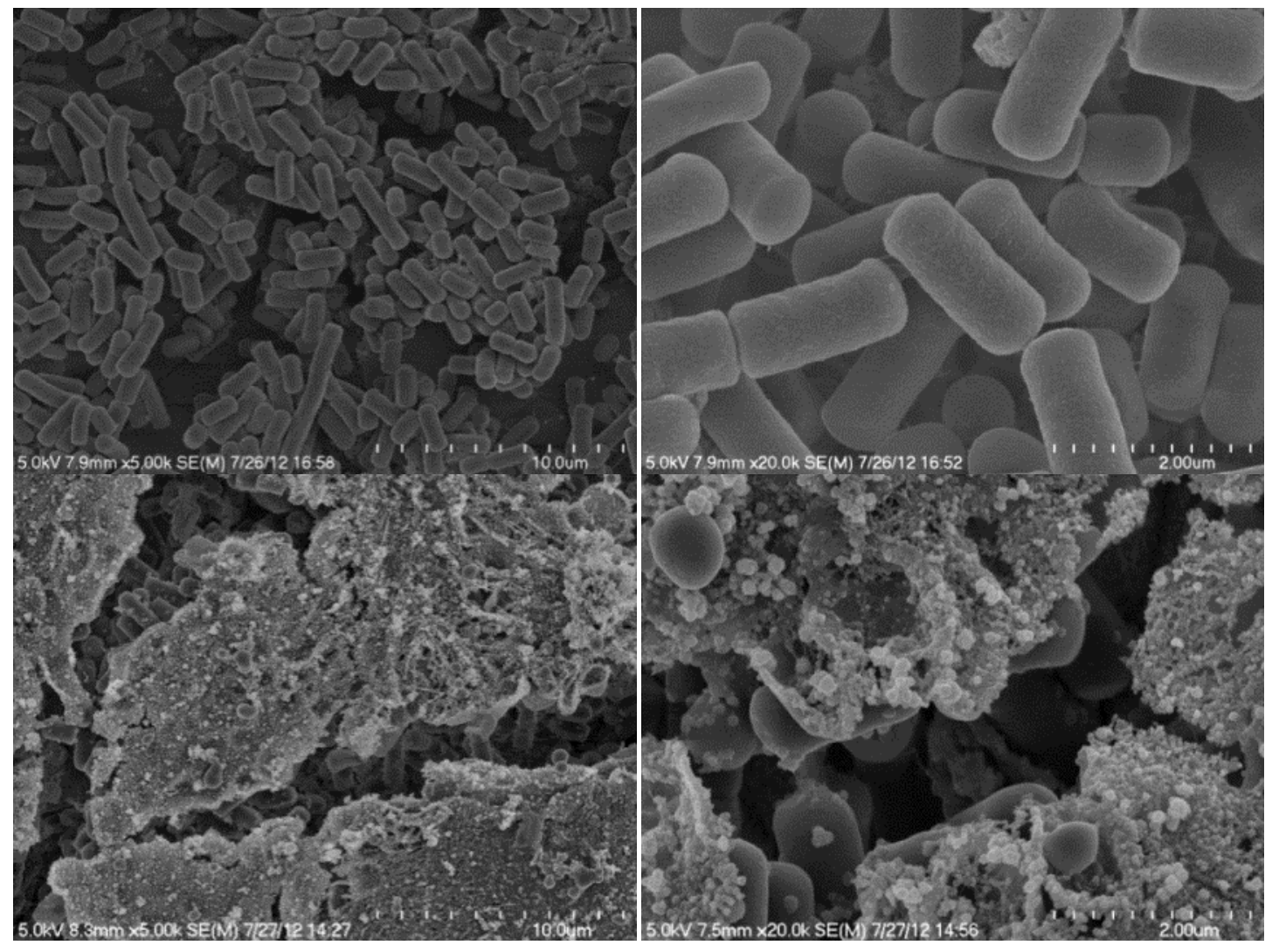

Figure 6.10. SEM images of the high seeding concentration of $L$. acidophilus cells on HA discs with no treatment (top) and after 10 seconds plasma treatment (bottom). 


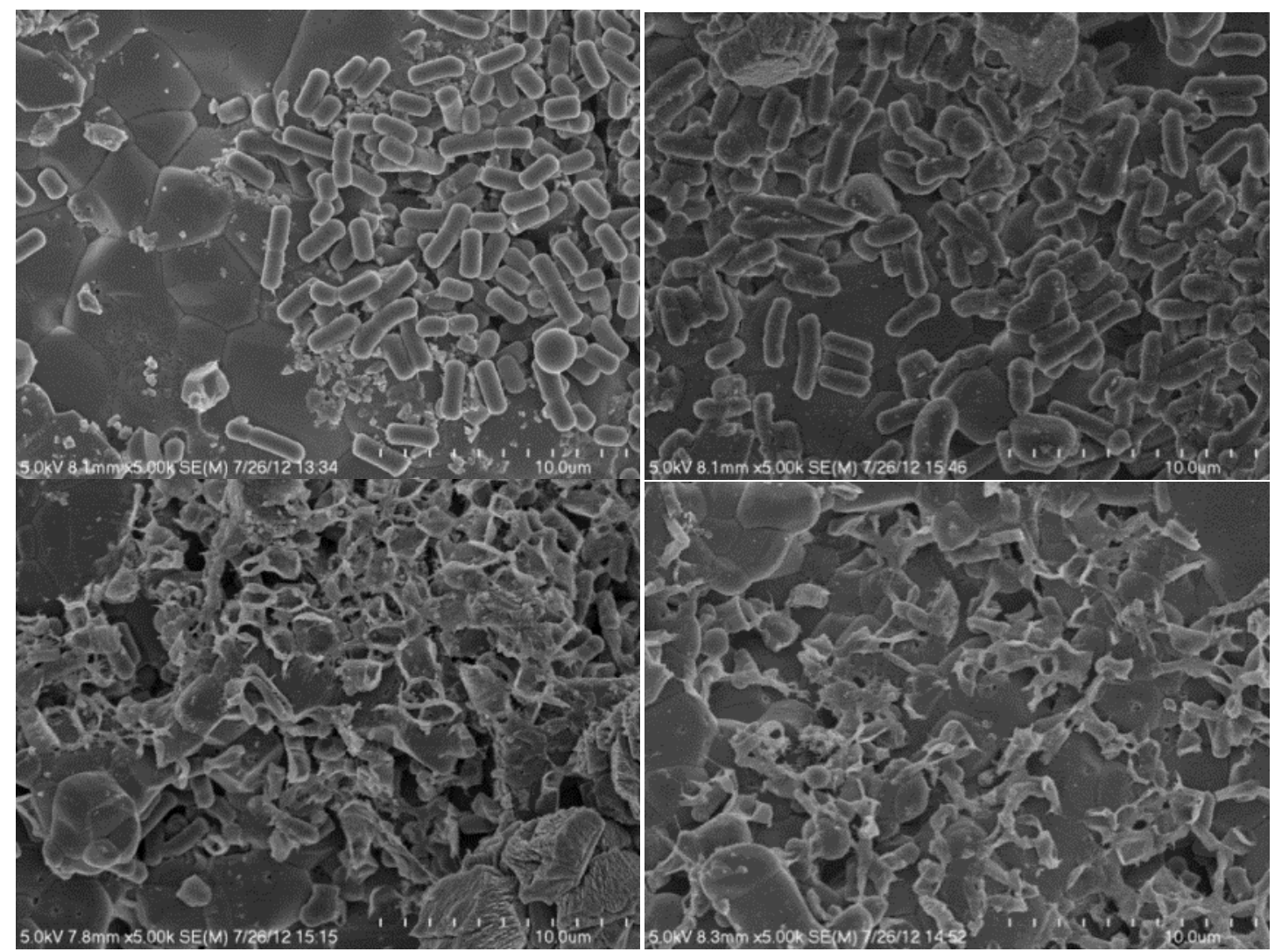

Figure 6.11. SEM images of the medium seeding concentration of L. acidophilus cells on HA discs without treatment (top-left), after 3 seconds plasma treatment (top-right), after six seconds plasma treatment (bottom-left), and after 10 seconds plasma treatment (bottomright).

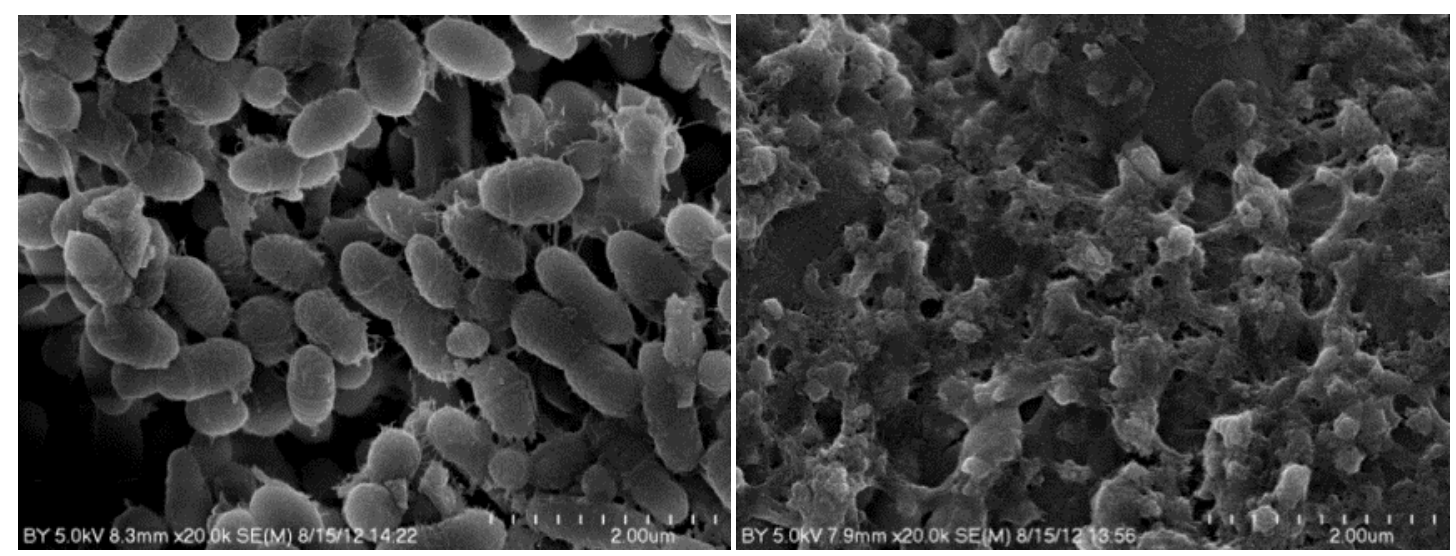

Figure 6.12. SEM images of the high seeding concentration of $S$. mutans cells on HA discs without treatment (left) and after 10 seconds plasma treatment (right). 

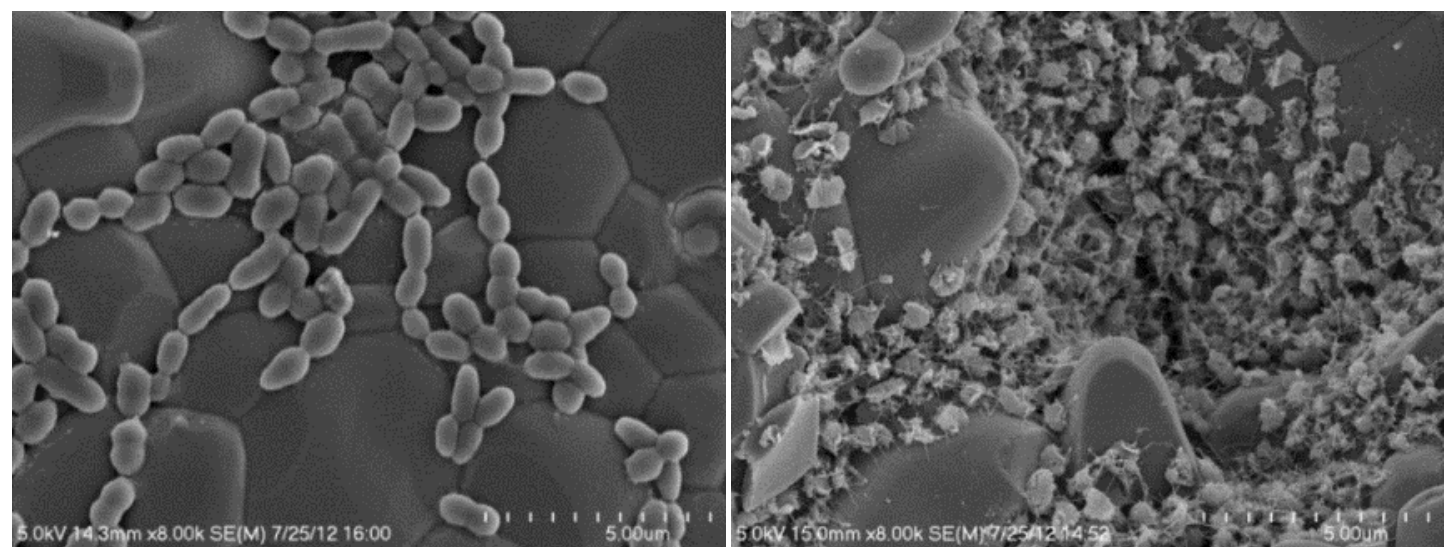

Figure 6.13. SEM images of the medium seeding concentration of $S$. mutans cells on HA discs without treatment (left) and after 10 seconds plasma treatment (right).

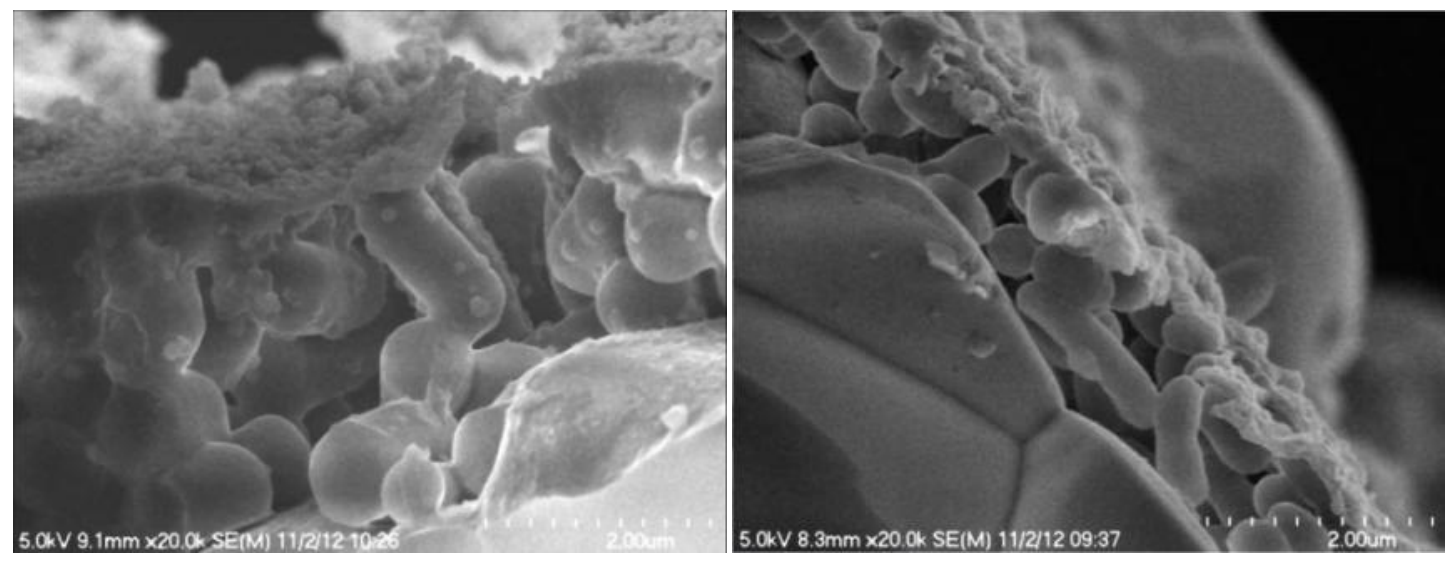

Figure 6.14. SEM images of the high seeding concentration of L. acidophilus cells (left) and $S$. mutans (right) on HA discs after 10 seconds plasma treatment.

\subsubsection{Oxygen Effects on Deactivation Rate}

The fast rate of killing obtained in this study was further substantiated with SEM images which showed extensive cellular damage. This damage is considerably intense compared to other studies in which bacteria were deactivated, but the bacterial cells remained intact $[18,24,25]$. There are many possible mechanisms for the bactericidal activity of the plasma, e.g., electron bombardment of cell membranes, charge accumulation, chemical reactions such as oxidation, destruction of nucleic acids by ultraviolet radiation, and ablation 
of the cell membrane [14,24]. In all cases, the site of action for the plasma deactivating mechanisms is either at the cell membrane or at DNA. Much research has been performed to elucidate the mechanisms of deactivation by atmospheric plasmas, but these are typically dielectric barrier discharge plasmas operating at high frequencies, i.e., radiofrequency, microwave frequency, pulsed DC, etc.[8,11,12,15,26-28] This high frequency may interrupt chemical reactions compared to our DC powered plasma brush [29]. In addition, much of the atmospheric plasma research is performed with helium as the base ionizing gas. Argon plasmas may achieve higher electron temperatures capable of dissociating higher energy bonds and therefore creating different chemical species [11]. Further, the concentration of various chemical species generated in this process will surely be different between argon and helium as the base ionizing gas; for instance, plasmas with argon are more efficient in creating reactive oxygen species compared to helium when oxygen is present. Since most authors suggest reactive oxygen species to be the major contributor to deactivation, we performed a number of experiments adding oxygen into the plasma to study the effect of oxygen on deactivation effectiveness. Instability of plasma was noted when oxygen gas was pre-mixed with argon. Instability of plasma is due to the highly electronegative oxygen which scavenges electrons that maintain the plasma. This is termed as dissociative attachment instability [30]. In contrast, a stable plasma can be maintained when oxygen was injected into the plasma near the ground electrode with a flow rate up to $1750 \mathrm{sccm}$. Therefore, oxygen effects were examined by injecting oxygen into the plasma near the grounded electrode. An increasing amount of ozone was measured with increasing amounts of oxygen injected as shown in Figure 6.15. Ozone is a very strong oxidizer. Since ozone production was maximized at 1500 sccm oxygen addition, we attempted deactivation at 1500 
$\mathrm{sccm} \mathrm{O}_{2}$ addition near the ground electrode, as shown in Figure 6.16. We chose $50 \mathrm{sccm}$ as a low $\mathrm{O}_{2}$ flow rate. Next, we performed deactivation in a sealed jar with a water bubbler type one way valve. The $0.5 \mathrm{~L}$ jar was purged for 1 minute with argon at $3 \mathrm{~L} / \mathrm{min}$ to lower the oxygen concentration in the jar.

Huang et al. found oxygen addition into the plasma can either increase or decrease the deactivation rate depending on supporting media [9]. Our results are similar to their results for deactivation on nutrient broth and standard methods agar, i.e., addition of oxygen caused lower or similar deactivation rates. Oxygen addition had little effect on deactivation rate for both L. acidophilus and S. mutans, as shown in Figure 6.17 and Figure 6.18 respectively. Also, deactivation in a reduced oxygen environment had little effect on the deactivation rate for both bacterial strains tested.

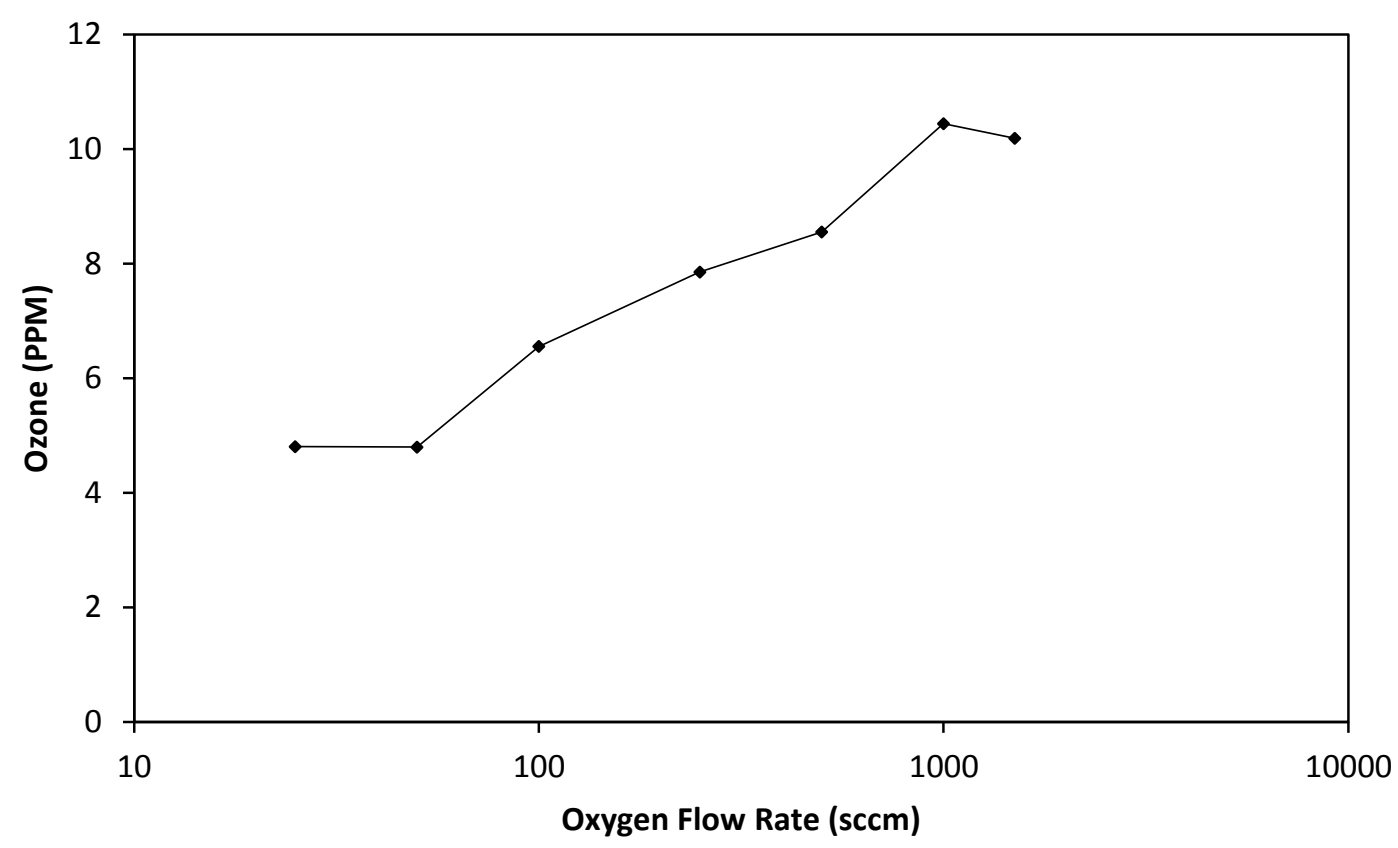

Figure 6.15. Ozone creation with different amounts of oxygen added near the ground electrode. 


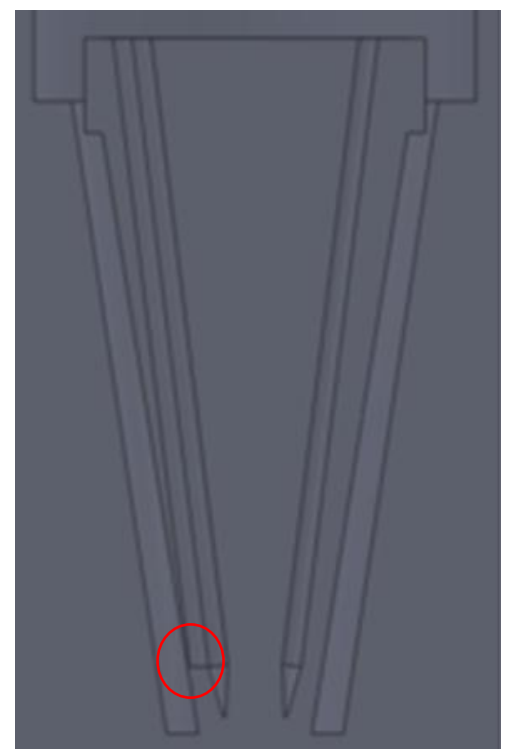

Figure 6.16. A depiction of the electrode and gas line set up with respect to the plasma brush nozzle.

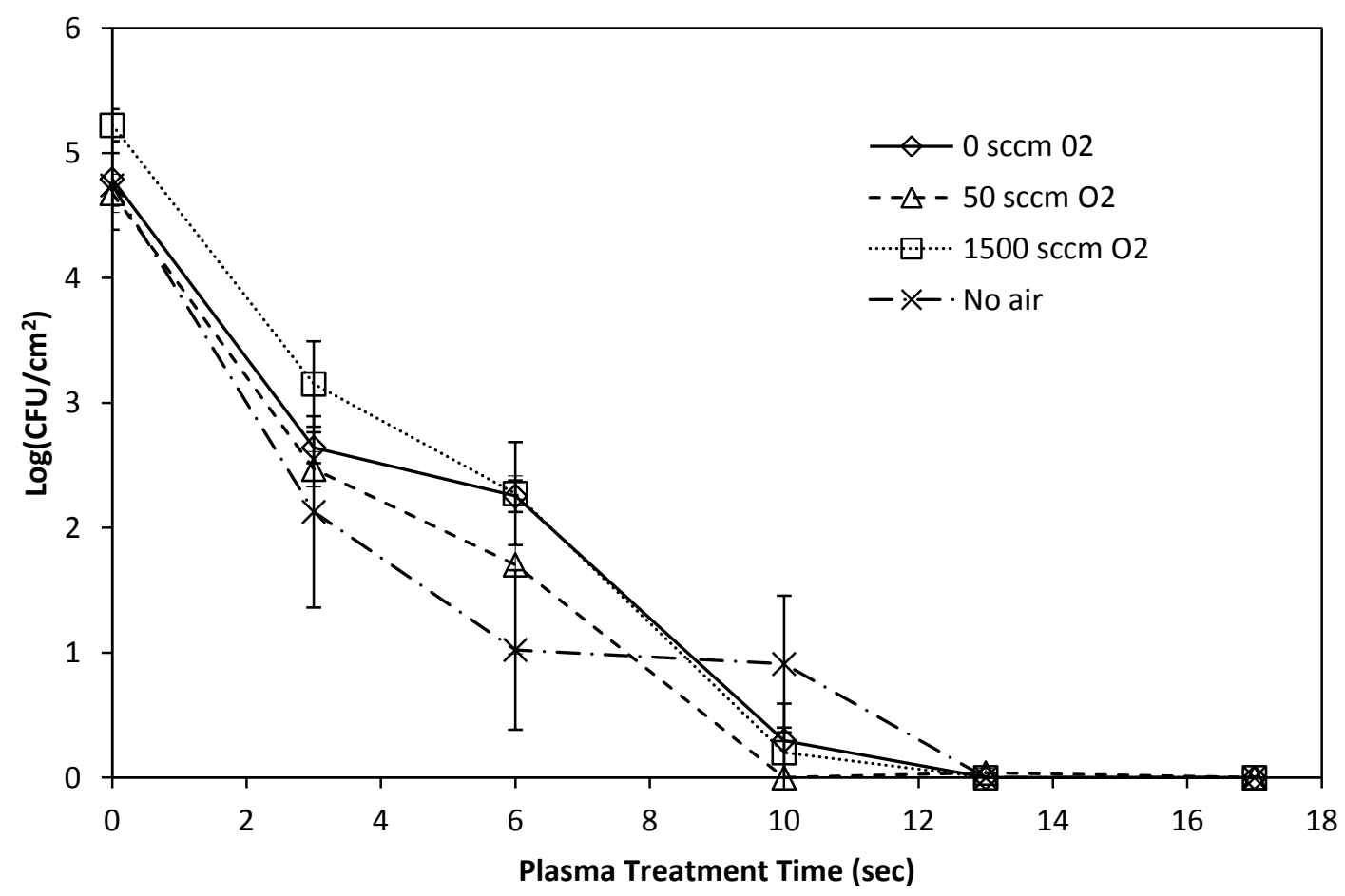

Figure 6.17. Survivability medium seeding concentration of L. acidophilus seeded on HA discs with different plasma exposure times and oxygen conditions. 


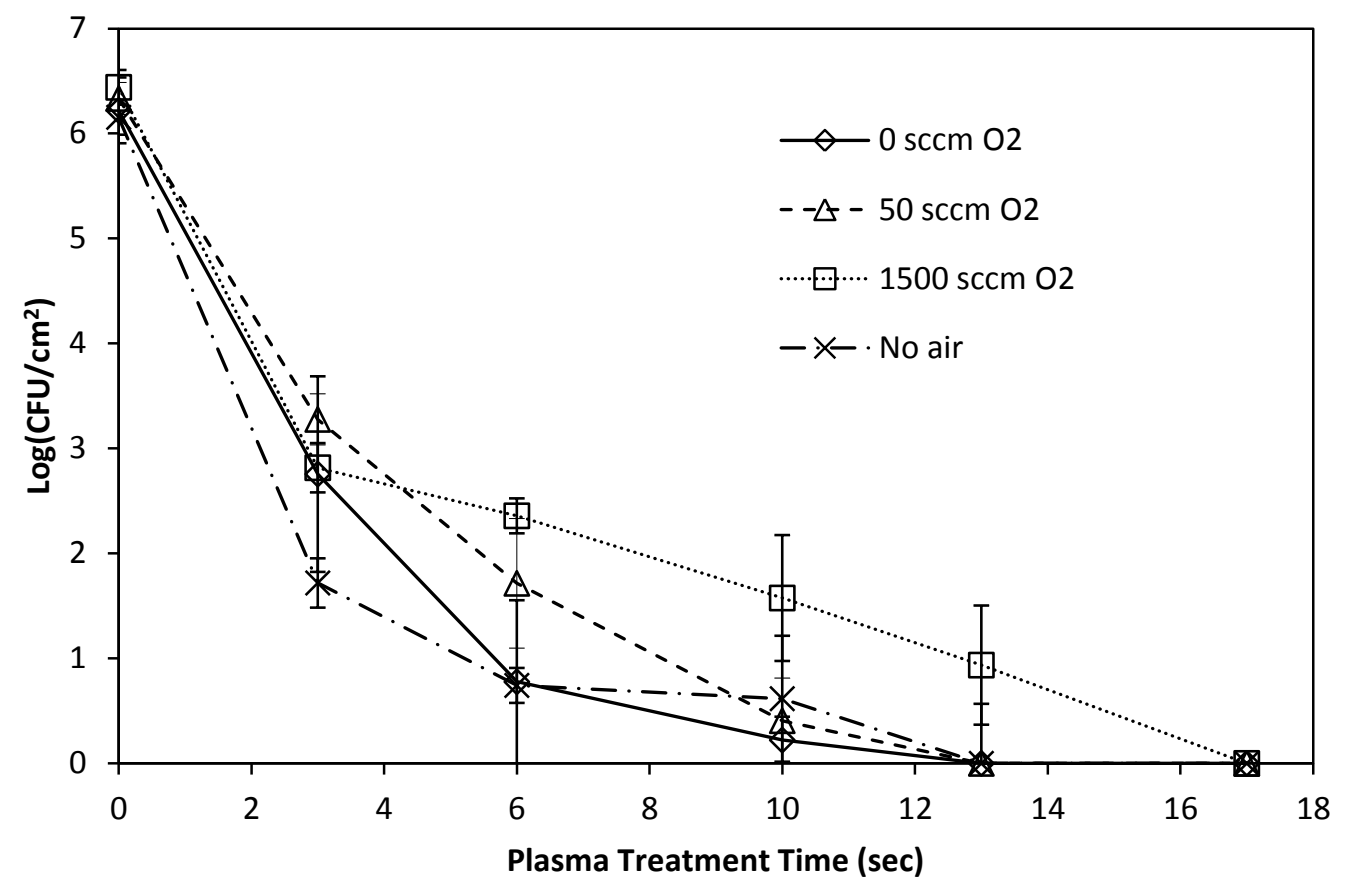

Figure 6.18. Survivability medium seeding concentration of S. mutans seeded on HA discs with different plasma exposure times and oxygen conditions.

\subsection{Plasma Brush Characterization}

\subsubsection{Optical Emission spectroscopy}

Optical emission spectroscopy was performed with the optical fiber in line with the plasma blade, as shown in Figure 6.19. To increase the number of reactive oxygen species, $50 \mathrm{sccm}$ of $\mathrm{O}_{2}$ was added by two methods: 1) mixing with argon prior to entering the plasma brush, and 2) through a small tube near the ground electrode. Optical spectra of the plasma formed in atmosphere results in very few reactive oxygen species emitting in the 200-900 nm range, as shown in Figure 6.20. The only oxygen peaks found were $\mathrm{OH}$ at $309 \mathrm{~nm}$ and monatomic oxygen at 777 and $844 \mathrm{~nm}$. Argon plasma with $\mathrm{O}_{2}$ injected into the plume near the ground electrode gave higher and lower values of monatomic oxygen depending on the 
location of the fiber optic probe. Since Perni et al. and Goree et al. both found monatomic oxygen to be a major contributor to bacterial deactivation, spectra from our plasma was observed through an optical bandpass filter with center wavelength of $780 \mathrm{~nm}$. In this method, the spatial distribution of the monatomic oxygen may be observed in order to remove discrepancy from fiber optic probe location. Figure 6.21 and Figure 6.22 shows the addition of oxygen into the plasma did not result in an increase in the observed monatomic oxygen peak, but instead decreased the observed emission for both types of oxygen injection. Therefore, monatomic oxygen may remain a major mechanism of deactivation, but oxygen addition did not cause an increase in monatomic oxygen. Interestingly, the voltage increases with increasing oxygen addition when added as a mixture, but the voltage stays the same when added through the needle. At around $2000 \mathrm{sccm}$ of added oxygen through the needle, the voltage even starts to drop. The injection of oxygen through mixing with argon and passing between electrodes versus the injection of oxygen into the plume near the ground electrode may result in very different chemical products. Plume temperature increases with increasing voltage. Figure 6.23 shows that adding oxygen as mixed results in much higher voltages which translated into unsafe temperatures. Further, as mixed resulted in an unstable plasma most likely unsuitable for clinical use. 


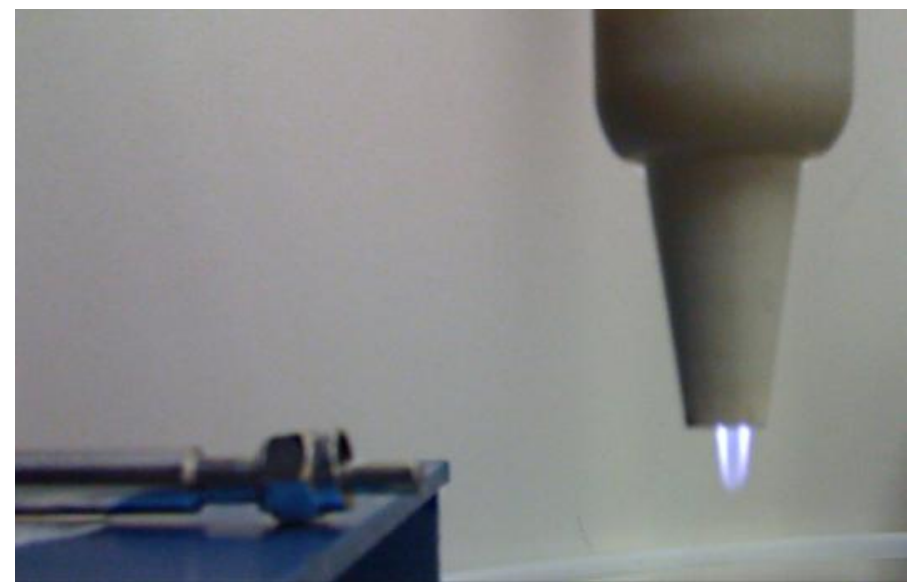

Figure 6.19. Optical fiber pointed toward the plasma brush running parallel with the blade.

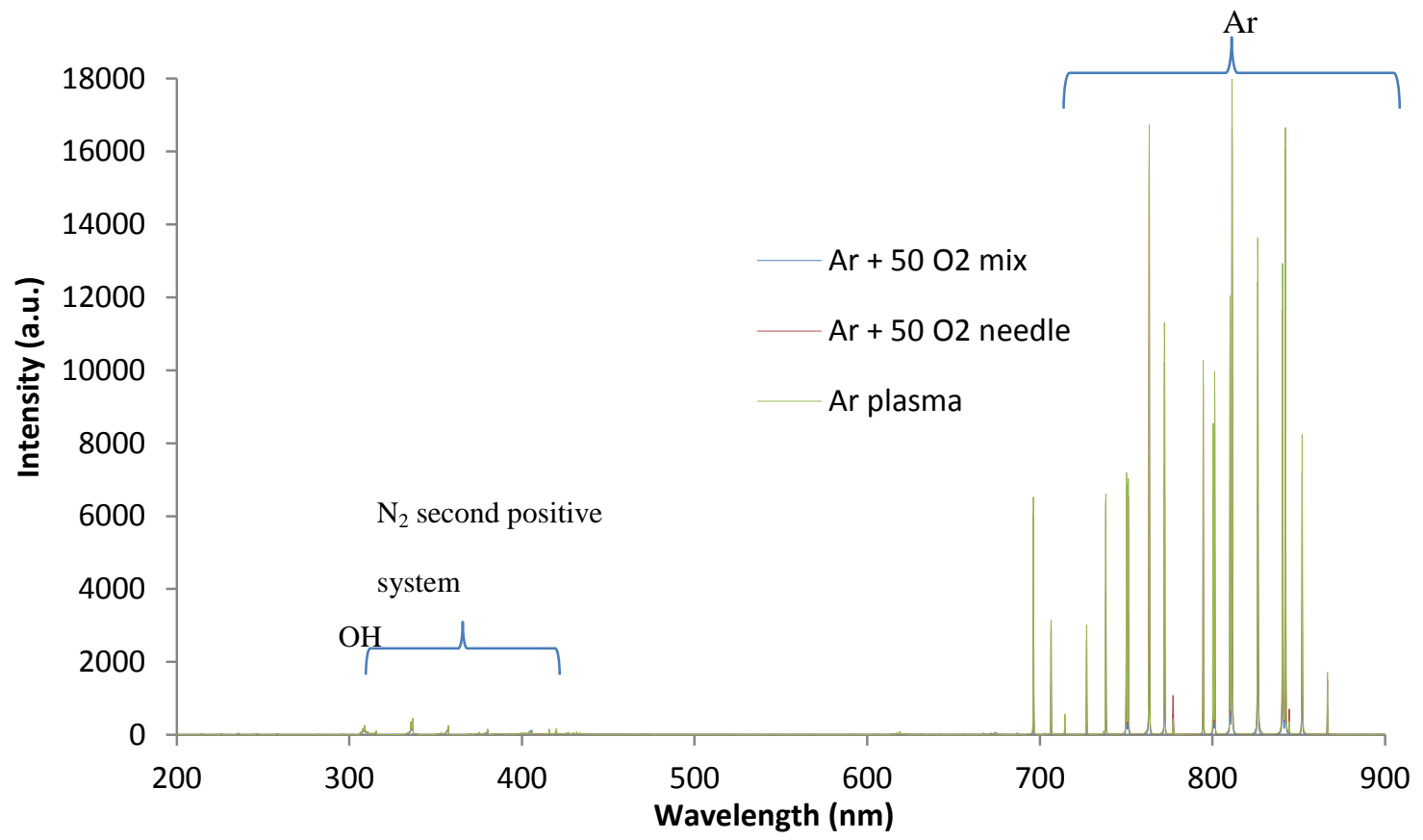

Figure 6.20. Optical emission spectra of argon plasma. 


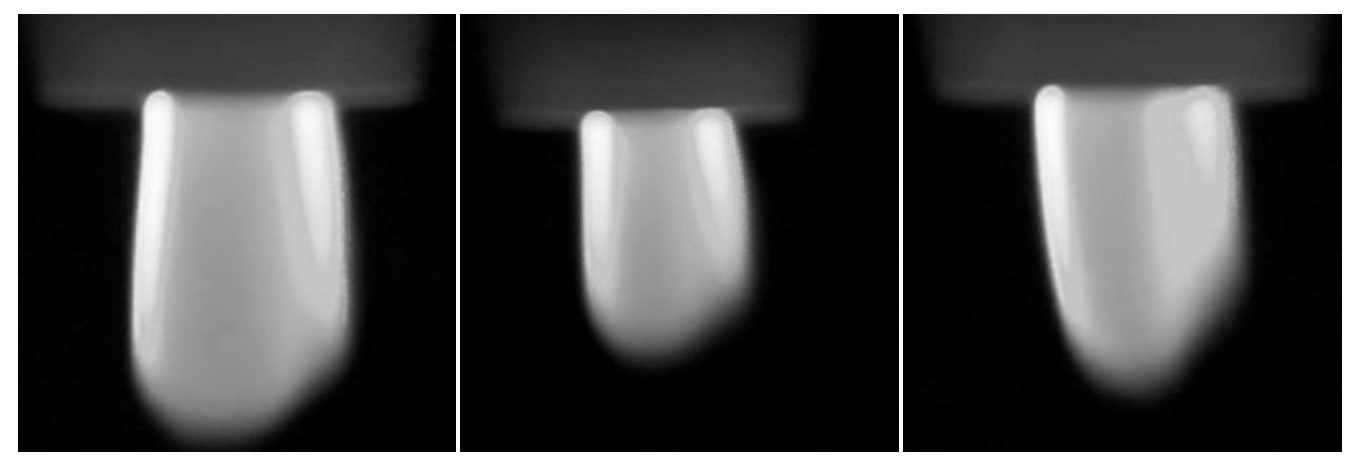

Figure 6.21. Light emitted from the plasma at the $777 \mathrm{~nm}$ monatomic oxygen peak with $\mathrm{O}_{2}$ addition near the ground electrode at $0 \mathrm{sccm}$ addition (left), $5 \mathrm{sccm}$ addition (middle), and 50 sccm addtion (right) as observed through a $780 \mathrm{~nm}$ center wavelength bandpass filter.

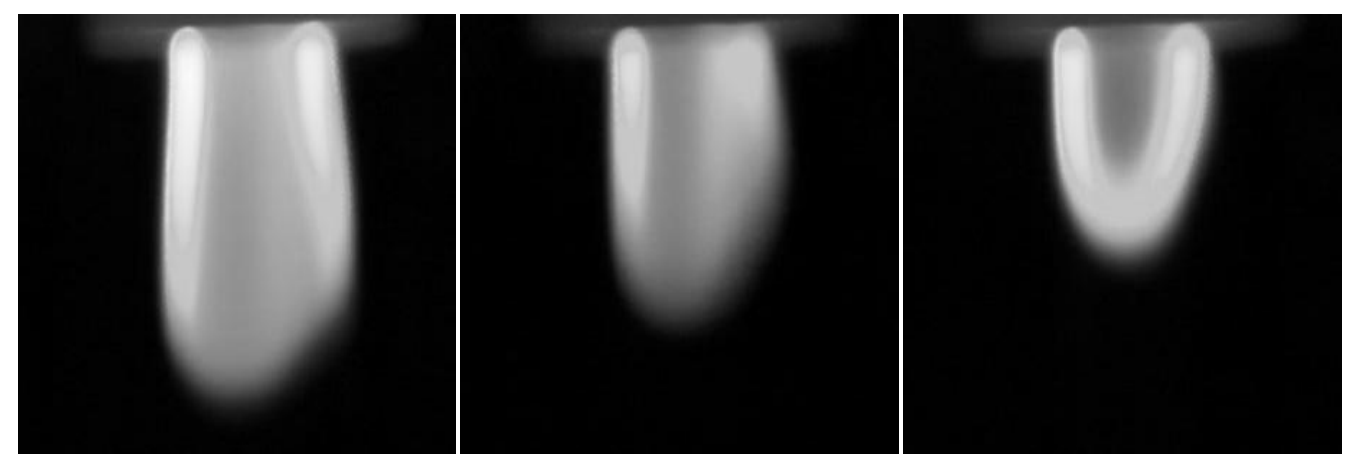

Figure 6.22. Light emitted from the plasma at the $777 \mathrm{~nm}$ monatomic oxygen peak with $\mathrm{O}_{2}$ addition as mixed at $0 \mathrm{sccm}$ addition (left), $5 \mathrm{sccm}$ addition (middle), and $50 \mathrm{sccm}$ addtion (right) as observed through a $780 \mathrm{~nm}$ center wavelength bandpass filter. 


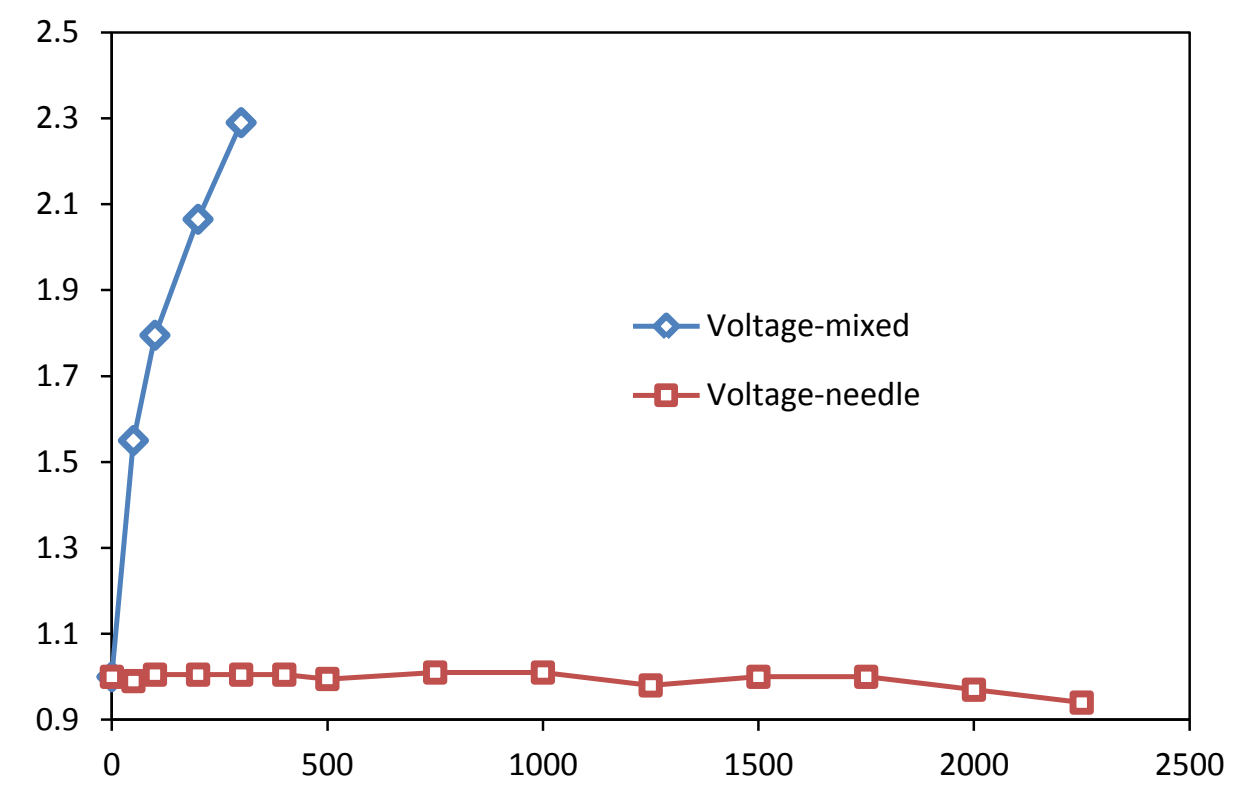

Figure 6.23. Light emitted from the plasma at the $777 \mathrm{~nm}$ monatomic oxygen peak with $\mathrm{O}_{2}$ addition as mixed at $0 \mathrm{sccm}$ addition (left), $5 \mathrm{sccm}$ addition (middle), and $50 \mathrm{sccm}$ addtion (right) as observed through a $780 \mathrm{~nm}$ center wavelength bandpass filter.

\subsubsection{Optical Emission Spectroscopy Performed During Plasma Sterilization}

Most researchers hypothesize oxidation of bacterial cell walls and intracellular material by reactive oxygen species to be the main contributor to sterilization. Vujosevic et al. found sterilization of $E$. coli by oxygen plasma resulted in observable $\mathrm{N}_{2}$ and $\mathrm{CO}$ peaks by OES at $337 \mathrm{~nm}$ and $519 \mathrm{~nm}$, respectively [31]. Although Vujosevic's experiment was performed in vacuum, we hypothesized that, if sterilization is truly caused by oxidation, these peaks should be observed with the use of our plasma brush as well. The optical fiber in this case was set up to collect spectra just above an HA disk, as shown in Figure 6.24. Bacteria was prepared the same as the high concentration sterilization experiments from previous sections. Sterilization was allowed to occur for 10 seconds before spectra was collected to ensure oxidation was given a chance to take place. Since $\mathrm{O}_{2}$ addition near the ground electrode added to the monatomic oxygen peaks, it was again used for this experiment to see 
if increased oxidation could be observed. Spectra, shown in Figure 6.25 and Figure 6.26, NO and $\mathrm{CO}$ peaks were not higher than that found with plasma on bare HA. Any peak present is considered to be existing due to species created naturally in atmosphere. Peaks are slightly lower when oxygen is added. This is due to the quenching effect that oxygen has on the plasma.

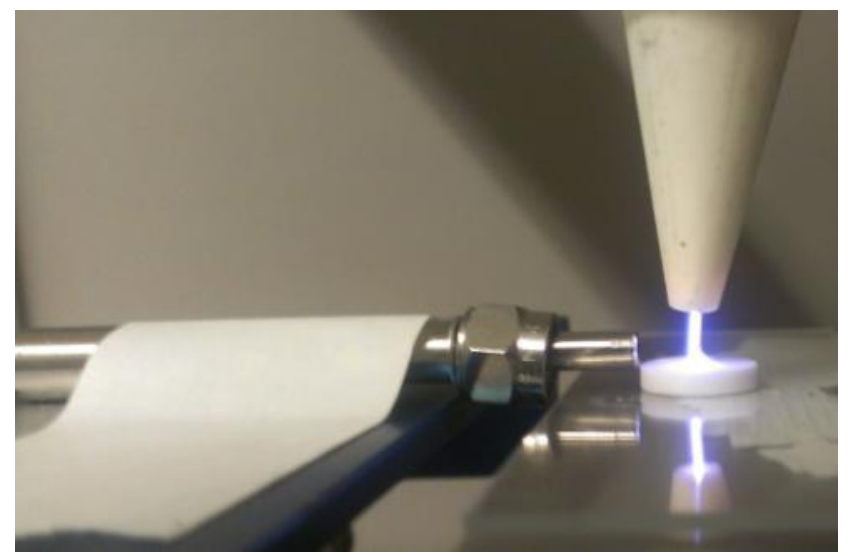

Figure 6.24. Optical emission spectroscopy of plasma during sterilization.

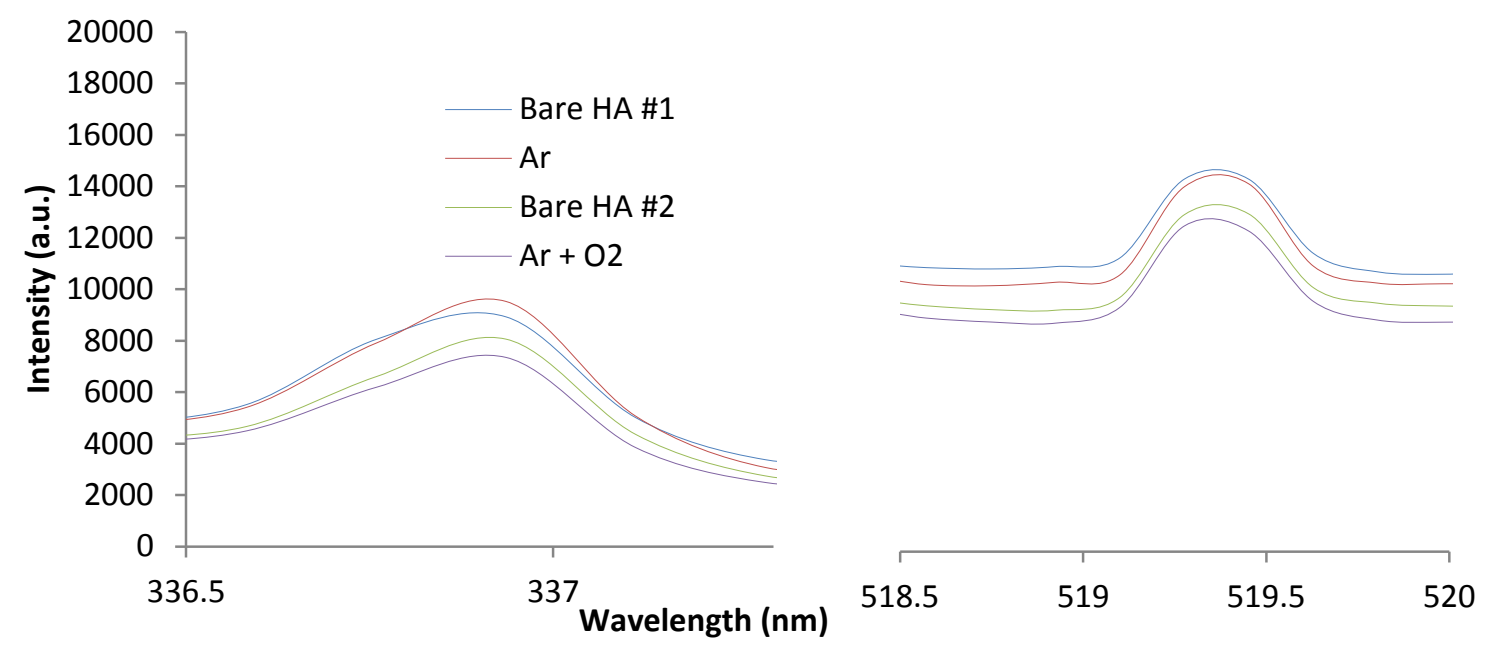

Figure 6.25. OES spectra of plasma during sterilization of S. mutans. 


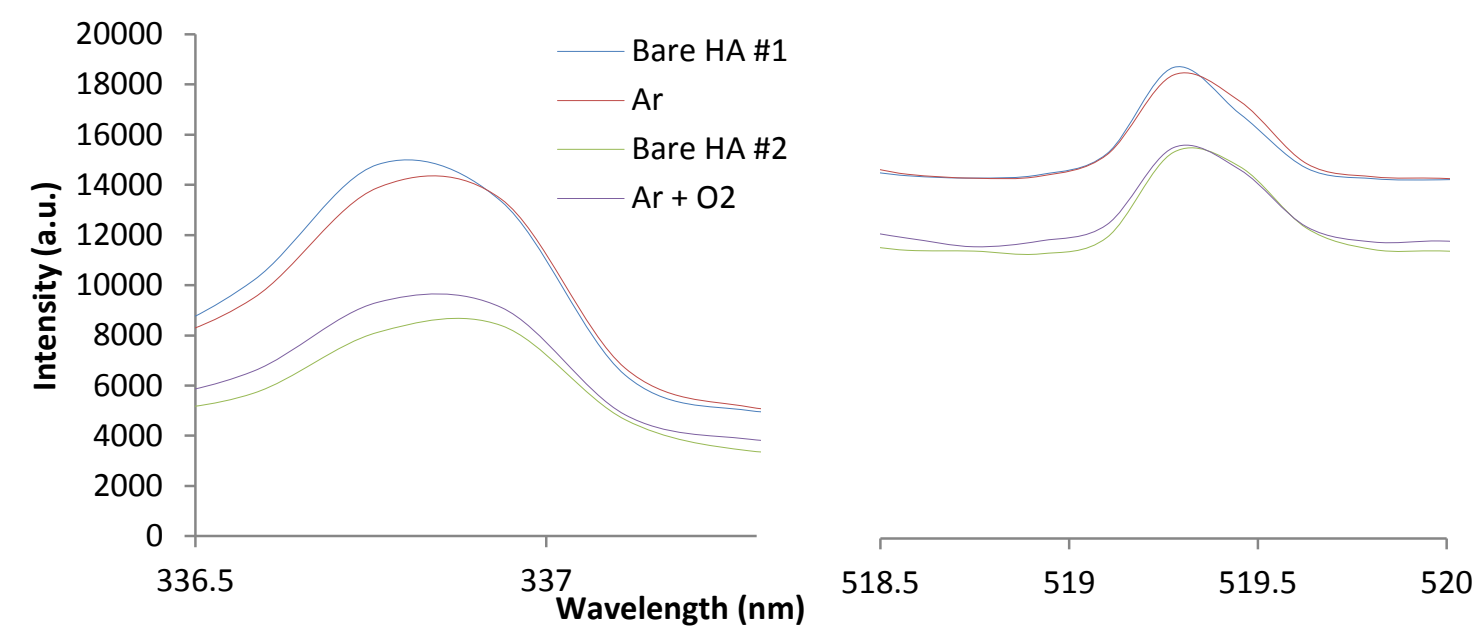

Figure 6.26. OES spectra of plasma during sterilization of L. acidophilus.

\subsubsection{Computational Fluid Dynamics}

Atmospheric plasmas are difficult to model, they are typically non-maxwellian and consist of a large number of particles of numerous types of chemical species and charge [32]. Various reactions occur depending on the collision cross-sections of each molecule and heat production is caused collision. This high complexity makes simulation of plasma very difficult. Therefore, the CFD simulation performed here does not take into account any magnetic/electric field or charged particle interactions, e.g. no chemical reactions are simulated; only the fluid flow is simulated. CFD of our plasma brush emitting argon into atmosphere results in a low concentration of atmosphere near the surface of the HA disk. Argon concentrations are almost always above $\sim 60 \%$ near the disk surface, as shown in Figure 6.27 (left). A picture of the plasma brush next to the simulation shows plasma is most likely confined to areas of high argon concentration. The simulation shows a boundary layer is formed acting as a shield to atmosphere. Although not shown here, simulation was performed with heating at the cathode. This heat did not convect far into the plasma plume, 
therefore heating is truly by plasma processes and not by convection at the electrode. This is another limitation of CFD simulation of plasma plumes.

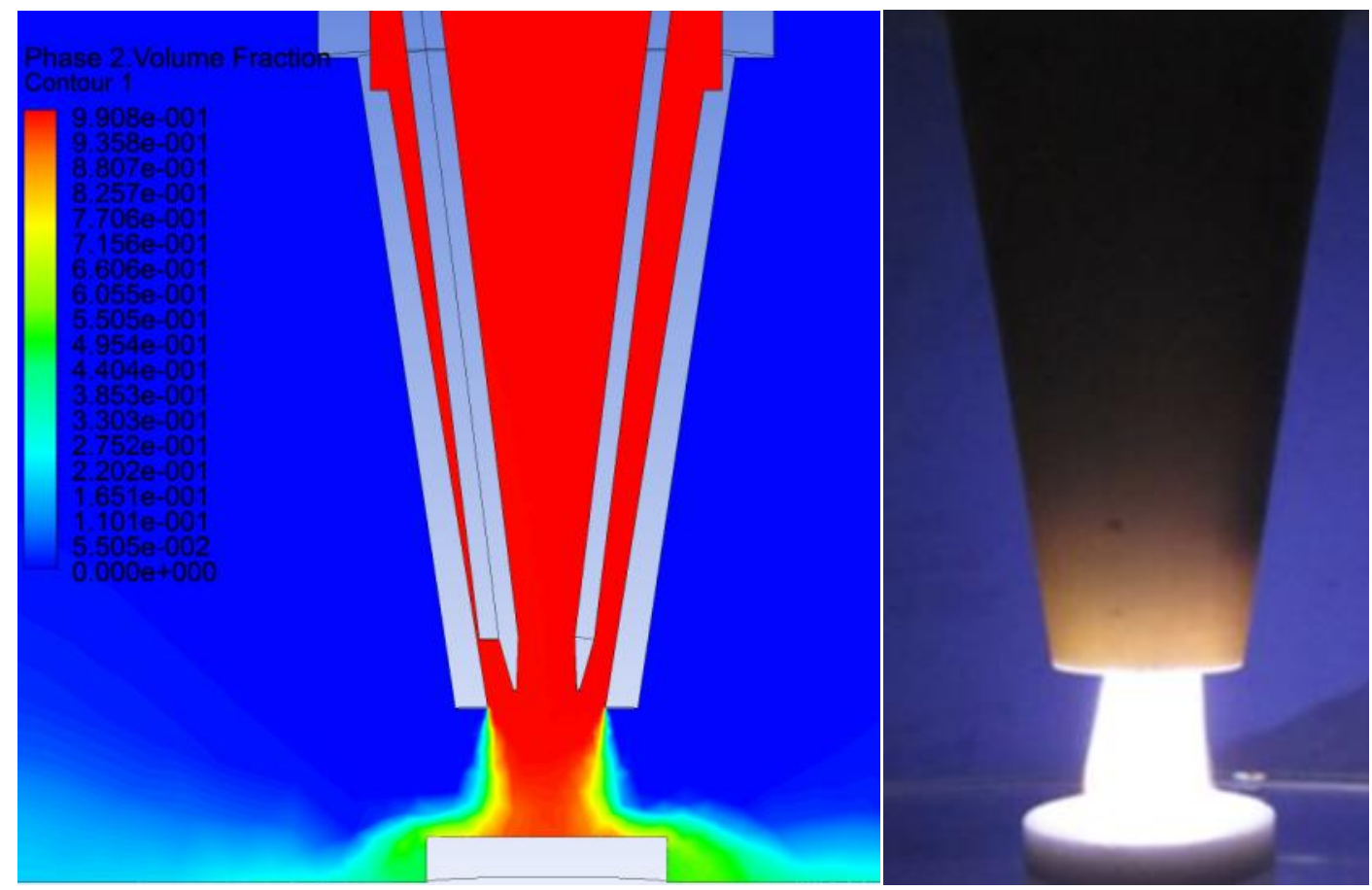

Figure 6.27. Volume fraction of argon dispersing through air at atmospheric pressure as simulated by computation fluid dynamics (left); a photo of the plasma brush (right).

CFD was also performed for the plasma brush with $\mathrm{O}_{2}$ addition at the ground electrode. Although oxygen is injected in the nozzle, argon concentration is similar to that of the pure argon plasma simulation, as shown in Figure 6.28 (left). The injected $\mathrm{O}_{2}$ does not penetrate well into the argon and the boundary layer effect remains, as shown in Figure 6.28 (right). Argon concentration is nearly identical to the previous case without oxygen addition. 

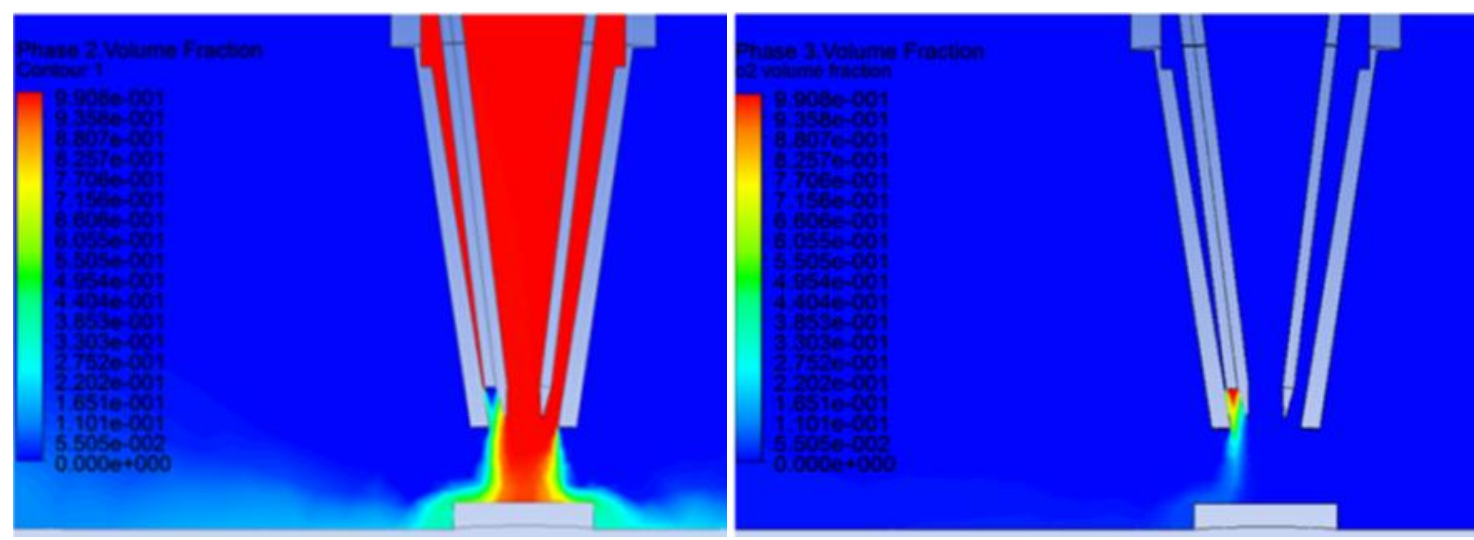

Figure 6.28. CFD simulation with $\mathrm{O} 2$ injected near the ground electrode; volume fraction of argon (left) and volume fraction $\mathrm{O} 2$ (right).

\subsection{Discussion}

SEM showed a layer of cell debris with underlying undamaged bacteria. Yang et al. had the same finding and described the lack of killing due to overlying cell debris blocking direct contact of plasma [6]. Our data suggests sterilization is not caused by oxidation. We came to this conclusion due to the following:

- Very little reactive oxygen species were created by the plasma in atmosphere and with injected oxygen as observed by OES;

- Sterilization of bacteria did not produce oxidation byproduct gases as observed by OES;

- CFD shows a boundary layer of argon reducing the probability of reactive oxygen species contacting the bacteria.

Our OES detector is not capable of detecting ozone created by the plasma. Although $\mathrm{O}_{3}$ is a strong oxidizer, Baysan et al. found little sterilization effect of oral bacteria [Baysan]. Also, other papers on sterilization by atmospheric plasma found ozone was not a major 
contributor. Therefore, we do not believe it is necessary to study further the creation of ozone in our plasma. Although we did not detect any oxidation byproduct gases with OES, concentrations may be sufficiently low outside our OES detection limit. Therefore, we do not accept our results as absolute proof of sterilization not being caused by oxidative species.

Yu et al suggest argon ions and excited neutral bombardment as a major sterilization contributor. Our plasma brush has two electrodes running parallel to each other with tips spaced the same distance apart from the nozzle opening. Plasma discharge occurs at the electrode tips. This causes an electric field acting transverse to the flow of argon, therefore the acceleration of ions and electrons should tend to have a direction transverse to the flowing argon. If the killing mechanism is thought to be due to bombardment by charged particles and accelerated neutrals, the directionality of the accelerating species may cause the charged species to skim across the surface of the samples. This would make penetration of plasma into lower lying cells more difficult and may explain the lack of sterilization of lower lying cells. The importance of direct contact of charged species on sterilization has been found elsewhere; Dobryinin et al. found the exclusion of charged species reduced the rate of sterilization by two orders of magnitude [15]. Sterilization by electron bombardment is dependent on the current density and velocity of electrons. Electrons freed during ionization are accelerated between the electrodes. Electrons will collide with substrates crossed by the electric field. The mean free path of an electron at atmospheric pressure is small; therefore the electron will impart much of its momentum to surrounding gas molecules. Most researchers conclude that the loss of momentum makes sterilization due to electron bombardment highly unlikely [24]. Similar to electron bombardment, sterilization by surface ablation is killing by impact of high temperature particles. Plasma particles, both charged 
and neutral, are accelerated fast enough to vaporize the cell membrane. Ablation has not been considered a major source of sterilization due to the low temperature, as described in a previous section, for dental plasma brushes. Although the bulk temperature of the plasma has been measured to be around body temperature, the non-equilibrium nature of the plasma causes high temperature gradients. The high temperature gradients may exist in such small volumes that our temperature probe would be too large to measure them apart from the bulk gas temperature. Therefore, very high temperature particles may be present in the plasma, but at low concentrations such that the bulk temperature remains low. These high temperature particles may be of high enough energy to destroy bacteria cell walls when they do come into contact with the cells.

Charged species could also play a major role in sterilization without bombardment. Charge accumulation may cause pore opening, i.e. electroporation, or instability in the bacteria cell wall which in itself may be enough to cause cell death [26,17]. Palanker et al. used atmospheric plasma electroporation for gene transfection [34]. Electroporation has historically suffered from the possibility of causing cell death. High electric fields can cause pores to enlarge to a point where the pore cannot be reclosed, i.e. irreversible electroporation, and eventual cell death [35]. Larousi suggests the sterilization mechanism may be a two part process: charge accumulation causing pore opening whereby reactive oxygen species may transport through cell walls with greater ease, followed by reactive oxygen species reacting with internal cellular structures causing permanent damage and eventual cell death. 


\subsection{Conclusion}

DC atmospheric plasma shows great promise in the sterilization of oral bacteria. The plasma brush killed high concentrations of bacteria in a matter of seconds. Plasma sterilization was found to not likely be caused by reactive oxygen species. Very few reactive oxygen species were observed by OES. Byproducts that would be formed during oxidation of bacteria were not observed by OES. The likelihood of reactive oxygen species reaching the bacteria is questionable due to the boundary layer of argon surrounding the substrate. This was observed during a CFD simulation. The authors suspect sterilization is caused by either of the two mechanisms:

- bombardment of charged species;

- accumulation of electrical charge.

In the former mechanism, ballistic charged and neutral species may rupture bacterial cell walls causing release of intracellular material and cell death. In the latter mechanism, accumulating electrons may cause opening of the cell wall, i.e. electroporation.

We suspect bombardment to be the greatest cause of sterilization because it would result in the abundant amount of destruction to bacteria as observed by SEM. Individual cells were nearly indistinguishable after treatment due to the great amount of destruction caused by the plasma. Lactobacillus and Streptococcus bacteria are gram positive and therefore have a thick cell membrane. Since sterilization is thought to be caused by bombardment, it is encouraging that plasma was capable of destruction of thick walled cells. This should mean faster sterilization when applied to thin walled gram negative bacteria. Clinical use of atmospheric pressure plasmas are limited by the small volume of plasma and slow sterilization rates. 


\subsection{References}

1. Featherstone J. The Continuum of Dental Caries-Evidence for a Dynamic Disease Process. Journal of Dental Research 2004; 83: C39-C42.

2. Yip H.K., Samaranayake L.P. Caries removal techniques and instrumentation. Clinical Oral Investigations 1998; 2: 148-154.

3. Yu Q.S., Huang C. Deactivation effects of atmospheric cold plasma brush. Applied Physics Letters 2006; 88: 013903.

4. Goree J., Liu B., Drake D., Stoffels E. Killing of S. mutans Bacteria Using a Plasma Needle at Atmoshperic Pressure. IEEE Transaction on Plasma Science 2006; 34: 1317-1324.

5. Sladek R., Stoffels E., Walraven R., Tielbeek P., Koolhoven R. Plasma Treatment of Dental Cavities: A Feasibility Study. IEEE Transaction on Plasma Science 2004; 32: 1540-1543.

6. Yang B., Chen J., Yu Q., Li H., Lin M., Mustapha A., Hong L., Wang Y. Oral bacterial deactivation using a low-temperature atmospheric argon plasma brush. Journal of Dentistry 2011; 39: 48-56.

7. Rupf S., Lehmann A., Hannig M., Schafer B., Schubert A., Feldmann U., Schindler, A. Killing of adherent oral microbes by a non-thermal atmospheric plasma jet. Journal of Medical Microbiology 2010; 59: 206-212.

8. Lu X., Cao Y., Yang P., Xiong Q., Xiong Z., Xian Y., Pan Y. An RC Plasma Device for Deactivation of Root Canal of Teeth. IEEE Transactions on Plasma Science 2009; 37: 668-673.

9. Huang C., Yu Q., Hsieh F., Duan Y. Bacterial Deactivation Using a Low Temperature Argon Atmospheric Plasma Brush with Oxygen Addition. Plasma Processes and Polymers 2007; 4: 77-87.

10. Yu Q., Huang C., Hsieh F., Huff H., Duan Y. Bacterial inactivation using a lowtemperature atmospheric plasma brush sustained with argon gas. Journal of Biomedical Materials Research Part B Applied Biomaterials 2007; 80: 211-219.

11. Lee H., Park G., Seo Y., Im Y., Shim S., Lee H. Modeling of atmospheric plasmas for biomedical applications. Journal of Physics D: Applied Physics 2011; 44: 1-27.

12. Larousi M., Akan T. Arc-Free Atmospheric Pressure Cold Plasma Jets: A review. Plasma Processes and Polymers 2007; 4: 777-788. 
13. Stark R., Schoenbach K. Direct current high-pressure glow discharges. Journal of Applied Physics 1999; 85: 2075-2080.

14. Larousi M. Low Temperature Plasma-Based Deactivation: Overview and State-ofthe-Art. Plasma Processes and Polymers 2005; 2: 391-400.

15. Dobrynin D., Fridman G., Friedman G., Fridman A. Physical and biological mechanisms of direct plasma interaction with living tissue. New Journal of Physics 2009; 11: 11-22.

16. Perni S., Shama G. Probing bactericidal mechanisms induced by cold atmospheric plasmas with Escherichia coli mutants. Applied Physics Letters 2007; 90: 073902.

17. Gaunt L.F., Beggs C.B., Georghiou G.E. Bactericidal Action of the Reactive Species Produced by Gas-Discharge Nonthermal Plasma at Atmospheric Pressure: A Review. IEEE Transaction on Plasma Science 2006; 34: 1257-1269.

18. Boudam M.K., Moisan M., Saoudi B., Popovici C., Gherardi N., Massines F. Bacterial spore inactivation by atmospheric-pressure plasma in the presence or absence of UV photons as obtained with the same gas mixture. Journal of Physics D: Applied Physics 2006; 39: 3494-3507.

19. Loesche W.J. Role of Streptococcus mutans in Human Dental Decay. Microbiological Reviews 1986; 50: 353-371.

20. Jay P. Lactobacillus acidophilus and dental caries. American Journal of Public Health 1938; 28: 759.

21. Madhwani T., McBain A. Bacteriological effects of a Lactobacillus reuteri probiotic on in vitro oral biofilms. Archives of Oral Biology 2011; 56: 1264-1273.

22. Smith A.M., Bowen W.H. In situ studies of pellicle formation on hydroxyapatite discs. Archives of Oral Biology 2000; 45: 277-291.

23. Jiang C., Schaudinn C., Jaramillo D., Webster P., Costerton J.W. In Vitro Antimicrobial Effect of a Cold Plasma Jet against Enterococcus faecalis Biofilms. ISRN Dentistry 2012; 2012: 1-6.

24. Koulik P., Begounov S., Goloviatinskii S. Atmospheric Plasma Deactivation and Deodorization of Dielectric Surfaces. Plasma Chemistry and Plasma Processing 1999; 19: 311-326.

25. Keudell A., Awakowicz P., Benedikt J., Raballand V., Yanguas-Gil A., Opretzka C., et al. Inactivation of Bacteria and Biomolecules by Low-Pressure Plasma Discharges. Plasma Processes and Polymers 2010; 7: 327-352. 
26. Laroussi M., Lu, X. Room-temperature atmospheric pressure plasma plume for biomedical applications. Applied Physics Letters 2005; 87: 113902.

27. Stoffels E., Flikweert A.J., Stoffels W.W., Kroesen G.M. Plasma needle: a nondestructive atmospheric plasma source for fine surface treatment of (bio)materials. Plasma Sources Science and Technology 2002; 11: 383-388.

28. Jiang C., Vernier P.T., Chen M.T., Wu Y.H., Wang L.L., Gundersen M.A. Low Energy Nanosecond Pulsed Plasma Deactivation for Endodontic Applications. IEEE International Power Modulators and High Voltage Conference, Proceedings of the 2008 2008; :27-31.

29. Montie T.C., Wintenberg K.K., Roth J.R. An Overview of Research Using the One Atmosphere Uniform Glow Discharge Plasma (OAUGDP) for Deactivation of Surfaces and Materials. IEEE Transactions on Plasma Science 2000; 28: 41-50.

30. Kunhardt E.E. Generation of Large-Volume, Atmospheric-Pressure, Nonequilibrium Plasmas. IEEE Transactions on Plasma Science 2000; 28: 189-200.

31. Vujosevic D., Mozetic M., Cvelbar U., Krstulovic N., Milosevic S. Optical Emission Spectroscopy Characterization of Oxygen Plasma During Oxygen Plasma Deactivation of Escherichia Coli. Journal of Applied Physics 2007; 101: 103305.

32. Sakiyama, Y., Graves, D., Stoffels, E., 2008, "Influence of electrical properties of treated surface on RF-excited plasma needle at atmospheric pressure," Journal of Physics D: Applied Physics, 41, 1-9.

33. Baysan A., Beighton D., Assessment of the Ozone-Mediated Killing of Bacteria in Infected Dentine Associated with Non-Cavitated Occlusal Carious Lesions. Caries Research 2006; 41: 337-341.

34. Palanker D., Chalberg T., Vankov A., Huie P., Molnar F., Butterwick A., et al. Plasma-Mediated Transfection of RPE. Proceedings SPIE Ophthalmic Technologies XVI 2006; 6138: 1-9.

35. Rubinsky B. Irreversible Electroporation in Medicine. Technology in Cancer Research and Treatment 2007; 6: 255-259. 


\title{
Chapter 7 Disinfection of Oral Bacteria on Dentin by
}

\author{
Atmospheric Pressure Plasma
}

\begin{abstract}
Objective: This research was to investigate the plasma treatment effects on oral bacteria seeded on dentin.

Methods: A direct current (DC) powered atmospheric pressure plasma brush was used to disinfect two different Gram-positive oral bacteria. Oral bacteria Lactobacillus acidophilus and Streptococcus mutans were seeded on dentin slices and their survivability was examined by the pour plate method after varying durations of plasma exposure. Scanning electron microscopy (SEM) was performed to examine the location of cell lysis. Bacteria were also seeded on glass slides held apart to create a microchannel and plasma treatment was performed with electrodes both transverse and in-line to the microchannel. SEM was again performed to examine the location of cell lysis.

Results: Major destruction of bacteria was observed, but full disinfection was not achieved. Plasma effectively deactivated on average $75 \%$ of L. acidophilus and $85 \%$ of S. mutans after 15 seconds. SEM examination confirms intact bacteria within dentinal tubules seemingly unaffected by plasma treatment. Plasma treatment is only capable of penetrating microchannels when treated in-line with microchannels.

Conclusions: Atmospheric pressure plasma may be an effective method for the disinfection of oral bacteria. Bacteria may survive in dentin tubules, but these are considered negligible to the long term success of the restoration.
\end{abstract}




\subsection{Introduction}

Tooth decay in all forms has in common the demineralization of calcium and phosphate by acids [1]. Acid is produced by oral bacteria during fermentation of carbohydrates from residual food particles. If demineralization continues, a cavity may form, further exacerbating the problem due primarily to lowered efficacy of mechanical action. Once cavitation reaches the dentinal layer, bacteria may invade the dentin tubules producing tubular sclerosis known as dead tracts. It is therefore desirable to develop a method to effectively disinfect bacteria on tooth surfaces as well as bacteria in dentin tubules. Use of antibiotics can almost completely prevent caries progression, but treatment is not immediate [2]. Further, the success of an antibiotic depends on the bacterium and its possible resistance to the antibiotic. A variety of bacteria may be present in the mouth while the antibiotics available may not be effective on all bacteria present [3]. Also, overuse of antibiotics has led to widespread multi-antibiotic resistance [4]. Thus, it is imperative to develop a method to deactivate oral bacteria quickly and without specificity.

In the previous chapter, the killing effect of a direct current (DC) atmospheric plasma brush was examined on an enamel analogue, i.e., hydroxyapatite (HA). To further examine the clinical relevance of the plasma brush, disinfection of bacteria on human dentin was studied and reported in this article. Lactic, acetic, formic, and propionic acids all dissolve tooth mineral and are known to be formed by the common Gram-positive oral bacteria Lactobacillus acidophilus and Streptococcus mutans [4]. In this study, L. acidophilus and S. mutans were seeded on dentin slices and plasma treated in order to examine the effectiveness of plasma treatment for caries prevention and disinfection before caries restoration. Also 
included in the previous chapter was evidence that disinfection was mainly due to bombarding energetic species. This study further examined this mechanism of disinfection.

\subsection{Materials and Methods}

\subsubsection{Bacteria preparation}

Bacterial cultures were obtained and inocula created as stated in the previous chapter. Un-erupted human third molars were collected under a protocol approved by the University of Missouri-Kansas City Adult Health Sciences Institutional Review Board and after obtaining patient consent. All tissue samples were non-patient identified and all samples were handled and disposed of according to the protocols suggested by the Department of Environmental Health and Safety at the University of Missouri. The teeth had no caries and were stored in PBS (pH 7.4) containing $0.02 \%$ sodium azide to inhibit bacterial growth. Dentin slices were cut with a diamond saw into slices with diameters of $9.5 \mathrm{~mm}$ diameter and around $3 \mathrm{~mm}$ thickness. The dentin surface was exposed by removing the crown and then wet sanded with 200 and 600 grit sandpaper. Dentin slices were stored in distilled peptone water for 48 hours to remove the sodium azide. Dentin slices were etched with $3 \mathrm{M}$ brand phosphoric acid etchant for 15 seconds as directed and rinsed by vortex mixing in $10 \mathrm{~mL}$ peptone water. Dentin slices were air dried with compressed air for 30 seconds. Five microliters of the inoculum was pipetted and spread onto the surface then air dried in a biosafety hood for seven minutes. A similar shiny wet appearance was present after seven minutes drying compared to five minutes drying of HA discs used in the previous chapter. The plasma plume only contacted around $1 / 6$ of the entire surface at any given time, hence equivalent treatment times are therefore reported as $1 / 6$ the total sample treatment time. 
Plasma treatment, bacterial enumeration, and SEM specimen preparation was the same as described in the previous chapter.

\subsubsection{Treatment of bacteria seeded on microchannel walls}

To better understand the mechanisms of disinfection, L. acidophilus was spread on two $1 " \times 1$ " glass slides near the glass slides' edges. The slides were kept parallel to each other, the edges were aligned, and the slides were separated forming a microchannel around $80 \mu \mathrm{m}$ wide. The plasma plume protrudes from the plasma brush creating a thin blade shape spanning the gap between the needle electrodes. The bacteria seeded in the microchannel were plasma treated in two configurations: with electrodes positioned transverse to the microchannel and with electrodes positioned in-line with the microchannel, as shown in Figure 7.1 (left) and Figure 7.1 (right), respectively. 


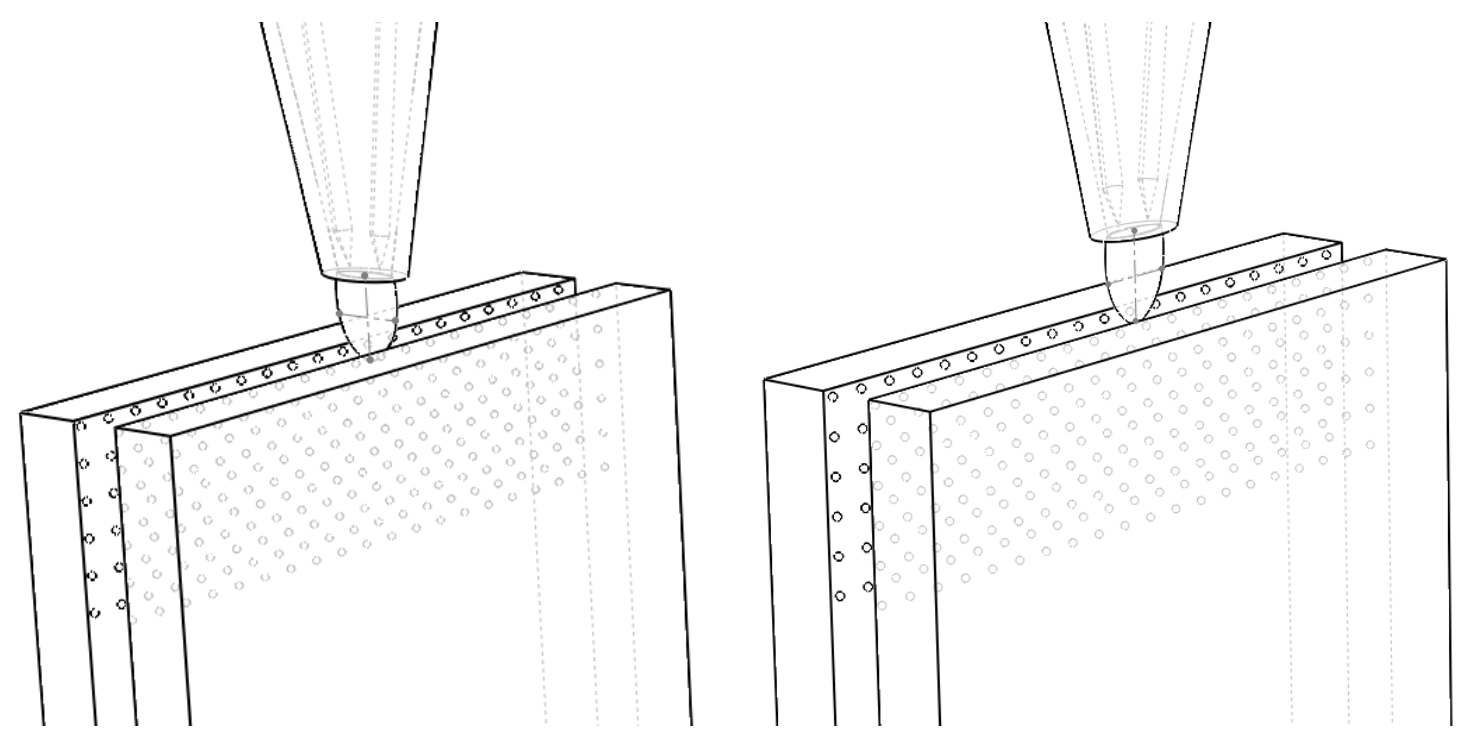

Figure 7.1. A depiction of the microchannel in relation to the plasma plume (not to scale) when treated with electrodes transverse to the microchannel (left) and in-line with the microchannel (right); bacteria represented as spheres.

\subsection{Results and Discussion}

Deactivation rates on dentin of L. acidophilus and S. mutans are compared to deactivation rates on HA discs, shown in figures 7.2-7.3, respectively. Untreated samples start at slightly higher concentrations for dentin samples than with HA. This is most likely due to the hydration state of the samples. Although both surfaces looked to be at a similar state of dryness, dentin tubules stayed wet compared to HA due to capillary effect and therefore bacteria did not die from drying. Plasma effectively deactivated on average $75 \%$ of L. acidophilus and $85 \%$ of S. mutans after 15 seconds, but a majority of the killing was in the first three to five seconds. At five seconds, the deactivation rate starts to plateau and full disinfection was not achieved. To determine the location of the surviving bacteria, SEM was performed as shown in figures 7.4-7.6. Figure 7.4 shows untreated bacteria present on the surface of the dentin. Penetration of S. mutans into tubules is very apparent. Figure 7.5 shows extensive damage to surface bacteria. SEM was also performed on cross sections of 
dentin that were snapped in half after treatment. Figure 7.6 shows that even after 15 seconds of plasma treatment, both L. acidophilus and S. mutans remained intact within dentin tubules.

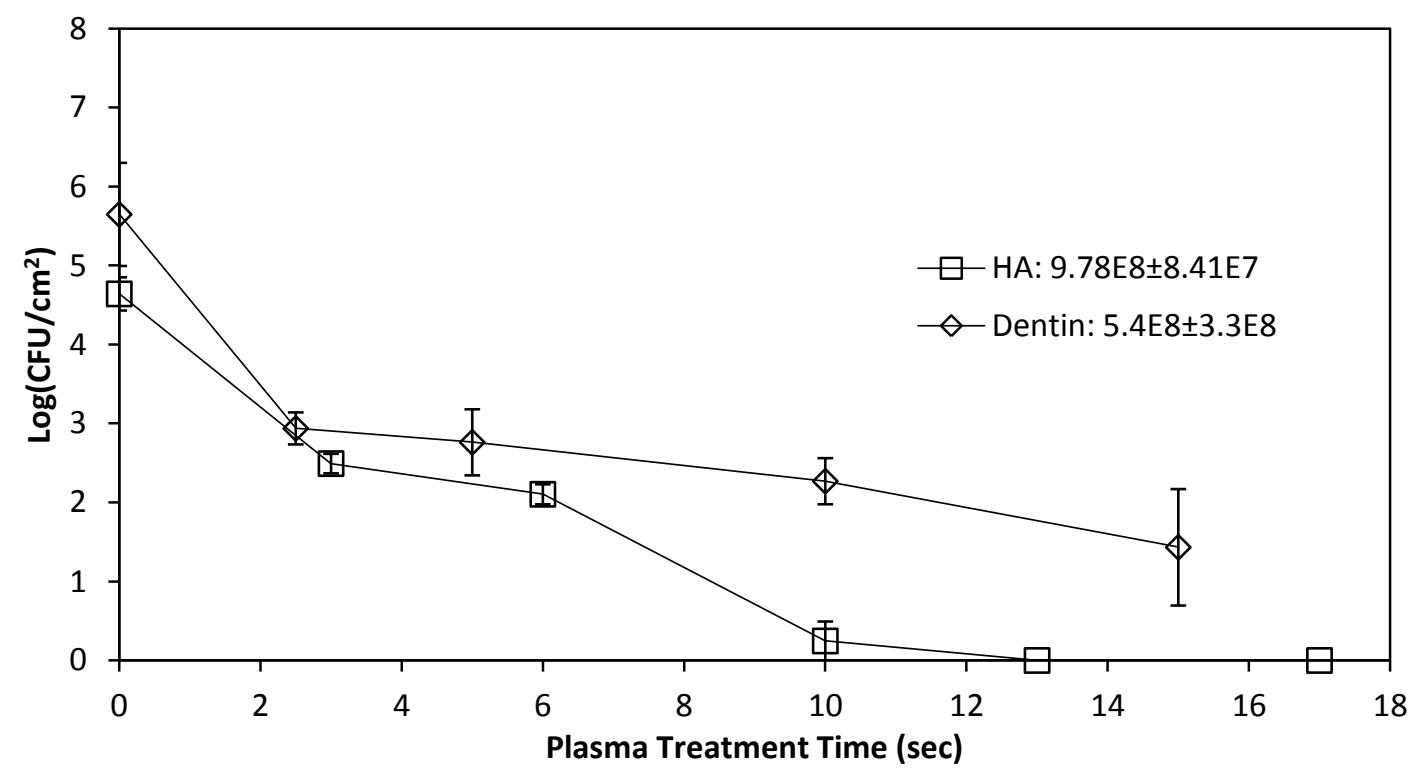

Figure 7.2. Plasma deactivation effect on L.acidophilus seeded on dentin and HA with initial cell densities shown in the legend $(\mathrm{cfu} / \mathrm{mL})$.

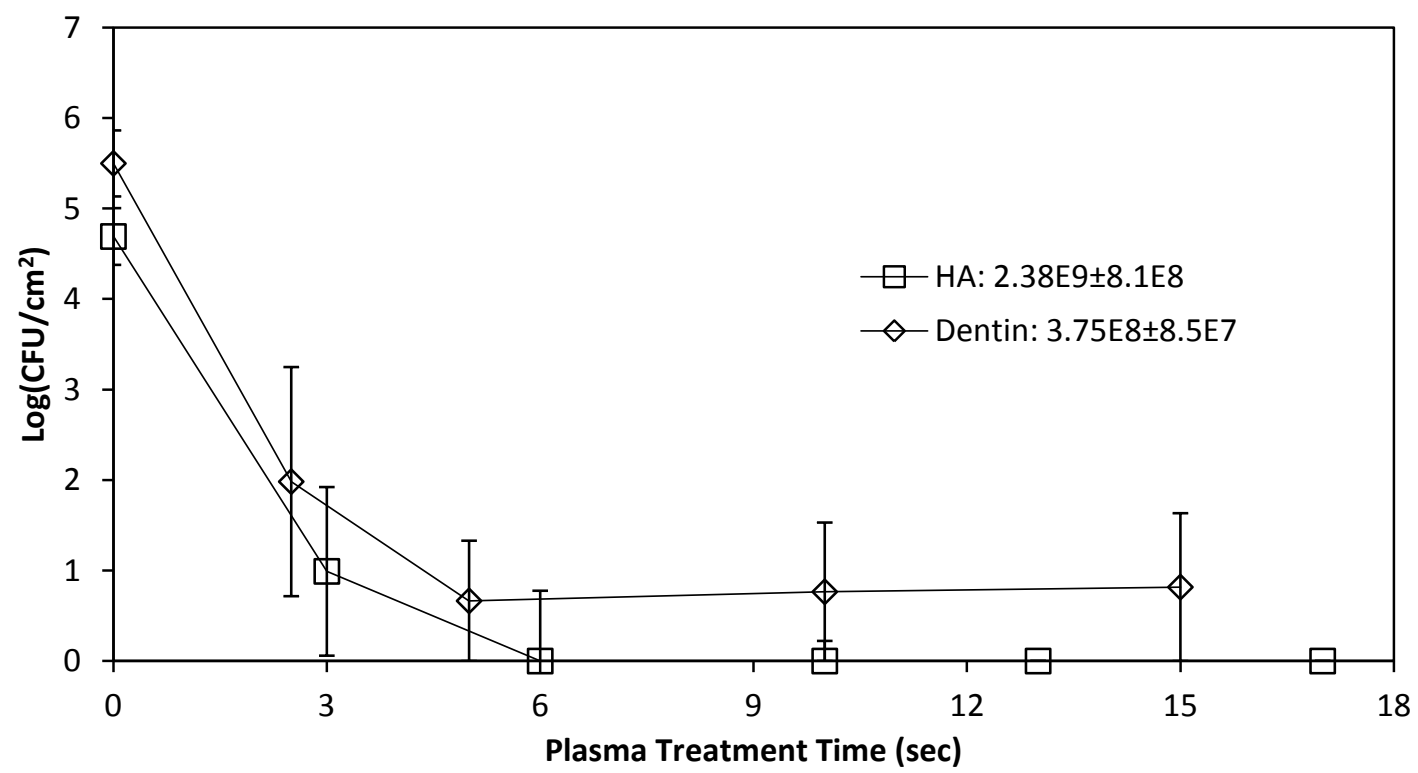

Figure 7.3. Plasma deactivation effect on S. mutans seeded on dentin and HA with initial cell densities shown in the legend $(\mathrm{cfu} / \mathrm{mL})$. 
In previous chapter, it was also hypothesized that if bombardment was the mechanism of deactivation, that the trajectory of the bombarding species would tend to be perpendicular to the gas flow. This is due to the nature of the plasma; ions and electrons are accelerated by the electric field created between the two needle electrodes. To investigate this theory, we tested whether a direction-dependent killing effect could be observed determined by the substrate geometry, e.g. plasma treatment was performed on bacteria placed along the walls of a microchannel. When the plasma plume was oriented such that the electric field was transverse to the microchannel, there was no apparent disinfecting effect on microchannel faces, as shown in Figure 7.7. When the plasma plume was oriented such that the electric field was in-line with the microchannel, there was a disinfection effect that penetrated around $0.07 \mathrm{~mm}$ into the microchannel, as shown in Figure 7.8.
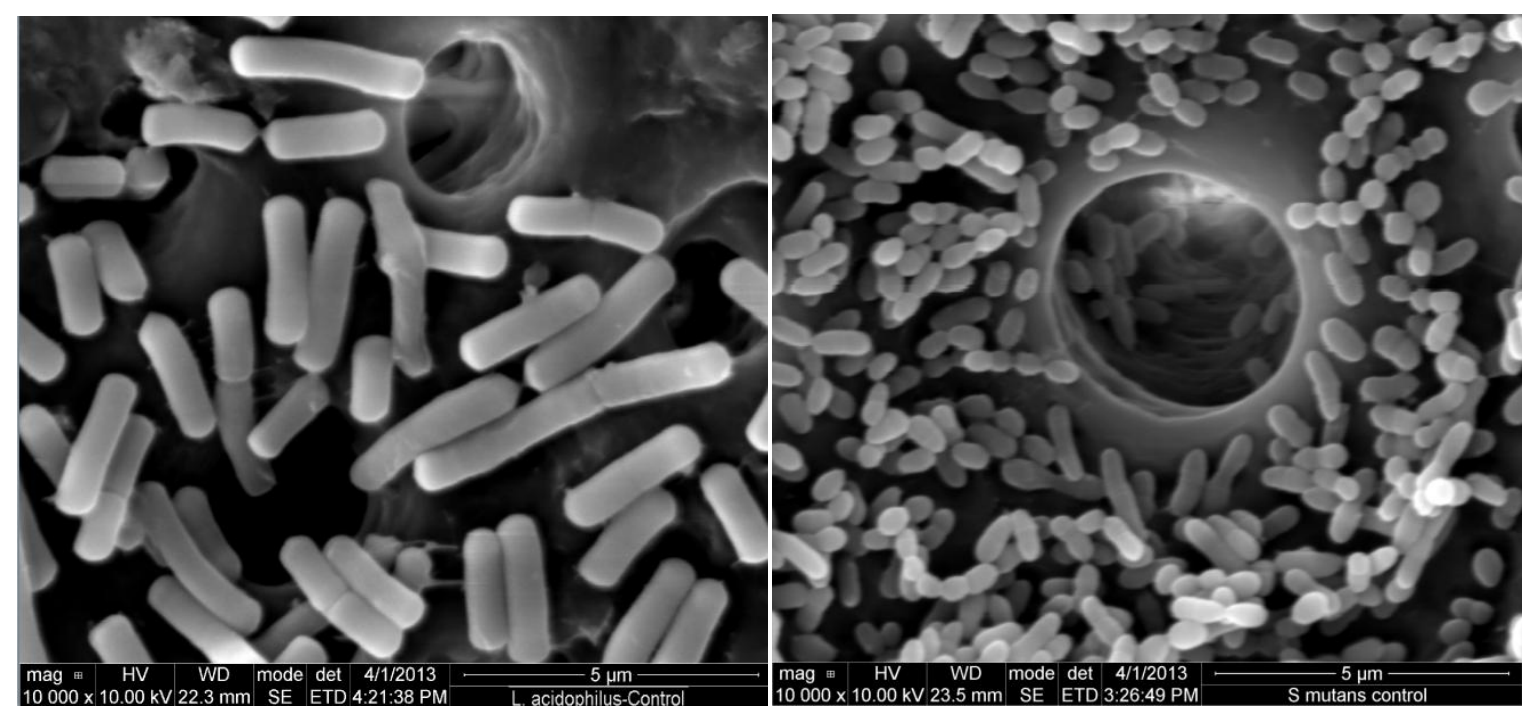

Figure 7.4. SEM of dentin surfaces seeded with L. acidophilus (left) and S. mutans (right) before plasma treatment. 

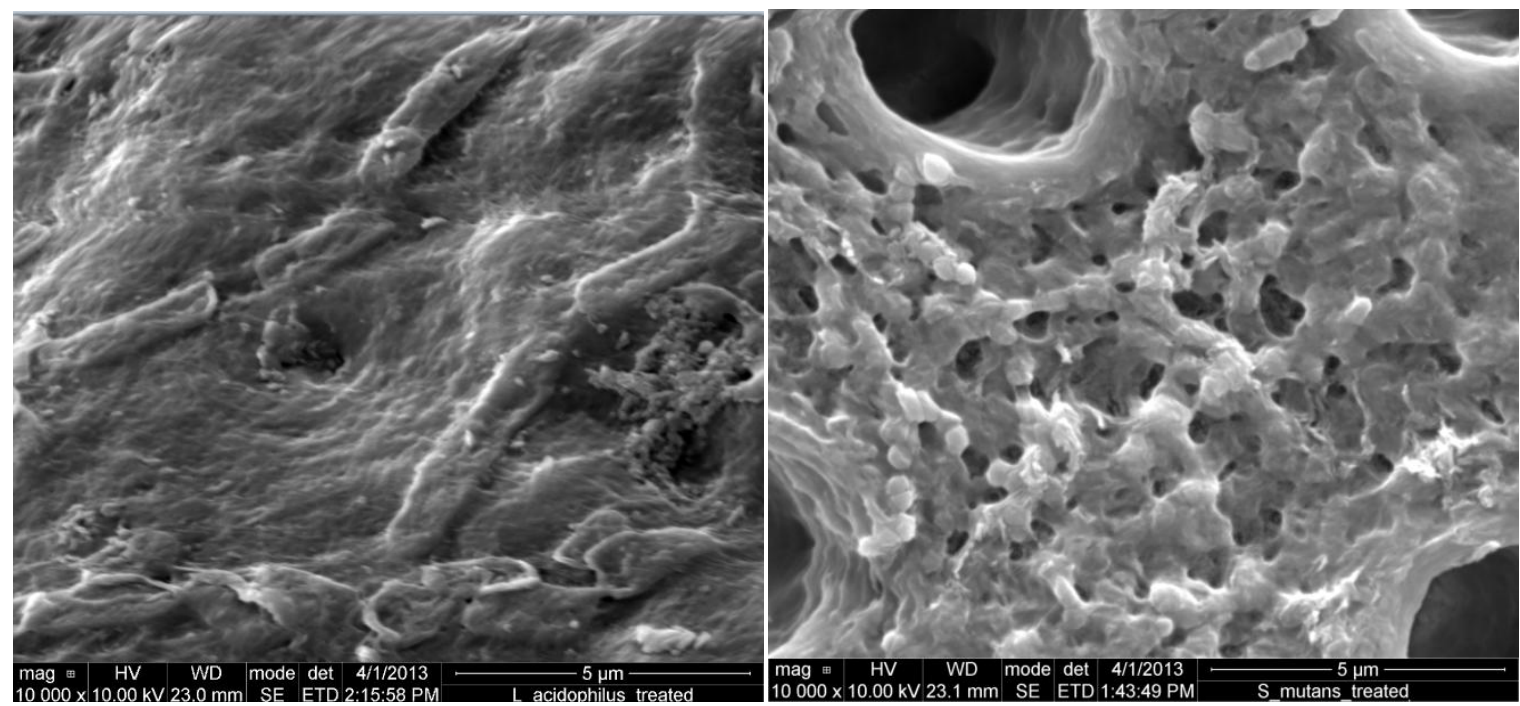

Figure 7.5. SEM of dentin surfaces seeded with L. acidophilus (left) and S. mutans (right) after 15 seconds plasma treatment.
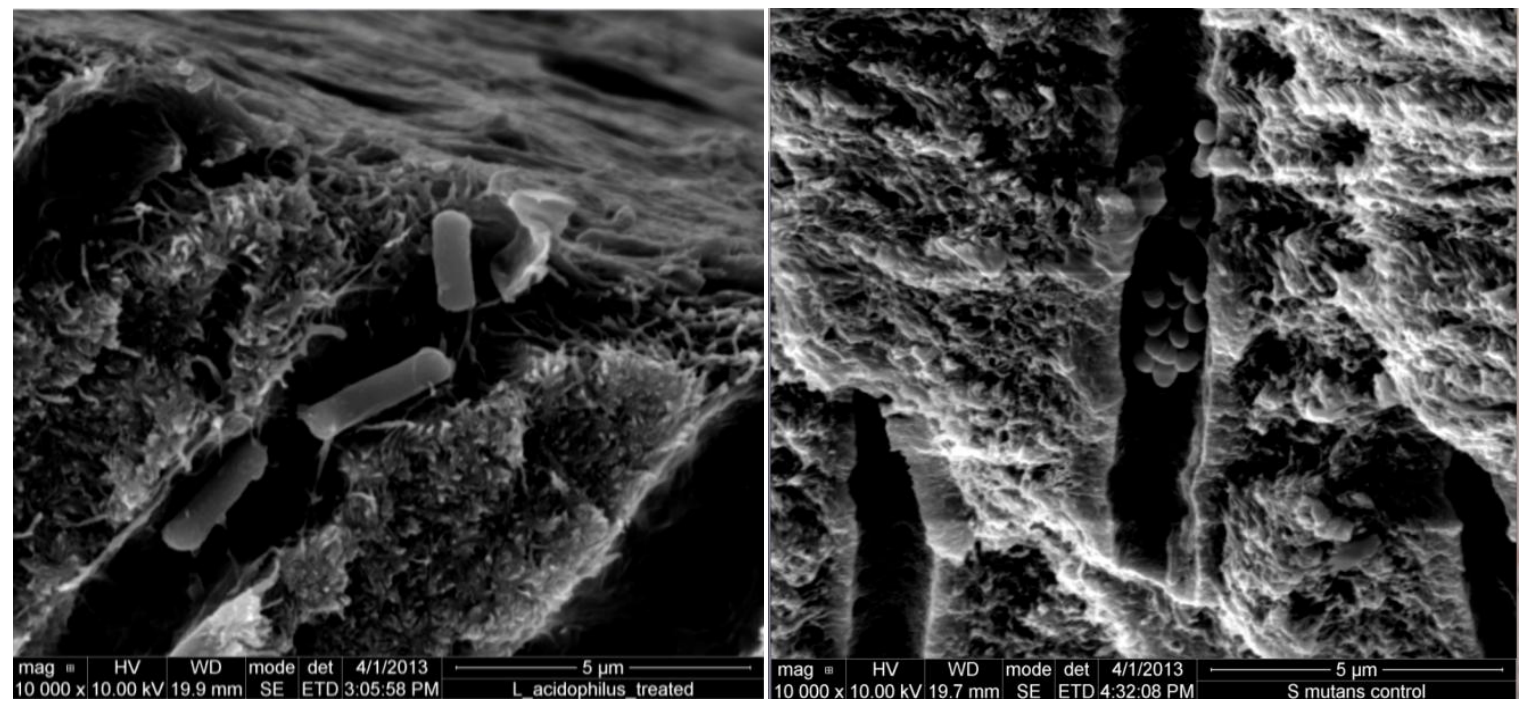

Figure 7.6. SEM cross-section views of dentin seeded with L. acidophilus (left) and $S$. mutans (right) after 15 seconds plasma treatment. 

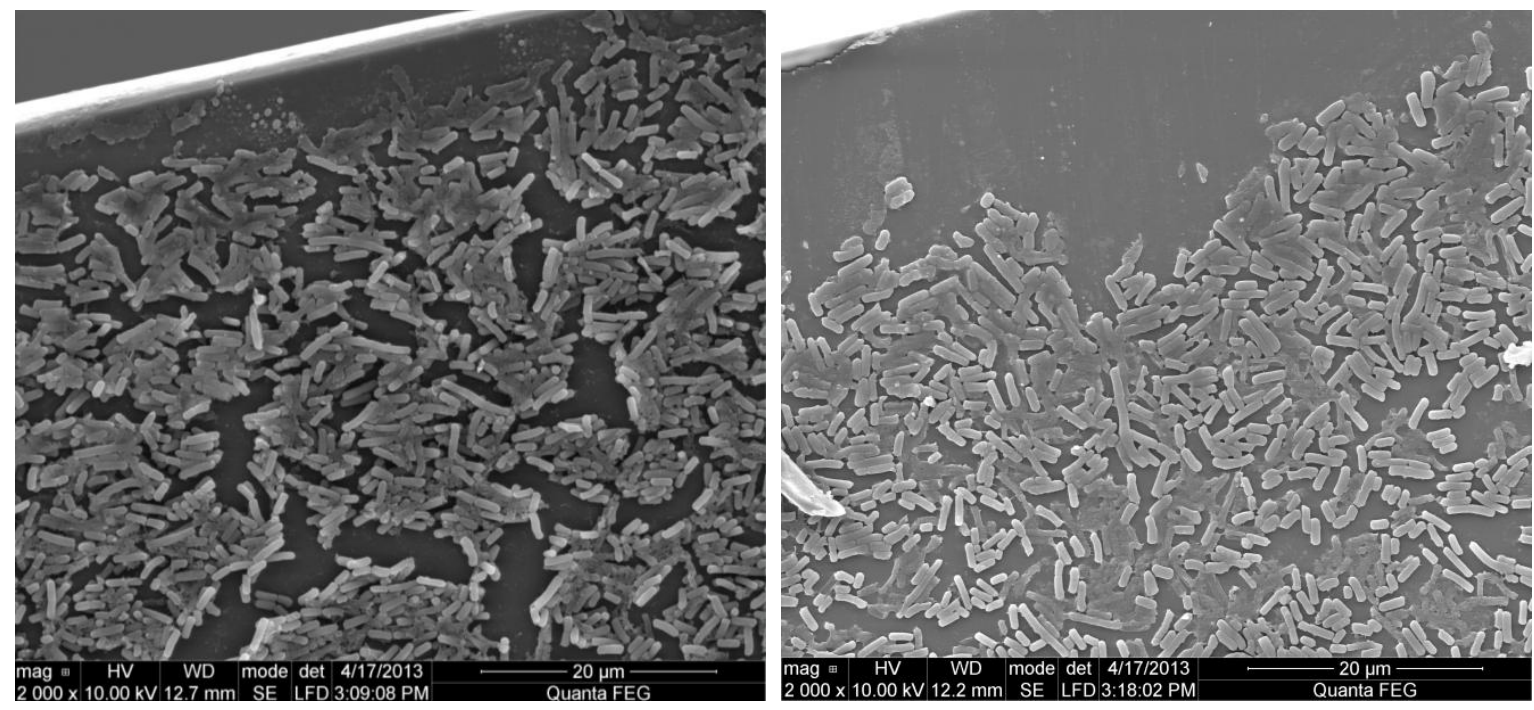

Figure 7.7. SEM of L. acidophilus seeded on glass cover slips after plasma treatment with electrodes transverse to the microchannel; anode (left) and cathode (right); top of microchannel upper left.
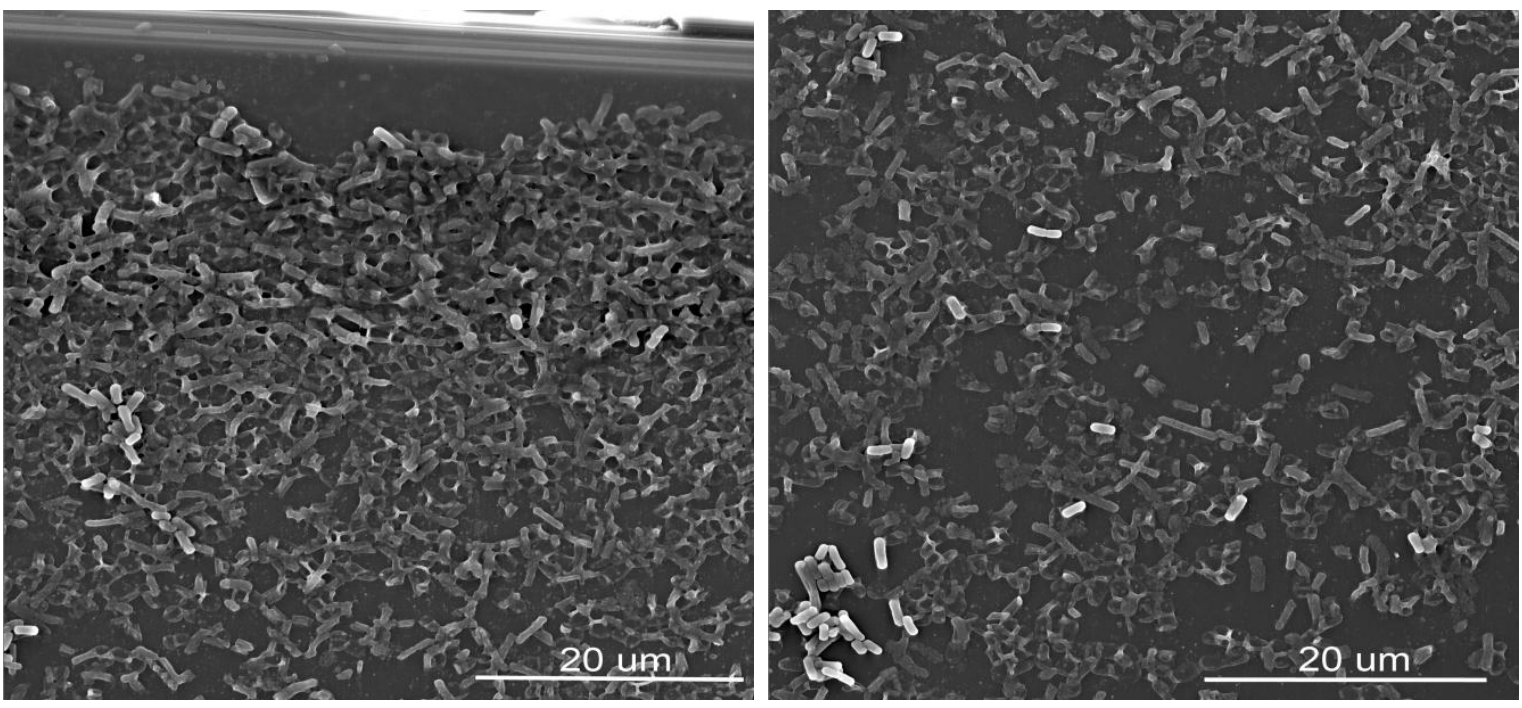

Figure 7.8. SEM of L. acidophilus seeded on glass cover slips after plasma treatment with electrodes in-line with the microchannel; near the surface (left) and $0.064 \mathrm{~mm}$ from the surface (right).

In contrast to treatment on HA, plasma treatment on dentin was not as effective. In

the previous chapter, there was an observed shadowing effect caused by cell debris blocking lower lying bacteria from plasma treatment when high areal seeding concentrations were 
applied. In this study, the areal seeding concentration was kept low enough to obtain full disinfection on HA and therefore it should be assumed that no shadowing took place with the use of dentin slices. Instead, live bacteria must remain in the tubules alone. Rupf et al. (2010) found a similar decrease in deactivation rate when compared to treatment on agar and they proposed bacteria were penetrating into deep dentin and therefore were not close enough to the plasma source to be treated [5]. Treatment of L. acidophilus caused much debris that seems to be blocking the tubules, as shown in Figure 7.5, but debris from treated $S$. mutans does not look to be blocking tubules. Therefore, the lack of full disinfection of S. mutans cannot be due to tubule blockage. SEM examination confirmed intact bacteria within dentin tubules. These were assumed to be live cells since they remained intact compared to the extensive destruction of cells at the dentin surface. Plasma was unable to travel deep within the tubule to affect deep lying bacteria.

The observed killing effect within a microchannel further supports this theory. The microchannel used in this experiment is much larger than dentin tubules, yet plasma was still ineffective when applied transverse to channel. The ends of the microchannel were open allowing gas to flow freely. If reactive oxygen species (ROS) were created by the plasma, these should be able to flow through the channel without regard to the orientation of the plume, yet cell destruction was only observed when the plume was oriented in-line to the microchannel. This evidence supports the hypothesis that ROS's are not the major cause of disinfection. Instead, deactivation tends to be more based on line-of-sight which suggests bombardment as the major deactivation mechanism. Bombarding species accelerated perpendicular to the microchannel may only skim across the surface without penetration. 
Since it has been determined that the current plasma brush design is unable to deactivate bacteria within dentin tubules, the next question is whether or not these remaining bacteria will cause further harm to the tooth during caries prevention and what would be the fate of a caries restoration with remaining tubule bacteria. Caries are not necessarily doomed to continued sclerotic progression without clinical restoration. Naturally, minerals from saliva may deposit a new veneer effectively repairing lesions [Featherstone]. Over time, mineral will deposit on tubule walls causing tapering and even complete closure of tubules. Dentine tubules are 0.9-2.5 $\mu \mathrm{m}$ in diameter. Diameters of oral streptococci average 0.5-0.7 $\mu \mathrm{m}$ and lactobacilli average 0.6-0.9 $\mu \mathrm{m}$ [6]. The natural closing of tubules restricts further bacterial penetration and nutrients necessary for bacteria survival. If a clinical restoration has been deemed necessary, bacteria remaining in dentin tubules may not mean the failure of the restoration. Studies have found that it is not bacteria within the dentin tubules which cause lesion progression, but instead, it is the biofilm at the cavity surface [7-9]. Thompson et al. (2008) surveyed 23 studies on the subject of partial removal of deep carious lesions and found that bacteria either die or do not cause lesion progression [10]. Thus, if the biofilm is removed and the cavity sealed, the lesion will not spread further. Superficial bacteria entombed by a filling will not have any deleterious effects. Sealing is thought to arrest lesion progression due to the lack of nutrients present for bacteria to metabolize. Also, the tubule environment is generally lacking in free oxygen further limiting the survivability of aerobic lactobacilli and streptococci. It is true that a composite restoration will shrink during polymerization, but most composite restorations incorporate a thin liner or adhesive layer between the composite and tubule [11,12]. Indeed, sealing with a liner is nearly water 
impenetrable [13]. Therefore, it is not necessary for plasma treatment to penetrate dentin tubules in order to achieve adequate caries restoration.

\subsection{Conclusion}

Extensive and rapid disinfection of oral bacteria was achieved with an atmospheric pressure plasma brush. Live bacteria remained after treatment likely surviving in the dentin tubules which provided shielding from bombarding energetic species. Although full disinfection was not achieved, the remaining bacteria are considered negligible to the success of the restoration as long as tubules are kept isolated from the environment by a restorative adhesive liner. When employed in this manner, the plasma brush may be effective at disinfecting bacteria, thus lessening the need for removal of bacteria and sclerotic tissue by drilling. Less drilling would result in less pulpal cavity exposure, reducing the likelihood of sensitivity. A direction-dependent killing effect was observed, suggesting deactivation of bacteria was caused by bombarding species. Although bombardment would suggest a high plume temperature, the temperature as measured by a fiber optic thermometer does not go beyond $45^{\circ} \mathrm{C}$. Indeed, plasma does not cause pain when applied to these researchers skin for prolonged exposure times. It is suggested that the high velocity particles comprised a small proportion of the entire plume and therefore a low bulk temperature remained. The inability of the plasma to penetrate deep into the developed microchannel brings into question its effectiveness for treatments into small crevices. The performance of the plasma may be dependent on the geometry of the cavity in question. 


\subsection{References}

1. Featherstone, J., The Continuum of Dental Caries-Evidence for a Dynamic Disease Process. Journal of Dental Research 2004, 83, C39-C42.

2. Gibbons, R., Van Houte, J., Dental Caries. Annual Review of Medicine 1975, 26, 121136.

3. Villedieu, A., Diaz-Torres, M., Hunt, N., McNab, R., Spratt, D., Wilson, M., Mullany, P., Prevalence of Tetracycline Resistance Genes in Oral Bacteria. Antimicrobial Agents and Chemotherapy 2003, 47, 878-882.

4. Walker, C., The acquisition of antibiotic resistance in the periodontal microflora. Periodontology 2000, 10, 79-88.

5. Rupf, S., Lehmann, A., Hannig, M., Schafer, B., Schubert, A., Felmann, U., Schindler, A., Killing of adherent oral microbes by a non-thermal atmospheric plasma jet. Journal of Medical Microbiology 2010, 59, 206-212.

6. Love, R., Jenkinson, H., Invasion of Dentinal Tubules By Oral Bacteria. Critical Reviews in Oral Biology \& Medicine 2002, 13, 171-183.

7. Kidd, E., How 'Clean' Must a Cavity Be before Restoration? Caries Research 2004, 38, 305-313.

8. Oliveira, E., Carminattia, G., Fontanella, V., Maltz, M., The monitoring of deep caries lesions after incomplete dentine caries removal: results after 14-18 months. Clinical Oral Investigations 2006, 10, 134-139.

9. Mertz-Fairhurst, E., Curtis, J., Ergle, J., Rueggeberg, F., Adair, S., Ultraconservative and Cariostatic Sealed Restorations: Results At Year 10. The Journal of the American Dental Association 1998, 129, 55-66.

10. Thompson, V., Craig, R., Curro, F., Green, W., Ship, J., Treatment of deep carious lesions by complete excavation or partial removal: A critical review. The Journal of the American Dental Association 2008, 139, 705-712.

11. Zeiger, D., Sun, J., Schumacher, G., Lin-Gibson, S., Evaluation of dental shrinkage and leakage in extracted teeth using X-ray microcomputed tomography. Dental Materials 2009, 25, 1213-1220.

12. Brannstrom, M., Nyborg, H., Pulpal reaction to composite resin restorations. The Journal of prosthetic dentistry 1972, 27, 181-189. 
13. Eriksen, H., Protective effect of different lining materials placed under composite resin restorations in monkeys. Scandanavian journal of dental research 1974, 82, 373-380. 


\section{Chapter 8 Conclusions and Future Prospects}

This dissertation set out to investigate plasma processing in the areas of corrosion and hydrogen re-embrittlement resistant coatings as well as to study the effectiveness and mechanisms of sterilization by atmospheric plasmas. In this final chapter,

the research contributions of this dissertation and directions for future

research will be reviewed.

\subsection{Introduction}

The following are the main research contributions provided by this dissertation.

Plasma coating provided hydrogen re-embrittlement resistance greater than chromate conversion coating, the industry standard, due to an increase in corrosion resistance (Chapter 2). In contrast to electroplating of passivating metals, plasma coatings were not found to cause hydrogen embrittlement. The plasma coating is an environmentally benign alternative to the toxic chromate conversion coatings previously used in industry.

A new hybrid coater was constructed combining the plasma coating and ion vapor deposition processes into one chamber (Chapters $4 \& 5$ ). Combining both processes in one reactor was not found to contaminate either coating as determined by energy dispersive spectroscopy and glass bead peen adhesion testing.

A plasma coating was produced in the hybrid reactor that effectively improved the corrosion resistance of aluminum high strength steel covered by ion vapor deposited aluminum as 
determined by potentiodynamic polarization testing and salt fog spray testing (Chapter 5). The finding might mean the removal of the electroplating process from aircraft production. This would mean the elimination in direct electroplating process costs, baking out of hydrogen, and associated water treatment costs with little costs incurred by adding the plasma coating process

The oxide interface was found key to good corrosion resistance (Chapter 5). Oxygen bridged between ion vapor deposited aluminum and plasma coating to provide strong adhesion, and allowed the aluminum to form a passivation layer which defended against corrosion.

An atmospheric plasma brush was constructed that created a plasma at safe temperatures and was compact enough for use in the mouth for oral plasma treatments (Chapter 6).

Rapid sterilization of the common oral bacteria L. acidophilus and S. mutans was achieved on hydroxyapatite discs. A plasma exposure time of 13 seconds effectively killed all bacteria when concentrations were less than $6.9 \times 10^{6} \mathrm{cfu} / \mathrm{cm}^{2}$ for L. acidophilus and $1.7 \times 10^{7} \mathrm{cfu} / \mathrm{cm}^{2}$ for S. mutans. At higher bacterial concentrations of $1.2 \times 10^{8}-2.5 \times 10^{8} \mathrm{cfu} / \mathrm{cm}^{2}$, a $1.5 \log$ reduction in bacterial concentration was observed in the first 7 seconds, but nearly no further reduction in bacterial population was achieved after 20 seconds. Upon examination by scanning electron microscopy, sterilization was observed to occur on top layer bacteria. Sterilization occurred faster than most other plasma sources found in literature and other chemical methods deemed safe for oral use.

Reactive oxygen species created in the plasma were not considered to be the main cause of sterilization. Bacteria that were plasma treated in an oxygen deprived jar were found to be 
sterilized at the same rate as in atmosphere. Few reactive oxygen species were observed in the plasma by optical emission spectroscopy. Addition of pure oxygen into the plasma did not increase the sterilization rate. No carbon or nitrogen containing species, indicative of oxidation, were observed in the plasma by optical emission spectroscopy during plasma treatment.

Rapid sterilization on dentin of the common oral bacteria L. acidophilus and S. mutans was achieved (Chapter 7). Killing rates on dentin were similar as that found with hydroxyapatite. Plasma effectively deactivated on average $99.97 \%$ of L. acidophilus and S. mutans after 15 seconds, but sterilization was increased by further treatment. Scanning electron microscopy provides strong evidence that live bacteria remain in dentin tubules.

Plasma sterilization in a microchannel showed evidence that killing is due to some line-ofsight mechanism (Chapter 7). The killing effect of plasma was dependant on the orientation of the plasma plume with respect to the microchannel. Plasma treatment with the plume inline with the microchannel coated with bacteria caused excessive cell damage while treatment with the plume transverse to the microchannel showed no cell damage as observed by scanning electron microscopy. Plasma sterilization on hydroxyapatite and dentin surfaces also showed evidence for some line-of-sight mechanism, but in those experiments it could not be ruled out that some hydration effect could have been blocking lower lying cells from being treated. This experiment ruled out the possibility of water blockage since both orientations had the same hydration state. 


\subsection{Future Research}

The information provided in this dissertation provides a guide to future research in the fields of plasma coatings and plasma sterilization. Many questions arose during the research that could not be answered simply and within a short time. The purpose of the dissertation was not to simply report the findings, but also to provide some insight into the underlying mechanisms of each phenomenon such that an optimum solution may be developed.

\subsubsection{Plasma coatings for corrosion and hydrogen re-embrittlement resistance}

Plasma coatings for corrosion and hydrogen re-embrittlement showed great promise as a replacement to chromate conversion coating, but the process remains in its infancy. Other coatings such as electroplated zinc may have a similar performance to chromate coatings, but their use does not signal a dramatic change in the philosophy of corrosion barriers. Therefore, the industry may believe it more cautious to keep with the standard of electroplated passivation coatings although the higher associated costs as compared to coatings produced by vacuum plasma method. Especially in the case of aircraft, where lives and expensive machinery literally hang in the balance, extreme caution must be taken. More experiments must be performed to set at ease the industry's concerns with plasma coating performance and reliability. Plasma coatings are thin as compared to electroplated and vapor deposited metals, therefore coating quality is not so visibly apparent. For instance, ion vapor deposited aluminum is glass bead peened as a standard processing step, but it also serves as a 
quality assurance step to test for coating adhesion. As of now, the quality of plasma coatings has no simple test to ensure performance. Further, plasma coating may be a sensitive process and like any new technology, its modes of failure will at first be unknown. In the experiments thus described, plasma coatings were performed in a continuous process, i.e. the vacuum chamber was evacuated continuously while trimethylsilane was flowed into the chamber at a constant rate. Although tape adhesion testing showed maximum performance using this method, the method may not have been stringent enough for determination of the optimum process parameters. Yu et al. showed plasma coatings produced in a closed reactor system to have better adhesion than with the continuous process due to a change in chemical composition in the coating [1]. Also shown in those experiments were prohesion salt spray tests that outperformed chromate conversion coatings. The industry may have more confidence in coatings produced in the hybrid reactor if these prohesion tests are again performed. These coated parts are also assembled with metal fasteners that may damage coatings upon assembly. The performance of such assemblages needs to be determined before widespread use of plasma coatings is accepted.

\subsubsection{Plasma sterilization}

Rapid sterilization of oral bacteria was achieved, but the mechanisms of sterilization remain unclear. This dissertation presented evidence supporting the theory of a bombarding of charged species as the major cause of sterilization. This is fundamentally different from other researcher's results indicating reactive oxygen species as the major mechanism. It may be the case that the plasma brush used in these experiments was substantially different from other researcher's brushes, primarily: our brush was powered by a direct current power 
source while other researchers tend to use alternating currents in the radio- and micro- wave frequency ranges; our brush was made with argon while other researchers tend to use helium; our brushes geometry was two needle electrodes while other researchers tend to use dielectric barrier discharge type brushes. More research should be performed to elucidate the differences in various plasma sources used in sterilization. Sakiyama et al. has used computer aided numerical simulation of plasma to help explain sterilization results from a radiofrequency produced atmospheric plasma. Simulation of our direct current plasma may also give us insight into the mechanisms of sterilization. Molecular dynamics simulation of the interaction between the plasma and a biological surface such as that of a bacterial cell wall may be too complex at the moment to simulate accurately. An understanding of the temperature and distribution of bombarding species may suffice to determine the likelihood of bombardment as a mechanism of sterilization.

It may be that plasma may be more effective when combined with some other process such as by use of a water jet. Plasma was not found effective in sterilizing lower lying bacteria, so it may not perform well in the treatment of thick biofilms. Water jets have been found to reduce thicknesses of biofilms by $85 \%$, and so pretreatment of biofilms before plasma treatment may be an effective sterilization method [2]. Also, lower lying bacteria may not be killed by the plasma, but the plasma might cause them to be in some state whereby they are more sensitive to antibiotics, such as through disruption of the cell wall. In any case, the efficacy of potential research would be made clearer if the mechanisms of plasma action on the bacteria were better known. To better understand these mechanisms, a number of experiments may be helpful. To determine the effect of reactive oxygen species, destruction of aero-tolerant bacteria should be compared to aero-intolerant bacteria. For instance, this 
dissertation could not completely rule out the possibility of superoxide as a major sterilizing mechanism. E. coli can be cultured in ways to express the soxRS regulon [3]. Similarly, $P$. gingivalis may be cultured to be resistant to $\mathrm{H}_{2} \mathrm{O}_{2}$ [4]. If superoxide or hydrogen peroxide is the major species of sterilization, resistant bacteria may be less affected by plasma treatment. A variety of microorganisms exist as potential sensors for the various species that may be significant. Bacillus atrophaeus also resists sterilization from hydrogen peroxide, Aspergillus niger resists ultraviolet light, Geobacillus stearothermophilus spores resist high heat, radiation, and chemical sterilization [5,6]. Similarly, voltage sensitive dyes may be used during sterilization as a method to measure the voltage developed during plasma treatment [7]. In this manner, a better understanding of the mechanical stresses induced by the electric field and accumulated charge may be obtained.

The efficacy of plasma as a sterilizing tool remains somewhat unclear. It remains unknown what portion of the large variety of bacteria that may be present in the mouth will be killed in such a short amount of time, but the extensive damage compared to some other chemical methods as observed by scanning electron microscopy seems to suggest widespread effectiveness. A comparison between plasma and chemical sterilization rates, as opposed to sterilization modes, needs to be performed on a wide variety of bacteria in order to have a better idea of which method is superior. Although some chemical methods may take longer exposure times, the method of treatment may be more clinically preferred due to the small size of the plume. During caries restoration, there is a difference in opinion whether or not softening of the dentin tends to supersede penetration of bacteria [8]. It is a general consensus that sterilization is not necessary once all mechanically unstable material is removed. As long as a cavity is sealed well, live bacteria do not cause further harm. This may 
altogether dispel the need for sterilizers in the caries restoration process. Therefore, the plasma brush may find better use in caries prevention. At the current stage of development, patients would need to have plasma treatment by a trained clinician due to the high cost of the plasma brush system and dangers inherent. This brings into speculation whether treatment by plasma brush provides a substantial enough benefit over standard brushing, chemical rinses, and/or antibiotics to warrant such treatment. A comparison of the long term effectiveness in vivo still needs to be performed. Also, as stated before, the effectiveness of the plasma brush depends on the geometry of the selected caries. The irregular shape of caries, deep lesions, and pit and fissures present may be out of reach for the plasma used in this dissertation. The plasma brush's ability to rapidly sterilize surfaces may find itself valuable in other fields. I have performed preliminary studies on the sterilization of E. coli on beef. Sterilization was less effective and an undesired desiccating effect was prominent and may again limit its uses.

Sterilization of bacteria through treatment by atmospheric plasma can be extensive and rapid, but many concerns specific to the geometry and hydration state of the seeding medium and bacteria concentration remain. Applicability of the plasma brush should be considered on a case-by-case basis. 


\subsection{References}

1. Yu, Q., Moffitt, C.E., Wieliczka, D.M., Deffeyes, J., Yasuda, H., 2002, “Corrosion protection of ion vapor deposition (IVD) Al-coated Al alloys by low-temperature plasma interface engineering Part III-DC cathodic polymerization in a closed reactor system," Organic Coatings, 44, 37-47.

2. Kato, K., Tamura, K., Nakagaki, H., 2012, "Quantitative evaluation of the oral biofilm-removing capacity of a dental water jet using an electron-probe microanalyzer," Archives of Oral Biology, 57, 30-35.

3. Krapp, A., Humbert, M., Carrillo, N., 2011, "The soxRS response of Escherichia coli can be induced in the absence of oxidative stress and oxygen by modulation of NADPH content," Microbiology, 157, 957-965.

4. Diaz, P., Slakeski, N., Reynolds, E., Renato, M., Rogers, A., Kolenbrander, P., 2006, "Role of oxyR in the oral anaerobe Porphyromonas gingivalis," Journal of Bacteriology, 188, 2454-2462.

5. Keudell A., Awakowicz P., Benedikt J., Raballand V., Yanguas-Gil A., Opretzka C., et al. Inactivation of Bacteria and Biomolecules by Low-Pressure Plasma Discharges. Plasma Processes and Polymers 2010; 7: 327-352.

6. Guizelini, B., Vandenberghe, L., Sella, S., Soccol, C., "Study of the influence of sporulation conditions on heat resistance of Geobacillus stearothermophilus used in the development of biological indicators for steam sterilization," Archives of Microbiology, 194, 991-999.

7. Loew, L., 2011, Membrane Potential Imaging in the Nervous System: Methods and Applications, New York City, NY, USA, Chap. 2.

8. Gardner, A., Higel, R., 1962, "An evaluation of agents used in cavity sterilization," Australian Dental Journal, 7, 53-61. 


\section{VITA}

Adam Blumhagen was born on January 24, 1985 in Portland, OR. In December 2008, he received a Bachelor of Science degree in Mechanical Engineering from the University of Missouri in Columbia, MO. He has been enrolled in the Graduate School at the University of Missouri in the Department of Mechanical and Aerospace Engineering since January 2009. He has been a graduate teacher's assistant in the department since January 2009 and a graduate research assistant in the Surface Science and Plasma Technology Center in University of Missouri since June 2009. 